\title{
mGluR5 modulation as a treatment for Parkinson's disease
}

\author{
Kyle Farmer
}

A thesis submitted to the Faculty of Graduate \& Postdoctoral Affairs

in partial fulfillment of requirements of the degree of

Doctor of Philosophy

in

Neuroscience

Carleton University

Ottawa, ON

Copyright (C) 2018 - Kyle Farmer 


\begin{abstract}
Parkinson's disease is an age related neurodegenerative disease. Current treatments do not reverse the degenerative course; rather they merely manage symptom severity. As such there is an urgent need to develop novel neuroprotective therapeutics. There is an additional need to stimulate and promote inherent neuro-recovery processes. Such processes could maximize the utilization of the existing dopamine neurons, and/or recruit alternate neuronal pathways to promote recovery. This thesis investigates the therapeutic potential of the mGluR5 negative allosteric modulator CTEP in a 6-hydroxydopamine mouse model of Parkinson's disease.
\end{abstract}

We found that CTEP caused a modest reduction in the parkinsonian phenotype after only 1 week of treatment. When administered for 12 weeks, CTEP was able to completely reverse any parkinsonian behaviours and resulted in full dopaminergic striatal terminal re-innervation. Furthermore, restoration of the striatal terminals resulted in normalization of hyperactive neurons in both the striatum and the motor cortex. The beneficial effects within the striatum 
were associated with an increase in activation of mTOR and p70s6K activity. Accordingly, the beneficial effects of CTEP can be blocked if co-administered with the mTOR complex 1 inhibitor, rapamycin. In contrast, CTEP had differential effects in the motor cortex, promoting ERK1/2 and CaMKII $\alpha$ instead of mTOR. Together these data suggest that modulating mGluR5 with CTEP may have clinical significance in treating Parkinson's disease. In addition to the CTEP work, extensive appendices are included in this thesis outlining the optimization of animal models and tools used in the present thesis, as well as other potential therapeutic avenues. 


\section{Manuscripts included in this Thesis}

Farmer, K., Derksen, A., Rowe, E., Thompson, A., Rudyk, C., Prowse, N., Dwyer, Z., Fortin, T., Ferguson, S., \& Hayley, S., (2018). The mGluR5 allosteric modulator CTEP promotes neuro-recovery in the 6OHDA mouse model of Parkinson's disease. In Preparation.

Farmer, K., Rudyk, C., Prowse, N., \& Hayley, S.P., (2015). Hematopoietic cytokines as therapeutic players in early stages Parkinson's disease. Frontiers in Aging Neuroscience.

DOI: $10.3389 /$ fnagi.2015.00126

PMID: 26191001

Farmer, K., Smith, C.A., S.P. Hayley, \& Smith, J.C., (2015). Major alterations of phosphatidylcholine and lysophosphotidylcholine lipids in the substantia nigra using an early stage model of Parkinson's disease. International Journal of Molecular Sciences.

DOI: $10.3390 /$ ijms 160818865

PMID: 26274953 


\section{Other works by Kyle Farmer}

Smith, C.A., Farmer, K., Lee, H., Holahan, M.R., \& Smith, J.C., (2015). Altered Hippocampal Lipid Profile Following Acute Postnatal Exposure to $\operatorname{Di}(2-E t h y l h e x y l)$ Phthalate in Rats. International Journal of Environmental Research \& Public Health.

DOI: $10.3390 /$ ijerph121013542

PMID: 26516880

Barber, A., Farmer, K., Martin, K.R., \& Smith, P.D., (2017). Retinal regeneration mechanisms linked to multiple cancer molecules: A therapeutic conundrum. Progress in Retinal and Eye Research.

DOI: $10.1016 /$ j.preteyeres.2016.08.003

PMID: 27586058

Rudyk, C.A., McNeil, J., Prowse, N., Dwyer, Z., Farmer, K., Litteljohn, D., Caldwell, W., \& Hayley, S., (2017). Age and chronicity of administration dramatically influenced the impact of low dose paraquat exposure on behavior and hypothalamic-pituitary-adrenal activity. Frontiers in Aging Neuroscience.

DOI: 10.3389/fnagi.2017.00222

PMID: 28769783

Litteljon, D., Rudyk, C., Dwyer, Z., Farmer, K., Fortin, T., \& Hayley, S., (2017). The impact of murine LRRK2 G2019S transgene overexpression on acute responses to inflammatory challenge. Brain, Behaviour, and Immunity.

DOI: $10.1016 /$ j.bbi.2017.09.002

PMID: 28893563 


\section{Acknowledgments}

I would like to take the opportunity to thank all the lab members of the Hayley lab. Throughout the years their continued support contributed significantly to the completion of this work. I also wish to include a very special thank you to Teresa Fortin. Without Teresa this thesis would have been significantly delayed. Thank you to my committee for their constructive feedback throughout my PhD. Finally, a special thank you to my supervisor Dr. Shawn Hayley. Shawn has supported me throughout my University education, and I can say without reservation that his supervision has made me into the scientist I am today. 


\section{Dedication}

This thesis is dedicated to my wife, Genevieve Delorme. From $\mathrm{BSc}$ to $\mathrm{PhD}$ Gen has been always there. Without Gen it would have been impossible for me to work the hours I worked, fought the battles I fought, and did the science I did. Gen has kept me happy and healthy, and there is no doubt in my mind that her continued support played a significant role in getting me to where I am now. 


\section{List of Abbreviations \& Definitions}

\begin{tabular}{|c|c|}
\hline 2R,4R-APDC & $\begin{array}{l}\text { (2R,4R)-4-aminopyrrolidine-2,4-dicarboxylate; } \\
\text { Group II mGluR agonist }\end{array}$ \\
\hline 4E-BP1 & $\begin{array}{l}\text { Eukaryotic translation initiation factor 4E-binding } \\
\text { protein 1; translation repressor protein }\end{array}$ \\
\hline 6-OHDA & 6-hydroxydopamine; monoamine neurotoxin \\
\hline $\mathrm{A} / \mathrm{P}$ & Anterior/Posterior \\
\hline ACPT-I & $\begin{array}{l}(1 \mathrm{~S}, 3 \mathrm{R}, 4 \mathrm{~S})-1-\text { Aminocyclopentane-1,3,4- } \\
\text { tricarboxylic acid; selective Group III mGluR } \\
\text { agonist }\end{array}$ \\
\hline AKT & $\begin{array}{l}\text { Protein Kinase K, serine/threonine-specific protein } \\
\text { kinase }\end{array}$ \\
\hline AMPA & $\begin{array}{l}\alpha \text {-amino-3-hydroxy-5-methyl-4-isoxazolepropionic } \\
\text { acid; ionotropic glutamatergic receptor }\end{array}$ \\
\hline ANOVA & Analysis of Variance \\
\hline Arc & $\begin{array}{l}\text { Activity-regulated cytoskeleton-associated protein; } \\
\text { plasticity related protein }\end{array}$ \\
\hline Basimglurant & $\begin{array}{l}\text { 2-chloro-4-(1-(4-fluorophenyl)-2,5-dimethyl-1H- } \\
\text { imidazol-4-ylethynyl)pyridine; Roche selective } \\
\text { mGluR5 negative allosteric modulator }\end{array}$ \\
\hline BBB & Blood Brain Barrier \\
\hline BDNF & Brain Derived Neurotrophic Factor \\
\hline CNS & Central Nervous System \\
\hline CTEP & $\begin{array}{l}\text { (2-chloro-4-((2,5-dimethyl-1-(4- } \\
\text { (trifluoromethoxy)phenyl)-1H-imidazol-4- } \\
\text { yl)ethynyl)pyridine); selective mGluR5 negative } \\
\text { allosteric modulator }\end{array}$ \\
\hline $\mathrm{D} / \mathrm{V}$ & Dorsal/Ventral \\
\hline DA & Dopamine \\
\hline DAG & diacylglycerol; lipid species \\
\hline DAT & Dopamine Transporter \\
\hline DBS & Deep Brain Stimulation \\
\hline
\end{tabular}




\begin{tabular}{ll}
\hline Dipraglurant & $\begin{array}{l}\text { 6-fluoro-2-(4-(pyridin-2-yl)but-3-yn-1- } \\
\text { yl)imidazo(1,2-a)pyridine; Addex selective } \\
\text { mGluR5 negative allosteric modulator }\end{array}$ \\
\hline DOPAC & $\begin{array}{l}\text { 3,4-Dihydroxyphenylacetic acid; metabolite of } \\
\text { Dopamine }\end{array}$ \\
\hline eEF2k & $\begin{array}{l}\text { Eukaryotic elongation factor-2 kinase; protein } \\
\text { kinase }\end{array}$ \\
\hline EGF & Epidermal Growth Factor \\
\hline eIF4A & $\begin{array}{l}\text { eukaryotic initiation factor-4A; cap-dependent } \\
\text { translation initiation factor }\end{array}$ \\
\hline eIF4B & $\begin{array}{l}\text { Eukaryotic translation initiation factor 4B; cap- } \\
\text { dependent translation initiation factor }\end{array}$ \\
\hline EPO & Erythropoietin; cytokine \\
\hline ER & Endoplasmic Reticulum \\
\hline ERK1/2 & Extracellular Signal-Regulated Kinase 1/2 \\
\hline ESI & Electrospray Ionization \\
\hline FGF & Fibroblast Growth Factor \\
\hline FKBP12 & $\begin{array}{l}\text { Peptidyl-prolyl cis-trans isomerase FKBP1A; } \\
\text { immunophilin protein that suppresses signal } \\
\text { transduction }\end{array}$ \\
\hline FosB & $\begin{array}{l}\text { FBJ murine osteosarcoma viral oncogene homolog } \\
\text { B; transcription factor }\end{array}$ \\
\hline FR180204 & ERK1/2 inhibitor \\
\hline FRB & $\begin{array}{l}\text { FKB12-Rapamycin Binding Domain; inhibitory } \\
\text { mTOR binding site for Rapamycin }\end{array}$ \\
\hline GDNF & Glial cell-derived neurotrophic factor \\
\hline GM-CSF & $\begin{array}{l}\text { Granulocyte Macrophage-Colony Stimulating } \\
\text { Factor; cytokine }\end{array}$ \\
\hline GPCR & G-protein Coupled Receptor \\
\hline GTPase & $\begin{array}{l}\text { Hydrolyzing enzymes that target guanosine } \\
\text { triphosphate }\end{array}$ \\
\hline HPLC & High-performance liquid chromatography \\
\hline HVA & Homovanillic acid; metabolite of Dopamine \\
\hline i.p. & intraperitoneal \\
\hline
\end{tabular}




\begin{tabular}{ll}
\hline JNK1/2/3 & c-Jun N-terminal kinases 1/2/3 \\
\hline KN-93 & CaMKII inhibitor \\
\hline KO & Knock Out (genetic) \\
\hline L-AP4 & $\begin{array}{l}\text { L-2-amino-4-phosphonobutyric acid; selective } \\
\text { Group III mGluR agonist }\end{array}$ \\
\hline LC3 I/II & $\begin{array}{l}\text { Microtubule-associated proteins 1A/1B light chain } \\
\text { 3A; effector of autophagy }\end{array}$ \\
\hline L-Dopa & levodopa \\
\hline LID & Levodopa-Induced Dyskinesia \\
\hline LPC & Lysophosphotidylcholines \\
\hline LPS & Lipopolysaccharide; bacterial endotoxin \\
\hline LTD & Long Term Depression \\
\hline LYS379268 & $\begin{array}{l}\text { 2-oxa-4-aminobicyclo(3.1.0)hexane-4,6- } \\
\text { dicarboxylic acid; Group II mGluR agonist }\end{array}$ \\
\hline M/L & Medial/Lateral \\
\hline MAP2K4/7 & mitogen-activated protein kinase kinase 4/7 \\
\hline MAPK & mitogen-activated protein kinase \\
\hline Mavoglurant & $\begin{array}{l}\text { Methyl (3aR,4S,7aR)-4-hydroxy-4-[(3- } \\
\text { methylphenyl)ethynyl]octahydro-1H-indole-1- } \\
\text { carboxylate; Novartis selective mGluR5 negative } \\
\text { allosteric modulator }\end{array}$ \\
\hline MFB & Medium Forebrain Bundle \\
\hline mGluR1-8 & Metabotropic Glutamate Receptors 1-8 \\
\hline MPEP & $\begin{array}{l}\text { 2-Methyl-6-(phenylethynyl)pyridine; selective } \\
\text { mGluR5 negative allosteric modulator }\end{array}$ \\
\hline MPTP & 1-methyl-4-phenyl-1,2,3,6-tetrahydropyridine \\
\hline MTEP & $\begin{array}{l}\text { 3-((2-Methyl-4-thiazolyl)ethynyl)pyridine; } \\
\text { selective mGluR5 negative allosteric modulator }\end{array}$ \\
\hline mTOR & mammalian target of rapamycin; protein kinase \\
\hline mTORC1/2 & mammalian target of rapamycin complex 1/2 \\
\hline NAM & Negative Allosteric Modulator \\
\hline NMDA & $\begin{array}{l}\text { N-Methyl-D-aspartic acid; ionotropic } \\
\text { glutamatergic receptor }\end{array}$ \\
\hline p38 & p38 mitogen-activated protein kinases \\
\hline
\end{tabular}




\begin{tabular}{ll}
\hline p70s6K & $\begin{array}{l}\text { Ribosomal protein S6 kinase beta-1; } \\
\text { serine/threonine protein kinase }\end{array}$ \\
\hline PAM & Positive Allosteric Modulator \\
\hline PC & phosphatidylcholines \\
\hline PD & Parkinson's disease \\
\hline PDCD4 & $\begin{array}{l}\text { Programmed cell death protein 4; initiator of } \\
\text { apoptosis }\end{array}$ \\
\hline PFA & Paraformaldehyde \\
\hline PI3K & $\begin{array}{l}\text { Phosphatidylinositol-4,5-bisphosphate 3-kinase; } \\
\text { intracellular signal transducer enzyme }\end{array}$ \\
\hline PKC 8 & $\begin{array}{l}\text { Protein kinase C delta type; serine/threonine } \\
\text { protein kinase }\end{array}$ \\
\hline PLA2 & Phospholipases A2; lipid enzyme \\
\hline PPAR & $\begin{array}{l}\text { peroxisome proliferator-activated receptor; } \\
\text { transcription factor }\end{array}$ \\
\hline PRAS40 & $\begin{array}{l}\text { Proline-rich AKT1 substrate 1; member of mTOR } \\
\text { complex 1 unit }\end{array}$ \\
\hline Rac & subfamily of the Rho family of GTPases \\
\hline Rheb & $\begin{array}{l}\text { Ras homolog enriched in brain; GTP-binding } \\
\text { protein }\end{array}$ \\
\hline Rho & Rho family of GTPases \\
\hline ROS & Reactive Oxygen Species \\
\hline RPM & Revolutions Per Minute \\
\hline S.c. & sub cutaneous \\
\hline SAM & Silent Allosteric Modulator \\
\hline shRNA & short hairpin ribonucleic acid \\
\hline SNc & Substantia nigra \\
\hline SP & Sphingolipids \\
\hline TH & Tyrosine Hydroxylase \\
\hline TSC1/2 & Tuberous sclerosis 1/2; GTPase-activating protein \\
\hline ULK1 & $\begin{array}{l}\text { Unc-51 like autophagy activating kinase; protein } \\
\text { kinase related to autophagy }\end{array}$ \\
\hline VTA & Ventral tegmental area \\
\hline & \\
\hline
\end{tabular}




\begin{tabular}{ll} 
VU0155041 & $\begin{array}{l}\text { cis-2-[[(3,5- } \\
\text { Dichlorophenyl)amino]carbonyl]cyclohexanecarbo } \\
\text { xylic acid; mGluR4 positive allosteric modulator }\end{array}$ \\
\hline WT & Wild Type (genetic) \\
\hline
\end{tabular}

xiii 


\section{Contents}

mGluR5 modulation as a treatment for Parkinson's disease ............... i

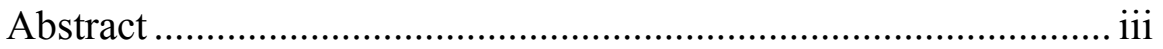

Manuscripts included in this Thesis ............................................. $\mathrm{V}$

Other works by Kyle Farmer ..................................................... vi

Acknowledgments................................................................. vii

Dedication ......................................................................... viii

List of Abbreviations \& Definitions ............................................... ix

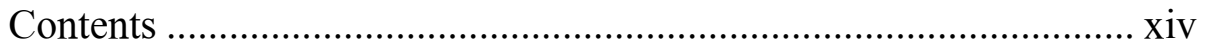

Figures........................................................................... Xvii

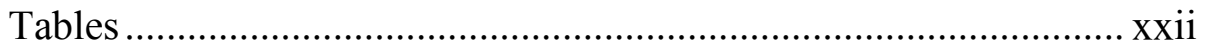

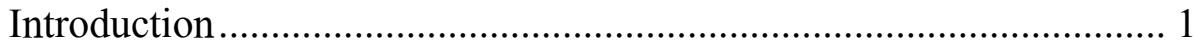

Parkinson's disease …........................................................ 1

Modeling PD in a rodent: 6-OHDA ............................................. 9

Current PD treatment strategies .............................................. 13

The role of glutamate in PD ......................................................... 16

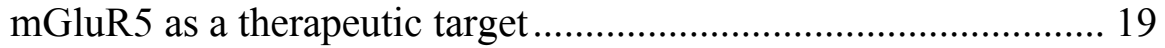

Modulating mGluR5 .................................................................... 24

Experimental prospectus ........................................................... 31

Model Determination ................................................................... 33

xiv 


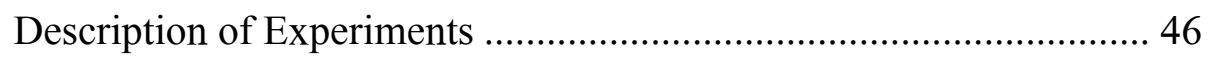

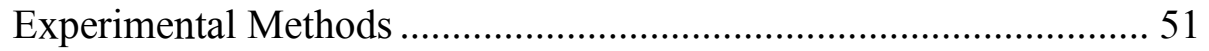

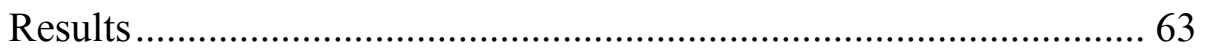

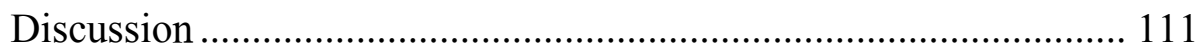

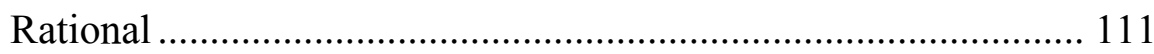

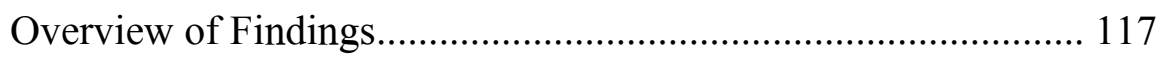

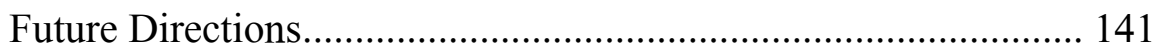

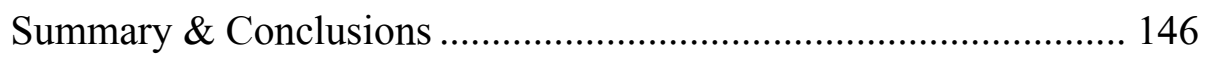

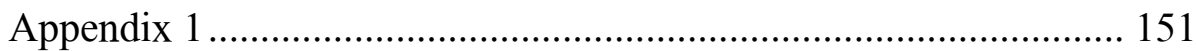

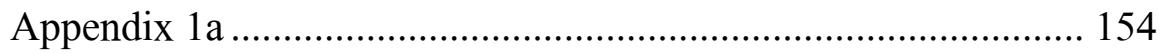

Cytokines as novel trophic and immune modulatory treatments

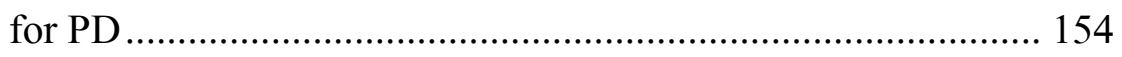

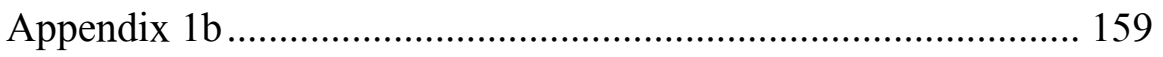

Hematopoietic cytokines as therapeutic players in early stages

Parkinson's disease ................................................................... 159

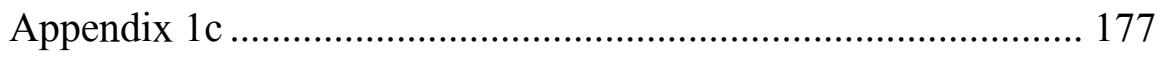

Development of an early stage model of Parkinson's disease

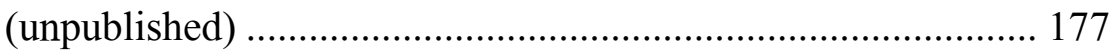

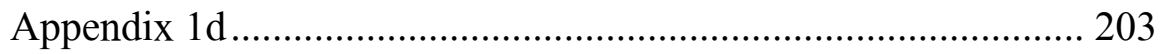

GM-CSF in a late stage model of Parkinson's disease

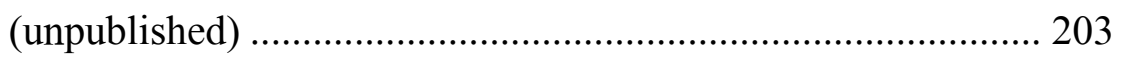

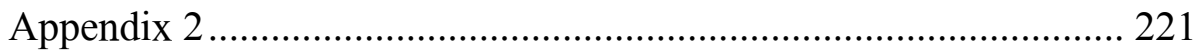


Major alterations of phosphatidylcholine and

lysophosphotidylcholine lipids in the substantia nigra using an

early stage model of Parkinson's disease ............................... 222

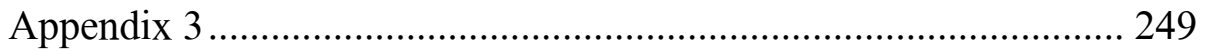

Spontaneous re-innervation in the 6-OHDA model of Parkinson's disease (unpublished) ............................................................ 249

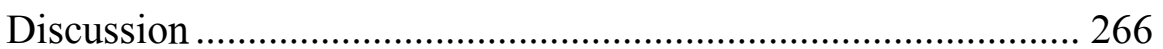

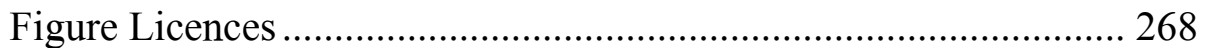

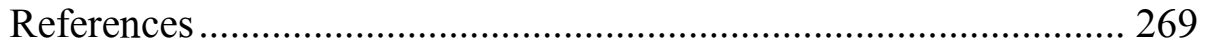




\section{Figures}

Figure 1 Traditional signalling cascade of the Group I mGluR

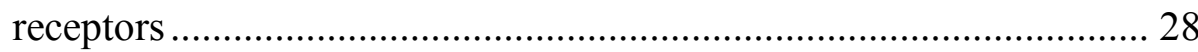

Figure 2 Selective Increase following administration of NAMs ........ 29

Figure 3 Apomorphine rotations (6-OHDA Titration) ......................... 39

Figure 4 Rota Rod (6-OHDA Titration) ............................................ 40

Figure 5 Striatal TH Integrated Density (6-OHDA Titration) ............. 41

Figure 6 SNc cell loss (6-OHDA Titration) ........................................ 42

Figure 7 CTEP Experimental Time-courses ......................................... 61

Figure 8 Apomorphine Induced Rotations (1 week CTEP)................. 65

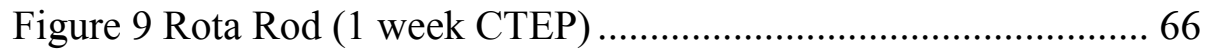

Figure 10 Striatal TH+ Histology (1 week CTEP) ................................ 71

Figure 11 Striatal p-ERK1/2(Tyr204) (1 week CTEP)........................ 72

Figure 12 Striatal p-CaMKII $\alpha($ Thr286) (1 week CTEP) ..................... 73

Figure 13 Striatal p-mTOR(Ser2448) (1 week CTEP) ....................... 74

Figure 14 Striatal p-p70S6K(Thr389) (1 week CTEP)....................... 75 
Figure 15 Striatal BDNF (1 week CTEP) ……………………….... 76

Figure 16 Motor Cortex p-ERK1/2(Tyr204) (1 week CTEP)............. 77

Figure 17 Motor Cortex p-CaMKII $\alpha(\operatorname{Thr} 286)$ (1 week CTEP).......... 78

Figure 18 Motor Cortex p-mTOR(Ser2448) (1 week CTEP) .............. 79

Figure 19 Apomorphine Induced Rotations (12 week CTEP)............ 82

Figure 20 Reversal water maze (12 week CTEP) ................................ 83

Figure 21 Rota Rod (12 week CTEP) .............................................. 84

Figure 22 Striatal TH+ Histology (12 week CTEP) .......................... 87

Figure 23 Nigral TH+ cell counts (12 week CTEP) ......................... 88

Figure 24 Striatal FosB+ cells (12 week CTEP).................................. 89

Figure 25 Motor Cortex FosB + cells (12 week CTEP) ……………... 90

Figure 26 Striatal p-ERK1/2(Tyr204) (12 week CTEP)..................... 93

Figure 27 Striatal p-CaMKII $\alpha($ Thr286) (12 week CTEP) .................. 94

Figure 28 Striatal p-mTOR(Ser2448) (12 week CTEP) ...................... 95

Figure 29 Striatal p-p70S6K(Thr389) (12 week CTEP) ..................... 96

Figure 30 Striatal BDNF (12 week CTEP) ………………………..... 97

Figure 31 Motor Cortex p-mTOR(Ser2448) (12 week CTEP)........... 98 
Figure 32 Motor Cortex p-ERK1/2(Tyr204) (12 week CTEP)........... 99

Figure 33 Motor Cortex p-CaMKII $\alpha(T h r 286)$ (12 week CTEP)...... 100

Figure 34 CTEP Washout Apomorphine Induced Rotations............. 102

Figure 35 CTEP Washout Striatal TH+ Histology ............................ 103

Figure 36 Rapamycin+CTEP Apomorphine Induced Rotations ...... 107

Figure 37 Rapamycin+CTEP Striatal TH+ Histology ...................... 108

Figure 38 Rapamycin+CTEP Striatal BDNF ……............................. 109

Figure 39 Rapamycin+CTEP Striatal p-p70S6K(Thr389) ............... 110

Figure 40 Proposed Mechanism of CTEP action in Striatal DA

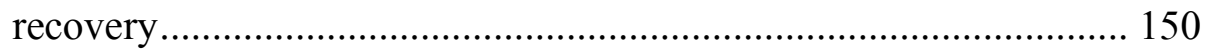

Figure 41 Appendix 1b - Striatal damage. .......................................... 168

Figure 42 Appendix 1b - Stereological count of the SNc ................ 169

Figure 43 Appendix 1b - Stereological count of the SNc at Day 15172

Figure 44 Appendix 1b - Striatal damage at Day 15 ........................ 173

Figure 45 Appendix 1b - Microglial Activation at Day 15 ............... 174

Figure 46 Appendix 1c - Prodromal model time course ................... 185

Figure 47 Appendix 1c - Micromax.................................................. 189

Figure 48 Appendix 1c - Rota Rod ................................................ 190

$$
\text { xix }
$$


Figure 49 Appendix 1c - Apomorphine induced rotations 191

Figure 50 Appendix 1c - HPLC Striatal Dopamine 194

Figure 51 Appendix 1c - HPLC Striatal DOPAC 195

Figure 52 Appendix 1c - HPLC Striatal HVA 196

Figure 53 Appendix 1c - LC3 I:II in the striatum 197

Figure 54 Appendix 1c - Wintergreen Oil Aversion Pilot 202

Figure 55 Appendix 1d - Apomorphine Rotations Pre-Treatment... 214

Figure 56 Appendix 1d - Apomorphine Rotations Post Treatment.. 215

Figure 57 Appendix 1d - Rota Rod .............................................. 216

Figure 58 Appendix 1d - Watermaze ............................................ 217

Figure 59 Appendix 2 - Changes in Phosphatidylcholines .............. 237

Figure 60 Appendix 2 - Changes in lysophosphatidylcholines........ 238

Figure 61 Appendix 3 - Apomorphine Rotations Pre Treatment ..... 260

Figure 62 Appendix 3 - Apomorphine Rotations Post Washout...... 261

Figure 63 Appendix 3 - Rota Rod ................................................... 262

Figure 64 Appendix 3 - Watermaze ................................................. 263

Figure 65 Appendix 3 - Striatal TH Integrated Density.................... 264 
Figure 66 Appendix 3 - SNc cell loss......................................... 265 


\section{Tables}

Table 1 Comparison of mGluR5 NAM pharmacokinetics ................ 30

Table 2 Methods - CTEP Experimental Group Designs .................. 62

Table 3 Appendix 1c - Summary of Catwalk Data ...................... 188

Table 4 Appendix 1d-GM-CSF in a late stage model of PD: Study

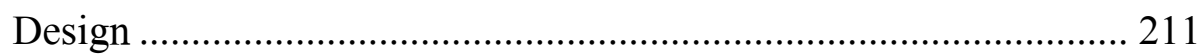

Table 5 Appendix 2 - Summary table of lipidomic findings........... 236 


\section{Introduction}

\section{Parkinson's disease}

Parkinson's disease (PD) is an age related neurodegenerative disease characterized by degeneration of the dopaminergic nigrostriatal pathway and the accumulation of insoluble protein aggregates known as Lewy bodies (Klockgether, 2004; Factor and Weiner, 2008). The nigrostriatal pathway consists primarily of dopamine (DA) neurons within a region of the midbrain known as the substantia nigra that project forward to the basal ganglia, particularly to the globus pallidus and areas of the dorsal striatum including the caudate nucleus and the putamen. These regions are extremely important for the initiation and control of motor movement. Due to the loss of the dopaminergic innervation of these areas PD patients will present with four cardinal symptoms; tremor at rest, rigidity of the distal limbs, bradykinesia (slowing of voluntary movement), and finally gait disturbance and postural instability. In addition to the four cardinal symptoms there are various nonspecific symptoms of PD which include, but are not limited 
to, fatigue, reduced energy, limb stiffness, lack of sensation in the extremities (Factor and Weiner, 2008), depression, as well as olfactory dysfunction (Okun et al., 2009).

Unfortunately, there exist no circulating biomarker that can identify the disease, with the physical symptoms being the only qualifiers capable of effectively diagnosing PD (Miller and O'Callaghan, 2015). Furthermore, patients will only begin to present with hallmark symptomatology once there has been significant loss of the nigrostriatal system, with estimates being that a loss of approximately $50 \%$ of DA neurons of the substantia nigra $(\mathrm{SNc})$ and approximately $80 \%$ of striatal dopamine is required prior to behavioural symptom manifestation (Bernheimer et al., 1973; Toulouse and Sullivan, 2008). As such, what occurs in the initiating and early stages of the disease is not well known. It is now widely accepted that PD is a disease of complex origin that requires multiple insults targeted within the SNc, that individually are not sufficient to induce PD pathology, however when summed produces the hallmark degeneration of dopaminergic neurons and production of insoluble protein inclusions; this hypothesis is commonly known as the multi-hit hypothesis (Carvey 
et al., 2003; Hawkes et al., 2007; Sulzer, 2007). The multi-hit hypothesis frames complex neuropathology such as PD as a disorder resulting from an interactive effect of repeated exposure to various environmental, immune, and genetic stressors.

Identifying environmental contributions can be difficult to confirm as we are often reliant on epidemiological correlations. Furthermore, incomplete information such as frequency of exposures, doses, or native protective resilience factors can often limit the ability to differentiate causative factors from associative factors. Historically there have been many industries associated with an increased risk of developing PD. It is now accepted that it isn't the industry in which you work that increases rates of PD, rather specific toxins that you may be exposed to while at work. Indeed, among agricultural workers (an employment commonly associated with increased prevalence of PD), only those in which crops that require heavy pesticide use do we see an increase risk of developing PD (Moisan et al., 2011). Pesticides are not the only compounds that have been linked to PD. Increased exposure to specific metals, e.g. iron, copper, and manganese, have also been shown to increase ones risk of developing the neurodegenerative disease (Sofic 
et al., 1988; Gorell et al., 1997). In both cases it seems that these toxicants increase overall oxidative stress within the neuron, eventually leading to cell death.

The immune system activation and reactivity is thought to play an important role as a mechanism that might link environmental insults to the development of PD. Persistent inflammation due to continually activated microglia results in chronically elevated levels of cytokines (eg. TNF $\alpha$, IL-1 $\beta$, IL-6, INF $\gamma$, etc.) (Hunot et al., 1996; Hirsch et al., 1998; Nagatsu et al., 2000). These elevated cytokines increase reactive oxygen species (ROS) to toxic levels, eventually initiating pro-apoptotic processes. Indeed, chronically activated immune system and elevated serum cytokines levels is often seen in PD patients (Hunot et al., 1996; Hirsch et al., 1998; Nagatsu et al., 2000). Additionally, there is historical epidemiological evidence indicating exposure to certain early life illness may be correlated with increased incidence of PD. Most famously, in the 1950s the neurologists Pesoskanzer and Schwab reported an overall increase incidence of PD in their patient population and noted that $51 \%$ of their patients were previously infected during the 1918 Spanish Flu epidemic (Schwab et al., 1956; Poskanzer and Schwab, 1961). Similar 
associations have been made with various virus', including intra-uterine influenza (Mattock et al., 1988), and more recently the H5N1 Influenza A, more commonly known as "The Bird Flu” (Jang et al., 2009; Vlajinac et al., 2013). Despite these associations, with increased time and epidemiological data there appears to be no specific virus that causes PD (Estupinan et al., 2013). Rather it is believed that such early life viral exposures may act as the first "hit" in the multi-hit hypothesis. That such a strong immune challenge primes the central nervous system (CNS) and is the first step towards a chronically activated immune system. Indeed, there is a large body of evidence that demonstrates that continual immune challenge, e.g. exposure to bacterial endotoxin lipopolysaccharide (LPS), results in chronically elevated cytokines which can ultimately result in nigral cell loss and parkinsonian phenotypes (Mangano and Hayley, 2009; Mangano et al., 2011; Bobyn et al., 2012; Rudyk et al., 2017).

The vast majority ( $\sim 90 \%)$ of PD cases are of an idiopathic origin. Less than $10 \%$ of patients present with a pathogenic mutation. While relatively rare these mutations provide unique insights into potential disease-causing pathways. Interestingly most of the implicated genes 
can be grouped into one of three potential categories: (1) Autophagy related, (2) Mitochondrial related, or (3) unknown function. For example, parkin (PARK2) acts primarily as an E3 ubiquitin ligase labeling proteins for lysosomal degradation (Thomas and Beal, 2007). Similarly, UCH-L1 (PARK5) maintains ubiquitin binding in cytosolic proteins ensuring their eventual collection for degradation (Osaka et al., 2003). Comparatively, PINK1 (PARK6) acts as a mitochondrial kinase and DJ-1 (PARK7) as a redox sensitive chaperone (Thomas and Beal, 2007). Despite much research, many PD associated genes have no known function (eg. PARK3, PARK4, PARK10, PARK11, and PARK12) (Thomas and Beal, 2007). Indeed, it has been shown that most of these genes play multiple roles within the cell. For example LRRK2, the most prevalent affected gene in $\mathrm{PD}$, has been described to play important roles in autophagy (Manzoni and Lewis, 2017), oxidative stress (Heo et al., 2010), and immunological contributions in PD (Hakimi et al., 2011; Litteljohn et al., 2017).

Unlike other neurodegenerative diseases, such as Alzheimer's disease that sees more global degeneration of neurons, PD results in primarily dopaminergic neuronal loss of the SNc. Interestingly, the 
ventral tegmental area (VTA), a dopamine neuron rich region directly adjacent to the SNc is minimally affected in PD. Thus, dopamine neurons of the SNc are quite unique. This unique vulnerability might stem from an overall higher basal rate of mitochondrial oxidative phosphorylation and a smaller respiratory reserve capacity of $\mathrm{SNc}$ neurons (Consiglia Pacelli, Nicolas Giguère, Marie-Josée Bourque, Martin Lévesque, Ruth S. Slack, 2015). Additionally, SNc DA neurons have increased dendritic arborisation complexity and a higher level of axonal mitochondria compared to DA neurons from other brain regions (Consiglia Pacelli, Nicolas Giguère, Marie-Josée Bourque, Martin Lévesque, Ruth S. Slack, 2015). Together, these neuronal differences result in SNc DA neurons that are functioning at near maximum metabolic capacity basally. Because of this, SNc DA neurons are unable to easily modify their metabolic needs when challenged with an insult, causing them to be more greatly affected by neurotoxic environments. Furthermore, there is increasing evidence that the dopaminergic neurons of the substantia nigra have a role as potential pacemaker cells, never really having periods of inactivity (Guzman et al., 2009). Together this results in a neuronal subpopulation that is continually metabolically taxed, with minimal opportunity for rest and recovery. 
In addition to DA neurodegeneration, PD is associated with the presence of pathological protein aggregates known as Lewy Bodies (Klockgether, 2004; Kim et al., 2014). Lewy bodies have been found to be aggregates of more than 70 different proteins (Wakabayashi et al., 2007), with $\alpha$-synuclein being the most prevalent (Spillantini et al., 1997). Mutations in the $\alpha$-synuclein gene ( $S N C A$ ) has been linked to familial cases of PD (Polymeropoulos et al., 1997; Krüger et al., 1998). While all patients with PD have Lewy bodies (Hughes et al., 1992), not all patients with Lewy bodies have PD (Braak et al., 2003). Indeed, $\alpha$ synuclein aggregates have also been linked to various other disease including, dementia with Lewy bodies, multiple system atrophy, and Alzheimer's disease. The exact function of $\alpha$-synuclein is unknown, however it is believed that the protein plays a role in maintaining cellular structural integrity and regulating the release synaptic vesicles (Kim et al., 2014). More recent work has started to investigate a potential interaction between $\alpha$-synuclein and mitochondrial protein transporters and its effect on overall mitochondrial function (Di Maio et al., 2016). In all cases, synucleinopathy progresses in stages starting in the brainstem, then moving to the midbrain, and then finally to areas in the 
cerebral cortex (Hughes et al., 1992). Additionally, it has been shown that $\alpha$-synuclein aggregates have prion-like protein properties and are able to propagate, transfect, and accumulate, as is typically observed in

prion disease (Kim et al., 2014). Such $\alpha$-synuclein properties might stem from errors in post-translational modification of the protein, including increased serine and tyrosine phosphorylation (Kim et al., 2014). Indeed, the most common SNCA mutation observed in PD patients is a A53T point mutation which results in increase S129 phosphorylation and subsequent aggregation (Kim et al., 2014). Of particular note, most toxin based models of PD (including 6-OHDA) do not produce this essential component of the disease, or do so with low replicability (Tieu, 2011; Blesa et al., 2012; Tazangi et al., 2014; Jagmag et al., 2016).

\section{Modeling PD in a rodent: 6-OHDA}

There are several ways to model PD in animals but unfortunately, there exists no model that encompasses all the hallmarks of PD. As such, it is important to match the research question being asked with an appropriate model. Current animal models of PD can be grossly divided into two main categories; (1) Toxin models, and (2) 
genetic models. Toxin models typically use some sort of chemical which causes extreme oxidative stress resulting in cell death. Most toxin models do not result in Lewy Body formation or any form of $\alpha$ synuclein pathology (Jackson-Lewis et al., 2012). Some exceptions include rotenone when used in rats (Sherer et al., 2003), and occasionally paraquat or maneb when used in mice (Manning-Bog et al., 2002). Importantly, these toxins consistently result in striatal DA loss and nigral neuronal death often in a dose dependent manner (JacksonLewis et al., 2012). Because of this, toxin models are typically used when investigating potential recovery strategies/mechanisms. Genetic models use various tools including knock outs, knock ins, and mutations to modify specific gene functions. Genetic models are often used to identify potential etiological mechanisms for both familial and idiopathic forms of PD. However, genetic models do present with some limitations. In particular, not all genetic models result in hallmark nigral degeneration and often require using a mixed toxin-genetic model to generate typical PD pathology. Also, in many cases animals with genetic modifications require extensive aging before any PD pathology appears. While this may be more clinically relevant, for early pre- 
clinical tests toxin models are often preferred over genetic models as toxin models are often faster to generate and more cost effective.

The 6-hydroxydopamine (6-OHDA) PD toxin model is perhaps the most widely used system that is able to effectively model several aspects of PD (Deumens et al., 2002; Schober, 2004; Blandini et al., 2008; Le et al., 2013). 6-OHDA is a structural analog of both dopamine and norepinephrine and is actively taken in by DA transporter (DAT) resulting in highly selective catecholaminergic neuronal cell death (Ungerstedt, 1968; Breese and Traylor, 1971). Once in the neuron, the toxin auto-oxidizes into various ROS including peroxides and quinones (Schober, 2004). These ROS species then go on to induce mitochondrial dysfunction, specifically in the mitochondrial electron transport chain complex I (Schober, 2004). This mitochondrial disruption results in the production of even more ROS and eventual cell death (Schober, 2004). While it is believed that 6-OHDA induces cell death predominantly through its auto-oxidation in the cytosol (Sachs and Jonsson, 1975; Kumar et al., 1995; Soto-Otero et al., 2002), there is some evidence that 6-OHDA interacts directly with mitochondria altering levels of NADPH oxidase (Rodriguez-Pallares et al., 2007) and driving changes in 
mitochondrial morphology (Galindo et al., 2012) both in the absence of any major increase of cytosolic ROS.

The neurotoxin 6-OHDA is not able to cross the blood brain barrier so it must be administered centrally; the three main targets are the medial forebrain bundle (MFB), the $\mathrm{SNc}$, or the striatum (Ungerstedt, 1968; Blandini et al., 2008). Injections into the MFB and $\mathrm{SNc}$ induce a large degree of dopamine neuronal death causing near complete degeneration of the region in approximately 72 hours (Faull and Laverty, 1969; Lee et al., 1996; Deumens et al., 2002). Alternatively, injections into the striatum induce a prolonged slow level of degeneration which takes more than 30 days to produce its maximum dose dependent lesion size (Sauer and Oertel, 1994; Blandini et al., 2008). Intra-striatal lesions have become increasingly more popular due to this progressive lesion, a key feature of PD (Sauer and Oertel, 1994). Additionally, there is a growing body of evidence indicating that PD may actually begin in the nerve terminals in the striatum and slowly moves backwards inducing progressive cell death in the $\mathrm{SNc}$ (Chu et al., 2012; Tagliaferro and Burke, 2016). Due to its toxic effects high dose bilateral administration of 6-OHDA is rarely used as it often results in 
debilitating effects including excessive seizures, aphagia (inability to swallow), and adipsia (inability to feel thirst). Together these toxin induced effects often result in animal death shortly after the surgery (Ungerstedt, 1971; Bourn et al., 1972). As such, 6-OHDA is typically administered unilaterally. Indeed unilateral administration of 6-OHDA targeting the nigrostriatal pathway can induce both motor and cognitive deficits (Le et al., 2013). Due to the unilaterality of the model, compensatory control by the unaffected hemisphere can cause the animal to appear to show no deficits in certain motor tasks. However, with the administration of DA receptor agonists (e.g. apomorphine) or DA release stimulators (e.g. amphetamines) motor deficits can be observed (Blandini et al., 2008).

\section{Current PD treatment strategies}

Current therapies of PD do not reverse the degenerative course of the disease; rather they merely manage symptom severity. The primary method of treatment has not changed in more than 40 years and involves the use of levodopa (L-DOPA), a dopamine precursor molecule that is capable of crossing the blood brain barrier (Yahr et al., 1969). L- 
DOPA is actively transported into the brain where it is converted into dopamine by L-amino acid decarboxylase (Yahr et al., 1969). The premise behind the therapy is to compensate for the loss of dopamine as a result of the degeneration of nigral neurons by administering its precursor molecule - which once in the brain can be readily converted into DA itself. Additionally, patients are often also given carbidopa - a drug that inhibits decarboxylase activity preventing L-DOPA from being degraded before being able to cross the blood brain barrier (Horstink et al., 2006). Similarly patients are often prescribed monoamine oxidase inhibitors to decrease the degradation of DA in the brain (Horstink et al., 2006). Although initially very effective, the ability of L-DOPA to manage symptom severity decreases dramatically with prolonged use and disease progression, often increasing the severity of muscle dystonia and dyskinesias (Parkinson Study Group, 2000; Fahn et al., 2004). As patients become less responsive to L-DOPA treatments many become candidates for deep brain stimulation (DBS). The procedure is extremely invasive and requires the implantation of a microelectrode into the subthalamic nucleus or the globus pallidus (Breit et al., 2004); other major regions involved in the cortico-basal ganglia-thalamocortical motor circuit. As is the case with L-DOPA, 
with time DBS treatment become ineffective since the treatment does not stop the progression of the disease.

Despite continual effort, there has been very few therapeutics that have been successfully translated from the laboratory to the clinic. Importantly, in many pre-clinical studies the efficacy of a potential therapeutic is assessed in a manner that mostly tests the compounds ability to prevent PD pathology - frequently researchers report that they start therapeutic administration prior to toxin administration. Unfortunately, this is not the case in the human condition - clinicians simply aren't able to start therapeutics prior to disease progression. This is a major limitation in current literature. The argument can be made that in pre-clinical models, administering a potential therapeutic after PDlike pathology has occurred may result in more neuroprotective therapeutics being successfully translated to the clinic. 


\section{The role of glutamate in PD}

In current $\mathrm{PD}$ research there is a predominant focus on the dopaminergic system due to the very clear effect of the disease on these neurons. Because of this, the role of the glutamatergic neurotransmitter system is often overlooked. Glutamate is the primary excitatory neurotransmitter in the brain with receptors found on approximately 40\% of all synapses (Fonnum, 1984; Greenamyre, 1986; Greenamyre and Porter, 1994). Indeed there are glutamate neurons present in the SNc (Yamaguchi et al., 2013), the striatum, and the motor cortices (Chuhma et al., 2014). Furthermore it has been found that there is increased glutamatergic activity in all of these regions in patients diagnosed with PD (Blandini et al., 1996). Importantly, there is a growing body of literature that shows that the glutamatergic neuronal system plays an important role in the hypothesized etiology of PD (Greenamyre, 1986; Blandini et al., 1996). Similar to dopamine, glutamate is also an extremely oxidative molecule. Under normal physiological conditions glutamate can be enzymatically degraded in a manner that minimizes oxidative stress. However, during conditions of high metabolic demand glutamate is often improperly metabolized, resulting in the generation 
of excessive ROS (Greenamyre, 1986; Blandini et al., 1996). Indeed, this has been reported in SNc neurons basally and even more so during PD progression. In addition to the oxidative stress as a result of the autooxidation of glutamate, there is additional stress induced by increased glutamatergic activation of calcium and sodium ion channels. The inability to buffer large changes in calcium fluxes increases overall cell stress and is believed to exacerbate the neurodegenerative microenvironment and to promote the progression PD pathology (Greenamyre, 1986; Blandini et al., 1996). As such, targeting the glutamatergic system may be an important avenue in treating the neurodegenerative disease (Johnson et al., 2009). Indeed, targeting glutamatergic NMDA (Klockgether et al., 1996; Steece-Collier et al., 2000; Kelsey et al., 2004) and AMPA (Löschmann et al., 1991; Wachtel et al., 1992) receptors systems have demonstrated beneficial effects in PD. In many cases, pharmacological modulation of the glutamatergic system reduced hyper excitability and calcium induced toxicity. Furthermore, there is evidence indicating that modulation of glutamatergic systems might even stimulate neuroplasticity related pathways (Jayakar and Dikshit, 2004; Johnson et al., 2009; Monaghan et al., 2012). Interestingly, one of the largest advances in recent PD 
therapeutics is from the NMDA antagonist Amantadine, which in a recent phase 3 clinical trial was shown to effectively treat L-DOPA induced dyskinesias (Elmer et al., 2018).

While targeting the ionotropic NMDA and AMPA receptors have proven beneficial in alleviating immediate hyperactivity and glutamatergic induced toxicities, they appear to have limited potential for long term recovery. Current research has started focusing on the other major class of glutamate receptors; namely the metabotropic Gprotein coupled mGluRs. To date 8 different mGluRs have been identified and are subcategorized into Group I (mGluR1, 5), Group II (mGlur2, 3), and Group III (mGluR4, 6, 7, 8) based on their homology and ligand selectivity. Nearly all mGluRs have at one point or another been implicated in PD. mGluR1 receptors can be found extensively throughout the basal ganglia, however have limited expression in striatal regions (Testa et al., 1994, 1995; Kerner et al., 1997). Despite its low striatal expression, large changes to mGluR1 expression have been shown in models of PD (Yamasaki et al., 2016). Furthermore selective antagonism of mGluR1 has shown to prevent dopamine depletion following 6-OHDA lesion (Vernon et al., 2007). Despite this 
neuroprotective effect, modulation of mGluR1 has shown limited ability in treating PD motor symptoms (Rylander et al., 2009). The group II mGluRs have also shown promising pre-clinical benefits in PD, resulting in reduced glutamatergic toxicity (Corti et al., 2007) and may also attenuate neurodegeneration by reducing microglial activity and neuro-inflammation (Chan et al., 2010). Similarly, due to their widespread expression throughout the basal ganglia there is a high probability that group III mGluRs are involved in some capacity in PD, however their exact role is currently unknown (Ribeiro et al., 2017). Despite having an unknown etiological role, modulation of the Group III mGluR4 receptor has proven beneficial in reducing behavioural deficits in various animal models of PD (Charvin, 2018).

\section{mGluR5 as a therapeutic target}

Of particular interest for the present thesis is the mGluR5 receptor system as a potential therapeutic target for PD. mGluR5s are expressed through the CNS and can be found predominantly on the postsynaptic terminal in both neurons and astrocytes. Importantly, mGluR5 is highly expressed in the striatum (unlike its Group I 
counterpart, mGluR1), making it an attractive therapeutic target. Stimulation of the mGluR5 receptor results in activation of two main cascades. One pathway is via $\mathrm{G}_{\mathrm{q}^{\prime / 11}}$ signaling resulting in the release of endoplasmic reticulum calcium stores which results in increased CaMKII and ERK1/2 activity. Alternatively, mGluR5 can interact with Homer scaffolds which then (A) modulate NMDA receptor activity, (B) increase ERK1/2 activity, or (C) drive $\mathrm{PI} 3 \mathrm{~K} / \mathrm{AKT} / \mathrm{mTOR}$ signaling (Figure 1). Indeed it has been shown that mGluR5 plays a critical role in regulating glutamatergic systems hyper excitability and that mGluR5 can modulate this hyper excitability in both midbrain and forebrain circuits by altering NMDA receptor currents and regulating intracellular calcium stores (O’Brien, 2004; Rodriguez, 2005; Chen et al., 2007).

mGluR5 receptors have been implicated in various neurodegenerative diseases including Huntington's diseases (Abdelrahman et al., 2017), Alzheimer's disease (Hamilton et al., 2016), and Parkinson's disease (Ambrosi et al., 2010). mGluR5 dysfunction has been specifically implicated in PD synucleinopathy (Price et al., 2010), dyskinesia (Bezard et al., 2014), striatal-cortical hyper excitability (Johnson et al., 2009), and general motor disturbances (Guimaraes et al., 
2015). Targeting mGluR5 in PD is quite attractive due to the fact that it acts as a regulator of both endoplasmic reticulum (ER) calcium homeostasis and NMDA activity. Additionally, the mGluR5 primary signaling partners, ERK1/2 and mTOR, have well documented trophic actions and are known to modulate neuronal plasticity (Binder and Scharfman, 2004; Roskoski, 2012; Maiese, 2015).

ERK1/2 (extracellular signal-regulated kinase) proteins play important roles in regulating cell growth and survival. While technically two individual proteins, all known activators of ERK1/2 cause parallel stimulation of both ERK1 and ERK2 (Lefloch et al., 2009). ERK1/2 work together towards stimulating cellular proliferation, however act in different capacities. ERK1 predominantly drives protein transcription, cell cycle progression, and cell survival, while ERK2 stimulates migration, differentiation, and cytoskeletal remodeling (Roskoski, 2012). It has been shown that rapid activation of ERK proteins is protective in the 6-OHDA toxin model of PD (Lin et al., 2008) and might play a role in mGluR mediated synaptic plasticity (Thomas and Huganir, 2004; Moult et al., 2008). As well, activation of intracellular 
mGluR5 in particular results in ERK1/2 mediated increases of Arc another important mediator of synaptic plasticity (Kumar et al., 2012).

The mammalian target of rapamycin (mTOR) is a kinase protein that forms two different complexes; complex 1 (mTORC1) and complex 2 (mTORC2). Complex 2 acts predominantly as an activator of Rho/Rac GTPases (Li et al., 2004) and PKC $\alpha$ (Ikenoue et al., 2008) - two important mediators of cytoskeletal re-organization. mTORC2 is also known to stimulate the activation of mTORC1, by phosphorylation of AKT (Huang and Manning, 2009). mTORC1 has two main roles - acting as a regulator of autophagy via ULK1, or as a regulator of protein translation and cell cycle processes through 4E-BP1 and p70s6K (Fingar and Blenis, 2004). Through these primary substrates, mTORC1 plays and important role regulating mitochondrial metabolism (Morita et al., 2013), autophagy (Jung et al., 2010), microtubule organization (Jiang and Yeung, 2006), cap-dependent protein translation (Roux and Topisirovic, 2012), ribosome biogenesis (Mayer and Grummt, 2006), lipid synthesis, and lipid metabolism (Laplante and Sabatini, 2009), among other known processes. 
Cytosolic initiators of mTOR activity include elevated glucose levels, hypoxic conditions, genetic and energetic stressors, as well as general neuro-inflammation (Hay and Sonenberg, 2004). Additionally, stimulation of many transmembrane receptors can result in eventual downstream modulation of mTOR activity (Hay and Sonenberg, 2004). Focusing specifically on mGluR5, the main systems of interest include ERK1/2 (Osterweil et al., 2010) and PI3K (Hou and Klann, 2004) which can both lead to activation of mTORC1 (Ghosh et al., 2006). ERK1/2 has also be shown to indirectly activate mTORC1 via phosphorylation of raptor (Carriere et al., 2011). Alternatively, mGluR5 can stimulate the canonical PI3K/AKT signalling cascade which activates 4E-BP1 and p70s6K (Wang et al., 2012).

There is evidence that dysregulation of mTOR plays important causal roles in both idiopathic and familial PD (Lan et al., 2016). The pathogenic role of mTOR in PD appears to be predominantly through its role as a regulator of autophagy. mTORC1 inhibits ULK1 through phosphorylation preventing the stimulation of Beclin, thereby impairing normal autophagic processes (Fingar and Blenis, 2004). However, in PD we find that mTORC1 levels are often significantly decreased resulting 
in dysregulated increases in autophagy. Importantly, decreases in mTOR also diminish 4E-BP1 and p70s6K activity, thereby preventing mTORC1 dependent protein translation and cellular repair processes (Fingar and Blenis, 2004). These two proteins have been shown to regulate the production of various trophic factors, such as brain derived neurotrophic factor (BDNF), epidermal growth factor (EGF), fibroblast growth factor (FGF), and erythropoietin (EPO) (Maiese, 2016). In some cases, such as with BDNF, these trophic factors can then stimulate further mTOR activity in a positive feedback loop (Hay and Sonenberg, 2004). Finally, there is indeed evidence that viral induced expression of upstream activators and downstream effectors of mTOR improve behavioural phenotype and neuropathological outcomes in response to 6-OHDA induced nigral cell death (Kim et al., 2009b, 2011, 2012; Cheng et al., 2011).

\section{Modulating mGluR5}

Selective pharmacological modulation of a $\mathrm{G}$ protein coupled receptor is extremely challenging, due mostly to the homogeneity of the orthostatic binding site found in the various subtypes in receptor 
families. In recent years, advances in protein tomography have allowed for the identification of subtype specific allosteric binding sites that are distinct from the traditional orthostatic binding location. Using designer molecules, it is now possible to selectively target these specific receptor sites. These pharmacological compounds can be classified as (A) positive (PAM), (B) negative (NAM), or (C) silent allosteric modulators (SAM). Positive allosteric modulators typically enhance receptor function, negative allosteric modulators reduce receptor function, while silent allosteric modulators do not modulate orthostatic ligand properties. Importantly, allosteric modulators do not work in traditional agonist/antagonist fashions, rather they simply modulate receptor sensitivity. For example, using a NAM does not completely block receptor activity like a typical antagonist, rather it simply reduces overall receptor sensitivity to stimulatory ligands. Importantly, this can result in differential activation of downstream targets. Further, the activity of some downstream targets may actually increase whereas others may be reduced (please see Figure 2 for pictorial description).

This thesis will assess the potential beneficial effects of treating PD using CTEP (2-chloro-4-((2,5-dimethyl-1-(4- 
(trifluoromethoxy)phenyl)-1H-imidazol-4-yl)ethynyl)pyridine) a potent negative allosteric modulator with very high selectivity to mGluR5 (Lindemann et al., 2011). CTEP binds to the heptahelical domain of the mGluR5 receptor. It binds to mGluR5 with $>1000$ fold selectivity compared to over 100 other targets, including all known mGluRs (Lindemann et al., 2011). While it does not directly interact with the orthostatic ligand site, CTEP has been shown to act as an inverse agonist in a non-competitive manner under basal conditions in cell culture (Lindemann et al., 2011). CTEP is orally bioavailable and can efficiently cross the blood brain barrier (Lindemann et al., 2011; Michalon et al., 2012). Furthermore, CTEP persists in the brain for greater than $48 \mathrm{hrs}$, maintaining a receptor occupancy of approximately $75 \%$ in striatal and cortical regions (Lindemann et al., 2011). Other mGluR5 NAMs do exist, with the main competing compounds being fenobam, MPEP, and MTEP. All three of these compounds have previously been shown to be beneficial at treating L-DOPA induced dyskinesias (Johnston et al., 2010; Rylander et al., 2010; Morin et al., 2016). Furthermore MPEP and MTEP demonstrated modest improvements in behavioural deficits using a 6-OHDA rat model of PD (Spooren et al., 2000; Dekundy et al., 2006). However compared to 
fenobam, MPEP, and MTEP, CTEP binds more readily to mGluR5, has a higher brain penetrance, and persists in the brain more than ten times longer (see Table 1 for a comparison of the pharmacokinetic properties of various mGluR5 NAMs) (Lindemann et al., 2011). To date CTEP has been shown to provoke marked improvements in various conditions, including Fragile X (Lindemann et al., 2011), Alzheimer's disease (Hamilton et al., 2016), and most recently Huntington's disease (Abdelrahman et al., 2017). In each of these cases, CTEP induced marked behavioural improvement, coupled with reduced pathological aggregates and diminished neuronal loss. Ultimately, the properties of CTEP compared to other mGluR5 NAMs makes it an attractive candidate for future clinical translation in PD. 


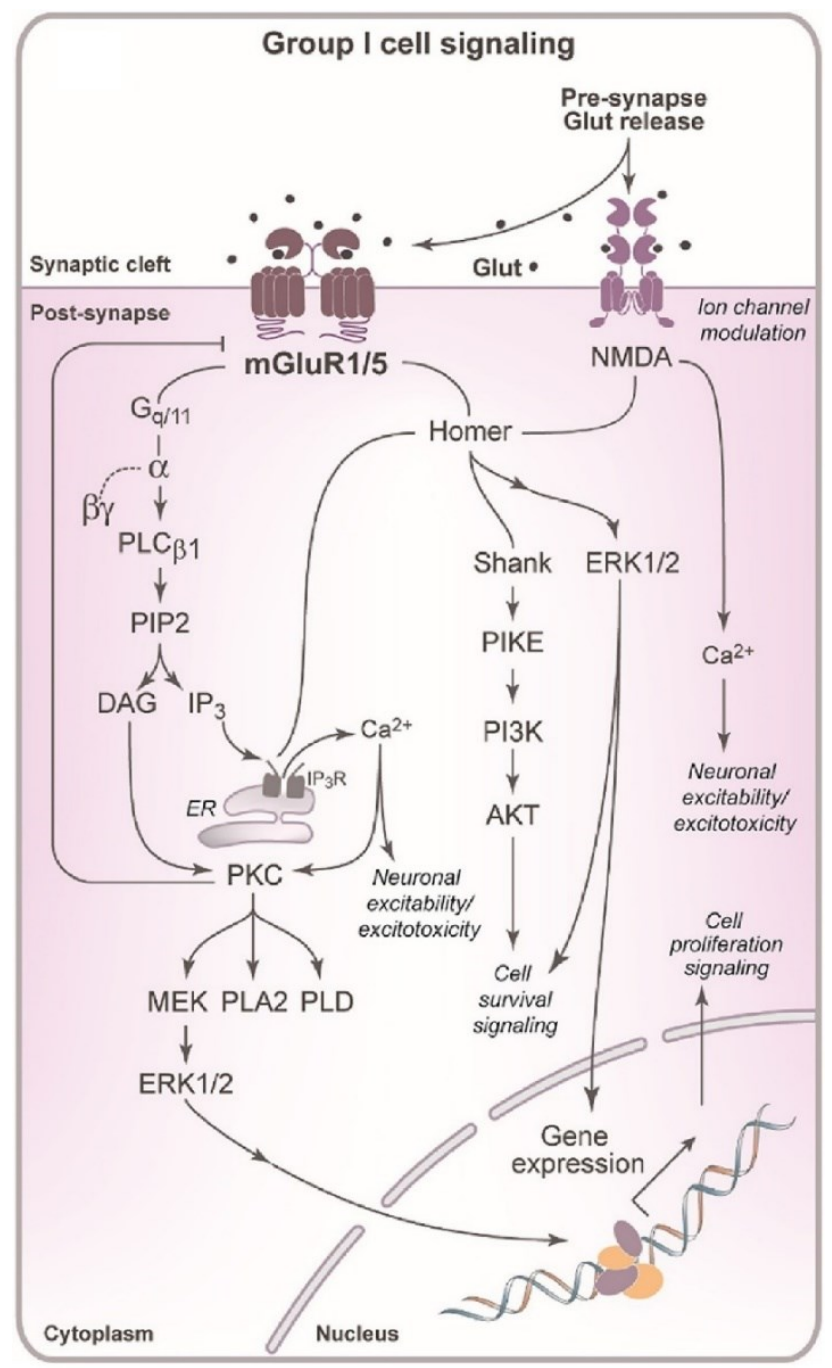

Figure 1 Traditional signalling cascade of the Group I mGluR receptors

Activation of the receptor traditionally causes downstream effects including (A) release of ER calcium stores through $\mathrm{G}_{q^{/ 111}}$ signaling, (B) activation of ERK1/2 through both $\mathrm{G}_{\mathrm{q} / 11}$ and Homer scaffolding, (C) modulation of NMDA function, and (D) activation of PI3K/AKT signalling cascades, including the downstream effector mTOR. Figure modified from (Ribeiro et al., 2017), used with permission from the publisher. 


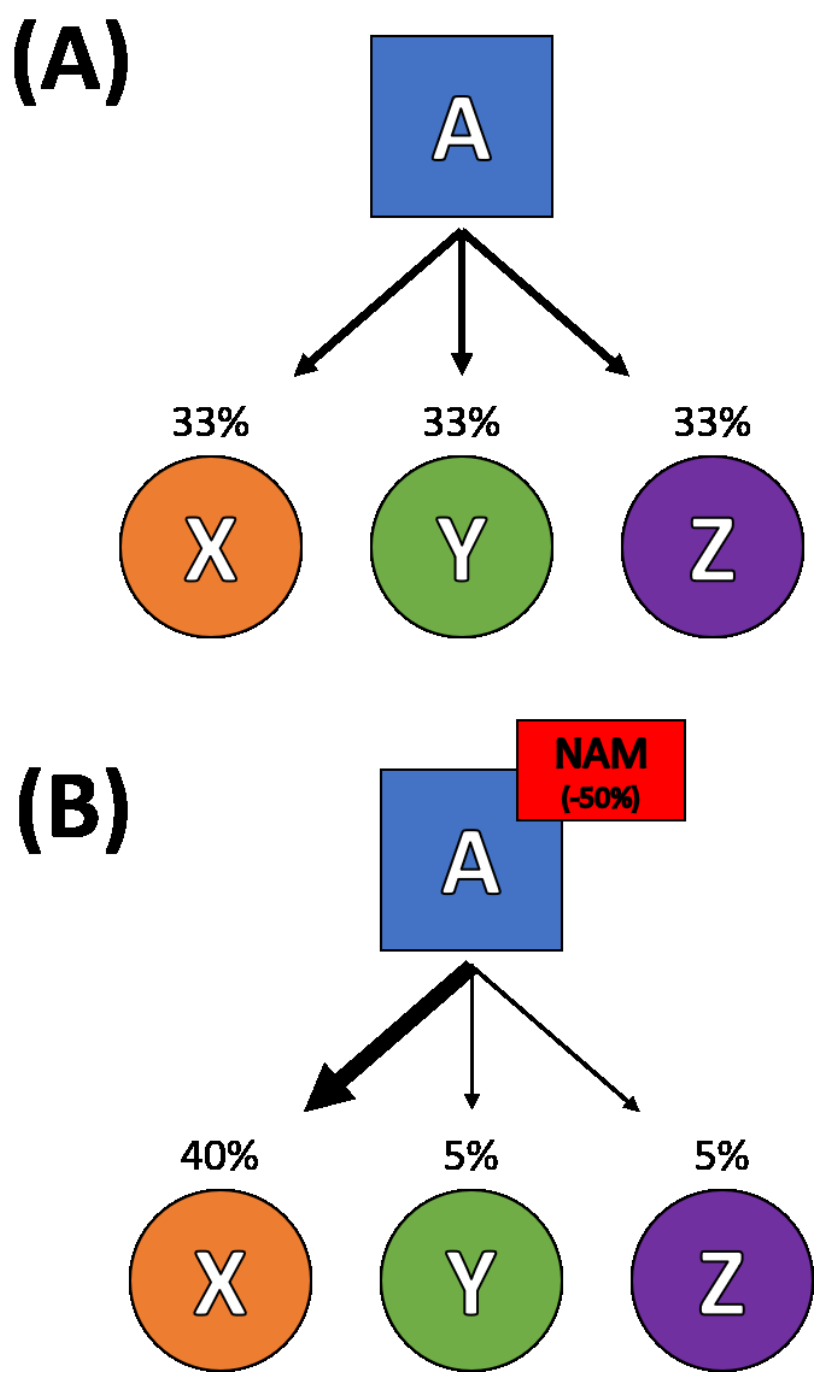

Figure 2 Selective Increase following administration of NAMs

(A) Hypothetical signaling cascade of receptor A which targets each effector equally. (B) Following the application of a NAM which results in an overall decrease in receptor function of $50 \%$, individual target responses may not be equally affected. Indeed in this example, target $X$ has an overall increase in activity compared to panel A, while targets $\mathrm{Y}$ and $\mathrm{Z}$ are greatly reduced. 


\section{Table 1 Comparison of mGluR5 NAM pharmacokinetics}

\begin{tabular}{|c|c|c|c|c|c|}
\hline & Fenobam & MPEP & MTEP & Basimglurant & CTEP \\
\hline \multicolumn{6}{|l|}{ Structure } \\
\hline Species tested & Mouse & Mouse & Mouse & Rat & Mouse \\
\hline Oral Bioavailability (\%) & 7 & 6 & 87 & 42 & 100 \\
\hline $\begin{array}{l}\text { Peak Plasma Concentration } \\
\text { (p.o., ng/mL) }\end{array}$ & 796 & 912 & 7435 & 240 & 716 \\
\hline Half-life (h) & 0.7 & $\mathrm{~N} / \mathrm{A}^{\mathrm{a}}$ & 0.5 & 7.5 & 55 \\
\hline Clearance (ml/min/kg) & 33 & 206 & 82 & 6.0 & 2.1 \\
\hline $\begin{array}{c}\text { Microsomal Clearance } \\
(\mu 1 / \min / \text { ng protein at } 1 \mu \mathrm{M})\end{array}$ & $442.0 \pm 5.7$ & $424.0 \pm 86.9$ & $401.5 \pm 33.2$ & 10.9 & $4.5 \pm 21$ \\
\hline
\end{tabular}

a Intravenous half life is 1.9

* on all metrics, with the exception of peak plasma concentration, CTEP performs better than other comparable mGluR5 NAMs.

Data sourced from (Lindemann et al., 2011, 2015) 


\section{Experimental prospectus}

The currently available PD therapies merely manage symptom severity and are not able stop or even slow the neurodegenerative course of the disease. With this in mind, there is a need to focus on stabilization of the surviving neurons, and most importantly, to promote neurorecovery processes that capitalize upon the inherent neuroplasticity of the basal ganglia. Such processes could maximize the utilization of the existing DA neurons, and/or recruit alternate neuronal pathways to promote compensation. This thesis explores potential avenues in the treatment of PD that attempts to address these core features that are lacking in current treatment strategies. The main body of this thesis explores the use of the metabotropic glutamatergic receptor mGluR5 negative allosteric modulator, CTEP, as such a potential therapeutic.

Importantly, in all cases CTEP is administered after some degree of neurodegeneration has already occurred, thereby making our model more clinically relevant. The various appendices will cover additional areas of PD research including (A) animal model validation and 
limitations, (B) alternative experimental therapeutic avenues, (C) and potential diagnostic methods. 


\section{Model Determination}

Although 6-OHDA is often used in a rats, there still are plenty of studies demonstrating the efficacy of 6-OHDA in mice, with some using intra-striatal injections going back to the 1980s (eg. (Brundin et al., 1986)). However, in many of these early papers there was large variability in the dosing and injection coordinates where the toxin was administered, and even strain of mice used (Bensadoun et al., 1998; Cunningham and Su, 2002; Nishimura et al., 2003; Iwata et al., 2004; Lundblad et al., 2004). As such, we wished to confirm the model by completing a full 6-OHDA dose titration in our CD-1 mice using the anatomical correlates of the unilateral single injection rat coordinates developed by Blandini (2007).

\section{Methods}

\section{$\underline{\text { Animals }}$}

Male CD-1 mice sourced from Charles River (Kingston, Ontario, Canada), aged 6-8 weeks upon delivery were used for all described 
experiments. Animals were individually housed in techniplast blue line individually ventilated polypropylene cages (approx. dimensions: 400x200x200mm) with no enrichment other than nestlets. Animals were maintained on a standard $12 \mathrm{~h}$ light/dark cycle. Tap water and chow (Teklad, \#2014) was provided ad libitum, while room temperature and humidity were maintained at $20^{\circ} \mathrm{C}$ and $50 \%$, respectively. All aspects of this experiment were approved by the Carleton University Committee for Animal Care and adhered to the guidelines outlined by the Canadian Council for the Use and Care of Animals in Research.

\section{Surgery}

The mice were anaesthetized using variable flow isoflurane inhalational anesthetic (1.5-5\% in pure O2). Animals were then placed in a Kopf instruments Model 940 stereotaxic frame (Kopf Instruments, Tujunga, CA, USA). An L-shaped PlasticsOne 3280 cut $3 \mathrm{~mm}$ below the pedestal was inserted into the right striatum at the coordinates $1.00 \mathrm{~mm}$ anterior, $1.75 \mathrm{~mm}$ lateral, and 3.00 ventral relative to bregma and skull surface. A single $2 \mu 1$ infusion of vehicle solution (in $0.9 \%$ saline solution containing $0.02 \%$ ascorbic acid) or 6-hydroxydopamine hydrochloride $(4,8,12,16$, or $20 \mu \mathrm{g}$, Sigma-Aldrich, H4381) was administered at a rate of $0.4 \mu 1 / \mathrm{min}$ using a Harvard apparatus picoplus 
pump equipped with a $10 \mu 1$ Hamilton syringe. The cannula was retracted after a 5 minute incubation period. Bonewax (Stoelting, Wood Dale, IL, USA) was placed over the drill hole and the incision closed using non-absorbable nylon sutures. Animals were given tramadol (20mg/kg at $2.5 \mathrm{mg} / \mathrm{ml}$, Chiron Compounding Pharmacy, Guelph, ON, Canada) twice a day for 3 days, beginning on the day of surgery. Mice were given a 30 day period before any pharmacological manipulation to ensure a maximal 6-OHDA induced lesion.

\section{Behavioural testing}

Prior to each behavioural test, mice were habituated to the testing room for at least a 20 minute period.

\section{Apomorphine Induced rotations}

Animals were administered apomorphine hydrochloride hemihydrate (sub cutaneous injection, $0.5 \mathrm{mg} / \mathrm{kg}$ at $0.1 \mathrm{mg} / \mathrm{ml}$ in $0.9 \%$ saline, SigmaAldrich, A4393) 30 days following the surgical procedure. Briefly, immediately following the injection of apomorphine animals were placed into large open container $(27 \times 48 \times 20 \mathrm{~cm})$ and recorded from above. After a 5 minute drug incubation period, net contralateral rotations were counted over the next 20 minute period. 


\section{Accelerating Rota Rod}

Animals were trained on the rota rod (Omnitech Electronics) over a 2 day period. On day one animals were placed on the rota rod apparatus which was maintained at a continual speed of 11 RPM. If the animal fell from the apparatus they were immediately returned over a 5 minute period. This process was repeated three times total with an inter-trial interval of 60 minutes. Day 2 was a repeat of Day 1, however the rota rod was maintained at speed of 22RPM. On the third day animals were tested using an accelerating protocol with a rotation speed increasing from 4 RPM to 88 RPM over a 600 second period. Maximum speed achieved before falling off the apparatus was recorded. Animals were tested over 3 trials. The lowest of the 3 trials was discarded and the average of the remaining 2 trials were used as the animals score. The test day coincided with the last day of the experiment. To reduce testretest confounds, animals were only tested on the rota rod on the last day of experimentation.

\section{Euthanization}

Animals were deeply anesthetized with an overdose of sodium pentobarbital. Mice were then transcardially perfused using chilled 
$0.9 \%$ saline followed by $4 \%$ paraformaldehyde (PFA) solution in $0.1 \mathrm{M}$ phosphate buffer. Extracted brains were post fixed for $24 \mathrm{hrs}$ in $4 \%$ PFA, followed by sequential washes of $10 \%$ sucrose in $0.1 \mathrm{M}$ phosphate buffer over another $24 \mathrm{hr}$ period. Tissue was then incubated in a $30 \%$ sucrose solution in $0.1 \mathrm{M}$ phosphate buffer for $72 \mathrm{hrs}$. Following this last sucrose wash tissue was flash frozen and stored at $-80^{\circ} \mathrm{C}$.

\section{Histology}

Tissue was sectioned at $40 \mu \mathrm{m}$ on a rotary cryostat. Free floating tissue sections were immuno-labelled overnight in primary antibodies (mouse anti-Tyrosine Hydroxylase (TH) 1:2000 [Immunostar 22941]). Tissue sections were incubated in biotinylated secondary and tertiary streptavidin HRP solutions for $2 \mathrm{hrs}$ each. Using ImageJ software at the site of injection and the two adjoining sites, integrated densities were measured as a ratio to its ipsilateral hemisphere signals after background subtraction. $\mathrm{TH}+$ cells amounts of the $\mathrm{SNc}$ were assessed in a qualitative manner by a blinded experimenter $(0=$ no noticeable cell loss, $1=$ minor cell loss, 2 = approx. $50 \%$ cell loss, $3=$ extensive cell loss). 


\section{Findings}

Thirty days following the injections of 6-OHDA animals were challenged with apomorphine. A one-way ANOVA found a significant dose effect $(F(5,23)=3.85, \mathrm{p}<.05$, and post hoc analysis found that $20 \mu \mathrm{g}$ of 6-OHDA resulted in significantly more net contralateral rotations compared to vehicle controls $(\mathrm{p}<.05)$ (Figure 3$)$. Similarly, compared to controls the $20 \mu \mathrm{g}$ of 6-OHDA also resulted in a significant reduction in the animals ability to remain on the accelerating rota rod $(\mathrm{F}(5,20)=2.74, \mathrm{p}<.05$, post hoc $\mathrm{p}<.05)($ Figure 4$)$, in reduced striatal $\mathrm{TH}+$ fibre integrated density $(\mathrm{F}(5,22)=5.29, \mathrm{p}<.01$, post hoc $\mathrm{p}<.0001)$

(Figure 5), and in reduced nigral TH+ cells $(\mathrm{F}(5,24)=9.98, \mathrm{p}<.0001$, post hoc $<.0001)($ Figure 6). 


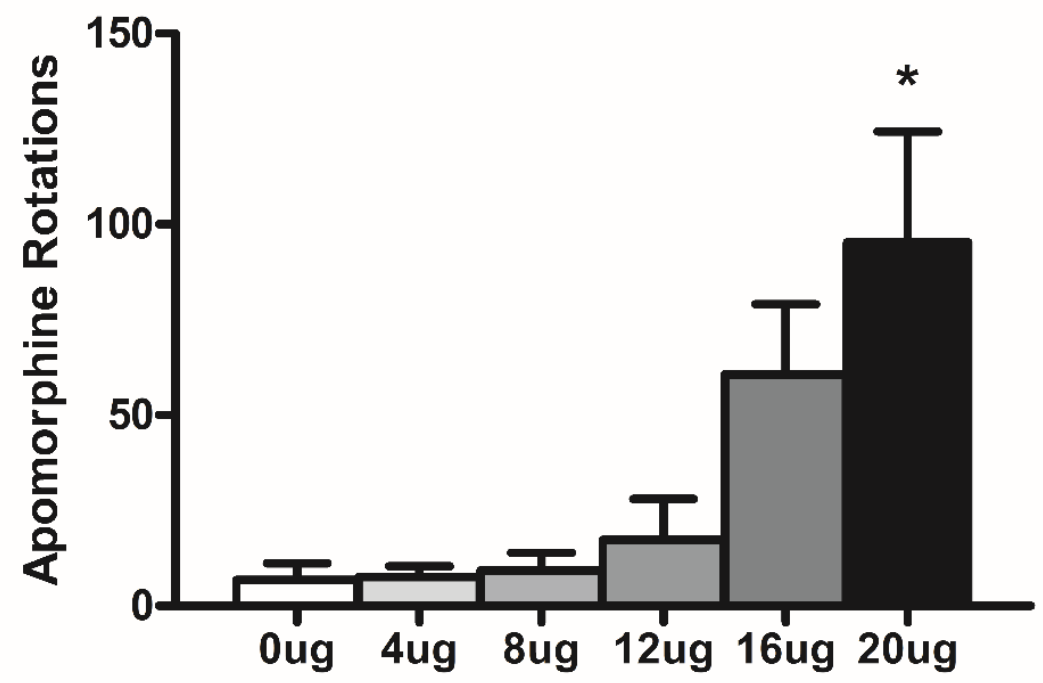

Figure 3 Apomorphine rotations (6-OHDA Titration)

Animals were given injections of apomorphine 30 days after 6-OHDA toxin administration. The net contra-lateral rotations over a 20 minute period were recorded. A one-way ANOVA found a significant treatment effect $(\mathrm{F}(5,23)=3.85, \mathrm{p}<.05)$. Post hoc analysis found a significant difference between the $20 \mu \mathrm{g}$ and the $0,4,8$, and $12 \mu \mathrm{g}$ groups $(\mathrm{p}<.05)$. 


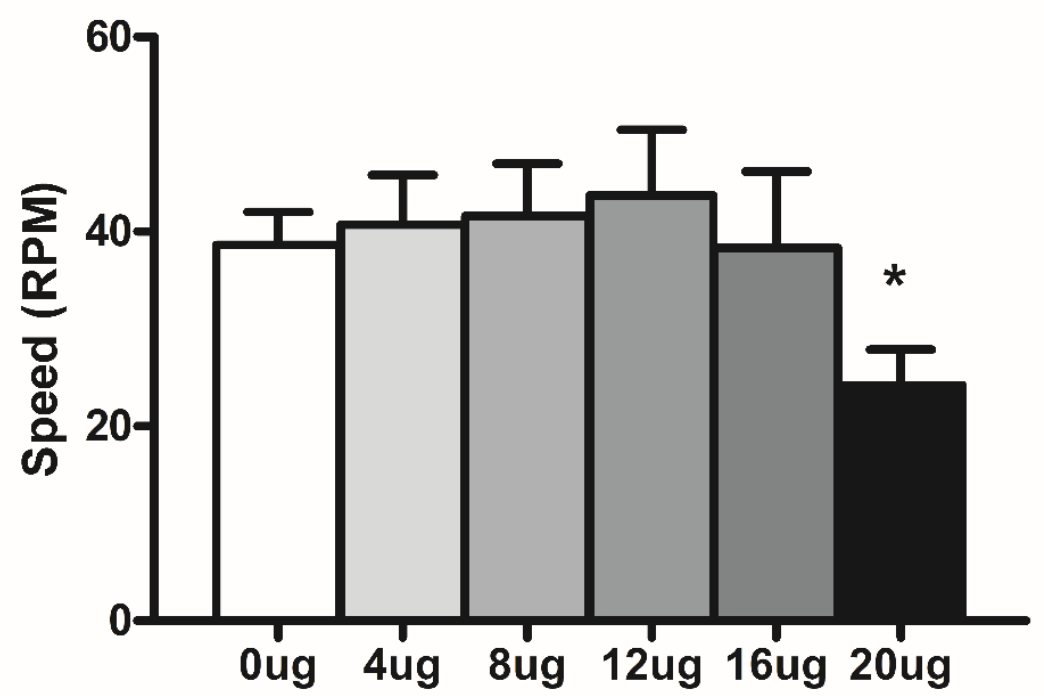

Figure 4 Rota Rod (6-OHDA Titration)

Animals were tested on the accelerating rota rod 30 days after 6-OHDA toxin administration. A one way ANOVA found a significant treatment effect $(\mathrm{F}(5,20)=2.74, \mathrm{p}<.05)$. Post hoc analysis found a significant difference between the $20 \mu \mathrm{g}$ and the $0,4,8$, and $12 \mu \mathrm{g}$ groups $(\mathrm{p}<.05)$. 


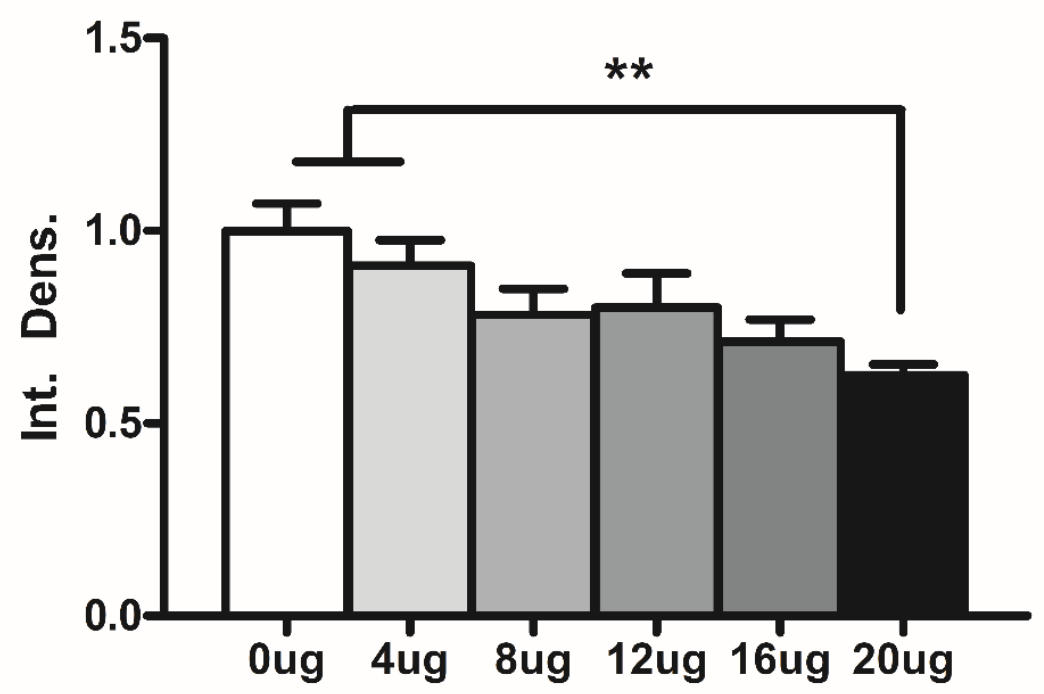

Figure 5 Striatal TH Integrated Density (6-OHDA Titration)

The integrated density of striatal sections immunolabelled with TH 30 days after intra-striatal administration of 6-OHDA. A one way ANOVA found a significant treatment effect $(F(5,22)=5.29, p<.01)$. Post hoc analysis found that the $0 \mu \mathrm{g}$ group was significantly different from the 8 , 12,16 , and $20 \mu \mathrm{g}$ groups $(\mathrm{p}<.05)$. Additionally, it was found that the $4 \mu \mathrm{g}$ group was also significantly different from the $20 \mu \mathrm{g}$ group $(\mathrm{p}<$ $.01)$. 


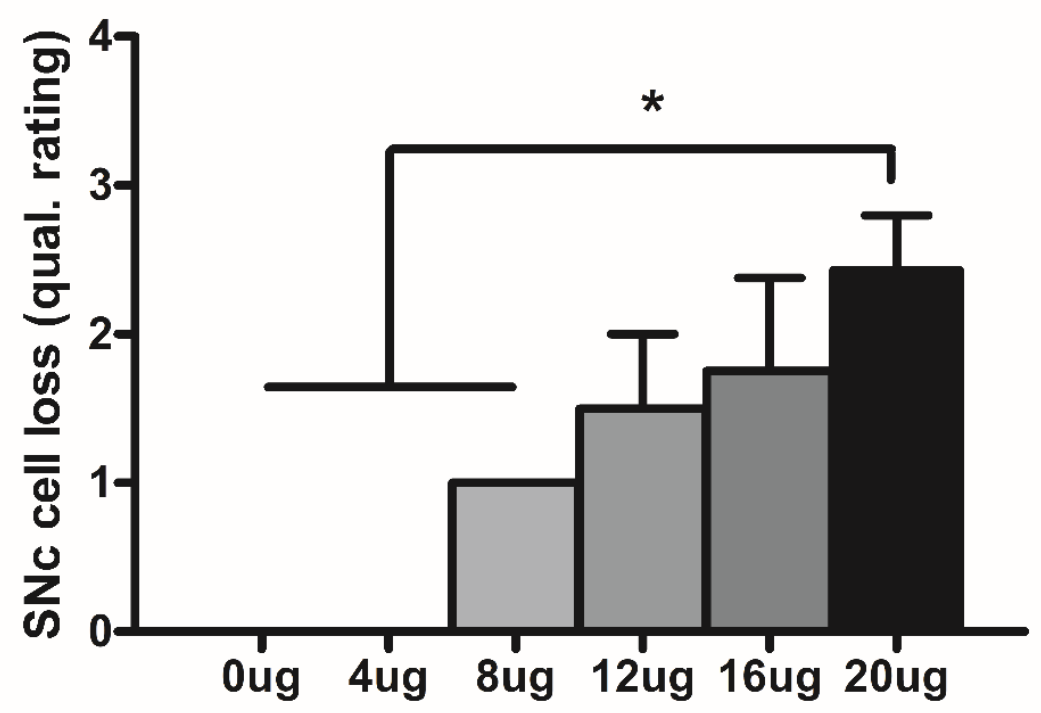

Figure 6 SNc cell loss (6-OHDA Titration)

A TH stain was performed on serial sections of the SNc 30 days after intra-striatal administration of 6-OHDA. A blind qualitative analysis comparing ipsilateral and contralateral sides was completed on the stained sections $(0=$ no noticeable cell loss, $1=$ minor cell loss, $2=$ approx. 50\% cell loss, $3=$ extensive cell loss). A one way ANOVA found a significant treatment effect $(\mathrm{F}(5,24)=9.98, \mathrm{p}<.0001)$. A post hoc analysis found that the 0 and $4 \mu \mathrm{g}$ were significantly different from the $8,12,16$, and $20 \mu \mathrm{g}$ groups $(\mathrm{p}<.05)$. Additionally, it was found that the $8 \mu \mathrm{g}$ was significantly different to the $20 \mu \mathrm{g}$ group $(\mathrm{p}<.05)$. 
The primary aim of this study was to complete a partial recapitulation of the rat model described by Blandini (2007) in a mouse. In adult Sprague Dawley rats Blandini found that $20 \mu \mathrm{g}$ of $6-\mathrm{OHDA}$ resulted in a lesion encompassing approximately $60 \%$ of the striatum, and greater than $50 \%$ cell loss in the substantia nigra. Blandini also found a robust rotational phenotype when the rats were challenged with apomorphine (Blandini et al., 2007). Surprisingly, the CD-1 mice required the same dosing of 6-OHDA to produce similar neuropathological and behavioural outcomes that are seen in the Sprague Dawley rats. Indeed, this dosing is fairly high compared to other mice strains. For example, we found that C57B16/J mice require approximately $12 \mu \mathrm{g}$ 6-OHDA to have similar degeneration (Ashley Thompson et. al, unpublished). Perhaps this is due to the outbred nature of the CD-1 strain compared to C57 mice. Other researchers have found that administering multiple smaller volume injections into different striatal regions can produce similar lesions with lower doses of 6-OHDA (Bagga et al., 2015). However, with more injection sites there is an increased risk of having a "surgical miss". Because of this it is preferential to use a larger concentration in a single injection. 
In general mice recover well from this procedure. We found that in the first $48 \mathrm{hrs}$ mice will lose $5-8 \%$ of their pre-surgical body weight. This weight loss can be prevented in most mice by providing access to a circulating water pad for at least the first $24 \mathrm{hrs}$ post-surgery. In some cases, supplementation with high caloric kitten replacement milk has proven beneficial in stimulating weight gain during the post-operative recovery. In addition to weight loss, mice treated with 6-OHDA present with spontaneous rotational behaviours when moving (animal is able to rest when they desire). Rotations are most pronounced in the first $72 \mathrm{hrs}$, reducing in severity as time progresses. In the vast majority of cases, mice fully recover within the first 7 days following administration of 6OHDA. Comparatively sham treated animals recover extremely well and display no overt behavioural symptoms. 


\title{
Conclusion
}

\author{
Unilateral administration of $20 \mu \mathrm{g}$ of 6-OHDA ( $\mathrm{HCl}$, Sigma
} H4381) into the striatum of CD-1 mice 6-8 week old mice produces a dopaminergic lesion similar to what would be expected in clinical PD. Similarly, this dose results in measurable behavioural deficits, providing opportunity to use a quantifiable measure of phenotypic improvement. Moving forward for the purpose of this thesis, these parameters will be used in all subsequent studies. 


\section{Description of Experiments}

This thesis aims to explore the potential role of CTEP as a neurorecovery agent in a 6-OHDA toxin model of Parkinson's disease. In all described experiments animals received $20 \mu \mathrm{g}$ of $6-\mathrm{OHDA}$ into the right anterior striatum. In all experiments, we waited a full 30 days before administering any pharmacological manipulation to ensure a maximal striatal lesion. Furthermore, prior to any drug administration all animals are challenged with apomorphine to confirm a parkinsonian phenotype. If there is indeed a significant unilateral lesion the animals will rotate in a contralateral direction - an indication of dopaminergic de-innervation and dopamine receptor hyperactivity. In all described experiments CTEP was administered at a dose of $2 \mathrm{mg} / \mathrm{kg}$ given every $48 \mathrm{hrs}$.

In one experiment, we wished to determine any short term effects of CTEP treatment. As such, CTEP was given over a period of 1 week. The first administration was given via intraperitoneal injection. Four hours following this bolus administration of CTEP the animals were challenged with apomorphine. This was done to determine if 
CTEP had any immediate effects on the parkinsonian phenotype. Following this behavioural test, mice were returned to their home cages and continued receiving CTEP via a chocolate pudding suspension. Indeed, this was the only time CTEP was administered by injection. All other administrations of CTEP (in this study and all others) was given via a pudding suspension. On the last day of the experiment - day 7 of CTEP treatment - the mice were tested on an accelerating rota rod, followed by another apomorphine induced rotation challenge. After this last behavioural test, the mice were euthanized. These behaviours were timed to measure the behavioural phenotype after a full 7 days of CTEP treatment. In this experiment, striatal $\mathrm{TH}+$ fibre density was assessed through histology to determine any effects of CTEP on overall lesion size. Additionally, downstream signaling partners of mGluR5 were measured using Western blot in both the striatum and motor cortex. Both these regions have high expressions of the mGluR5 receptor and form two distinct regions of the basal ganglia motor circuit. As such, it is of interest to investigate if CTEP modulates mGluR5 differently in distinct brain regions. Additionally, changes in potential trophic factors were measured in striatal lysates - the site of 6-OHDA induced degeneration. 
In a second experiment we wished to determine any long term effects of CTEP treatment. As such, following a 6-OHDA induced lesion CTEP was given over a 12 week period via a chocolate pudding suspension. Near the end of the experiment, animals were tested in a modified reversal Morris water maze to measure cognitive flexibility. On the last day of the experiment mice were tested on an accelerating rota rod, followed by another apomorphine induced rotation challenge, and then subsequently euthanized. Similar to the previous experiment, striatal $\mathrm{TH}+$ fibre density was measured through histology to determine the impact of CTEP on striatal lesion. Additionally, a stereological count of $\mathrm{TH}+$ cells was completed to determine if CTEP may have any neurogenic properties. In this experiment, additional histology was completed assessing FosB + cells. In this experiment, FosB was used as an indicator of overall post synaptic activity. Again, the downstream signaling partners of mGluR5 and potential trophic factors were measured using Western blot in both the striatum and motor cortex.

In a third experiment we wished to determine if any effects of CTEP persist after the drug is no longer being administered. To address this, a partial recapitulation of the 1 week CTEP study was completed. 
However in this experiment, following the 1 week of drug administration the animals were given an additional 1 week drug washout before being euthanized. In this small study, the only behaviour assessed was the apomorphine induced rotations. Animals were challenged with apomorphine 30 days following the infusion of 6OHDA (to confirm parkinsonian phenotype), after 1 week of CTEP administration (to confirm CTEP induced improvements), and once again after 1 week of drug washout (to determine if the observed changes persist). For this small study, only striatal $\mathrm{TH}+$ fibre densities were measured.

In the previous experiments, we identified the mTOR signaling cascade (more specifically mTORC1) as being a potentially important mediator of CTEP induced changes in the 6-OHDA toxin model. To confirm the role of mTORC1, a fourth experiment was carried out. A partial recapitulation of the 1 week study was completed, however in addition to CTEP treatment some animals also received injections of rapamycin - a potent inhibitor of mTORC1. In this study animals received an i.p. injection of rapamycin every $48 \mathrm{hrs}$ in the evenings ( 16hrs) before CTEP administration. This was timed so that rapamycin 
had sufficient time to be absorbed, cross the blood brain barrier, and to inhibit mTORC1 before CTEP could begin to activate it. In this study animals were only tested behaviourally with apomorphine. $\mathrm{TH}+$ fibre densities were measured to determine the extent of striatal lesion. Finally, downstream effectors of mTOR were measured to confirm that indeed rapamycin did inhibit the protein.

Together these experiments aimed to determine: (A) whether CTEP had any immediate behavioural effects, (B) whether CTEP had any effects after a short 1 week administration period, (C) whether CTEP had any effects after a long 12 week administration period, (D) whether the effects of CTEP are permanent or are susceptible to regression, (E) to identify a potential mechanism identifying how CTEP may be working, and (F) to determine if CTEP acts equally/similarly in different brain regions. 


\title{
Experimental Methods
}

\author{
$\underline{\text { Animals }}$ \\ Male CD-1 mice sourced from Charles River (Kingston, Ontario, \\ Canada), aged 6-8 weeks upon delivery were used for all described \\ experiments. Animals were individually housed in techniplast blue line \\ individually ventilated polypropylene cages (approx. dimensions: \\ 400x200x200mm) with no enrichment other than nestlets. Animals were \\ maintained on a standard $12 \mathrm{~h}$ light/dark cycle. Tap water and chow \\ (Teklad, \#2014) was provided ad libitum, while room temperature and \\ humidity were maintained at $20^{\circ} \mathrm{C}$ and $50 \%$, respectively. All aspects of \\ this experiment were approved by the Carleton University Committee \\ for Animal Care and adhered to the guidelines outlined by the Canadian \\ Council for the Use and Care of Animals in Research.
}

\section{Surgery}

The mice were anaesthetized using variable flow isoflurane inhalational anesthetic $\left(1.5-5 \%\right.$ in pure $\left.\mathrm{O}_{2}\right)$. Animals were then placed in a Kopf instruments Model 940 stereotaxic frame (Kopf Instruments, Tujunga, CA, USA). An L-shaped PlasticsOne 3280 P cut $3 \mathrm{~mm}$ below the 
pedestal was inserted into the right striatum at the coordinates $1.00 \mathrm{~mm}$ anterior, $1.75 \mathrm{~mm}$ lateral, and 3.00 ventral relative to bregma and skull surface. A single $2 \mu \mathrm{l}$ infusion of vehicle solution (in $0.9 \%$ saline solution containing $0.02 \%$ ascorbic acid) or 6-hydroxydopamine hydrochloride $(20 \mu \mathrm{g}$, Sigma-Aldrich, H4381) was administered at a rate of $0.4 \mu 1 / \mathrm{min}$ using a Harvard apparatus picoplus pump equipped with a $10 \mu 1$ Hamilton syringe. The cannula was retracted after a 5 minute incubation period. Bonewax (Stoelting, Wood Dale, IL, USA) was placed over the drill hole and the incision closed using non-absorbable nylon sutures. Animals were given tramadol $(20 \mathrm{mg} / \mathrm{kg}$ at $2.5 \mathrm{mg} / \mathrm{ml}$, Chiron Compounding Pharmacy, Guelph, ON, Canada) twice a day for 3 days, beginning on the day of surgery. Mice were given a 30 day period before any pharmacological manipulation to ensure a maximal 6OHDA induced lesion.

\section{Pudding training}

We found that pudding was the best vehicle to deliver CTEP in a rapid and highly reliable manner. Starting 3 weeks after the surgery, mice were introduced to our pudding administration regimen. During the 7 day training period mice were given daily access to $0.1 \mathrm{ml}$ chocolate pudding (Loblaws No Name Chocolate Pudding Cups, 
20297693001_EA) on a plastic petri dish (Falcon, 35x10mm) starting immediately when the lights turn on in the morning. Day 1 mice were allowed free access for 10 minutes. Days 2-7 the dish was removed immediately after the mouse consumed the pudding. By the end of the training regimen most mice consume all their pudding within 1 minute.

\section{CTEP}

Thirty days following the surgery, mice were given either vehicle or CTEP (Axon Medchem, Axon 1972) every 48hrs at 2mg/kg, starting immediately when the lights turn on in the morning. For the acute (1 week) study mice were given a single bolus injection $(0.4 \mathrm{mg} / \mathrm{ml}, 0.9 \%$ saline with $1.6 \%$ DMSO) of CTEP or vehicle, followed by 3 administrations as a pudding suspension $(1 \mathrm{mg} / \mathrm{ml})$. For the chronic (12 week) study mice were given all doses as a pudding suspension $(1 \mathrm{mg} / \mathrm{ml})$.

\section{$\underline{\text { Rapamycin }}$}

In a subset of experiments, some mice were given either vehicle $(5 \%$ Polyethylene glycol 400 [Sigma-Aldrich, 91893] and 5\% Tween-80 [Sigma-Aldrich, P1754] in distilled water) or Rapamycin $(6 \mathrm{mg} / \mathrm{kg}$ at 1mg/ml, LC Laboratories R-5000, Massachusetts, USA). Rapamycin 
was administered via intraperitoneal injections every $48 \mathrm{hrs}$, in the evening preceding CTEP administration.

\section{Drug Washout}

In a subset of experiments, some mice were given CTEP or vehicle as previously described for 1 week, followed by a 1 week drug washout period. This study assessed whether the effects of CTEP might persist beyond the administration timeframe.

\section{Behavioural testing}

Prior to each behavioural test, mice were habituated to the testing room for at least a 20 minute period.

\section{Apomorphine Induced rotations}

Animals were administered apomorphine hydrochloride hemihydrate (sub cutaneous injection, $0.5 \mathrm{mg} / \mathrm{kg}$ at $0.1 \mathrm{mg} / \mathrm{ml}$ in $0.9 \%$ saline, SigmaAldrich, A4393) 30 days following the surgical procedure to confirm parkinsonian phenotype. Any mice that received 6-OHDA and did not demonstrate net contralateral rotations were removed from the study. Briefly, immediately following the injection of apomorphine animals were placed into large open container $(27 \times 48 \times 20 \mathrm{~cm})$ and recorded from above. After a 5 minute drug incubation period, net contralateral 
rotations were counted over a 20 minute period. For the 1 week CTEP study mice were challenged with apomorphine on 3 occasions - 30 days after surgery, 4 hours following the bolus IP injection of CTEP, and after 1 week of CTEP administration. For the 12 week CTEP study, mice were challenged with apomorphine on 2 occasions - 30 days after surgery, and again after the 12 weeks of CTEP administration. For the washout study, mice were challenged on 2 occasions - 30 days after surgery, and again after the drug washout period. For the rapamycin study, mice were challenged on 2 occasions - 30 days after surgery, and again after 1 week of CTEP/rapamycin treatments.

\section{Accelerating Rota Rod}

Animals were trained on the rota rod (Omnitech Electronics) over a 2 day period. On day one animals were placed on the rota rod apparatus which was maintained at a continual speed of 11RPM. If the animal fell from the apparatus they were immediately returned over a 5 minute period. This process was repeated three times total with an inter-trial interval of 60 minutes. Day two was a repeat of Day 1, however the rota rod was maintained at 22RPM. On the third day animals were tested using an accelerating protocol with a rotation speed increasing from 4 RPM to 88 RPM over a 600 second period. Maximum speed achieved 
before falling off the apparatus was recorded. Animals were tested over 3 trials. The lowest of the 3 trials was discarded and the average of the remaining 2 trials were used as the animals scored. The test day coincided with the last day of the experiment. To reduce test-retest confounds, animals were only tested on the rota rod on the last day of experimentation.

\section{Modified Reversal Morris water maze}

This protocol has been adapted from previously used methods by Nunez (2008) and Salmaso (2012). Only mice in the 12 week CTEP administration study were tested in the water maze. Briefly, our water maze was located in a dimly lit room. On each wall were unique identifiers for orientation. Mice received 2 days of training, the first of which occurred 2 weeks prior to experiment end, and the second of which occurred 4 days prior to experiment end. Mice were introduced into the pool from the same entry point for each trial. The platform ( $8.25 \mathrm{~cm}$ in diameter submerged $\sim 0.5 \mathrm{~cm}$ under the water surface) remained in a fixed location during the training period. Mice received two sets of 6 training trials, separated by a $1 \mathrm{hr}$ break and an inter-trial interval of 10minutes. The trial ended either when the mouse found the platform or 60 seconds had elapsed. Mice that did not find the platform 
were directed to platform before being removed from the pool. Immediately following the 12 th trial on training day 2 , mice were tested in the probe trial, where the platform was moved to the opposite quadrant. Time to find the platform was recorded using behavioural tracking software (ANY-maze, Stoelting). This behavioural test is a measure of cognitive flexibility.

\section{Euthanization}

Animals were randomly assigned into one of two groups - histology or western blot. Animals that were assigned to the histology group were deeply anesthetized with an overdose of sodium pentobarbital. Mice were then transcardially perfused using chilled $0.9 \%$ saline followed by 4\% PFA solution in $0.1 \mathrm{M}$ phosphate buffer. Extracted brains were post fixed for $24 \mathrm{hrs}$ in $4 \%$ PFA, followed by sequential washes of $10 \%$ sucrose in $0.1 \mathrm{M}$ phosphate buffer over another $24 \mathrm{hr}$ period. Tissue was then incubated in a $30 \%$ sucrose solution in $0.1 \mathrm{M}$ phosphate buffer for 72hrs. Following this last sucrose wash tissue was flash frozen and stored at $-80^{\circ} \mathrm{C}$. Animals that were assigned to the western blot group were euthanized by rapid decapitation. Extracted brain tissues were immediately micro dissected, placed on dry ice, and stored at $-80^{\circ} \mathrm{C}$. 


\section{Histology}

Tissue was sectioned at $40 \mu \mathrm{m}$ on a rotary cryostat. Every $3^{\text {rd }}$ serial striatal section and every $2^{\text {nd }}$ serial nigral section was separated for histology. Free floating tissue sections were immuno-labelled overnight in primary antibodies (mouse anti-TH 1:2000 [Immunostar 22941], rabbit anti-FosB [Cell Signalling Technology [2251]). Tissue sections were incubated in biotinylated secondary and tertiary streptavidin HRP solutions for $2 \mathrm{hrs}$ each. Whole section images of all histological sections were captured at 10x magnification using a Zeiss axio imager M2 or ThermoFisher EVOS FL Auto 2. TH+ labelled striatal sections were quantified using ImageJ software. The integrated densities of the entire striatal area of the slices at the site of 6-OHDA injection and the two adjoining sites (spanning approximately $280 \mu \mathrm{m}$ ) were measured as a ratio to its ipsilateral hemisphere signals after cortical background subtraction. The values of the three sites were averaged and recorded. Four slices of FosB immunolabelled tissue, spanning equally before and after 6-OHDA infusion site were selected for analysis. FosB immunolabelled nuclei were automatically counted using ImageJ particle analysis (size parameter $20-300 \mu \mathrm{m}^{2}$, circularity $0.2-1.00$ ). The average FosB + cells within the striatum or motor cortex of the 4 slices 
measured were recorded. For TH labelled SNc sections, a stereological count of $\mathrm{TH}+$ neurons in every $2^{\text {nd }}$ slice spanning the entire $\mathrm{SNc}$ was completed using the optical fractionator probe at $63 \mathrm{x}$ on a Zeiss axio imager M2 and MBF StereoInvestigator10.

\section{Western Blots}

Protein from microdissected tissue samples stored at $-80^{\circ} \mathrm{C}$ was extracted using a RIPA like extraction buffer with EDTA-free protease inhibitors (Roche cOmplete) followed by treatment with concentrated Laemmli buffer. Following extraction, samples were then stored at $20^{\circ} \mathrm{C}$. Western gels were run in reducing conditions at $140 \mathrm{~V}$ and transferred onto $\mathrm{MeOH}$ activated PVDF membranes at 100V. Primary antibodies used include from Cell Signalling Technology (phosho-p70 S6 Kinase (Thr389)[9205], phosphor-mTOR (Ser2448)[5536]), Santa Cruz Biotechnology (p-ERK(E-4 Tyr204)[sc-7383], p-CaMKII $\alpha$ (Thr286)[sc-12886-R]), and R\&D Systems (BDNF [MAB248]). Signal was visualized with either ECL chemiluminescence and X-ray film exposure or using tagged fluorophores and the Licor Odyssey FC imaging system. For western blots completed with chemiluminescence membranes were treated with skim milk $(\leq 0.5 \%)$ in TBST based 
solutions and normalized $\beta$-actin signal. For western blots completed with tagged fluorophores membranes were treated with fishgelatin $(\leq$ $0.5 \%$ ) in TBST based solutions, and normalized to total protein signal as measured using a fast green stain.

\section{Statistics}

One-way, Two-way, or Repeated-measurement ANOVAs were completed as appropriate. Post hoc testing was completed using Fisher's PLSD. 


\section{Experiment 1}

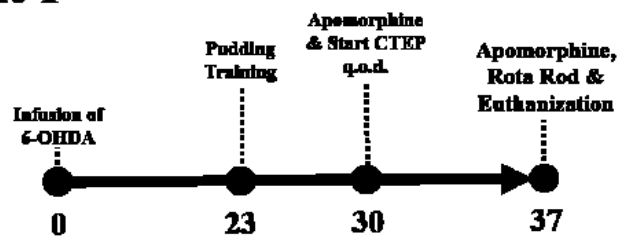

\section{Experiment 2}

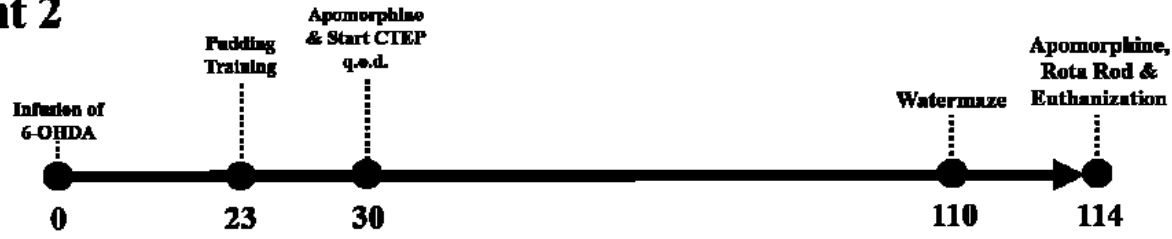

\section{Experiment 3}

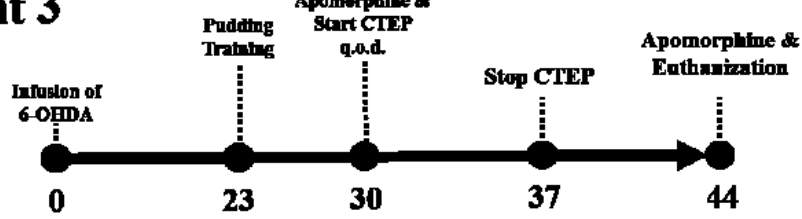

\section{Experiment 4}

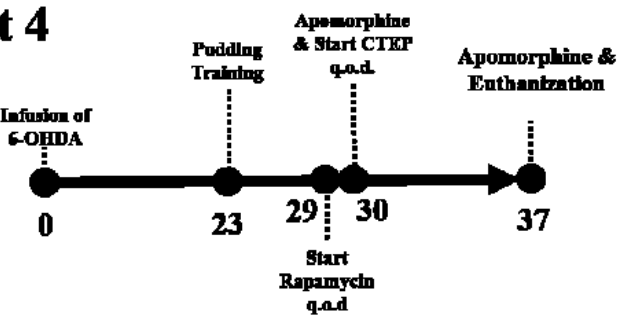

Figure 7 CTEP Experimental Time-courses

Experimental time-courses for the 4 different experiments carried out using CTEP. (1) 1 week CTEP administration, (2) 12 week CTEP administration, (3) 1 week CTEP with additional 1 week drug washout, and (4) 1 week CTEP with paired Rapamycin treatment injections. 


\section{Table 2 Methods - CTEP Experimental Group Designs}

\section{(A) 1 Week CTEP Administration}

\begin{tabular}{|c|c|c|}
\cline { 2 - 3 } \multicolumn{1}{c|}{} & Vehicle & CTEP \\
\hline 6-OHDA & & \\
\hline Sham & & \\
\hline
\end{tabular}

Behaviour $\mathrm{n} \approx 6$ /group

Western $\mathrm{n} \approx 4$ /group

Histology $\mathrm{n} \approx 5 /$ group

(B) 12 Week CTEP Administration

\begin{tabular}{|c|c|c|}
\cline { 2 - 3 } \multicolumn{1}{c|}{} & Vehicle & CTEP \\
\hline 6-OHDA & & \multicolumn{1}{c|}{} \\
\hline Sham & & \multicolumn{1}{c}{} \\
\hline
\end{tabular}

Behaviour $\mathrm{n} \approx 12 /$ group

Western $\mathrm{n} \approx 5$ /group

Histology $\mathrm{n} \approx 5 /$ group

(C) 1 Week CTEP Administration + 1 Week Drug Washout

\begin{tabular}{|c|c|c|}
\cline { 2 - 3 } \multicolumn{1}{c|}{} & Vehicle & CTEP \\
\hline 6-OHDA & & \\
\hline Sham & & \multicolumn{1}{|c}{} \\
\hline
\end{tabular}

Behaviour $\mathrm{n} \approx 10$ /group

Western $\mathrm{n} \approx 5$ /group

Histology $\mathrm{n} \approx 5 /$ group

(D) 1 Week CTEP + Rapamycin Administration

\begin{tabular}{|c|c|c|c|c|}
\hline & \multicolumn{2}{|c|}{ Vehicle } & \multicolumn{2}{|c|}{ Rapamycin } \\
\hline & Vehicle & CTEP & Vehicle & CTEP \\
\hline \multicolumn{5}{|l|}{ 6-OHDA } \\
\hline Sham & & & & \\
\hline $\begin{array}{l}\text { Behaviour } \\
\text { Western } \mathrm{n} \\
\text { Histology } \mathrm{r}\end{array}$ & $\begin{array}{l}\text { 12/group } \\
\text { group } \\
\text { 5/group }\end{array}$ & & & \\
\hline
\end{tabular}




\section{Results}

\section{Week of CTEP Administration: Behaviour}

In this experiment we wished to determine any short term effects of CTEP treatment. As such, CTEP was given over a period of 1 week. Prior to CTEP administration, there was a robustly significant increase in apomorphine induced rotations in animals that received 6-OHDA 30 days earlier $(\mathrm{F}(1,18)=232.70, \mathrm{p}<.0001)$. The following day, animals were given a single bolus injection of CTEP or vehicle and challenged with apomorphine 4 hours later. This was done to determine if CTEP had any immediate effects on the parkinsonian phenotype. A two-way ANOVA found no significant 6-OHDA x CTEP interaction, however as expected, the main effect for 6-OHDA was still evident $(\mathrm{F}(1,18)=$ 187.00, $\mathrm{p}<.0001)$. Comparatively, CTEP administration had no significant effect after this single injection $(\mathrm{F}(1,18)=0.33, \mathrm{p}=.57)$ (Figure 8). Following this test, animals then continued to receive the CTEP in the aforementioned pudding suspension every 48hrs for remainder of the 1 week period. On the last day of the experiment (Day 
37 following 6-OHDA), mice were tested on an accelerating rota rod to assess motor coordination. On this test, rota rod performance (ie the RPM at which mice fell off the rotating drum) varied as a function of a CTEP X 6-OHDA interaction $(F(1,18)=5.41, \mathrm{p}<0.05)$ (Figure 9). The follow up comparisons revealed that CTEP did indeed reverse the effects of 6-OHDA, such that mice remained on the apparatus longer than those that did not receive the drug and in fact were equivalent to sham mice $(p<0.001)$. Following their test on the rota rod, the mice were then again given apomorphine, and rotations were assessed once again. The two-way ANOVA revealed a significant CTEP X 6-OHDA interaction $(F(1,18)=12.24, \mathrm{p}<0.01)($ Figure 8$)$. The post hoc analysis found that mice that received CTEP and the 6-OHDA lesion had significantly less rotations than those that had the lesion in the absence of the drug $(\mathrm{p}<.01$, respectively) (Figure 8$)$. 


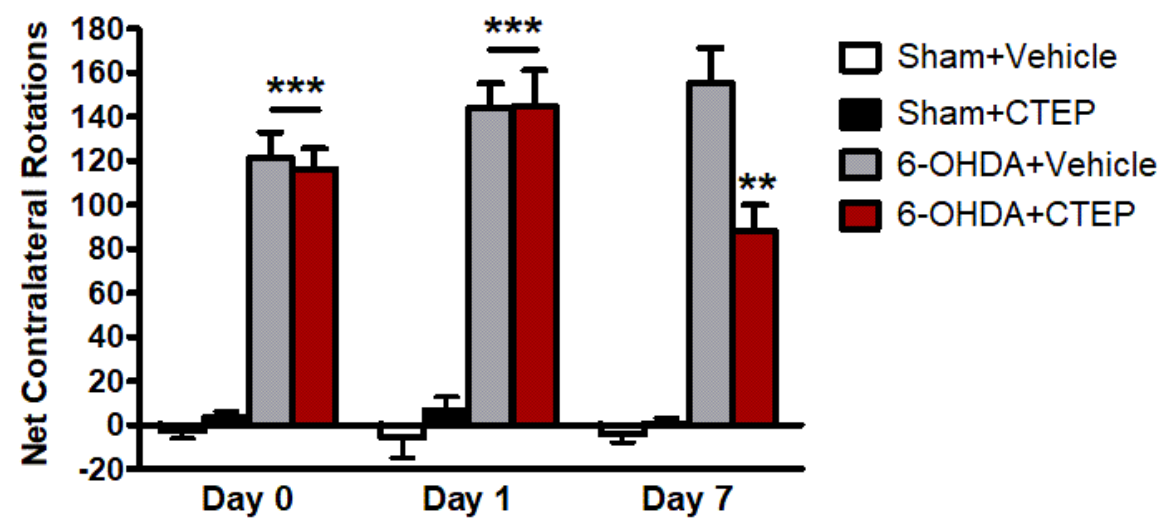

Figure 8 Apomorphine Induced Rotations (1 week CTEP)

Animals were given injections of apomorphine 30 days after 6-OHDA administration. The net contra-lateral rotations over a 20 minute period were recorded. A two-way ANOVA found that at Day 30, all animals treated with 6-OHDA demonstrated significant rotational behaviour $(\mathrm{F}(1,18)=232.70, \mathrm{p}<.0001)$. After a bolus administration a CTEP and subsequent apomorphine challenge 4 hours later a two-way ANOVA found a significant effect of 6-OHDA $(\mathrm{F}(1,18)=187.00, \mathrm{p}<.0001)$ but no effect of CTEP $(\mathrm{F}(1,18)=0.33, \mathrm{p}=.57)$. After 1 week of CTEP administration a two-way ANOVA found a significant CTEP $\mathrm{x}$ 6OHDA interaction $(\mathrm{F}(1,18)=12.24, \mathrm{p}<.01)$. Follow up comparisons found that 6-OHDA+CTEP was significantly different from 6OHDA+Vehicle $(\mathrm{p}<.01)$ as well as Sham+Vehicle and Sham+CTEP $(\mathrm{p}$ $<.0001$, respectively). 


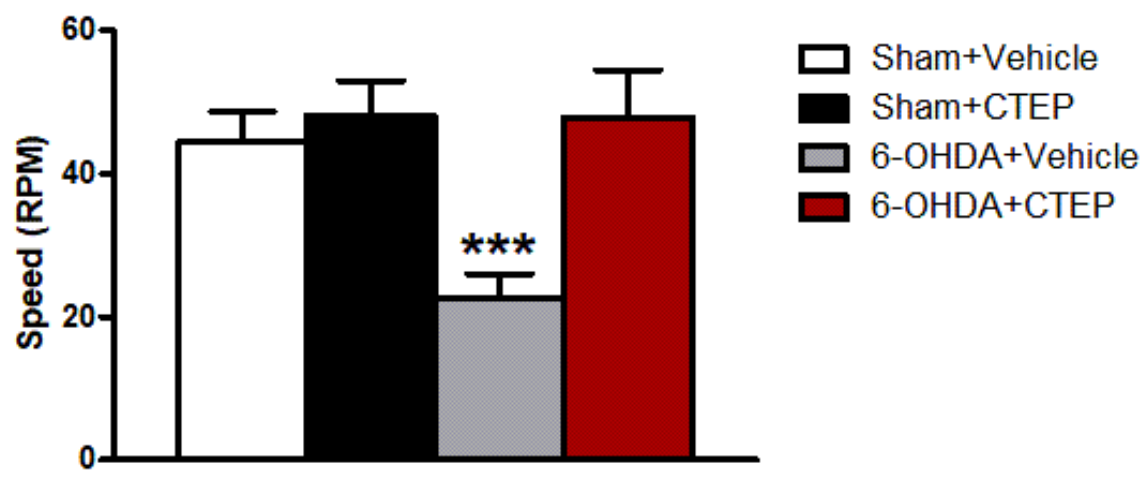

Figure 9 Rota Rod (1 week CTEP)

Animals were tested on the accelerating rota rod on day 37 of the experiment after 1 week of CTEP administration. A two way ANOVA found a significant CTEP x 6-OHDA interaction $(\mathrm{F}(1,18)=5.41, \mathrm{p}<$ $.05)$. Post hoc analysis found a significant difference between the 6OHDA+Vehicle treatment group and all other treatment groups $(\mathrm{p}<$ $.001)$. 


\section{Week of CTEP Administration: Molecular}

Animals in this 1 week study were randomly divided into one of two groups; (A) Histology or (B) Western Blot. Animals in the histology group were perfused as previously described. Striatal sections were immunohistochemically assessed for the density of $\mathrm{TH}+$ fibres as an indicator of overall dopaminergic striatal damage. A significant two way interaction between CTEP and 6-OHDA was evident for striatal $\mathrm{TH}+$ density $(F(1,15)=7.25, \mathrm{p}<.05)($ Figure 10$)$. Not surprisingly, the post hoc analysis found that 6-OHDA treatment significantly reduced $\mathrm{TH}+$ staining, relative to Sham treated animals $(\mathrm{p}<.01)$. Importantly, among the 6-OHDA lesioned mice, CTEP significantly increased $\mathrm{TH}+$ staining $(\mathrm{p}<.05)$.

Western blotting was used to measure mGluR5 related proteins in the striatum. First, levels of p-ERK1/2(Tyr204), pCaMKII $\alpha(T h r 286)$, and p-mTOR(Ser2448) were measured, as these are important downstream effectors of mGluR5. There was no significant difference in p-ERK1/2(Tyr204) regardless of CTEP or 6-OHDA treatments, $\left(F^{\prime} s(1,14)<1, p>0.05\right)$ (Figure 11). However, p- 
CaMKII $\alpha($ Thr286) levels did vary as a function of the CTEP x 6-OHDA treatments $(\mathrm{F}(1,14)=6.74, \mathrm{p}<.05)($ Figure 12). Further, the follow up analyses revealed that while 6-OHDA alone significantly reduced pCaMKII $\alpha($ Thr286) levels in the striatum, CTEP completely reversed this effect to a level comparable to sham treatments $(p<.05)$.

In the absence of an interaction effect, the 6-OHDA treatment increased striatal p-mTOR $(\operatorname{Ser} 2448)$ levels $(F(1,12)=5.49, p<.05)$. But as can be seen in Figure 12, this elevation was confined to the CTEP treated mice. Indeed, although the CTEP main effect just missed significance, $(\mathrm{F}(1,12)=3.57, \mathrm{p}=.08)$, clearly this drug selectively increased p-mTOR(Ser2448) levels in the context of the lesion (Figure 13). The downstream target of mTOR, p-p70S6K(Thr389), varied as a function of the CTEP $\times$ 6-OHDA treatments $(\mathrm{F}(1,12)=9.14, \mathrm{p}<.05)$ (Figure 14). Post hoc analysis confirmed that CTEP increased striatal pp70S6K(Thr389) when given in the context of the 6-OHDA lesion, relative to the remaining three groups $(\mathrm{p}<0.01)$.

$\mathrm{mTOR} / \mathrm{p} 70 \mathrm{~S} 6 \mathrm{~K}$ together stimulate protein translation. One known product of increase mTOR activity is BDNF. As such, the trophic 
factor BDNF was assessed as a potential mediator of the beneficial effects imparted by CTEP treatment. Significant main effects were apparent for both the 6-OHDA $(\mathrm{F}(1,12)=6.61, \mathrm{p}<.05)$ and CTEP $(\mathrm{F}(1,12)=6.15, \mathrm{p}<.05)$ treatments with regards to levels of striatal BDNF, however no interaction was found. As shown in Figure 15, CTEP selectively increased striatal BDNF in mice that also had the 6OHDA lesion, relative to the remaining groups $(\mathrm{p}<.01)$.

Another region with high mGluR5 expression is the cerebral cortex. It is of interest to identify any changes in mGluR5 signaling to determine if they duplicate what is seen in the striatum, or if perhaps CTEP alters receptor signalling differentially. Within the motor cortex, p-ERK1/2(Tyr204) levels significantly varied as a function of the CTEP x 6-OHDA treatments $(\mathrm{F}(1,14)=7.88, \mathrm{p}<.05)($ Figure 16). The post hoc analysis revealed that 6-OHDA administration caused a marked reduction of cortical p-ERK1/2(Tyr204) levels $(\mathrm{p}<.001)$, but that CTEP treatment completely reversed this effect. A significant CTEP x 6OHDA interaction was also evident with regards to $\mathrm{p}$ CaMKII $\alpha($ Thr286) cortical levels $(\mathrm{F}(1,14)=17.99, \mathrm{p}<.001)$ (Figure 17). Once again, the follow up comparisons indicated that 6-OHDA 
reduced cortical p-CaMKII $\alpha($ Thr286) $(\mathrm{p}<.05)$ and that CTEP treatment reversed this effect. Finally, when cortical p-mTOR(Ser2448) levels were assessed a different pattern of effects emerged. Specifically, in the absence of an interaction, there were significant main effects for 6$\operatorname{OHDA}(\mathrm{F}(1,12)=6.10, \mathrm{p}<.05)$ and $\operatorname{CTEP}(\mathrm{F}(1,12)=4.80, \mathrm{p}<.05)$ treatments (Figure 18). The post hoc analysis revealed that CTEP alone in the absence of 6-OHDA increased p-mTOR(Ser2448) relative to all other treatment groups $(\mathrm{p}<.05)$. 


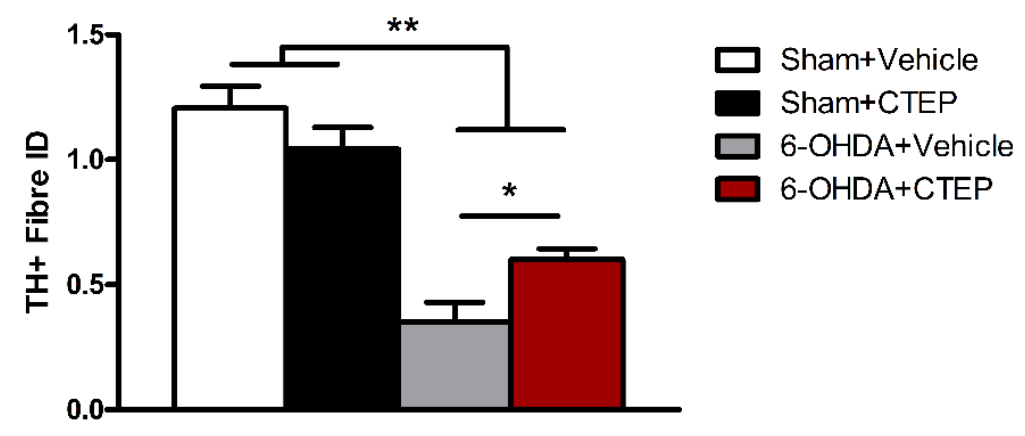

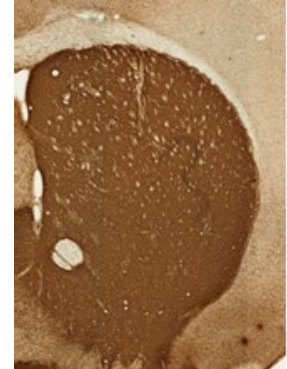

Shen+Veh

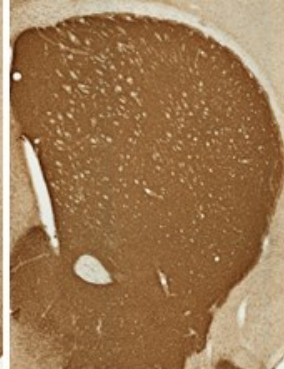

Shan+CTEP

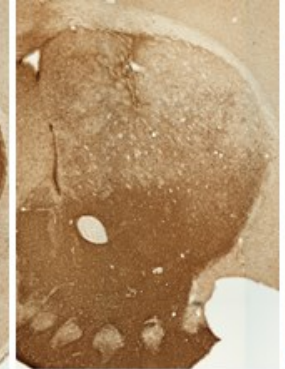

6-OHDA+YEH

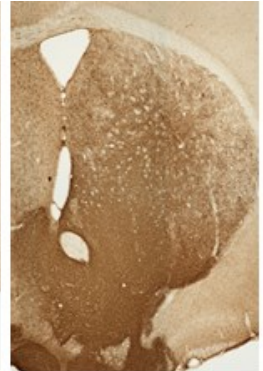

6-OHDA+CTEP

\section{Figure 10 Striatal TH+ Histology (1 week CTEP)}

The integrated density of striatal sections immunolabelled with TH was measured using ImageJ software after 1 week of CTEP administration. A two way ANOVA found a significant CTEP x 6-OHDA interaction $(F(1,15)=7.25, p<.05)$. Post hoc analysis found that animals treated with 6-OHDA had significantly less $\mathrm{TH}+$ staining that Sham treated animals $(\mathrm{p}<.01)$. Additionally, 6-OHDA+CTEP treated animals had significantly more $\mathrm{TH}+$ staining compared to 6-OHDA+Vehicle treated animals $(\mathrm{p}<.05)$. 

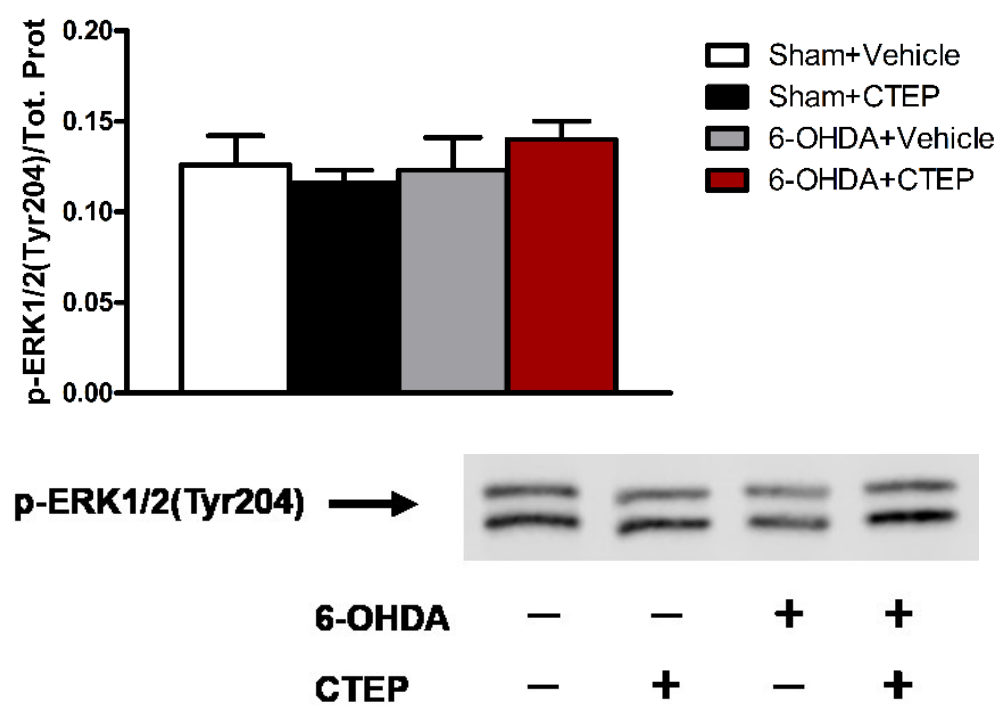

Figure 11 Striatal p-ERK1/2(Tyr204) (1 week CTEP)

Striatal p-ERK1/2(Tyr204) was measured after 1 week of CTEP administration by western blot. A two-way ANOVA found no main effects, or interactions $\left(F^{\prime} s(1,14)<1, p>.05\right)$. This western blot was visualized using IR800 fluorophore on Licor Odyssey FC equipment, normalized total protein signal following a Fast Green Stain. 

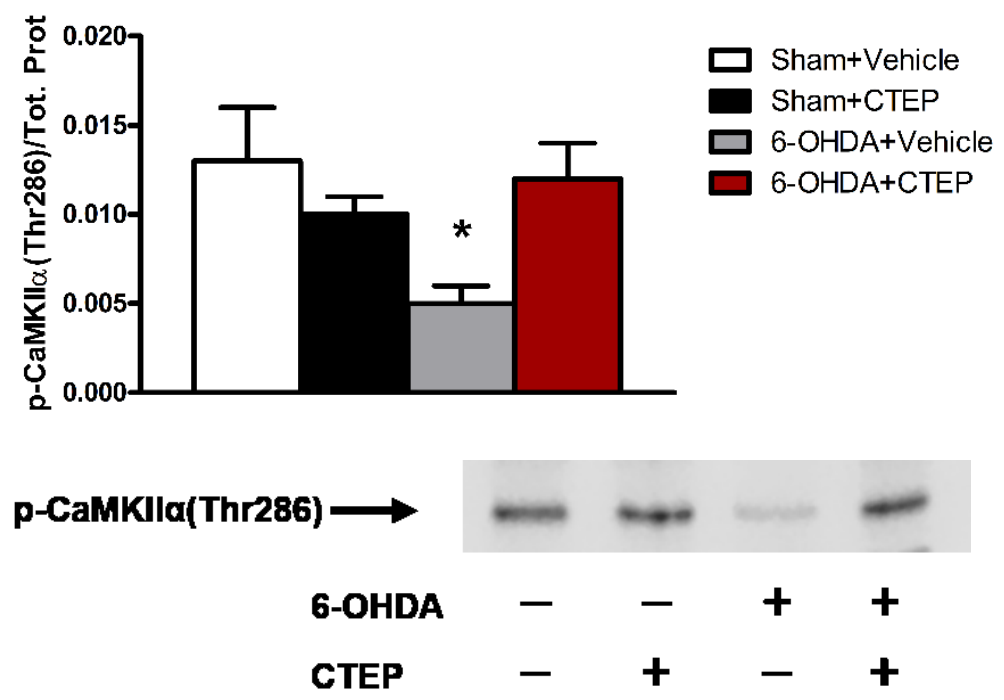

Figure 12 Striatal p-CaMKII $\alpha(\operatorname{Thr} 286)$ (1 week CTEP)

Striatal p-CaMKII $\alpha($ Thr286) was measured after 1 week of CTEP administration by western blot. A two-way ANOVA found a significant CTEP $\times$ 6-OHDA interaction $(\mathrm{F}(1,14)=6.74, \mathrm{p}<.05)$. Post hoc analysis found that the 6-OHDA+Vehicle treated animals had significantly less $\mathrm{p}-\mathrm{CaMKII} \alpha(\mathrm{Thr} 286)$ compared to all other treatment groups $(\mathrm{p}<.05)$. No significant difference was found between 6-OHDA+CTEP treated animals and Sham+Vehicle or Sham+CTEP treated controls. This western blot was visualized using IR800 fluorophore on Licor Odyssey FC equipment, normalized total protein signal following a Fast Green Stain. 

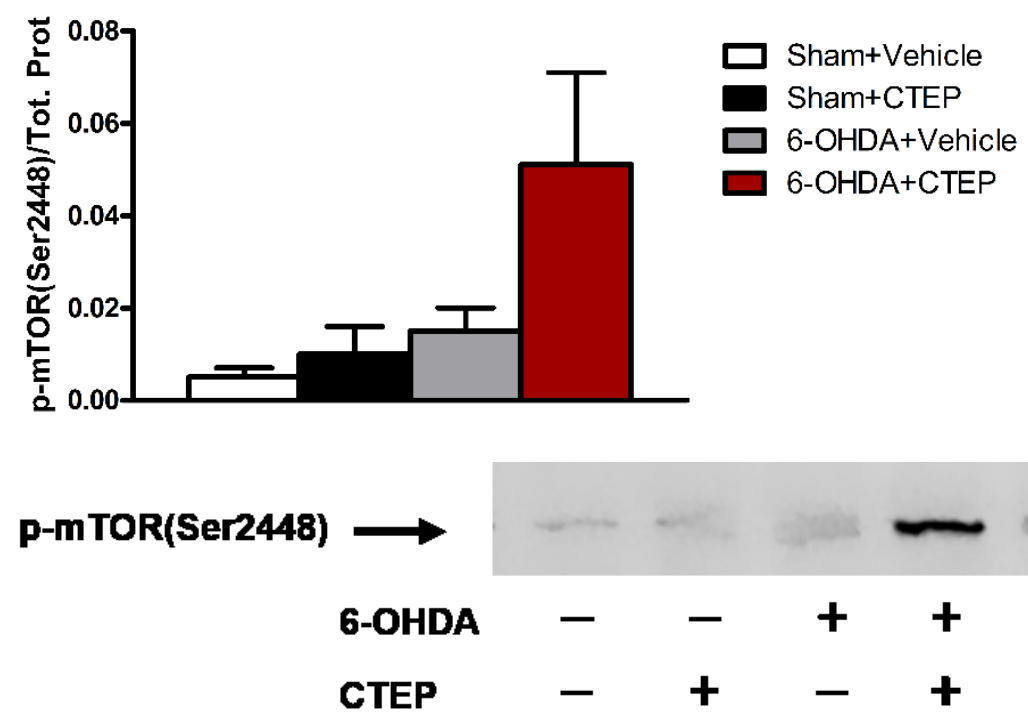

Figure 13 Striatal p-mTOR(Ser2448) (1 week CTEP)

Striatal p-mTOR(Ser2448) was measured after 1 week of CTEP administration by western blot. A two-way ANOVA found a significant effect of 6-OHDA $(\mathrm{F}(1,12)=5.49, \mathrm{p}<.05)$, however no interaction effect was found. Despite this, it is clear the elevation is confined to 6$\mathrm{OHDA}+\mathrm{CTEP}$ treated mice, having barely missed significance $(\mathrm{F}(1,12)$ $=3.57, \mathrm{p}=.08)$. This western blot was visualized using IR800 fluorophore on Licor Odyssey FC equipment, normalized total protein signal following a Fast Green Stain. 

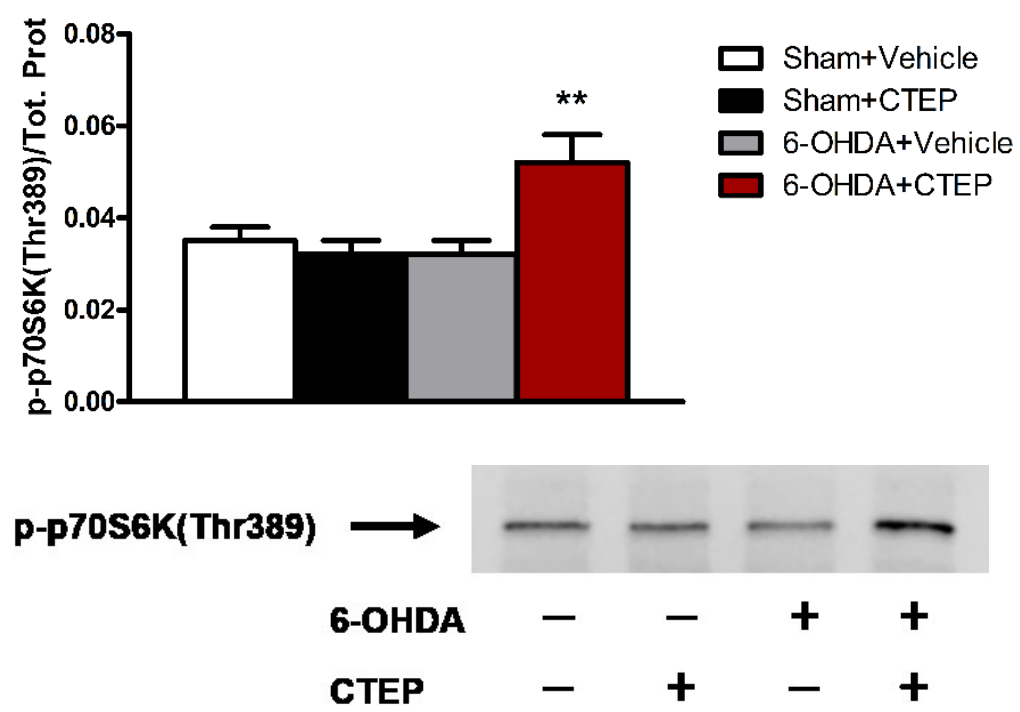

Figure 14 Striatal p-p70S6K(Thr389) (1 week CTEP)

Striatal p-p70S6K(Thr389) was measured after 1 week of CTEP administration by western blot. A two-way ANOVA found a significant CTEP x 6-OHDA interaction effect $(F(1,12)=9.14, p<.05)$. Post hoc analysis found that 6-OHDA+CTEP treated animals had significantly more p-p70S6K(Thr389) levels compared to all other treatment groups $(\mathrm{p}<.01)$. This western blot was visualized using IR800 fluorophore on Licor Odyssey FC equipment, normalized total protein signal following a Fast Green Stain. 


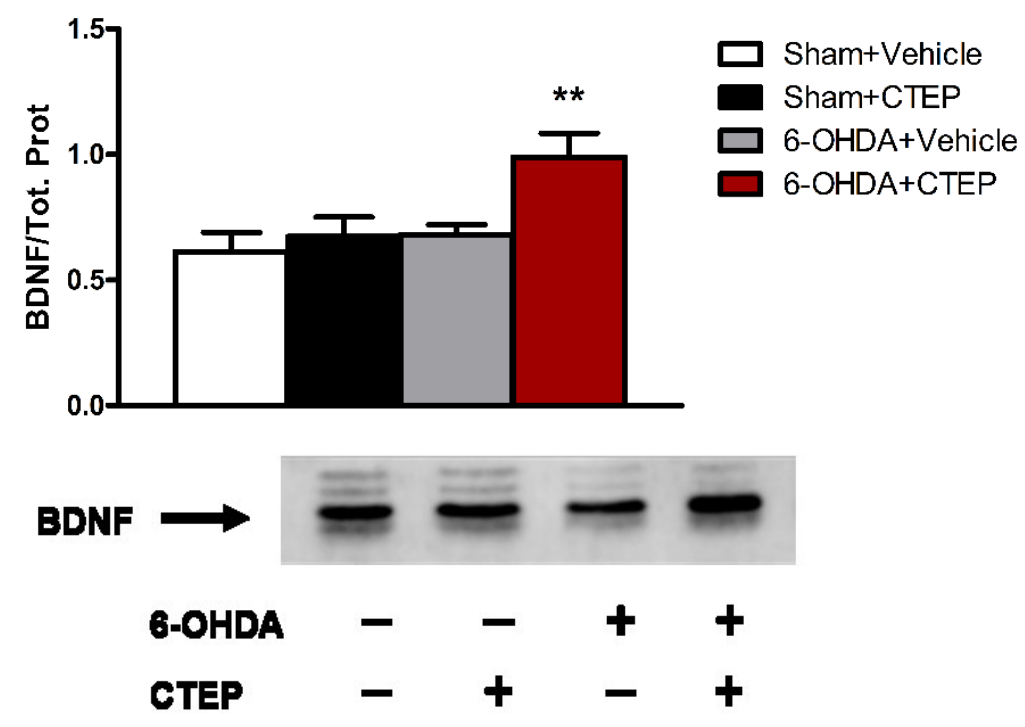

Figure 15 Striatal BDNF (1 week CTEP)

Striatal BDNF was measured after 1 week of CTEP administration by western blot. A two-way ANOVA found a significant 6-OHDA $(\mathrm{F}(1,12)$ $=6.61, \mathrm{p}<.05)$ and CTEP effects $(\mathrm{F}(1,12)=6.15, \mathrm{p}<.05)$, however no CTEP x 6-OHDA interaction was found $(\mathrm{F}(1,12)=2.78, \mathrm{p}=.12)$. Post hoc analysis found that 6-OHDA+CTEP treated animals had significantly more BDNF levels compared to all other treatment groups $(\mathrm{p}<.01)$. This western blot was visualized using IR800 fluorophore on Licor Odyssey FC equipment, normalized total protein signal following a Fast Green Stain. 

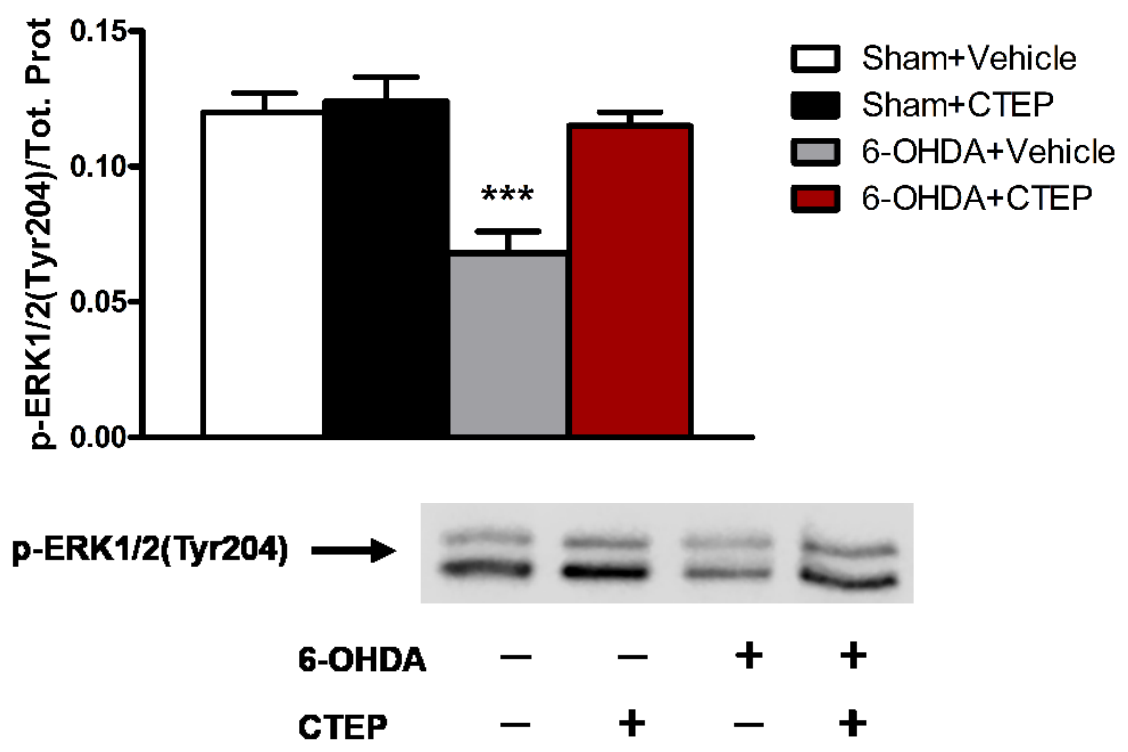

Figure 16 Motor Cortex p-ERK1/2(Tyr204) (1 week CTEP)

p-ERK1/2(Tyr204) was measured in the motor cortex by western blot after 1 week of CTEP administration. A two way ANOVA found a significant CTEP x 6-OHDA interaction $(F(1,14)=7.88, p<.05)$. Post hoc analysis found that 6-OHDA+Vehicle treated animals had significantly less p-ERK1/2(Tyr204) compared to all other treatment groups $(\mathrm{p}<.001)$. Importantly, 6-OHDA+CTEP treated animals were not significantly different from Sham controls. This western blot was visualized using IR800 fluorophore on Licor Odyssey FC equipment, normalized total protein signal following a Fast Green Stain. 

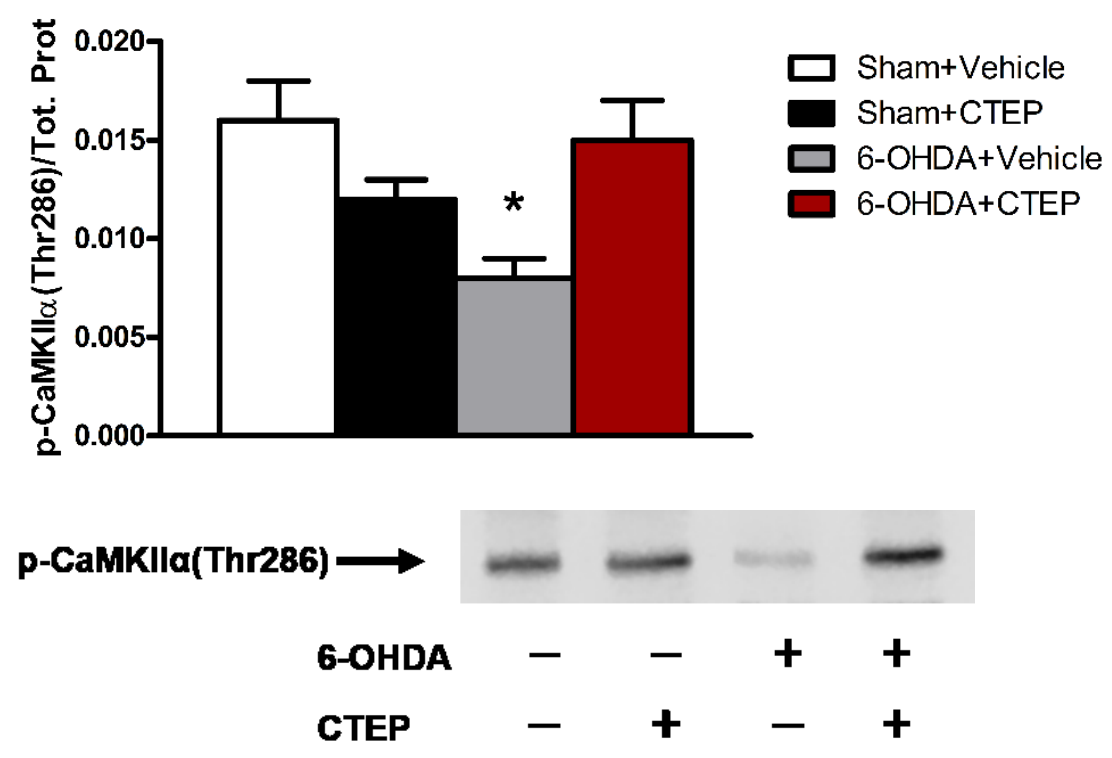

Figure 17 Motor Cortex p-CaMKII $\alpha($ Thr286) (1 week CTEP)

p-CaMKII $\alpha(T h r 286)$ was measured in the motor cortex by western blot after 1 week of CTEP administration. A two-way ANOVA found a significant CTEP x 6-OHDA interaction effect $(\mathrm{F}(1,14)=17.99, \mathrm{p}<$ .001). Post hoc analysis found that the 6-OHDA+Vehicle treated animals had significantly less p-CaMKII $\alpha$ (Thr286) compared to all other treatment groups $(\mathrm{p}<.05)$. No significant difference was found between 6-OHDA+CTEP treated animals and Sham+Vehicle or Sham + CTEP treated controls. This western blot was visualized using IR800 fluorophore on Licor Odyssey FC equipment, normalized total protein signal following a Fast Green Stain. 


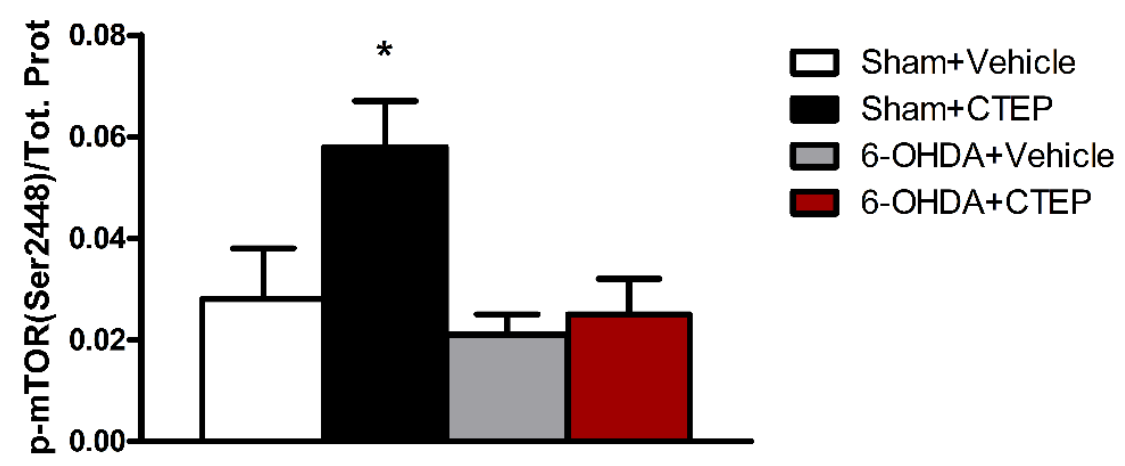

P-mTOR(Ser2448)

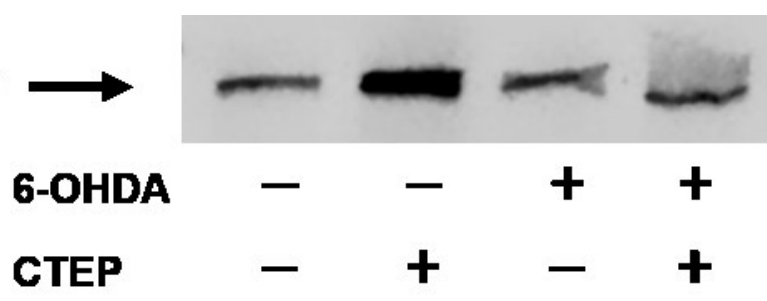

Figure 18 Motor Cortex p-mTOR(Ser2448) (1 week CTEP)

p-mTOR(Ser2448) was measured in the motor cortex by western blot after 1 week of CTEP administration. A two-way ANOVA found a significant 6-OHDA $(\mathrm{F}(1,12)=6.109, \mathrm{p}=.0294)$ and CTEP effects $(\mathrm{F}(1,12)=4.803, \mathrm{p}=.0489)$, however no interaction effect was found. Post hoc analysis found that Sham+CTEP treated animals had significantly more p-mTOR (Ser2448) levels compared to all other treatment groups $(\mathrm{p}<.05)$. This western blot was visualized using IR800 fluorophore on Licor Odyssey FC equipment, normalized total protein signal following a Fast Green Stain. 


\section{Weeks of CTEP Administration: Behaviour}

In this second experiment we wished to determine any long term effects of CTEP treatment. As such, following a 6-OHDA induced lesion CTEP was given over a 12 week period via a chocolate pudding suspension. Following 6-OHDA treatment (30 days later) but prior to starting CTEP administration, all animals were challenged with apomorphine in order to ensure that the toxicant induced a robust parkinsonian state. Indeed, the ANOVA found a significant main effect at Day 30 for 6-OHDA lesion induced apomorphine rotations $(\mathrm{F}(2,31)$ $=11.64, \mathrm{p}<.001)$. In fact, all 6-OHDA treated animals demonstrated significant apomorphine induced rotations $(\mathrm{p}<.01)$ (Figure 19). Near the end of the experiment, as CTEP was still being administered, all animals were tested in a modified reversal Morris water maze to measure cognitive flexibility. After analyzing the time to find the platform during probe trials, a one way ANOVA found a significant difference between the treatment groups $(\mathrm{F}(2,27)=4.33, \mathrm{p}<.05)$ (Figure 20). As shown in Figure 20 and confirmed by the follow up comparisons, 6-OHDA lesion induced a deficit in water maze performance, as indicated by an increased time to find the hidden 
platform $(\mathrm{p}<.05)$. However, this deficit was ameliorated by CTEP treatment. On the last day of the experiment - after 12 weeks of CTEP treatment - the mice were tested on an accelerating rota rod where a clear significant difference between the groups was evident $(F(2,27)=$ $10.79, \mathrm{p}<.001$ ) (Figure 21). The comparison revealed that the 6-OHDA lesion was associated with deficits on the rota rod, such that these animals fell off sooner $(\mathrm{p}<.01)$, and once again CTEP reversed this effect. Following the rota rod test, animals were tested once again for apomorphine induced rotations. A one-way ANOVA found a significant overall effect $(F(2,27)=69.59, \mathrm{p}<.001)($ Figure 19). The follow up analysis revealed that the 6-OHDA lesion provoked a continued marked elevation of apomorphine induced rotations $(\mathrm{p}<.001)$, but CTEP completely reversed this effect. 


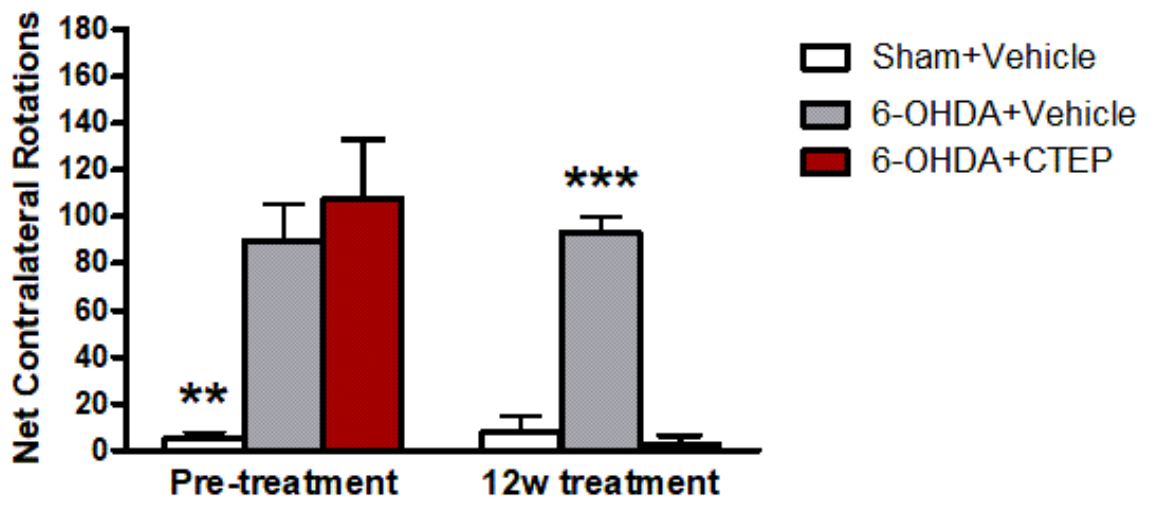

Figure 19 Apomorphine Induced Rotations (12 week CTEP)

Animals were given injections of apomorphine 30 days after 6-OHDA administration. The net contra-lateral rotations over a 20 minute period were recorded. A one-way ANOVA found a significant main effect at day $30(\mathrm{~F}(2,31)=11.64, \mathrm{p}<.001)$. After 12 weeks of CTEP administration a one-way ANOVA found a significant effect $(\mathrm{F}(2,27)=$ $69.59, \mathrm{p}<.001)$. Post hoc analysis found that 6 -OHDA+Vehicle was significantly different from all other treatment groups $(p<.001)$, while there was no statistical difference between the Sham+Vehicle and Sham + CTEP groups. 


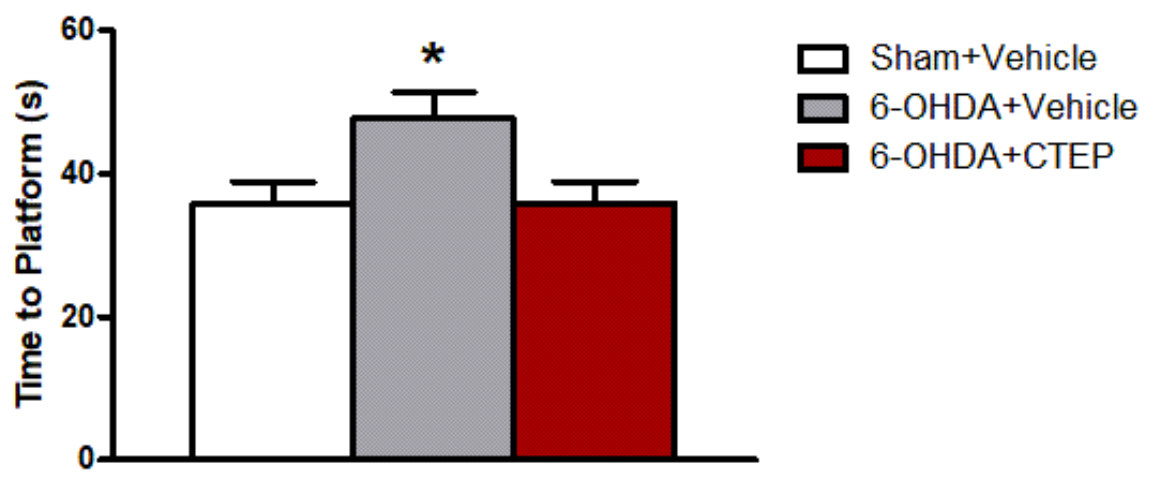

Figure 20 Reversal water maze (12 week CTEP)

Animals were tested for cognitive flexibility on the reversal Morris water maze after 12 weeks of CTEP administration. A one way ANOVA found a significant treatment effect $(\mathrm{F}(2,27)=4.33, \mathrm{p}<.05)$. Post hoc analysis found a significant difference between the 6-OHDA+Vehicle treatment group and all other treatment groups $(p<.05)$. 


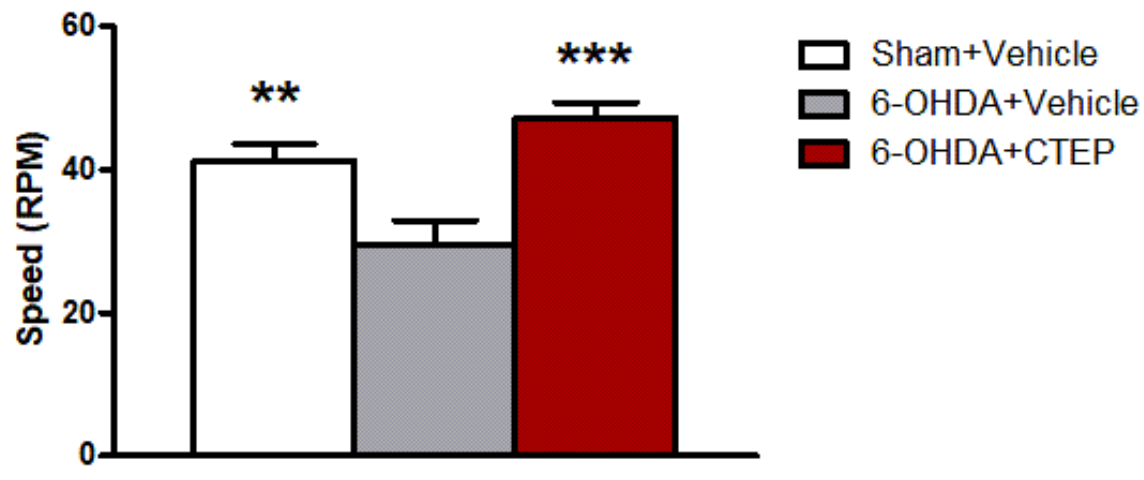

Figure 21 Rota Rod (12 week CTEP)

Animals were tested on the accelerating rota rod after 12 weeks of CTEP administration. A one way ANOVA found a significant treatment effect $(\mathrm{F}(2,27)=10.79, \mathrm{p}<.001)$. Post hoc analysis found a significant difference between the 6-OHDA+Vehicle treatment group and all other treatment groups $(* * \mathrm{p}<.01, * * * \mathrm{p}<.001)$. 


\section{Weeks of CTEP Administration: Histology}

Striatal $\mathrm{TH}+$ terminal density was once again measured to assess the striatal dopaminergic lesion. Paralleling the behavioral data, striatal $\mathrm{TH}+$ terminal density was significantly different between the treatment

groups $(\mathrm{F}(2,12)=26.57, \mathrm{p}<.001)$ (Figure 22). The statistical comparisons indicated that 6-OHDA did indeed reduce the density of striatal TH+ fibre staining $(\mathrm{p}<.01)$ and that CTEP reversed this decline. Similarly, the number of $\mathrm{TH}+\mathrm{SNc}$ dopamine neuronal cell bodies also varied among the treatment groups $(\mathrm{F}(2,15)=17.41, \mathrm{p}<.001)$ (Figure 23). Indeed, as expected, 6-OHDA provoked a loss of $\mathrm{SNc} \mathrm{TH}+$ neurons $(\mathrm{p}<.0001)$, however despite the marked changes in the striatum CTEP had no effect on $\mathrm{TH}+$ neuronal counts in the SNc.

Our data suggested that CTEP may be promoting striatal recovery through increase DA innervation. As an index of postsynaptic activity to determine functionality of these fibres, the number of FosB+ striatal cells were assessed. In this regard, the ANOVA found a significant difference between the treatment groups $(\mathrm{F}(2,12)=6.85, \mathrm{p}<$ .05) (Figure 24). Consistent with its impact on striatal dopaminergic 
terminal density, 6-OHDA also elevated the number of striatal FosB+ cells $(\mathrm{p}<.05)$ which was reversed following CTEP treatment. Similarly, the ANOVA found a significant effect within the motor cortex $(F(2,12)$ $=6.61, \mathrm{p}<.05)$ in the number of FosB + cells (Figure 25). Once again, the number FosB + cortical cells were increased by 6 -OHDA $(\mathrm{p}<.05)$ and this effect was rescued by CTEP. 


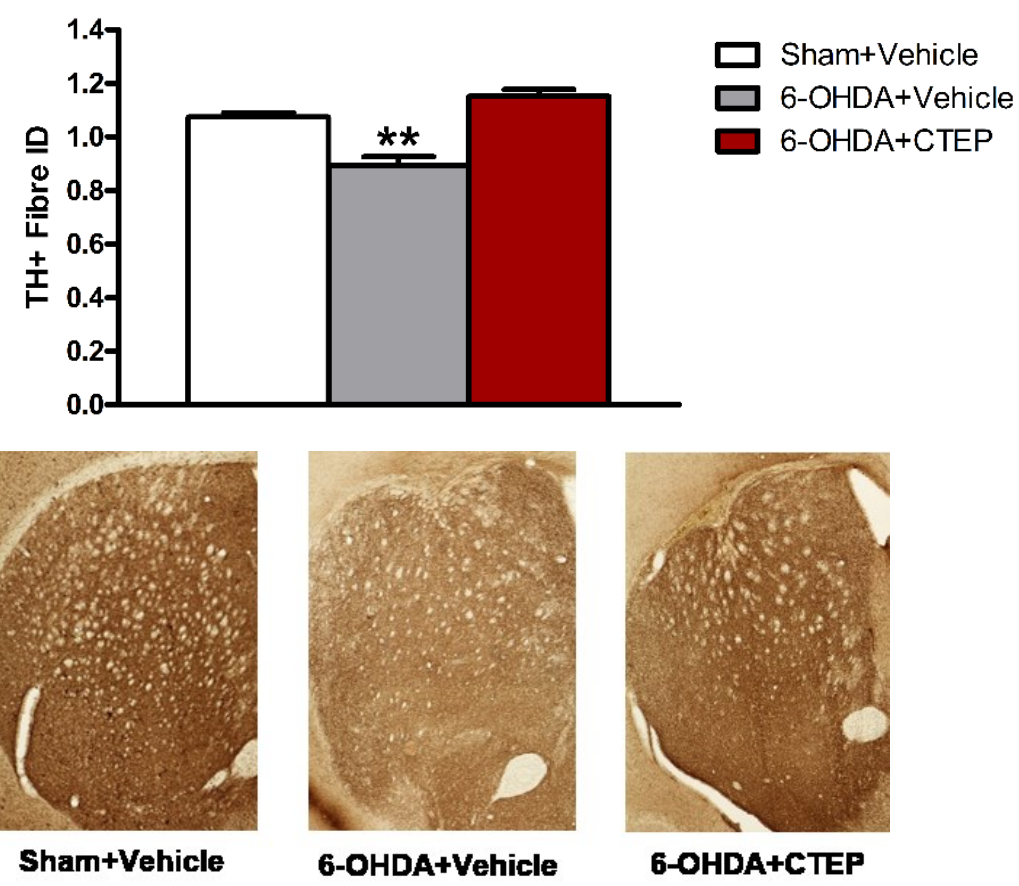

Figure 22 Striatal TH+ Histology (12 week CTEP)

The integrated density of striatal sections immunolabelled with TH was measured using ImageJ software after 12 weeks of CTEP administration. A one way ANOVA found a significant main effect $(\mathrm{F}(2,12)=26.57, \mathrm{p}<.001)$. Post hoc analysis found that the 6OHDA+Vehicle treatment group had significantly less $\mathrm{TH}+$ staining compared to all other treatment groups $(p<.01)$. There was no statistical difference between the 6-OHDA+CTEP and Sham+Vehicle treated animals. 

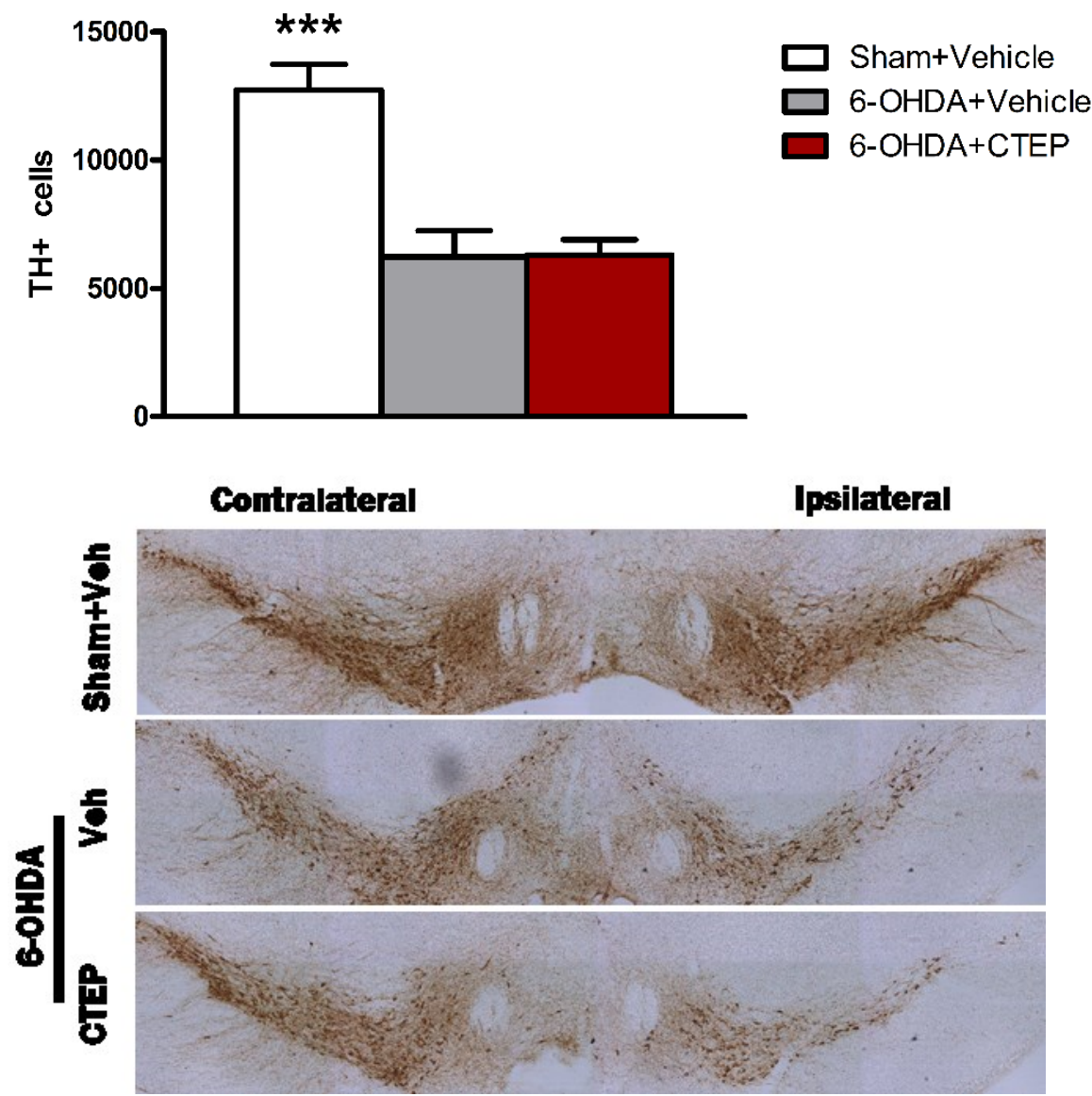

Figure 23 Nigral TH+ cell counts (12 week CTEP)

A stereological count of $\mathrm{TH}+$ cells in the substantia nigra was complete after 12 weeks of CTEP administration. A one way ANOVA found a significant main effect $(\mathrm{F}(2,15)=17.41, \mathrm{p}<.001)$. Post hoc analysis found that both 6-OHDA+Vehicle and 6-OHDA+CTEP treated animals had significantly less TH+ cells compared to Sham+Vehicle controls ( $p$ $<.0001$ ), and the CTEP administration had no effect on nigral $\mathrm{TH}+$ cell counts. 


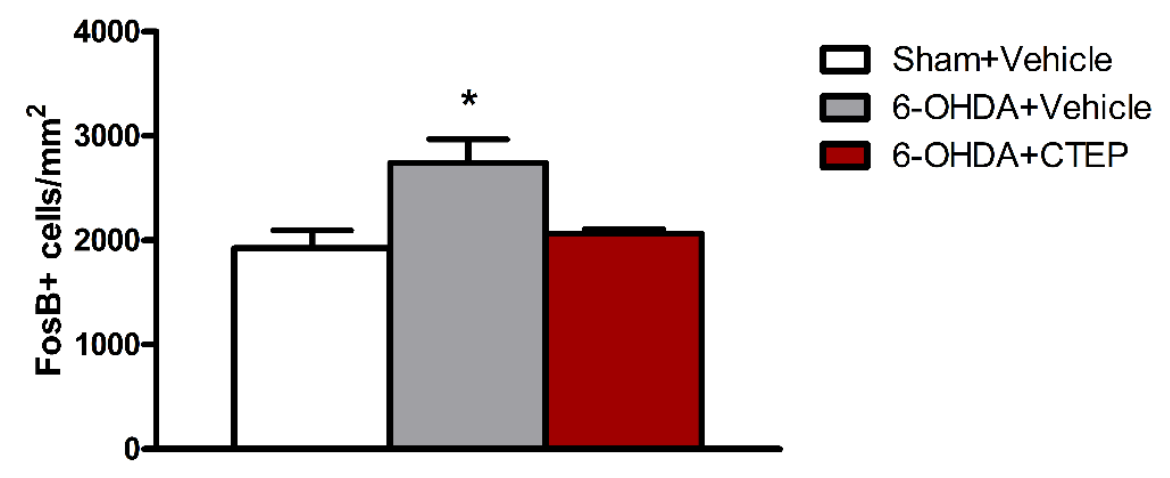

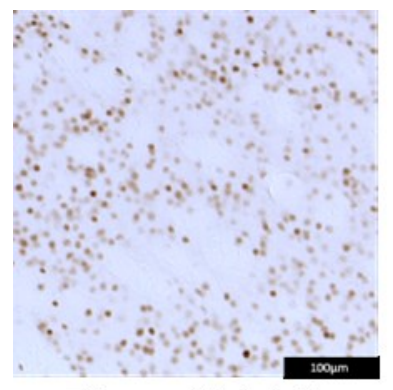

Sham+Vehicle

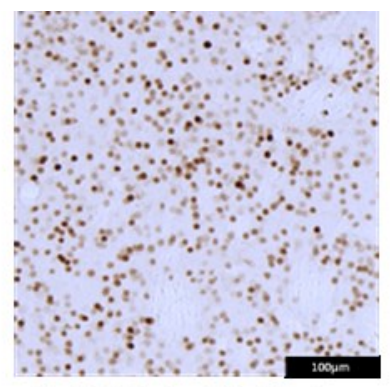

6-OHDA+Vehlcle

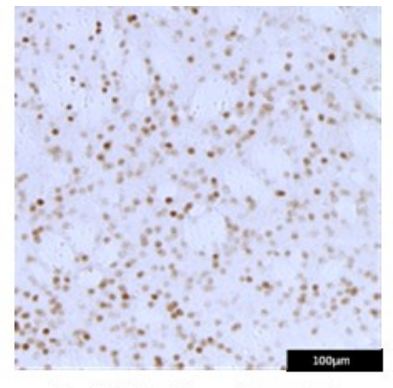

6-OHDA+CTEP

Figure 24 Striatal FosB+ cells (12 week CTEP)

An automated cell count of FosB + cells in the striatum was completed using ImageJ software after 12 weeks of CTEP administration. A one way ANOVA found a significant main effect $(F(2,12)=6.85, \mathrm{p}<.05)$. Post hoc analysis found that 6-OHDA+Vehicle treated animals had significantly more FosB + cells compared to both Sham+Vehicle and 6OHDA+CTEP treated animalsv $(\mathrm{p}<.05)$. No significant difference was found between 6-OHDA+CTEP treated animals and Sham+Vehicle controls. 


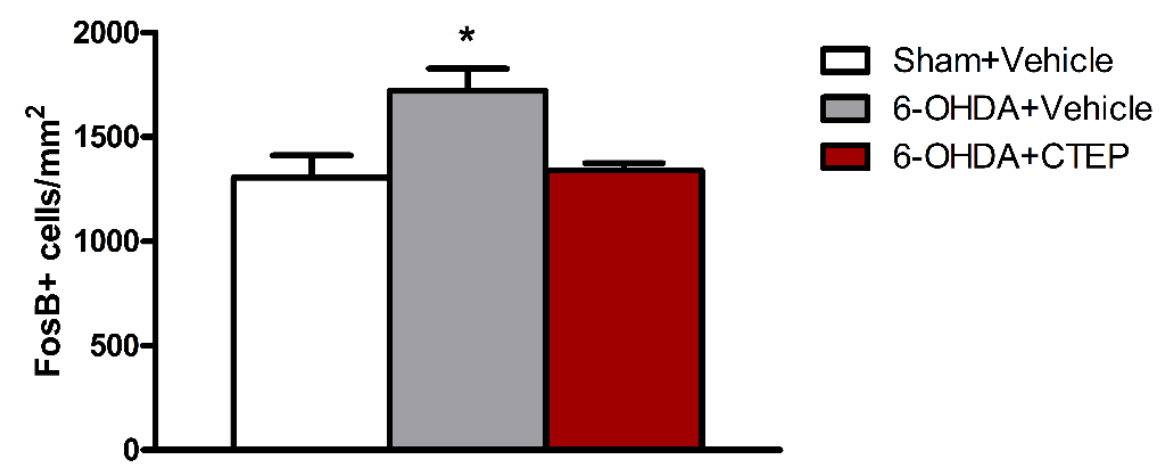

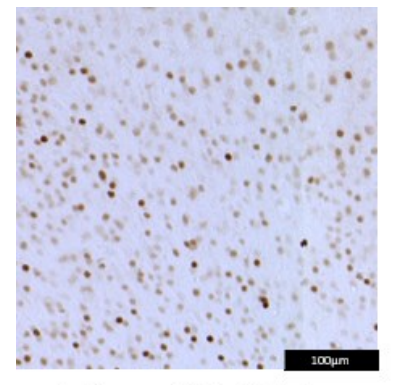

Sham+Vehicle

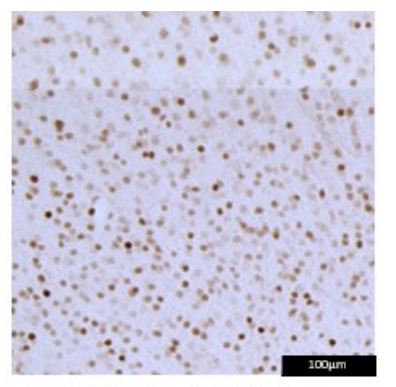

6-OHDA+Vehicle

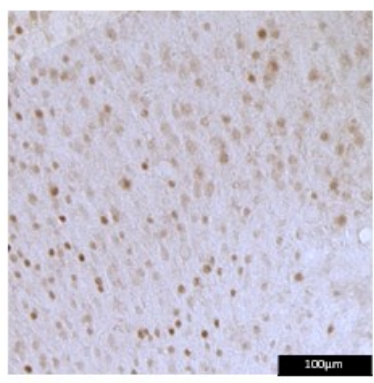

6-OHDA+CTEP

Figure 25 Motor Cortex FosB + cells (12 week CTEP)

An automated cell count of FosB + cells in the motor cortex was completed using ImageJ software after 12 weeks of CTEP administration. A one way ANOVA found a significant main effect $(\mathrm{F}(2,12)=6.61, \mathrm{p}<.05)$. Post hoc analysis found that $6-\mathrm{OHDA}+\mathrm{Vehicle}$ treated animals had significantly more FosB + cells compared to both Sham+Vehicle and 6-OHDA+CTEP treated animals $(\mathrm{p}<.05)$. No significant difference was found between 6-OHDA+CTEP treated animals and Sham + Vehicle controls. 


\section{Weeks of CTEP Administration: Molecular}

Following 12 weeks of CTEP administration the downstream signaling partners of mGluR5 were measured using Western blot. Protein levels of p-ERK1/2(Tyr204) and p-CaMKII $\alpha($ Thr286) within the striatum were not significantly different between the groups $(F(2,12)$ $=0.24, \mathrm{p}>.05$ and $\mathrm{F}(2,11)=.034, \mathrm{p}>.05)$, respectively (Figure 27). However, there was a significant difference between the groups with respect to $p-m T O R(\operatorname{Ser} 2448)$ levels in the striatum $(F(2,9)=20.19, p<$ .001) (Figure 28). Indeed, CTEP increased striatal p-mTOR(Ser2448) in 6-OHDA lesioned mice, relative to those that did not received the drug $(\mathrm{p}<.01)$. Similarly when comparing levels of the downstream effector of mTOR, p-p70S6K(Thr389), the ANOVA also revealed a significant effect $(\mathrm{F}(2,8)=5.97, \mathrm{p}<.05)$ (Figure 29). Identical to the $\mathrm{p}$ mTOR(Ser2448) data, p-p70S6K(Thr389) was elevated by CTEP in the context of the 6-OHDA lesion $(\mathrm{p}<.05)$. Finally, an ANOVA revealed a significant effect for striatal BDNF levels $(\mathrm{F}(2,9)=7.33, \mathrm{p}<.05)$ (Figure 30). Accordingly, BDNF was up-regulated by the CTEP regimen in the context of the 6-OHDA lesion $(\mathrm{p}<.05)$. 
Interestingly, following 12 weeks of CTEP the pattern of protein signalling changes within the motor cortex were somewhat different than what was observed in the striatum. For instance, unlike the striatum, p-mTOR(Ser2448) was not significantly changed within the motor cortex $\mathrm{F}(2,9)=.01, \mathrm{p}>.05)($ Figure 31). However, cortical pERK1/2(Tyr204) levels were affected by the treatments $(F(2,12)=3.90$, $\mathrm{p}<.05)$ (Figure 32). Indeed, CTEP elevated p-ERK1/2(Tyr204) levels in the 6-OHDA lesioned mice $(\mathrm{p}<.05)$. Similarly, $\mathrm{p}$-CaMKII $\alpha($ Thr286) was affected by the treatments $(\mathrm{F}(2,12)=6.23, \mathrm{p}<.05$ (Figure 33). But in this case, 6-OHDA reduced p-CaMKII $\alpha($ Thr286) levels $(\mathrm{p}<.05)$ and this effect was reversed by CTEP. 


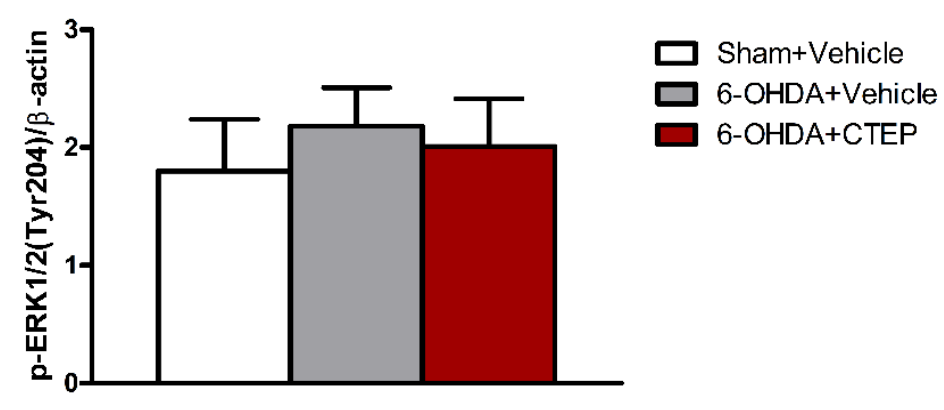

P-ERK1/2(Tyr204)
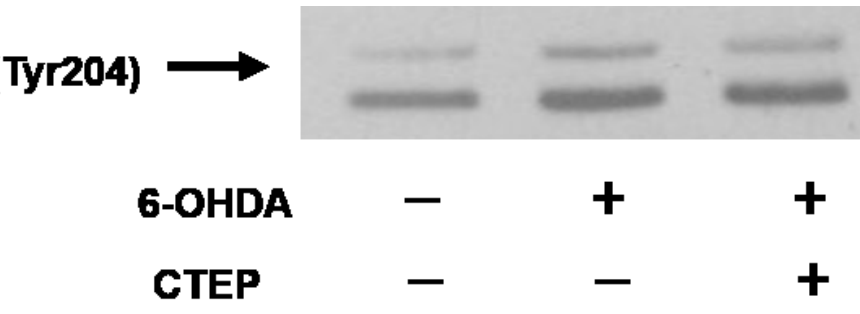

B-actin
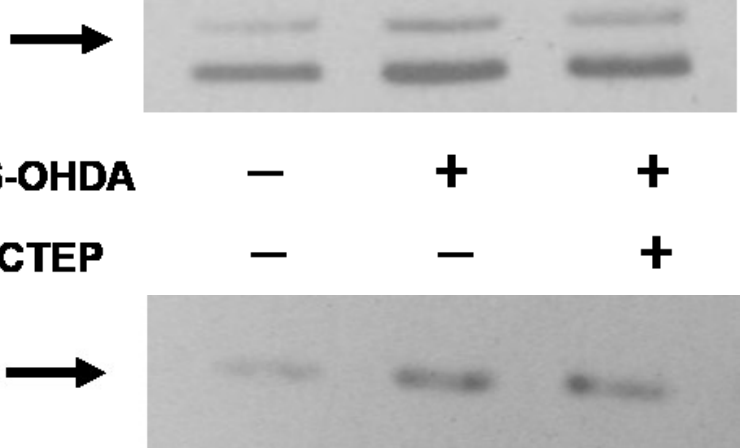

Figure 26 Striatal p-ERK1/2(Tyr204) (12 week CTEP)

Striatal p-ERK1/2(Tyr204) was measured after 12 weeks of CTEP administration by western blot. A one way ANOVA found no main effects, or interactions $(F(2,12)=0.24, p>.05)$. This western blot was visualized following ECL chemiluminescent reaction and $\mathrm{x}$-ray film exposure. Protein signal was normalized to $\beta$-actin also detected by chemiluminescence and x-ray film. 

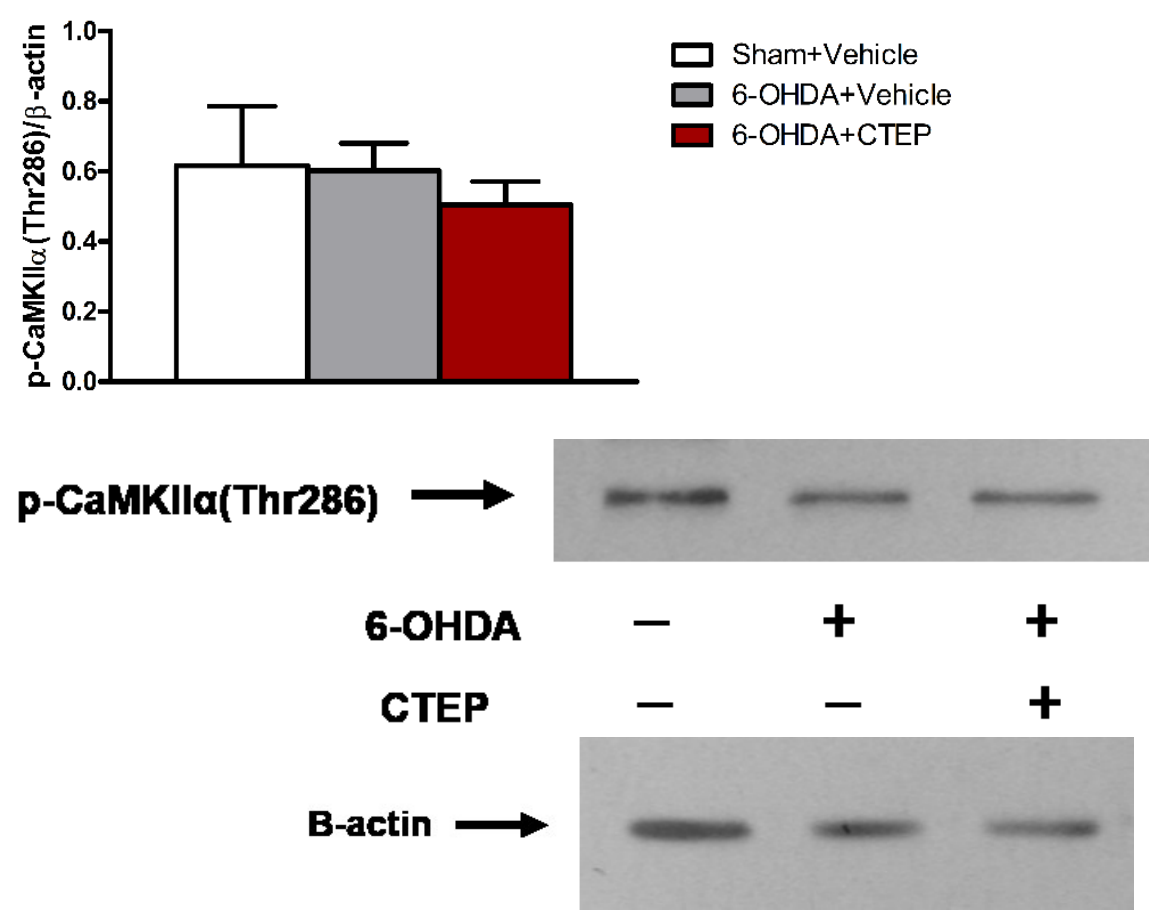

Figure 27 Striatal p-CaMKII $\alpha($ Thr286) (12 week CTEP)

Striatal p-CAMKII $\alpha($ Thr286) was measured after 12 weeks of CTEP administration by western blot. A one way ANOVA found no main effects, or interactions $(\mathrm{F}(2,11)=0.34, \mathrm{p}>.05)$. This western blot was visualized following ECL chemiluminescent reaction and x-ray film exposure. Protein signal was normalized to $\beta$-actin also detected by chemiluminescence and x-ray film. 

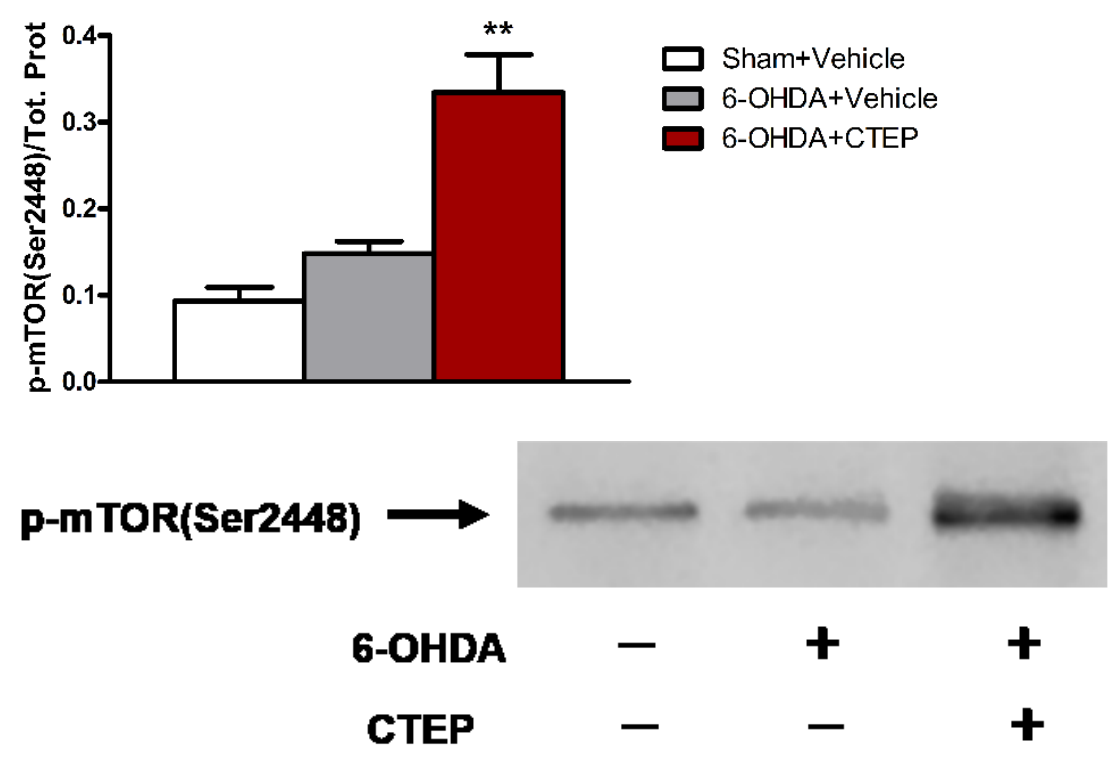

Figure 28 Striatal p-mTOR(Ser2448) (12 week CTEP)

Striatal p-mTOR(Ser2448) was measured after 12 weeks of CTEP administration by western blot. A one way ANOVA found a significant main effect $(\mathrm{F}(2,9)=20.19, \mathrm{p}<.001)$. Post hoc analysis found that 6 OHDA+CTEP treated animals had significantly more $p$ mTOR(Ser2448) levels compared to all other treatment groups $(\mathrm{p}<.01)$. This western blot was visualized using IR800 fluorophore on Licor Odyssey FC equipment, normalized total protein signal following a Fast Green Stain. 

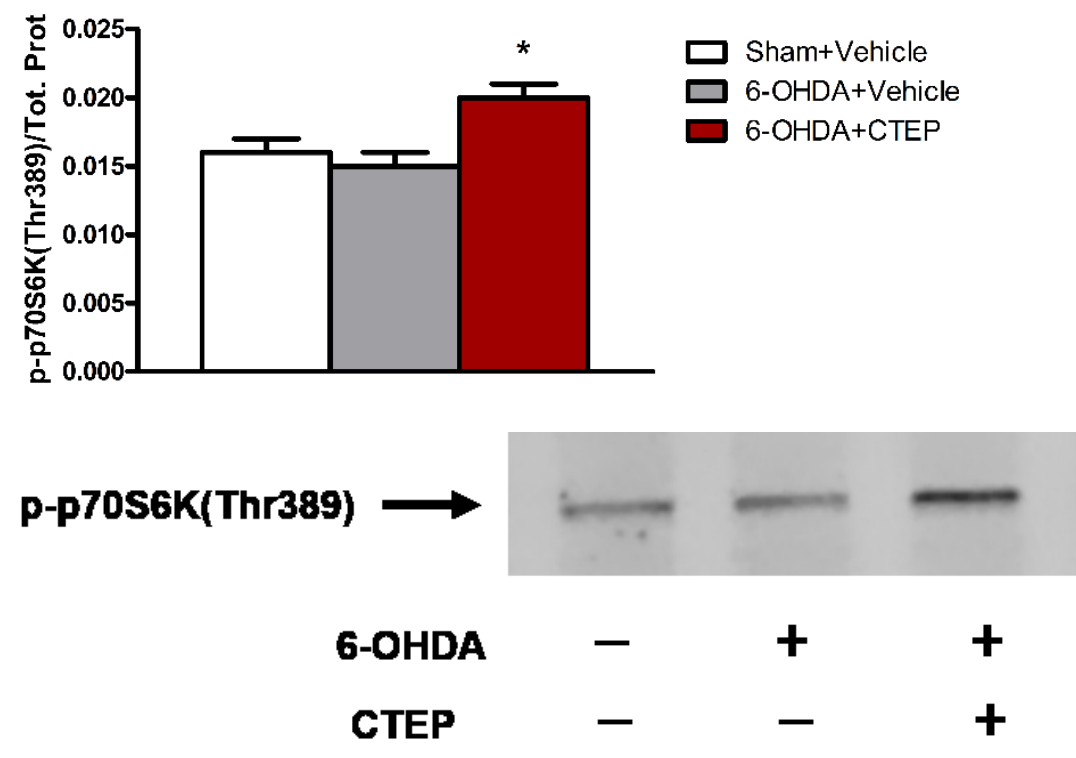

Figure 29 Striatal p-p70S6K(Thr389) (12 week CTEP)

Striatal p-p70S6K(Thr389) was measured after 12 weeks of CTEP administration by western blot. A one way ANOVA found a significant main effect $(\mathrm{F}(2,8)=5.97, \mathrm{p}<.05)$. Post hoc analysis found that 6 OHDA+CTEP treated animals had significantly more pp70S6K(Thr389) levels compared to all other treatment groups ( $\mathrm{p}<$ .05). This western blot was visualized using IR800 fluorophore on Licor Odyssey FC equipment, normalized total protein signal following a Fast Green Stain. 


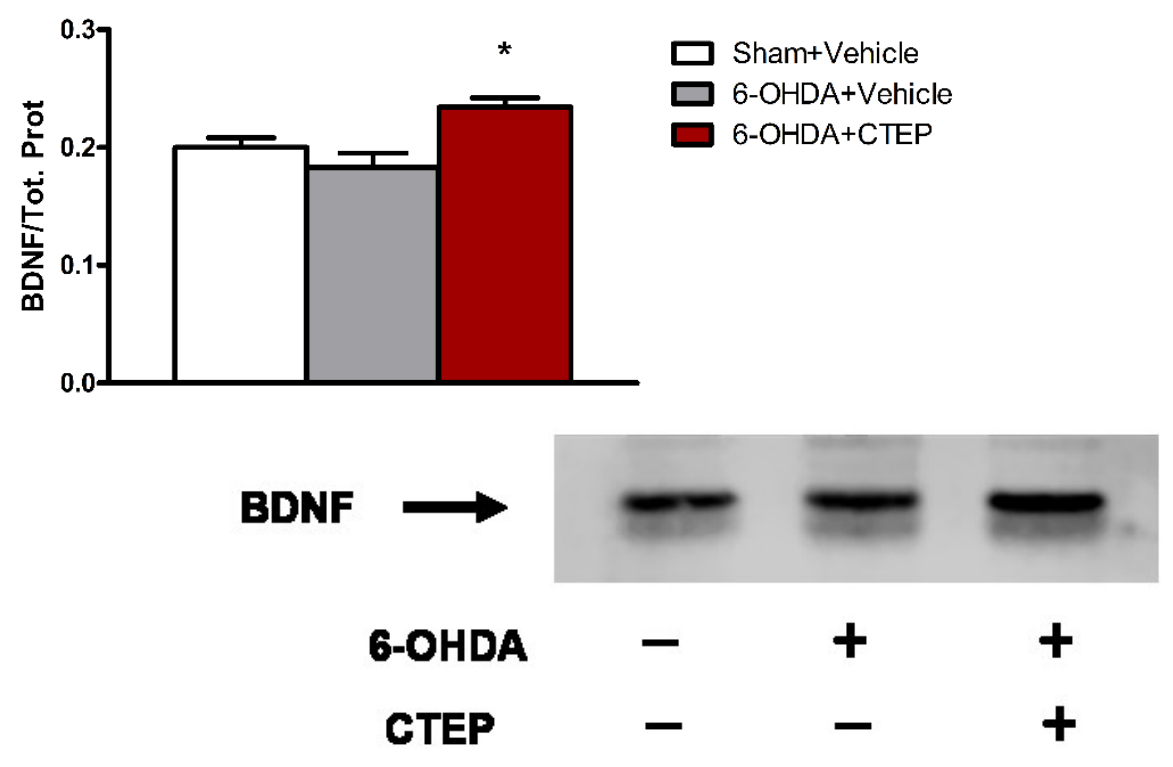

Figure 30 Striatal BDNF (12 week CTEP)

Striatal BDNF was measured after 12 weeks of CTEP administration by western blot. A one way ANOVA found a significant main effect $(F(2,9)$ $=7.33, \mathrm{p}<.05)$. Post hoc analysis found that 6 -OHDA+CTEP treated animals had significantly more BDNF levels compared to all other treatment groups $(\mathrm{p}<.05)$. This western blot was visualized using IR800 fluorophore on Licor Odyssey FC equipment, normalized total protein signal following a Fast Green Stain. 

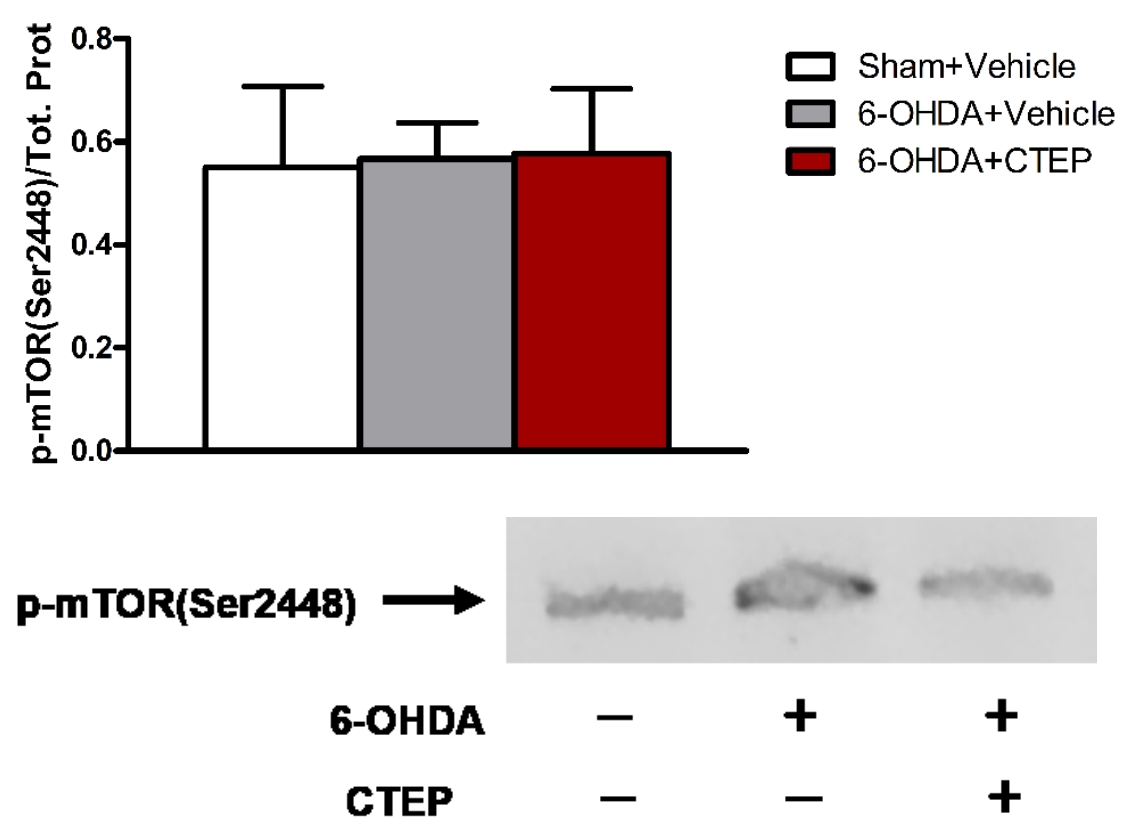

Figure 31 Motor Cortex p-mTOR(Ser2448) (12 week CTEP)

p-mTOR(Ser2448) was measured in the motor cortex by western blot after 12 weeks of CTEP administration. A one way ANOVA found no significant effects $(\mathrm{F}(2,9)=.01, \mathrm{p}>.05)$. This western blot was visualized using IR800 fluorophore on Licor Odyssey FC equipment, normalized total protein signal following a Fast Green Stain. 

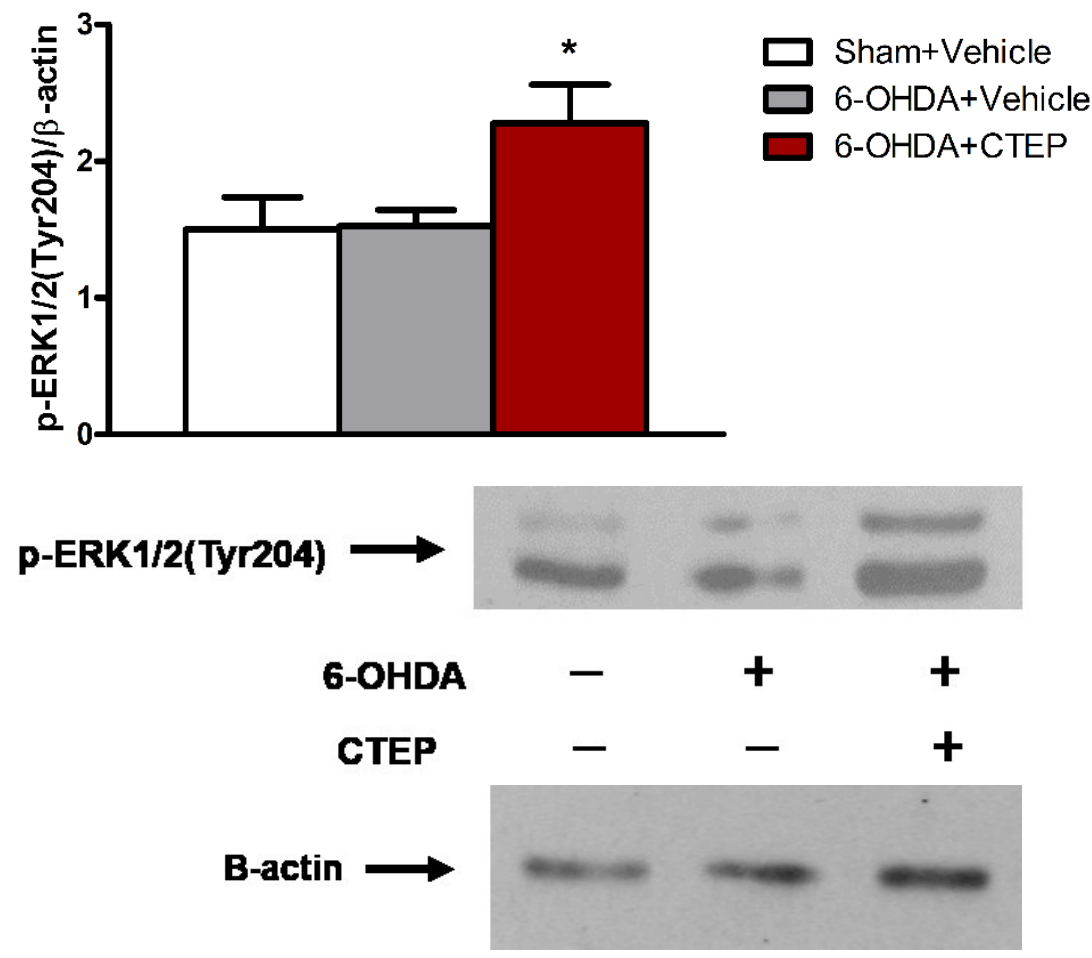

Figure 32 Motor Cortex p-ERK1/2(Tyr204) (12 week CTEP)

p-ERK1/2(Tyr204) was measured in the motor cortex by western blot after 12 weeks of CTEP administration. A one way ANOVA found significant main effect $(F(2,12)=3.90, p<.05)$. Post hoc analysis found that 6-OHDA+CTEP treated animals had significantly more pERK1/2(Tyr204) compared to all other treatment groups $(\mathrm{p}<.05)$. This western blot was visualized following ECL chemiluminescent reaction and $x$-ray film exposure. Protein signal was normalized to $\beta$-actin also detected by chemiluminescence and x-ray film. 


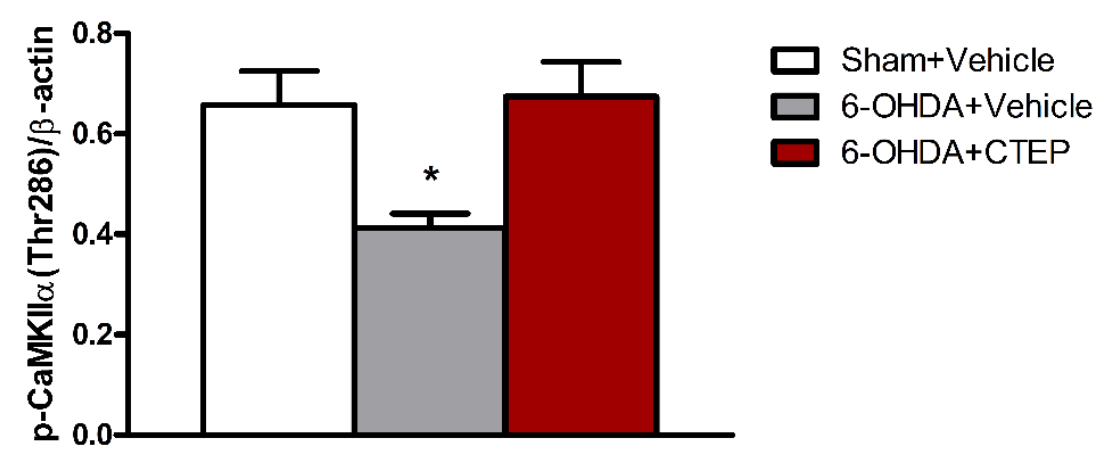

p-CaMKIla(Thr286)

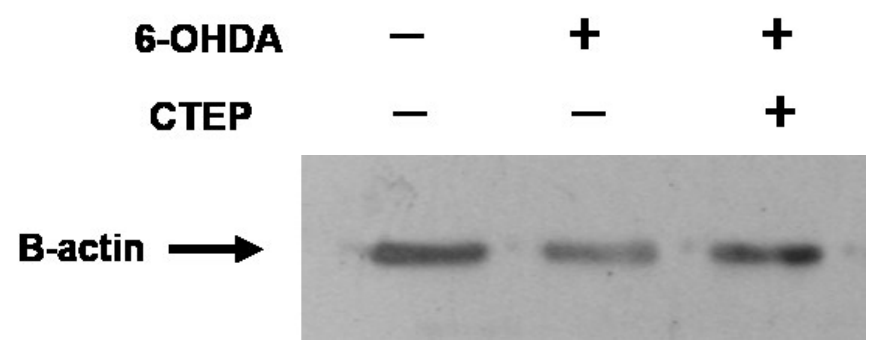

\section{Figure 33 Motor Cortex p-CaMKII $\alpha($ Thr286) (12 week CTEP)}

p-CaMKII $\alpha($ Thr286) was measured in the motor cortex by western blot after 12 weeks of CTEP administration. A one way ANOVA found a significant main effect $(\mathrm{F}(2,12)=6.23, \mathrm{p}<.05)$. Post hoc analysis found that the 6-OHDA+Vehicle treated animals had significantly less pCaMKII $\alpha($ Thr286) compared to all other treatment groups $(\mathrm{p}<.05)$. No significant difference was found between 6-OHDA+CTEP treated animals and Sham + Vehicle treated controls. This western blot was visualized following ECL chemiluminescent reaction and $\mathrm{x}$-ray film exposure. Protein signal was normalized to $\beta$-actin also detected by chemiluminescence and x-ray film. 


\section{Effects of CTEP following discontinuation of treatment}

It was of interest to assess whether CTEP might have enduring effects that persist beyond its administration time. Hence, one experimental cohort of animals received 1 week of CTEP, followed by a 1 week drug washout period. Although the repeated measures ANOVA failed to reach significance, there was a main effect between the treatment groups $F(2,21)=22.05, \mathrm{p}<.01$. (Figure 34$)$. The follow up comparison revealed that CTEP produced it expected reduction in rotations after 1 week of treatment $(\mathrm{p}<.05)$; however, following the 1 week washout there was no longer any significant effect $(p>.05)$.

The integrated density of $\mathrm{TH}+$ fibres in the striatum was also compared and the one way ANOVA found a significant main effect $(\mathrm{F}(2,11)=12.72, \mathrm{p}<.01)($ Figure 35$)$. Specifically, 6-OHDA induced the expected marked reduction in striatal $\mathrm{TH}+$ terminal density $(\mathrm{p}<$ 0.05), however, CTEP treatment once again reversed this effect such that density was not significantly different from sham controls, despite the observed behavioural regression. 


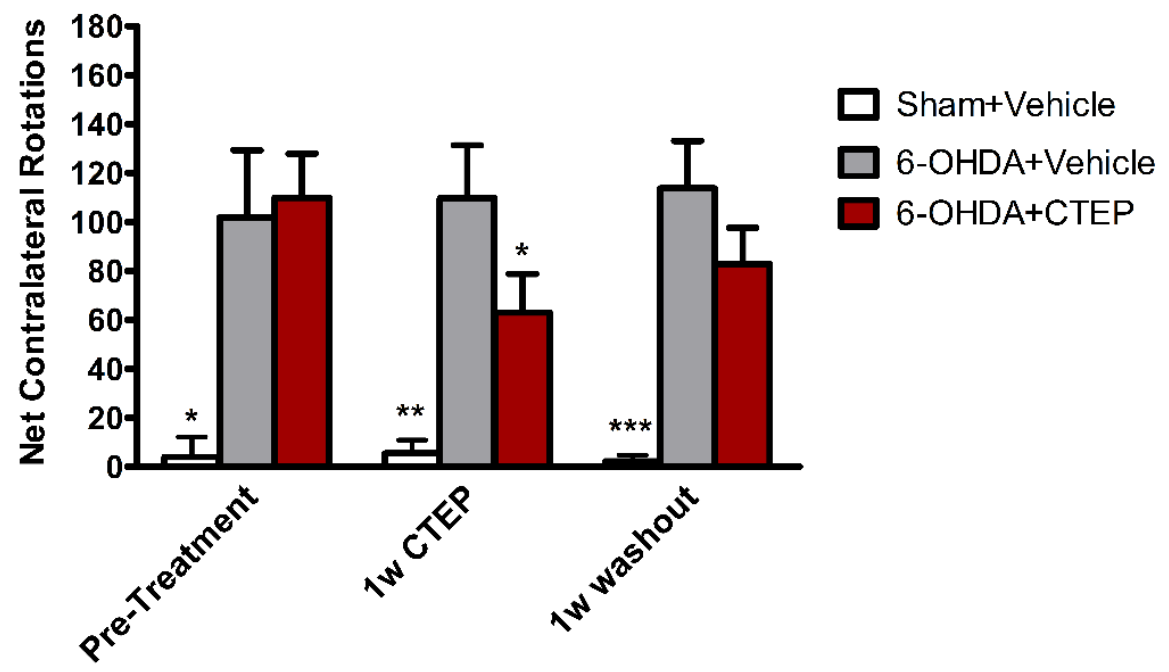

Figure 34 CTEP Washout Apomorphine Induced Rotations

Animals were given injections of apomorphine 30 days after 6-OHDA administration, 1 week after CTEP administration, and once again after a 1 week drug washout. The net contra-lateral rotations over a 20 minute period were recorded. A repeated measures ANOVA failed to reach significance, however a significant main effect was found $(F(2,21)=$ $22.05, \mathrm{p}<.01)$. Follow up comparisons of a priori hypotheses found that all 6-OHDA treated animals rotated significantly more than sham controls at the 30 day time point $(p<.05)$. After 1 week of CTEP administration 6-OHDA treated animal demonstrated significantly more contralateral rotations compared to sham controls. Similar to our findings in our 1 week model (Figure 8), 6-OHDA+CTEP treated animals demonstrated significantly less rotations compared to 6OHDA+Vehicle controls $(p<.05)$. However, after a 1 week drug washout there is modest regression, and the 6-OHDA+CTEP treated animals are no longer statistically different from 6-OHDA+Vehicle controls. 


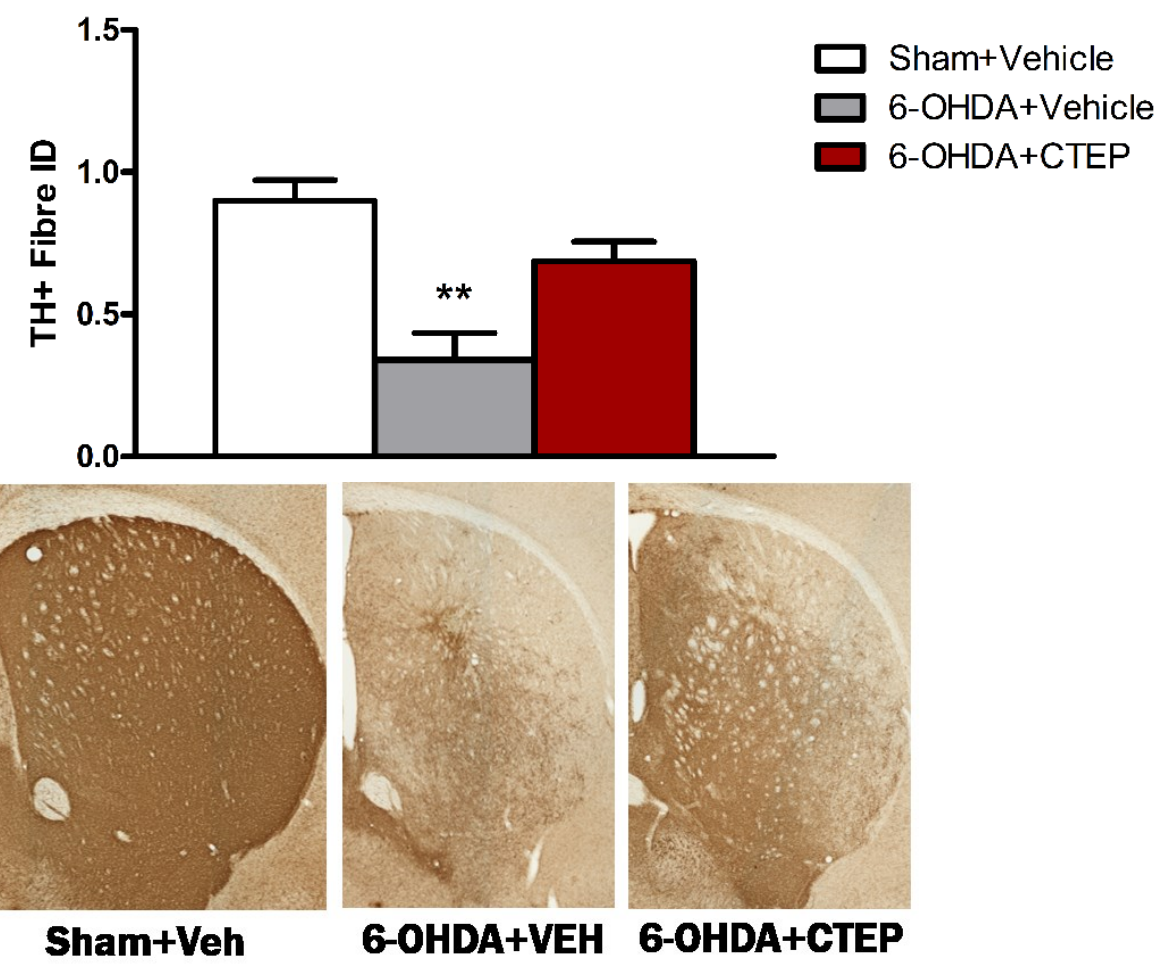

Figure 35 CTEP Washout Striatal TH+ Histology

The integrated density of striatal sections immunolabelled with $\mathrm{TH}$ was measured using ImageJ software after 1 week of CTEP administration followed by a 1 week drug washout period. A one way ANOVA found a significant main effect $(\mathrm{F}(2,11)=12.72, \mathrm{p}<.01)$. Post hoc analysis found that $6-\mathrm{OHDA}+$ Vehicle treated animals had significantly less $\mathrm{TH}+$ staining that all other groups $(\mathrm{p}<.01)$. 


\section{Rapamycin}

In the previous experiments, we identified the mTOR (more specifically mTORC1) signaling cascade as being a potentially important mediator of CTEP induced changes in the context of a 6OHDA induced lesion. To confirm the potential role of mTORC1 a partial recapitulation of the 1 week study was completed, however in addition to CTEP treatment some animals also received injections of rapamycin - a potent inhibitor of $\mathrm{mTORC} 1$. In this study animals received an i.p. injection of rapamycin every $48 \mathrm{hrs}$ in the evenings before CTEP treatment. The administration of these compound was timed so that rapamycin had sufficient time to be absorbed and cross the blood brain barrier to inhibit mTORC1 before CTEP could begin to activate it.

When comparing apomorphine induce rotation scores a one way ANOVA found a significant difference between the treatment groups $(\mathrm{F}(4,35)=8.59, \mathrm{p}<.0001)($ Figure 36$)$. The follow up comparisons

found that 6-OHDA increased the number of rotation $(p<.05)$ and CTEP once again reversed this effect. However, when rapamycin was 
also given it abolished the impact of CTEP, such that these animals were identical to controls.

With respect to $\mathrm{TH}+$ striatal staining density, a one way ANOVA found a significant main effect $(F(4,15)=9.28, p<.001)$ (Figure 37). The 6-OHDA treatment reduced striatal $\mathrm{TH}+$ labelling $(\mathrm{p}$ $<.05)$, while CTEP partially but significantly reversed this effect $(\mathrm{p}<$ $.05)$. Once again, the rapamycin treatment indicated that CTEP was likely acting through mTOR, as it significantly blocked the ability of CTEP to reverse $\mathrm{TH}+$ terminal loss $(\mathrm{p}<.05)$. Similarly, when comparing striatal BDNF levels, a significant difference was observed between the groups $(F(4,20)=16.93, p<.0001)($ Figure 38$)$. The follow ups, pinpointed a significance increase in animals that received the 6OHDA lesion in conjunction with CTEP, compared to all the remaining groups $(\mathrm{p}<.05)$. Most importantly, when mice also received rapamycin (in addition to CTEP and 6-OHDA) they did not differ from sham controls $(p<.001)$. Hence, the mTOR antagonist appeared to ablate the efficacy of CTEP. 
To confirm the efficiency of rapamycin treatment in blocking mTOR signaling, we assessed levels of its downstream effector, pp70S6K(Thr389). To this end, a significant main effect was found between the groups $(\mathrm{F}(4,20)=10.60, \mathrm{p}<.0001)($ Figure 39). The follow ups confirmed that rapamycin alone did indeed significantly lower $\mathrm{p}$ p70S6K $($ Thr389) relative to sham controls $(\mathrm{p}<.05)$. Moreover, as expected, CTEP increased levels of the p-p70S6K $($ Thr389) $(p<.05)$ and rapamycin prevented this effect. 


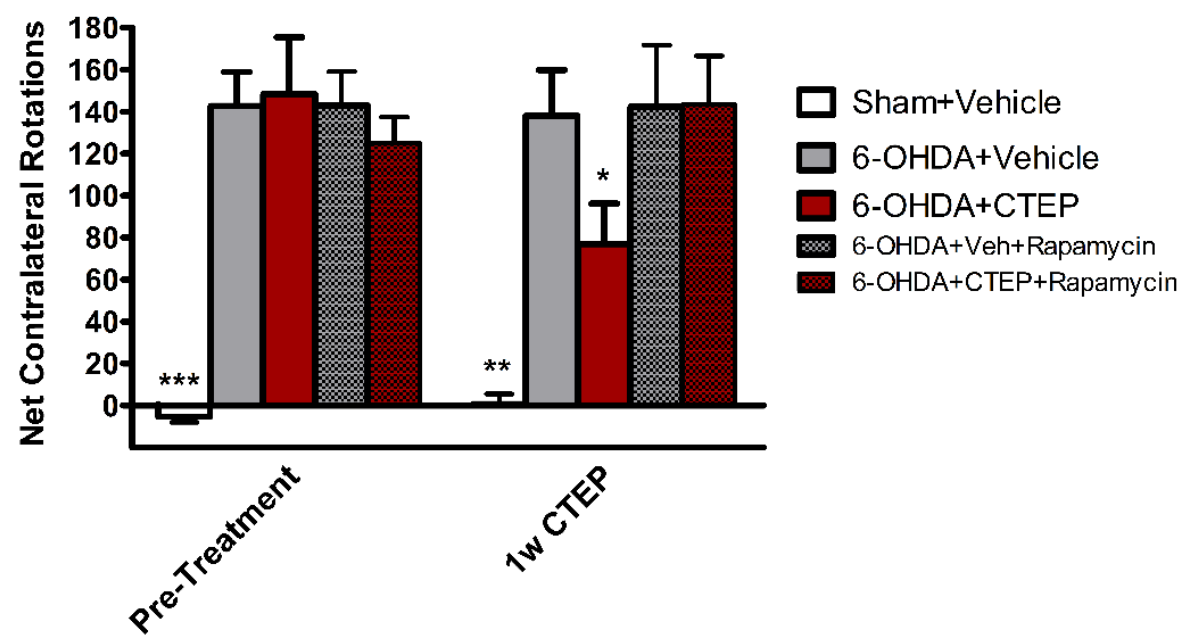

Figure 36 Rapamycin+CTEP Apomorphine Induced Rotations

Animals were given injections of apomorphine 30 days after 6-OHDA administration and again after 1 week of CTEP administration. At day 30 an ANOVA found a significant main effect $(\mathrm{F}(4,35)=15.146, \mathrm{p}<$ .0001). Post hoc analysis found that all 6-OHDA treated animals exhibited significantly more apomorphine induced contralateral rotations $(\mathrm{p}<.0001)$ compared to Sham+Vehicle controls. After 1 week of CTEP and/or Rapamycin administration an ANOVA found a significant effect $(\mathrm{F}(4,35)=8.59, \mathrm{p}<.0001)$. Follow up comparisons found that 6-OHDA+CTEP treated animals had significantly less apomorphine induced rotations compared to all other 6-OHDA treatment groups $(\mathrm{p}<.05)$. Importantly the addition of 6$\mathrm{OHDA}+\mathrm{CTEP}+$ Rapamycin prevents $6-\mathrm{OHDA}+\mathrm{CTEP}$ improvement $(\mathrm{p}$ $<.05)$. 


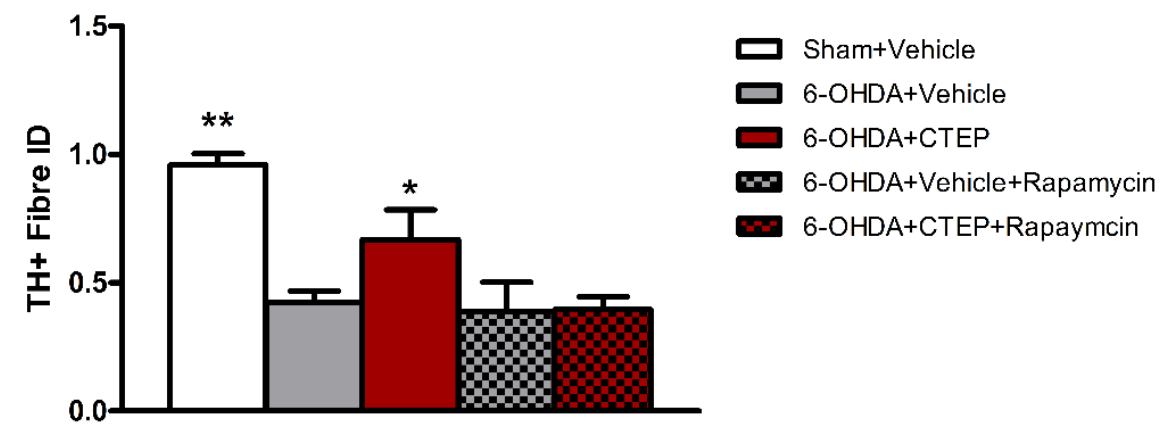

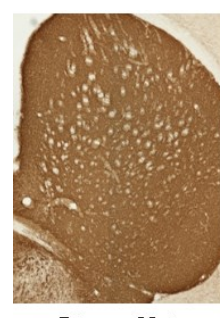

Sham+Veh

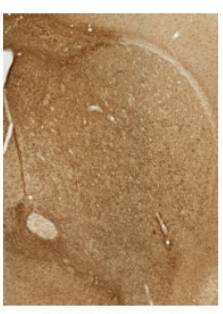

6OHDA+Veh

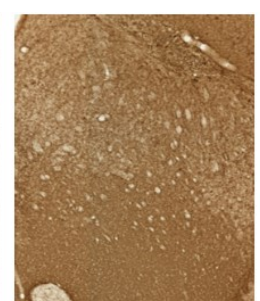

BOHDA+CTIP

Vehlcle

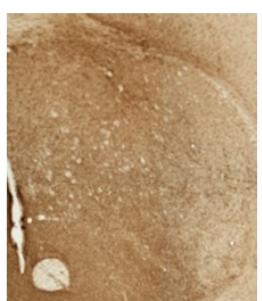

GOHDA+Veh

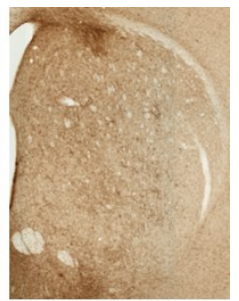

6OHDA+CTEP

Figure 37 Rapamycin+CTEP Striatal TH+ Histology

The integrated density of striatal sections immunolabelled with TH was measured using Image J software after 1 week of CTEP administration with or without combined Rapamycin injections. A one way ANOVA found a significant main effect $(\mathrm{F}(4,15)=9.28, \mathrm{p}<.001)$. Post hoc analysis found that all $6-$ OHDA treated animals had significantly less $\mathrm{TH}+$ staining compared to Sham+Vehicle controls. However, 6OHDA+CTEP treatment group had significantly more $\mathrm{TH}+$ staining compared to all other 6-OHDA treatment groups $(\mathrm{p}<.05)$. Importantly the addition of 6-OHDA+CTEP+Rapamycin prevents 6-OHDA+CTEP improvement $(\mathrm{p}<.05)$. 

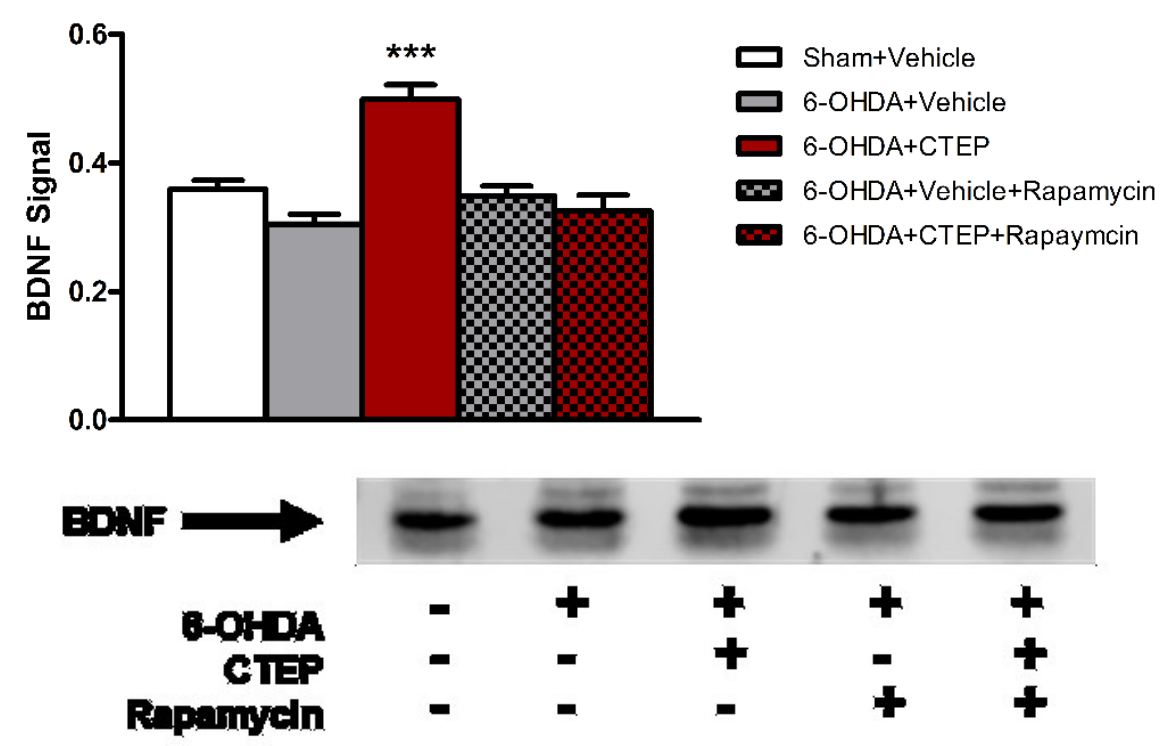

Figure 38 Rapamycin+CTEP Striatal BDNF

Striatal BDNF was measured after 1 week of CTEP administration with or without combined Rapamycin injections by western blot. A one way ANOVA found a significant main effect $(F(4,20)=16.93, p<.0001)$. Post hoc analysis found that 6-OHDA+CTEP treated animals had significantly more BDNF levels compared to all other treatment groups $(\mathrm{p}<.001)$. Importantly the addition of $6-\mathrm{OHDA}+\mathrm{CTEP}+\mathrm{Rapamycin}$ prevents the 6-OHDA+CTEP induced increase of BDNF $(\mathrm{p}<.001)$. This western blot was visualized using IR800 fluorophore on Licor Odyssey FC equipment, normalized total protein signal following a Fast Green Stain. 

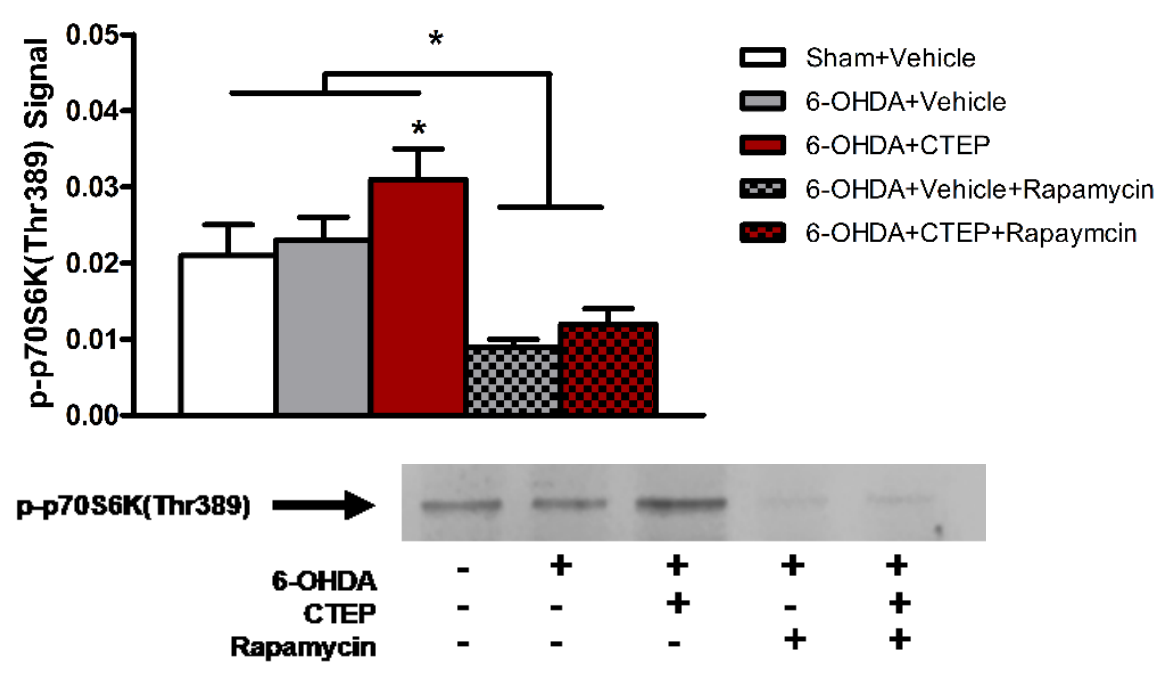

Figure 39 Rapamycin+CTEP Striatal pp70S6K(Thr389)

Striatal p-p70S6K(Thr389) was measured after 1 week of CTEP administration with or without combined Rapamycin injections by western blot. A one way ANOVA found a significant main effect $(\mathrm{F}(4,20)=10.60, \mathrm{p}<.0001)$. Post hoc analysis found that 6OHDA+CTEP treated animals had significantly more $p$ p70S6K(Thr389) levels compared to all other treatment groups ( $p<$ $.05)$. Rapamycin treated animals had significantly less pp70S6K(Thr389) levels compared to all other treatment groups ( $\mathrm{p}<$ .05). This western blot was visualized using IR800 fluorophore on Licor Odyssey FC equipment, normalized total protein signal following a Fast Green Stain. 


\section{Discussion}

\section{Rational}

Current therapies of PD do not reverse the degenerative course of the disease; rather they merely manage symptom severity. The primary method of treatment has not changed in more than 40 years and involves the use of L-DOPA, a dopamine precursor molecule that is capable of crossing the blood brain barrier (Yahr et al., 1969). Although initially very effective, the ability of L-DOPA to manage symptom severity decreases dramatically with prolonged use and disease progression, often increasing the severity of muscle dystonia and dyskinesias (Parkinson Study Group, 2000; Fahn et al., 2004).

Despite continual effort, there has been very few therapeutics that have been successfully translated from the laboratory to the clinic. Importantly, in many pre-clinical studies the efficacy of a potential therapeutic is tested in a manner that mostly tests its ability to prevent PD pathology - frequently researchers report that they start therapeutic administration prior to toxin administration. Unfortunately, this is not 
the case in the human condition - clinicians simply are not able to start therapeutics prior to disease initiation and or significant clinical progression. This is a major limitation in current literature. The argument can be made that in pre-clinical models, administering a potential therapeutic after PD-like pathology has occurred may result in more therapies being successfully translated into the clinic.

The majority of PD research is solely focused on the dopaminergic system. However, this might be short sighted as it is well known that other system are also affected and involved in the degenerative processes. In this regard, we are particularly interested in the glutamatergic system given its fundamental importance in numerous cellular processes that have been implicated in PD. Indeed, accumulating evidence shows that the glutamatergic neuronal system plays an important role in the etiology of PD (Greenamyre, 1986; Blandini et al., 1996). Specifically, glutamate has been reported to increase ROS production via auto-oxidation and in parallel, can provoke intracellular calcium release (Greenamyre, 1986; Blandini et al., 1996). Together, these processes are known to contribute to PD degeneration (Greenamyre, 1986; Blandini et al., 1996). Accordingly, targeting 
glutamatergic toxicity by modulating NMDA (Klockgether et al., 1996; Steece-Collier et al., 2000; Kelsey et al., 2004) and AMPA (Löschmann et al., 1991; Wachtel et al., 1992) receptor function has demonstrated beneficial effects in treating the symptomatic profile of PD. For instance, antagonism of NMDA receptors with the drug MK-801 has been reported to result in improvements in gait (Kelsey et al., 2004) and reduced apomorphine provoked rotations in 6-OHDA lesioned rats (Robinson et al., 2001). Similarly, administration of NMDA antagonists in MPTP treated monkeys reduced haloperidol associated catalepsy and other parkinsonian behaviours (ie. tremors, gait, posture, etc.) (SteeceCollier et al., 2000). As well, modulation of AMPA receptors with the antagonist NBQX also improved rotational and parkinsonian behaviours in 6-OHDA and MPTP models (Löschmann et al., 1991; Wachtel et al., 1992)

Much research in the past has focused on ionotropic NMDA and AMPA receptor modulation with great success in addressing PD symptomatology. However, translational into the clinic for treatment of neurodegeneration has been overall unsuccessful. More recently, a shift in focus more towards the modulation of metabotropic receptors has 
occurred. Most importantly, these receptors are potentially more robust targets as they impact a variety of crucial intracellular processes including stimulate G-protein dependent and independent pathways and also modulation of the aforementioned ionotropic receptors (Chen et al., 2007; Gerber et al., 2007; Ribeiro et al., 2017).

Of particular interest for the present thesis is the mGluR5 receptor system as a potential therapeutic target for PD. Indeed mGluR5 dysfunction has been implicated in various aspects of PD pathology including increased incidence of synucleinopathy (Price et al., 2010), PD related dyskinesia (Bezard et al., 2014), PD related striatal-cortical hyper excitability (Johnson et al., 2009), and general motor disturbances (Guimaraes et al., 2015). Furthermore, many the primary signalling partners of mGluR5 (e.g. ERK1/2, and PI3K/AKT/mTOR) have been previously described to have neuroprotective or other beneficial effects. For instance, ERK1/2 has been shown to positively affect protein translation as well as cell proliferation, migration, differentiation, and overall cell survival (Roskoski, 2012). mTOR has been described as being a mediator of cell survival and proliferation, due to not only its role in driving protein translation but also in mediating mitochondrial 
metabolism and cell structure re-organization (Fingar and Blenis, 2004; Jiang and Yeung, 2006; Jung et al., 2010; Morita et al., 2013). Additionally, mGluR5 is an important mediator of long term depression (LTD) and plasticity through its ability to directly modulate glutamatergic ionotropic receptors. Indeed, it does so by directly altering NMDA and AMPA surface expression, as well as driving the release of intracellular calcium stores (Volk et al., 2006; Purgert et al., 2014). Hence, there are many mechanisms through which mGluR5 modulation could impart recovery in PD.

Selective pharmacological modulation of $\mathrm{G}$ protein coupled receptors is extremely challenging, due mostly to the homogeneity of the orthostatic binding site found in the various subtypes within receptor families. Thus, by targeting such a ubiquitous bindings site it would be expected to have numerous potentially deleterious off target effects. However, advances in protein tomography have allowed for the identification of subtype specific allosteric binding sites that are distinct from the traditional orthostatic binding locations. It is in these sites that designer allosteric modulators can bind and alter receptor function. One such allosteric regulator compound is CTEP (2-chloro-4-((2,5- 
dimethyl-1-(4-(trifluoromethoxy)phenyl)-1H-imidazol-4-

yl)ethynyl)pyridine). This is a potent negative allosteric modulator with very high selectivity to mGluR5 (Lindemann et al., 2011). CTEP binds to mGluR5 with $>1000$ fold selectivity compared to over 100 other targets, including all known mGluRs (Lindemann et al., 2011). CTEP is oral bioavailable and can efficiently cross the blood brain barrier (Lindemann et al., 2011; Michalon et al., 2012).

This thesis explored the therapeutic potential of CTEP in a 6OHDA model of PD. We are particularly interested in neuro-recovery following a nigrostriatal lesion and how CTEP might modulate neurotrophic processes within the striatal-cortical system that is dysregulated in PD.

We initially sought to confirm the reliability our 6-OHDA model. To this end, our data clearly indicated that $20 \mu \mathrm{g}$ of $6-\mathrm{OHDA}$ induced a robust and replicable lesion in CD1 mice, resulting in $\sim 60 \%$ of striatal area and greater than $50 \%$ loss of SNc neurons. After 30 days, various behavioural deficits were observed, including decreased motor coordination when tested on the accelerating rota rod, and dramatic 
increase in net contralateral rotations when challenged with apomorphine. This model is well tolerated by the animals, with minimal long term post-surgical complications. Most importantly, when given following the establishment of the 6-OHDA lesion, CTEP reversed the behavioural deficits and appeared to promote a re-innervation of $\mathrm{TH}+$ terminals within the striatum. In the following sections of this discussion, we will discuss these findings, along with the evidence that these effects were mediated by the mTOR pathway.

\section{Overview of Findings}

The first CTEP experiments investigated the short term (1 week) and long term (12 weeks) effects of the drug. As CTEP is oral bioavailable (Lindemann et al., 2011) we developed an administration paradigm using chocolate pudding, in which the mice readily consumed. The dose we administered via the pudding suspension, $2 \mathrm{mg} / \mathrm{kg}$ every $48 \mathrm{hrs}$, was previously found to result in approximately $75-80 \%$ receptor occupancy in the striatum and cortex of adult mice (Lindemann et al., 2011). Furthermore, at this dosing CTEP can be detected in brain lysates 
over 50hrs after administration (Lindemann et al., 2011). As such, this dosing schedule provides continual receptor occupancy.

The fact that CTEP improved behavioural deficits after only 1 week and was even more effective after further dosing, indicates that the drug might have fairly rapid clinical efficacy. As 12 weeks of CTEP treatment completely abolished apomorphine induce rotational behaviour, it appears that repeated dosing can boost overall drug efficacy and could reflect progressive or adaptive brain changes. Also, of importance is the fact that CTEP not only reversed motor deficits, but also cognitive deficiencies in a reversal Morris water maze. This is in keeping with previous studies that found CTEP similarly improved spatial memory and object recognition in an Alzheimer's animal model (Hamilton et al., 2016). CTEP was also found to impart both motor and cognitive improvements in models of Huntington's disease (Abdelrahman et al., 2017). Hence, CTEP clearly has broad ranging effects that could be therapeutically important for multiple symptom clusters. It is also important to underscore that in the current studies we found that CTEP alone did not have behavioural consequences; rather its effects were only evident in the presence of a 6-OHDA lesion. This 
suggest that CTEP is preferentially acting in the context of an insult that would clearly be stressing the surviving cells.

It is significant that CTEP rescued striatal $\mathrm{TH}+$ terminal loss following 6-OHDA treatment but had no effect on the loss of SNc neurons. This suggests that, as hypothesized, CTEP appears to have provoked neuroplasticity changes in surviving axons/dendrites but did not induce any neurogenic effects at the level of the dopaminergic soma. Accompanying these striatal changes, CTEP also reversed the 6-OHDA induced elevations of striatal and motor cortex FosB levels. This suggests that the re-innervation induced by CTEP was functionally important and hence normalized postsynaptic activity. Indeed, FosB plays many roles in the cell, however it is commonly used as a marker of overall cell activity - in part due to its role as an activity dependent transcription factor.

Normally the loss of dopaminergic input to the striatum results in a hyperactivity of the GABAergic striatal interneurons, as was reflected in the current 6-OHDA induced FosB elevation. The hyperactivity is accompanied by imbalanced D1 and D2 receptor 
activation on the interneurons. For example, in the early stages of disease there is a marked reduction of D2 receptors, resulting in limited D2 mediated inhibition (Hisahara and Shimohama, 2011). As the balance of receptors shift to predominantly D1, striatal medium spiny neurons lose the inhibitory control imparted by $\mathrm{D} 2$, ultimately resulting in increased striatal activity (Galvan and Wichmann, 2008; Hisahara and Shimohama, 2011). The loss of the inhibitory D2 receptor control results in changes throughout the basal ganglia regions and the motor cortex. Indeed, this has been shown to be true in both animal models and PD patients (Mehler-Wex et al., 2006).

In order to mechanistically identify how CTEP is acting within the context of the 6-OHDA lesion, we assessed several mGluR5 related signaling cascades. In particular, mGluR5 can signal through three prominent pathways that are relevant for neuroplasticity and neuronal recovery; these are CaMKII, ERK 1/2 and mTOR. Within the striatum, p-CaMKII $\alpha(\operatorname{Thr} 286)$ and p-ERK1/2(Tyr204) were mostly unaffected with the exception of $\mathrm{p}-\mathrm{CaMKII} \alpha(\mathrm{Thr} 286)$ being reduced by 6-OHDA and this effect was reversed by CTEP at only the 1 week time. The exact role of p-CaMKII $\alpha(\mathrm{Thr} 286)$ in the striatum in this context is unknown. 
The most marked effects of CTEP were with regard to the mTOR pathway. Indeed, CTEP greatly increased levels of p-mTOR(Ser2448) after only 1 week of drug administration. Although blunted, levels of pmTOR(Ser2448) remained elevated in the 12 week chronic model. The serine 2448 site on the mTOR complex is the primary binding site of $\mathrm{AKT}$, and is often indicative of mTORC1 activation (Chiang and Abraham, 2005). While not measured directly, it should be noted serine 2448 is an important activation site of the mGluR5/PI3K/AKT signalling cascade. Despite mTOR phosphorylation at serine 2488 being associated with mTORC1 activity, there is also evidence indicating that p-mTOR(Ser2448) is capable of binding to both raptor and rictor, allowing it to form both mTORC1 and mTORC2 complexes respectively (Rosner et al., 2010). Normally, raptor forms part of mTORC1, whereas rictor forms mTORC2, which differentially influence downstream signaling. To determine which of these was being activated, we assessed the mTORC1 downstream effector p70S6K, which is specific for this complex. To this end, after both the 1 week and 12 week CTEP administration treatment schedules there was a marked increase in p-p70S6K(Thr389). 
Phosphorylation of p70S6K can increase protein translation that is critical for neuronal plasticity and remodelling (Switon et al., 2016), as would be required for recovery following exposure to toxicants, such as 6-OHDA. p70S6K accomplishes this by phosphorylating eEF2k, a protein which normally acts as a ribosomal suppressor of protein translation (Switon et al., 2016). Additionally, p70S6K activates eIF4B and represses PDCD4 through phosphorylation, resulting in activation and disinhibition of another important mediator of protein translation, eIF4A (Switon et al., 2016). In addition to its role as a mediator of protein translation, p70S6k is also known to stimulate phosphorylation of mTOR at serine 2448 , thereby creating a positive feedback loop (Switon et al., 2016).

Because mTOR is such as critical component of a multitude of cellular functioning ranging from mitochondrial homeostasis to microtubule organization, autophagy, and even apoptosis, it is difficult to determine exactly which of the proteins it regulates would be most important in our model. However, one particularly notable protein product of mTOR driven protein translation is brain derived 
neurotrophic factor (BDNF) (Maiese, 2015). Indeed, we found that BDNF was increased following either of the 1 or 12 week CTEP treatments, but only in the context of the 6-OHDA lesion. It is particularly notable that BDNF levels were found to vary in parallel with the aforementioned mTOR and p70S6K. This lead us to hypothesize that this growth factor might have been fundamental for the striatal reinnervation we presently observed.

BDNF is a member of the neurotrophin family of proteins and signals through the tyrosine kinase B (TrkB) receptor system. Furthermore, BDNF is the most widely expressed neurotrophin in the mammalian brain (Kolbeck et al., 1999). When BDNF binds to TrkB the receptor dimerizes and initiates a signalling cascade (Sasi et al., 2017). Interestingly, stimulation of the TrkB receptor results in changes in many of the same signaling partners affected by mGluR5; specifically, PI3K/AKT/mTOR, ERK1/2, and CaMKII (Sasi et al., 2017). In addition to these pathways, activation of the TrkB receptor also stimulates the activation of CREB, an important cellular transcription factor (Sasi et al., 2017). Through these cascades, $\mathrm{BDNF} /$ TrkB has been shown to modulate protein translation, gene 
transcription, excitability, calcium signalling, and cellular remodelling (Binder and Scharfman, 2004; Sasi et al., 2017). Additionally, BDNF has been shown to directly modulate glutamatergic receptors, potentiating neuroplasticity by modulating NMDA and AMPA receptor activity and expression (Carvalho et al., 2009).

BDNF may play a role in regulating normal dopamine neuronal activity (Murer et al., 2001). In fact, nigral administration of BDNF in healthy rats results in rotational behaviours (Altar et al., 1992; MartinIverson et al., 1994; Martin-Iverson and Altar, 1996), increased nigral neuron firing rate (Shen et al., 1994) and increased dopamine turnover (Lapchak et al., 1993; Hagg, 1998; Lau et al., 1998). With respect to PD, BDNF has been reported to be depleted in the striatum and the substantia nigra (Murer et al., 2001). Furthermore, the prominent A30P and A53T mutations of $\alpha$-synuclein that are implicated in PD can decrease levels of BDNF (Kohno et al., 2004). Conversely, many PD animal models have demonstrated that BDNF administration can protect nigrostriatal dopamine neurons against toxin induced degeneration (Frim et al., 1994; Levivier et al., 1995; Shults et al., 1995; Tsukahara et al., 1995; Hung and Lee, 1996) . 
Unfortunately, systemic administration of BDNF is not clinically viable because of its limited BBB permeability and potentially severe side effects. While studies investigating the side effects of BDNF are limited, many researchers and clinicians are cautious given that administration of trophic factors in general can induce cellular hyperplasia and nerve sprouting (Winkler et al., 1997), hypophagia and concomitant weight loss (Williams, 1991; Eriksdotter Jönhagen et al., 1998), and hyperinnervation of cerebral blood vessels (Isaacson et al., 1990). Given these limitations, it is of interest to utilize other pharmacological means to stimulate the expression of endogenous neurotrophic factors such as BDNF (Barber et al., 2015).

When taken together, our findings are consistent with the proposition that CTEP provoked modulation of mGluR5 can stimulate pro-recovery process in the context of a PD relevant lesion. Specifically, the recovery of the striatal dopaminergic terminals was correlated with behavioural recovery. Given our data indicating that mTORC1 appears to be involved, we next specifically blocked this pathway in order to confirm its role in the recovery effects of CTEP. To this end, rapamycin, 
a well-known inhibitor of mTORC1, was administered. Rapamycin exerts effects by first binding to the FRB domain of mTOR. This binding then can result in an allosteric change in mTORs catalytic binding site (Jacinto et al., 2004). Importantly, the FRB domain on mTORC1 is affected, whereas this domain is not accessible in the mTORC2 form, and therefore is not directly affected by rapamycin, (Sarbassov et al., 2006).

As rapamycin has a biological half life of approximately $60 \mathrm{hrs,}$ we chose a dosing schedule, wherein rapamycin was given every $48 \mathrm{hrs}$ prior to each CTEP injection. Essentially, we hoped to effectively block any potential mGluR5 mediated increases in mTORC1. Indeed, we did find that rapamycin alone (with 4 injections over 1 week) reduced striatal p-p70S6K(Thr389) levels indicating that mTORC1 was blocked. Most importantly however, rapamycin also blocked the ability of CTEP to reduce apomorphine induced rotations. Similarly, rapamycin pretreatment blocked the re-innervation of striatal terminals and increase in BDNF previously seen to be induced by CTEP treatment. 
The rapamycin study supports the idea that CTEP imparts its beneficial effects through striatal mTOR activation. While we cannot discount that CTEP may be acting through other potential molecular mediators, these data strongly suggest that mTORC1 activation is required in some capacity for dopaminergic striatal recovery. Indeed, activation of $\mathrm{mTOR} / \mathrm{p} 70 \mathrm{~S} 6 \mathrm{~K}$ has been shown to induce fibre regrowth following traumatic injury (Abe et al., 2010; Yang et al., 2014; Hu, 2015). Similarly removal of PTEN, an important inhibitor of mTOR, has been shown to enhance neurite outgrowth of nigrostriatal DA neurons in a mouse model of PD (Zhang et al., 2012). Furthermore, it is important to underscore that while we found increases in BDNF, this most likely not the only post-transcriptional mTORC1 target that might have been modified by CTEP. Indeed, mTOR has been shown to increase other trophic factors, including epidermal growth factor (EGF), fibroblast growth factor (FGF), and erythropoietin (EPO) (Maiese, 2015). Additionally, our own data demonstrates that CTEP results in modulation of different signalling cascades in different brain regions. For example, it will be recalled that in the motor cortex CTEP affected p-ERK1/2(Tyr204) and p-CaMKII $\alpha(T h r 286)$ after both 1 and 12 weeks 
of drug administration. This was in contrast to the striatum, wherein mTOR was the primary effector.

The elevation of $\mathrm{p}$-ERK1/2(Tyr204) that was prompted by the 6-OHDA and CTEP treatments might contribute to the functional recovery effects observed (Roskoski, 2012). Indeed, it has been shown that mGluR5 mediated increases of ERK1/2 stimulate transcription factors that have important neuroplastic effects, such as ELK-1 and CREB (Gladding et al., 2009). ELK-1 and CREB have been shown to increase neuroplasticity through the protein Arc/Arg3.1 (Gladding et al., 2009; Kumar et al., 2012). Importantly, increases in Arc have been shown to regulate spine density and morphology (Peebles et al., 2010) and several other aspects of synaptic plasticity, including long term depression (Chowdhury et al., 2006; Rial Verde et al., 2006). Similarly, ERK1/2 has been shown to stimulate the internalization of NMDA and AMPA receptors, which again could conceivably underlie critical changes in neuroplasticity (Chowdhury et al., 2006; Rial Verde et al., 2006; Gladding et al., 2009). 
Importantly, this data demonstrates that CTEP is able to act differently in distinct brain regions and that it only does so in the context of the lesion. Additionally, our data suggest that mGluR modulation only begins to manifest functional changes when it interacts with cells already in distress. This is important as it may reduce potential off target side effects and may only modulate regions specifically under stress.

Finally, the last study investigated whether CTEP imparts relatively long term changes. In this study, we found that after a 1 week drug washout, there was a modest lost of CTEP efficacy with regard to apomorphine induced rotations, such that the drug no longer significantly affected this behavioural outcome. Despite this loss of behavioural efficacy, the impact of CTEP on dopaminergic $\mathrm{TH}+$ fibre density in the striatum was still apparent. This indicates that modulation of mGluR5 with CTEP was able to stimulate striatal re-innervation, but continual modulation of the receptor is required to maintain the improved functional outcomes. 


\section{Comparable Pre-Clinical Therapeutics}

To date, several mGluR5 NAMs have been developed, including MPEP, MTEP, and fenobam. While all these compounds target mGluR5, they all have variable potency, penetration rates, and metabolism. The vast majority of the current pre-clinical research surrounds the use of mGluR5 modulation as a therapeutic option to treat levodopa induced dyskinesias (LIDs). Indeed, it has been shown that targeted modulation of mGluR5 receptors in downstream regions of the substantia nigra (i.e. striatum, thalamus, cortex) can compensate for dopamine receptor hypersensitivity seen with prolonged levodopa treatments (Maranis et al., 2012). MPEP, MTEP, and Fenobam have been shown to be effective in treating LIDs in both rats (Ambrosi et al., 2010; Marin et al., 2011; Bashkatova and Sudakov, 2012; Hsieh et al., 2012; Maranis et al., 2012; Iderberg et al., 2013; Ferrigno et al., 2015) and non-human primates (Johnston et al., 2010; Morin et al., 2010, 2013a; Rylander et al., 2010; Masilamoni et al., 2011; Ko et al., 2014).

With respect to treating dyskinesias, it appears that modulating mGluR5 with these select NAMs prevents levodopa induced increases 
in various receptors, including D1 receptor (Morin et al., 2014), NMDA, AMPA, and even mGluR2/3/5 (Morin et al., 2013b). Interestingly, modulation of other mGluRs has also shown to be beneficial in treating various akinetic and dyskinetic behaviours. In contrast to negative modulation of mGluR5, stimulation or modulation with PAMs seems most effective in Group II and III mGluRs. This is due to the differences in receptor location and role. For example, Group II receptors are highly expressed in the upstream substantia nigra reticulata (Nicoletti et al., 2011). Group III receptors are highly expressed in the striatum however act as pre-synaptic inhibitors of gabaergic medium spiny neurons (Nicoletti et al., 2011). In both cases, an increase in receptor activity counters aberrant basal ganglia activity seen in PD. Indeed, stimulation of Group II mGluRs with the agonist 2R,4R-APDC decreased the number of apomorphine induced contralateral rotations in 6-OHDA treated rats (Chan et al., 2010). Broad spectrum Group III mGluR agonists L-AP4 and ACPT-I improved the overall behavioural phenotype seen in 6-OHDA treated animals, however also resulted in drug related dystonic posturing (Lopez et al., 2012; Sun et al., 2013). Targeted modulation of Group III mGluR4 with various PAMs has been shown to improve akinesia seen in both 6-OHDA and mitopark PD 
models (Jones et al., 2011; Le Poul et al., 2012; Bennouar et al., 2013). However in some cases, mGluR4 PAMs work most effectively as preventative therapeutics, having limited capacity to reverse already developed phenotypes (Lopez et al., 2011; Betts et al., 2012; Iderberg et al., 2015).

Although most studies have been restricted to assessing the ability of mGluRs in reducing L-DOPA induced side effects (i.e. dyskinesias), a few reports have assessed mGluRs and primary PD neurodegeneration. For instance, striatal recovery and neuroprotection from 6-OHDA toxicity has been shown following delayed administration of the Group II mGluR agonist 2R,4R-APDC (Chan et al., 2010). Similarly, Betts et. al. (2012) found that administration of the Group III mGluR4 PAM VU0155041 before 6-OHDA administration results in neuroprotection of both the $\mathrm{SNc}$ and striatum. Another study found that administration of the broad spectrum Group III mGluR agonist L-AP4 before 6-OHDA administration reduces toxin induced degeneration (Austin et al., 2010). Importantly, although beneficial all of these studies involved direct infusion of the mGluR acting drugs into the SNc. This is obviously a major clinical caveat. 
Focusing on mGluR5, nearly all the current literature uses a preventative model design; i.e. the drug administration begins either before or in parallel to the toxin administration. Masilamoni (2011) found that starting MTEP therapy 5 days before administering MPTP in monkeys results in protection of nigrostriatal dopaminergic neurons and striatal projections. Another study showed that administration of 6OHDA followed immediately by 28 day continual minipump infusion of MPEP into the striatum results in protection of nigral $\mathrm{TH}+$ neurons, and prevents rotational behaviour following sub-threshold doses of levodopa (Fuzzati-Armentero et al., 2015). Similarly, daily intraperitoneal injections of MPEP for 14 consecutive days immediately following nigral administration of 6-OHDA prevented the loss of TH+ nigral neurons in both the SNc and VTA (Chen et al., 2011). This study also found that MPEP rescues 6-OHDA deficiencies in the elevated plus maze and social interaction tests (Chen et al., 2011). Yet another study has shown that daily intraperitoneal injections of MPEP for 14 consecutive days starting 24 hours after nigral administration of MPTP prevents nigral $\mathrm{TH}+$ cell loss, and significantly reduces striatal terminal loss compared to saline controls (Hsieh et al., 2012). In addition to 
protection of the dopaminergic nigral system, it was found that MPEP prevents MPTP induce microglial reactivity. However, Ferrigno (2015) found that delaying therapeutic administration by 4 weeks negates any protective effects previously seen with MPEP.

It is very important at this juncture to underscore the fact that there are no pre-clinical data whatsoever regarding the use of CTEP in a PD model other than what is presented in this thesis. Secondly, CTEP has increased brain penetrance and persists longer in the brain than all of the aforementioned studies using other mGluR5 acting drugs. Thirdly and most importantly, only one other lab has investigated mGluR5 modulation with regards to recovery processes using a delayed onset model (Ferrigno et al., 2015). However, this study used MPEP with repeated i.p. injections and was not successful in demonstrating any behavioural improvements (Ferrigno et al., 2015). In contrast, we utilized CTEP and used a novel oral administration paradigm. Using this procedure, we are able to closely monitor dosing and achieve stable levels of the drug and avoid the unnecessary stress of repeated injections. We hope that this ensures not only more robust and stable 
brain changes but also should facilitate more accurate behavioural analyses.

\section{Potential Clinical translation}

CTEP is an effective NAM of rodent and human mGluR5 and was developed by the parent company Roche (Lindemann et al., 2011). Compared to the other mGluR5 modulators; namely fenobam, MPEP, and MTEP, CTEP binds more readily to mGluR5, has a higher brain penetrance, and persists in the brain more than ten times longer (Lindemann et al., 2011). Despite CTEP's ability to modulate human mGluR5, the compound itself has not been used in clinical trials. Instead, the structurally similar compound Basimglurant has been used by Roche in its clinical investigations. CTEP and Basimglurant differ by only one functional group; CTEP containing a trifluoromethyl group whereas Basimglurant has only a single fluoride group. CTEP and Basimglurant are both NAMs of mGluR5 and both have inverse agonist potential (Lindemann et al., 2011, 2015). Comparatively, Basimglurant does result in increased receptor occupancy in the striatum and motor cortex ( $>90 \%$ ), and remains in the human body longer than CTEP 
(Lindemann et al., 2015). Conversely, CTEP remains in the rodent body longer than Basimglurant. Because of this, CTEP is used in pre-clinical animal experiments and Basimglurant is used in human clinical trials. Novartis has also developed an mGluR5 NAM, mavoglurant, which is frequently compared to CTEP. Functionally mavoglurant behaves similar to CTEP and Basimglurant, with the exception of metabolic clearance rate which is more similar to MPEP/MTEP/Fenobam, persisting only in the brain for approximately 8 hours (Vranesic et al., 2014).

Many of these mGluR5 NAMs were first tested as potential treatments of Fragile X Syndrome. Fragile X syndrome is an inheritable genetic disorder that results in intellectual disability (Pop et al., 2014). Evidence has shown that mutations in the affected FMRP gene leads to increased mGluR5 activity, resulting in aberrant protein synthesis and synaptic plasticity processes (Pop et al., 2014). Pre-clinical evidence demonstrated that modulating mGluR5 with CTEP was effective in correcting the Fragile X phenotype in mouse models (Michalon et al., 2012). However, multiple clinical trials testing the efficacy of Basimglurant in Fragile $\mathrm{X}$ failed to meet primary outcomes (Clinical 
Trials: NCT01015430, NCT01517698, NCT01750957). Similarly, mavoglurant, the mGluR5 NAM developed by Novartis, also failed to meet primary outcomes in patients with Fragile X (Clinical Trials: NCT00718341, NCT01253629, NCT01348087, NCT01357239, NCT01433354). Basimglurant has also recently been tested for the treatment of major depression, however while initially promising this too has ultimately demonstrated limited efficacy in humans (Quiroz et al., 2016). Similarly, mavoglurant has been tested in clinical trials for obsessive compulsive disorders, however again this trial was terminated early for failing to meet early outcome measures (Clinical Trial: NCT01813019).

Clinical trials using mGluR5 modulation for motor disorders have been limited. In this regard, Mavoglurant failed to improve the chorea symptoms in patients with Huntington's disease (Reilmann et al., 2015). With respect to levodopa-induced dyskinesias (LID), mavoglurant showed initial promise (Berg et al., 2011), however an expanded phase II clinical trial demonstrated that mavoglurant actually did not improve LIDs (Stocchi et al., 2013; Trenkwalder et al., 2016). With these findings in hand, Novartis stopped all testing of mavoglurant 
in their movement disorder therapeutic pipeline. Shortly afterwards Roche followed suit, never actually completing a clinical trial using Basimglurant as a potential therapeutic for a movement disorder. In contrast, Addex Therapeutics Dipraglurant has shown modest improvements in reversing LIDs in a recent phase 2a clinical trial (Tison et al., 2016). Unlike Novartis and Roche, Addex is pressing forward in an expanded phase $2 \mathrm{~b}$ clinical trial.

Exactly why these pharmacological compounds have failed to successfully translate to the clinic is not clear. This is especially surprising as many of the pre-clinical results have been validated by multiple different research groups. In the case of Fragile X syndrome, Lindemann's group have proposed multiple potential explanations (Scharf et al., 2015). Firstly, that obvious limitation of animal models could be a potential factor. Specifically, despite Fragile X being a monogenic disorder, it is likely that altering the gene in rodents does not result in identical changes observed in humans. Secondly, are the limitations imposed by the clinical study design. Upon retrospective analysis, Lindemann argues that the clinical trials could have been better structured in a manner that more effectively tests their experimental 
questions. And finally, is the concern about appropriate timing of when to administer the drugs. Lindemann's group argues as Fragile $\mathrm{X}$ is a developmental disease early intervention is required to maximized pharmacological potential.

The three factors which Lindemann's group states may have caused the discordance between pre-clinical and clinical results may also ring true in the case of PD. Indeed, there exists no one true model of PD. Each model is only capable of replicating certain clinical features. Because of this, each model is limited with respect to potential clinical translation. Second and third points proposed by Lindemann regarding clinical trial design and timing of the drug administration in this case can be addressed together. In particular, the patients used in the clinical trials may have been at too late a stage for the drug treatment to be maximally efficacious. Indeed, patients were diagnosed about 12 years earlier, had been on dopamine replacement therapy for an average of 10 years, and were showing dyskinesias for the preceding 4 years prior to the start of the experiment (Stocchi et al., 2013; Trenkwalder et al., 2016). Together, this suggests that the average patient enrolled in the clinical trials patients may be considered mid-stage in disease 
progression. Perhaps more pronounced effects would have been evident in a patient population with a less progressed disease state.

Appropriate timing is of utmost importance, such that any clinical intervention should be started as early as possible. In the case of $\mathrm{PD}$, much of the dopaminergic system is already lost by the time that patients reach the clinic (Bernheimer et al., 1973; Toulouse and Sullivan, 2008). At such stages, the remaining neurons are likely incredible vulnerable and could easily developed hypersensitivity to levodopa. More relevant to the present thesis, the capacity for the reinnervation of the striatum likely decreases at advanced stages of the disease. This would of course, diminish the likelihood of successful treatment translation. This was one of the reasons we choses to titrate our lesion to a moderate level $\sim 60 \%$ loss of striatal terminals, which causes behavioural impairment, but animals remain reasonably healthy. In our model, motor impairments were not basally apparent and only become evident when animals are behaviourally challenged. As such, we suggest that perhaps more successful clinical translation in PD using mGluR5 NAMs can be observed with earlier intervention. 


\section{Future Directions}

Despite the high affinity of CTEP for mGluR5, it conceivably could bind to other GPCRs. While Lindemann (2011) did test CTEPs affinity to many different receptors, their screening panel was not exhaustive. For this reason, it would be useful to repeat some of the present work in mGluR5 knock out (KO) mice to ensure that this receptor is indeed critical. However, this study is not without its logistical pitfalls. It has been shown that a mGluR5 $\mathrm{KO}$ alone is sufficient to induce a neuroprotective phenotype in the 6-OHDA mouse model of PD (Black et al., 2010). Knocking out mGluR5 does not confer complete protection against 6-OHDA toxicity, and still results in behavioural and neurochemical changes compared to sham controls. To the best of my knowledge, a direct comparison of WT and mGluR5 KO has not been completed in any other models of PD. However, as most models rely on neuronal death due to oxidative stress/damage it may be presumed that a mGluR5 $\mathrm{KO}$ would have similar effects as seen in the 6-OHDA treated animals. 
This thesis used the 6-OHDA toxin model of PD due to its effectiveness at inducing nigrostriatal degeneration, its replicability between animals and experiments, and its relative ease of application. 6-OHDA is an excellent model for early proof of concept pre-clinical experimentation. However, 6-OHDA is not a perfect model of PD and lacks key pathological hallmarks. As such future work should work beyond the 6-OHDA model. Genetic models may offer additional information beyond the toxin-based approaches. One potential model would be the PINK1 KO rat model of PD. Current published data demonstrate that over an 8 month period there is approximately $50 \%$ loss in TH-positive neurons, increase in $\alpha$-synuclein aggregation, and changes in various vocal and oromotor functions that precede any agedependent gait and motor impairments (Dave et al., 2014; Grant et al., 2015; Kelm-Nelson et al., 2015; Villeneuve et al., 2016). Unpublished data from collaborators at the University of Ottawa demonstrate that these pathological and phenotypic changes continue to progress with age, demonstrating significant striatal dopamine loss and more pronounced behavioural phenotype beyond 12 months of age (Park, unpublished). The use of the PINK1 KO genetic model would be ideal 
to further confirm CTEPs efficacy in PD, as this model is more akin to the human disease.

Moving beyond validation of CTEP in other models of PD, other important questions remain. First and foremost, would be investigating if CTEP modulates mTORC2. While only mTORC1 related mTOR(ser2448) was probed in this thesis, there is a strong likelihood that mTOR is being phosphorylated at other sites. Indeed, mTORC2 activation could have important disease modifying roles by driving structural re-organization through Rho/Rac signaling cascades (Li et al., 2004) and further activation of mTORC1 via AKT (Huang and Manning, 2009). While the results of the Rapamycin study suggest that mTORC1 is necessary and required for CTEP mediated improvement assuming that mTORC1 is working in isolation would be naïve. Similarly, the roles of ERK1/2 and CamKII $\alpha$ in the 6-OHDA+CTEP treated animals needs to be further investigated. It is very likely that both ERK1/2 and CamKII $\alpha$ have supportive roles in addition to the observed mTOR activation. To address this, experiments using antagonists for ERK1/2 and CamKII $\alpha$ should be used. For example, FR180204 and 
KN-93 could be used to block ERK1/2 (Gokce et al., 2009) and CamKII (Vest et al., 2010; Pellicena and Schulman, 2014) activity respectively.

This thesis demonstrated that CTEP was able to stimulate striatal dopaminergic terminal regrowth. Additionally, the alterations of overall neuron activity levels in downstream regions such as motor cortex suggest large changes to the basal ganglia circuitry. These conclusions are derived from changes in overall $\mathrm{FosB}+$ reactivity and marked changes in behavioural phenotype. While FosB is an accepted indicator of overall cell activity, it lacks important nuanced details regarding cell function. As such further experiments measuring electrophysiological recordings of various basal ganglia regions should be completed. These recordings can provide useful information regarding action potential frequency and strength. The use of calcium or voltage sensitive dye image systems in conjunction with these electrophysiological recordings would provide even more information regarding neuronal health and function.

Finally, additional experimentation should be done investigating any potential role involving CTEP/mGluR5 and synucleinopathy; a key 
pathological feature of PD. Indeed, recent research has shown that $\alpha$ synuclein interacts with cellular prion protein $\left(\mathrm{PrP}^{\mathrm{c}}\right)$ and mGluR5, resulting in cognitive impairment (Ferreira et al., 2017). Furthermore, mGluR5/mTOR activity has strong implications in normal autophagic processes. Indeed, PD related decreases in autophagy has been implicated in Lewy body pathology progression (Tanik et al., 2013). While the data obtained from the 6-OHDA model suggests CTEP would results in an increase in mTOR levels thereby decreasing mTOR related autophagy (potentially exacerbating the already dysfunctional autophagic processes observed in PD), other researcher groups have demonstrated that in models with protein aggregation CTEP actually decreases mTOR in a context dependent manner (Abd-elrahman et al., 2017). Together, the use of CTEP may prove beneficial at clearing toxic protein and reducing PD related cognitive impairments, however admittedly this may come at the expense of CTEPs neuro-recovery potential. To test these hypothesis viral overexpression of mutant $\alpha$ synuclein paired with implanted pre-form fibrils could be used to model Lewy-like pathology (Volpicelli-Daley et al., 2011). Similar to this thesis, the timing of CTEP to begin after $\alpha$-synuclein aggregation has occurred would be extremely important. 


\section{Summary \& Conclusions}

$\mathrm{PD}$ is an age related neurodegenerative disease characterized by degeneration of the dopaminergic nigrostriatal pathway and the accumulation of insoluble protein aggregates known as Lewy bodies (Klockgether, 2004; Factor and Weiner, 2008). The currently available PD therapies merely manage symptom severity and are not able to stop or even slow the neurodegenerative course of the disease. There is a need to focus on stabilization of the neurodegenerative condition via protection of the surviving neurons. There is an additional need to promote neuro-recovery processes. Such processes could maximize the utilization of the existing DA neurons, and/or recruit alternate neuronal pathways to promote recovery by upregulating changes in neuroplasticity. One such avenue is by modulating the glutamatergic mGluR5 GPCR. Indeed, mGluR5 has been previously shown to be implicated in various roles in PD pathogenesis, and as a potential therapeutic target. 
This thesis has demonstrated that targeting mGluR5 with the NAM CTEP is able to reverse the neurodegenerative damage inflicted by the dopaminergic neurotoxin 6-OHDA. Importantly, a clinically relevant model was used throughout the thesis. Specifically, drug therapy occurred only after a lesion sufficient capable of inducing parkinsonian symptoms had been formed. In mice CTEP therapy results in marked improvements in both behaviour and pathology after only 1 week, and full recovery after only 12 weeks. CTEP promoted dopaminergic striatal fibre re-innervation by upregulating the known plasticity promoting factor, mTOR. The upregulation of mTOR also increased striatal levels of the neurotrophic factor BDNF. This thesis showed that mTOR is necessary and required in this model, as blocking mTOR with the antagonist rapamycin prevents any pathological or behavioural improvements imparted by CTEP. These molecular findings are summarized below in (Figure 40). In addition to striatal mTOR, histology and western blot data in other brain regions suggest that CTEP modulates different signaling cascades in different regions of the basal ganglia. This has significant clinical importance as it implies CTEP has the capacity to differentially modulate mGluR5 in context and region-specific manners. 
Unfortunately results from current clinical trials modulating mGluR5 have been mostly disappointing. Similar disappointing results were seen in other conditions (eg. Fragile $\mathrm{X}$ ) that otherwise demonstrated compelling pre-clinical benefit. However, in all current PD-related clinical trials the primary endpoint was targeting the midlate stage therapy related levodopa induced dyskinesia. Its is conceivable that the effectiveness of such a therapy, one that is attempting to promote neuro-recovery and neuroplasticity, would be contingent on early administration.

To date, CTEP has been shown to be a potentially effective therapeutic option in various neurodegenerative disorders including Alzheimer's (Hamilton et al., 2016) and Huntington's disease (Abdelrahman et al., 2017). This thesis adds to the current literature by demonstrating its potential role in treating PD. Furthermore, this thesis is the first to demonstrate that modulation of mGluR5 with a potent negative allosteric modulator can activate neuro-recovery well after neuronal damage has occurred. Regardless of the disease context, this thesis presents data suggesting that modulation of mGluR5 with the 
NAM CTEP may be an effective tool in promoting neuro-recovery through the up-regulation of neurotrophic and neuro-plasticity factors. 


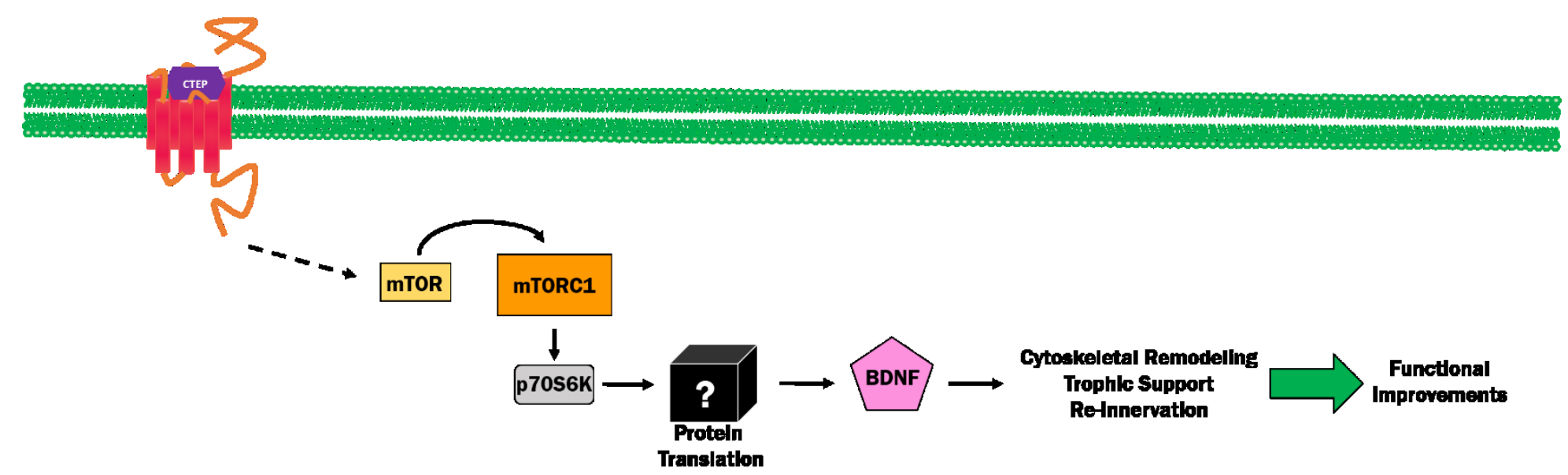

\section{Figure 40 Proposed Mechanism of CTEP action in Striatal DA recovery}

Our data suggests that striatal recovery is due to an increase in mTOR. Specifically, CTEP administration results in phosphorylation of Ser2448 on mTOR proteins. The targeted phosphorylation site paired with the observed increase in activated p70S6K suggests mTORC1 activation. In this model, activation of mTOR is driving protein translation, of which one of the products appears to be BDNF. It is well established that BDNF results in cytoskeletal remodeling and trophic support, and we believe in this case re-innervation. This re-innervation subsequently results in function improvements at the behavioural level. 


\section{Appendix 1}

\section{Preface}

My doctoral work initially started with exploring the use of cytokines as a potential treatment of PD. In my first experiment we tested the efficacy of two peptides, granulocyte macrophage-colony stimulating factor (GM-CSF) and erythropoietin (EPO), in a prodromal/early stage model of Parkinson's disease. The results of this first experiment are outlined below in the peer reviewed published manuscript within Appendix 1b. In brief, we found that both GM-CSF and EPO held neuro-recovery potential, resulting in striatal recovery/reinnervation in rats with a minor lesion. We wished to continue this line of research, however continuing with a rat model was becoming cost prohibitive (specifically due to the cost of the cytokines). Furthermore, our facilities lacked the proper equipment to fully test motor deficiencies in rats. As such we wanted to translate our findings into mice. Rats were initially used as they are the standard animal used with the neuro-toxin 6-OHDA. Before continuing our exploration of cytokines as neuro- 
recovery agents in $\mathrm{PD}$, we wanted to confirm that the repeatedly validated 6-OHDA rat model of PD was indeed translatable into mice our CD1 mice. Appendix 1c outlines our work modelling the early stages of PD using a 6-OHDA mouse model. At this same time our interest were drawn to potential modulation of mGluR5 as a potential therapeutic avenue. As such, while still analyzing the data of our early stage model study we began our first CTEP study outlined in the main thesis. The intention of this study was to investigate the effects of mGluR5 modulation in the early stages of the disease. It was during this experiment that we discovered that something had gone wrong in the study outlined in Appendix 1c, as the animals were demonstrating a significantly more pronounced PD phenotype with respect to apomorphine induced rotations. Additionally, in the original design we had included a GM-CSF treatment group to compare with mGluR5 modulatory therapy (further investigation of the effects of erythropoietin was given to another PhD student in the lab). It was here that we found that if our regular dose of GM-CSF is administered to animals affected by a large lesion, the cytokine treatment is not beneficial and is instead lethal. We suspect the lethality of the cytokine treatment was due to some form of immunological shock. We modified the dosing schedule 
of GM-CSF, however found that the cytokine exacerbated the 6-OHDA induced PD-like pathology and behavioural deficits. The full GM-CSF results of this experiment are outlined in Appendix 1d. As the narrative for GM-CSF was becoming more and more convoluted raising more questions than answering, with the parallel success of the CTEP therapy, we decided to move forward focusing solely on CTEP. 


\section{Appendix 1a \\ Cytokines as novel trophic and immune modulatory treatments for PD}

\section{Introduction}

Immune cells communicate using small glycoproteins known as cytokines, which act in both autocrine and paracrine manners (Caldwell et al., 2014). Cytokines can be grossly defined as either proinflammatory or anti-inflammatory, although many cytokines are in actuality pleiotropic (Litteljohn and Hayley, 2012). By definition, proinflammatory cytokines (e.g. IL-1 $\beta$, TNF- $\alpha$, and IL-6) increase inflammation, and increase immune cell activation and recruitment processes; important components of a healthy immune response immediately after a physical or toxic insult. Conversely, antiinflammatory cytokines (e.g. IL-4, and IL-10) reduce inflammation, and promote the return of immune cells into a resting state and subsequent dispersion or removal of unnecessary or excess immune cells. 
A protracted neural immune response has been found to exacerbate various diseased conditions due to the excessive production of oxidative stress factors (Wadley et al., 2013; Crowley, 2014). Importantly, it seems that during chronic immune system activation, macrophage cells often become "stuck" in an autocrine loop, causing the immune cells to remain continually in a pro-inflammatory state. Additionally, with a prolonged immune response there is often not only an increase in pro-inflammatory signalling, but also an increase in cellular autophagy and concomitant increase in cytosolic debris (Ma et al., 2013). The accumulation of cellular waste products exacerbates the toxic environment promoting a further increase in immune system activation. Indeed, neuro-inflammation has been shown to contribute to PD pathology. Our lab has demonstrated that early life exposure to the bacterial endotoxin lipopolysaccharide (LPS) is able to enhance the neurodegenerative effects of paraquat to induce PD like pathology in mice (Mangano and Hayley, 2009; Mangano et al., 2011). This toxin is known to induce an increase of pro-inflammatory cytokines, even resulting in permanently elevated levels of various inflammatory factors 
in adult mice that were prenatally exposed to LPS (Ling et al., 2006; Barlow et al., 2007).

The use of cytokines as therapeutic agents initially started within the domain of cancer, as a main symptom of invasive cancers is a systemic high-jacking of the immune system to protect the developing malignancy. Interestingly, many of the same pathways that are implicated in oncogenesis and uncontrolled cancer growth are the same pathways implicated in pre-clinical regeneration studies. As such researchers started looking at cytokines for the use in regenerative medicine. Two cytokines that may hold potential therapeutic significance are the hematopoietic cytokines, erythropoietin (EPO) and granulocyte macrophage-colony stimulating factor (GM-CSF). Indeed, GM- CSF has protective effects in models of Alzheimer's disease (Boyd et al., 2010), and in MPTP and paraquat models of PD (Kim et al., 2009a; Mangano et al., 2011). Moreover, GM-CSF administration induced spontaneous axonal regeneration and functional recovery in models of traumatic spinal cord injury (Ha et al., 2005; Bouhy et al., 2006) and reduced infarct volume following ischemic stroke (Schäbitz et al., 2008; Nakagawa et al., 2014). Similarly, EPO has been 
investigated extensively for use in stroke, traumatic head injury and more recently, in toxin based animal models of PD (Sargin et al., 2010; Bond and Rex, 2014; Merelli et al., 2014). EPO was also shown to protect hippocampal neurons from stressor-induced apoptosis, and increased adult hippocampal neurogenesis (Merelli et al., 2014).

GM-CSF and EPO have well-documented trophic actions in the periphery and can infiltrate and accumulate within the brain (Enzler and Dranoff, 2003). Receptors for GM-CSF and EPO have been found on mature DA neurons and neural progenitor cells, suggesting that they might influence adult neuronal functioning, as well as stimulate maturation (Kim et al., 2004; Ha et al., 2005). Indeed both GM-CSF and EPO are Type I cytokines, and therefore their receptors are composed predominantly of multimeric chains and signal through Jak/STAT related pathways (Lee and Margolin, 2011). The peripheral function of GM-CSF is to promote the differentiation and maturation of innate immune cells, and it is routinely administered to cancer patients to modify neutrophil production (Dale et al., 1998). Similarly, EPO has potent mitogenic effects on immune cells, as well as red blood cells and is routinely prescribed for anemia and in the context of certain cancer 
treatments (Debeljak et al., 2014). Thus, both GM-CSF and EPO have well established clinical records. Mechanistically, these cytokines are potent inducers of BDNF and GDNF (Bouhy et al., 2006; Mengozzi et al., 2012), which is believed to be fundamental for their beneficial neural consequences. Finally, while EPO possess predominantly antiinflammatory and growth factor related mechanisms of action (Han et al., 2011), GM-CSF is known to be pleiotropic and imparts both antiapoptotic/neurotrophic actions (Bouhy et al., 2006; Kim et al., 2009a) but can also initiate and mobilize immune related cells and proinflammatory signalling cascades (Henze et al., 2005; Bernasconi et al., 2010; Parajuli et al., 2012). As such, continued pre-clinical investigations are required to determine the suitability of both EPO and GM-CSF as potential translational treatments of the human condition. 


\title{
Appendix 1b
}

\section{Hematopoietic cytokines as therapeutic players in early stages Parkinson's disease}

\author{
K. Farmer, C.R. Rudyk, N.A. Prowse, \& S.P. Hayley.
} Presented as published.

Frontiers in Aging Neuroscience, 2015

DOI: $10.3389 /$ fnagi.2015.00126

PMID: 26191001

\begin{abstract}
Parkinson's disease (PD) is a devastating age related neurodegenerative disease that is believed to have a lengthy prodromal state. It is critical to find methods of interfering with the progression of this early degenerative stage by inducing compensatory recovery processes to slow or prevent the eventual clinical symptoms. The current perspective article argues that immune system signalling molecules represent such a promising therapeutic approach. Two cytokines of interest are granulocyte macrophage-colony stimulating factor (GM-
\end{abstract}


CSF) and erythropoietin (EPO). These hematopoietic cytokines have been protective in models of stroke, neuronal injury, and more recently PD. It is our belief that these trophic cytokines can be used not only for cell protection but also regeneration. However, success is likely dependent on early intervention. This paper will outline our perspective on the development of novel trophic recovery treatments for PD. In particular, we present new data from our lab suggesting that EPO and GM-CSF can foster neural re-innervation in a "mild" or partial lesion PD model that could be envisioned as reflecting the early stages of the disease.

\section{Parkinson's disease: Animal model of early stages}

Parkinson's disease (PD) is characterized by a loss of dopamine (DA) neurons within the nigrostriatal pathway and the presence of Lewy body pathological protein aggregates (Farrer et al., 2001; Sherer et al., 2001). Clinically, PD is diagnosed based on tremors within distal limbs, muscle rigidity, and bradykinesia. By the time patients present with these motor symptoms, there has already been significant degeneration of DA neurons, with up to an $80 \%$ loss of striatal DA innervation (Bernheimer et al., 1973; Bezard et al., 2001). There are also extensive 160 
non-motor symptoms, which present long before the cardinal motor symptoms (McDonald et al., 2003; Gökbayrak et al., 2014).

Current PD treatments only manage symptom severity and are not able to reverse or even appreciably slow the neurodegenerative processes. Thus, it is of interest to investigate potential treatments that could stabilize these surviving neurons and possibly induce some degree of neuronal recovery. It might be advantageous to target processes linked to the early or prodromal stages of PD, as neuronal plasticity would likely be more amenable to modulation at such times. However, models of early stage PD are less common and not as well understood as the late stage models typically used.

The neurotoxin, 6-hydroxydopamine (6-OHDA), is routinely used to induce PD-like pathology, inducing a loss of substantia nigra pars compacta $(\mathrm{SNc}) \mathrm{DA}$ neurons and downstream striatal terminals (Kirik et al., 1998; Alvarez-Fischer et al., 2008). 6-OHDA infused directly into the SNc rapidly produces a robust degeneration of SNc DA neurons, coupled with striatal DA depletion within 48-72 hours (Blandini et al., 2008; Thiele et al., 2012). However, this method has the obvious caveat of not reflecting the chronic slow course of degeneration. 
A more progressive lesion has been observed with lower doses of 6OHDA infused into the striatum rather than SNc. Indeed, intra-striatal 6-OHDA administration induced a lesion, which gradually increased in size over several weeks (Sauer and Oertel, 1994; Blandini et al., 2008) and more closely mimicked the progression from early to later stages of PD.

\section{Novel treatment strategies}

One exciting new avenue for treating PD involves the use of trophic factors to stabilize neuronal viability and even promote some degree of recovery. In fact, recent studies have revealed a reduction of brain derived neurotrophic factor (BDNF) within the SNc of PD patients (Mogi et al., 1999; Salehi and Mashayekhi, 2009). Accordingly, BDNF can promote the survival and differentiation of mesencephalic DA neurons, as well as protect against the DA toxicants, MPTP and 6OHDA (Murer et al., 2001). Likewise, glial derived neurotrophic factor (GDNF), has also emerged as a potential candidate for neuroprotection in PD patients, based on success in various animal models (Gash et al.,

1998; Fox et al., 2001; Ai et al., 2003). However, the improvements 
observed in clinical trials were restricted to the immediate area surrounding the site of infusion (Gill et al., 2003; Love et al., 2005) and a randomized placebo-controlled study was unsuccessful at replicating these beneficial effects (Lang et al., 2006). Moreover, BDNF and GDNF do not readily cross the blood brain barrier $(\mathrm{BBB})$ and have numerous side effects (Pezet and McMahon, 2006; Pilakka-Kanthikeel et al., 2013).

Finding well-tolerated factors with trophic properties, which cross the BBB represents a considerable challenge. Two cytokines that may hold potential therapeutic significance are the hematopoietic cytokines, erythropoietin (EPO) and granulocyte macrophage-colony stimulating factor (GM-CSF). Indeed, GM-CSF had protective effects in models of Alzheimer's disease (Boyd et al., 2010), and in MPTP and paraquat models of PD (Kim et al., 2009a; Mangano et al., 2011). Moreover, GM-CSF administration induced spontaneous axonal regeneration and functional recovery from traumatic spinal cord injury (Ha et al., 2005; Bouhy et al., 2006; Huang et al., 2007) and reduced infarct volume following ischemia (Nakagawa et al., 2006; Schäbitz et al., 2008). Similarly, EPO has been investigated extensively for use in 
stroke, traumatic head injury and more recently, in toxin based animal models of PD (Sargin et al., 2010; Merelli et al., 2013; Bond and Rex, 2014). EPO was also shown to protect hippocampal neurons from stressor-induced apoptosis, and increased adult hippocampal neurogenesis (Merelli et al., 2015).

GM-CSF and EPO have well-documented trophic actions in the periphery and can infiltrate and accumulate within the brain (McLay et al., 1997; Enzler and Dranoff, 2003; Kasper, 2003). Receptors for GMCSF and EPO have been found on mature dopamine neurons and neural progenitor cells, suggesting that they might influence adult neuronal functioning, as well as stimulate maturation (Kim et al., 2004; Ha et al., 2005).

The peripheral function of GM-CSF is to promote the differentiation and maturation of innate immune cells, and it is routinely administered to cancer patients to modify neutrophil production (Dale et al., 1998). Similarly, EPO has potent mitogenic effects on immune cells, as well as red blood cells and is routinely prescribed for anemia and in the context of certain cancer treatments (Debeljak et al., 2014). Thus, both also have well established clinical track records. 


\section{GM-CSF and EPO promote striatal re-innervation}

It is thought that some critical threshold of neuronal loss must be reached before clinical PD pathology is manifested. Accordingly, a slow progressive "wave" of neurodegeneration may spread through the nigrostriatal tract over many years or decades. Accordingly, it is of interest to study the effects of potential treatments while the disease is still in an early or possibly even a prodromal stage. In the present paper, it was of interest to assess whether EPO or GM-CSF treatment could influence striatal innervation following the establishment of a partial or "mild" lesion that might be analogous to the early onset of the disease.

As shown in Figure 41 , we did indeed find that a modest $(\sim 10 \%$ of striatal area, as determined using TH staining) lesion was induced in rats following intra-striatal infusion of a single moderate dose of 6-OHDA $(20 \mu \mathrm{g})$. However, no significant neuronal loss was evident within the SNc using this relatively mild paradigm, although it is possible that some of these SNc neurons would eventually die if we explored longer time intervals. Indeed, many surviving neurons had irregular shaped soma (Figure 42). 
Importantly, both EPO (50 $\mu \mathrm{g} / \mathrm{kg}$; ip; two injections) or GMCSF (10 $\mu \mathrm{g} / \mathrm{kg}$; ip; two injections) treatments given after the presumed establishment of the lesion (i.e. on Days 13 and 28 following 6-OHDA) provoked a significant recovery (presumed re-innervation) of striatal terminals. The statistical analysis revealed a significant Treatment effect, $\mathrm{F}(2,15)=10.7, \mathrm{P}<0.01$, with regards to striatal lesion size (Figure 41). Specifically, GM-CSF and EPO administration prevented the striatal lesion by Day 30 following 6-OHDA.

The present findings are consistent with our own previous data and those of others showing beneficial effects of GM-CSF (Mangano et al., 2011; Kosloski et al., 2013) and EPO (Xue et al., 2007; Dhanushkodi et al., 2013; Qi et al., 2014) in toxicant based PD models. However, since GM-CSF or EPO were administered after lesion establishment, the effects of the cytokine treatments in the current study would be expected to reflect some degree of recovery involving DA fibre re-growth rather than the prevention of fibre loss in the first place. This is a particularly novel finding given that the majority of studies typically focus on neuroprotective effects, rather than addressing the more clinically 
relevant issue of promoting recovery following some degree of neuronal damage.

\section{Impact and future directions}

It is important to underscore that the 6-OHDA paradigm presently used provoked a very modest loss of striatal terminals and future studies are required to ascertain whether GM-CSF and EPO might also have reparative properties in PD models with more significantly sized lesions. Nonetheless, GM-CSF and EPO may be ideal trophic treatment candidates based on their biological profiles, preclinical data and track record of clinical applicability. Mechanistically, these cytokines are potent inducers of BDNF and GDNF (Bouhy et al., 2006; Mengozzi et al., 2012), which we posit to be fundamental for their beneficial neural consequences. Targeting trophic processes to boost plasticity may be a critically important shift in treatment modalities away from failed attempts to translate neuroprotective approaches to the clinic; this strategy would also work well in tandem with recent efforts to identify biomarkers of disease state. 


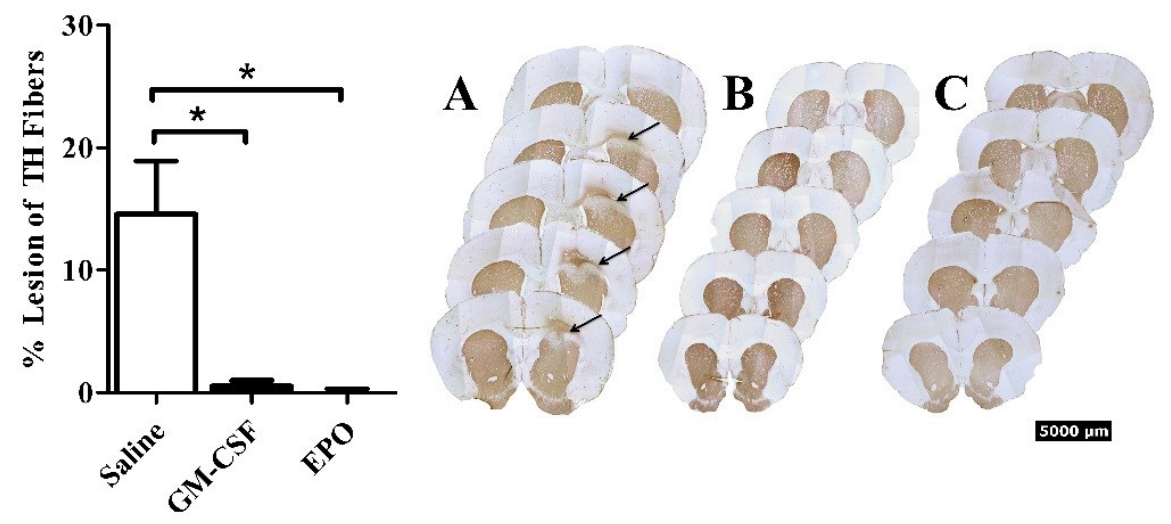

Figure 41 Appendix 1b - Striatal damage.

Thirty days after the intra-striatal infusion of 6-OHDA, (A) saline treated animals had a modest but statistically significant loss of $\mathrm{TH}+$ fibres. The (B) GM-CSF and (C) EPO treated animals displayed no visible lesion at the 30 -day time point. Data is expressed as mean \pm 1 SEM, $* p<0.01$. 


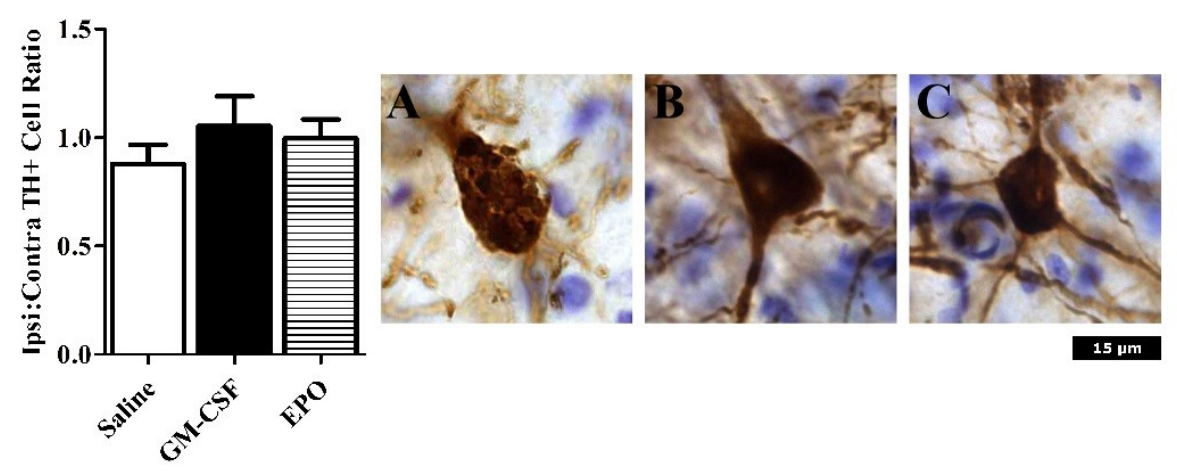

Figure 42 Appendix 1b-Stereological count of the $\mathrm{SNc}$

Neurons were counted in an unbiased manner using MBF Stereo investigator optical fractionator probe. There were no significant differences between treatment groups (left graph). Photomicrographs are representative of animals treated with (A) Saline; (B) GM-CSF; or (C) EPO in conjunction with 6-OHDA infusion. The saline (but not GM$\mathrm{CSF}$ or EPO) treated rats that received 6-OHDA displayed $\mathrm{TH}+$ neurons with an abnormal shaped nucleus, with a reduction of projections. Images were taken at 40x magnification. 


\section{Additional findings not included in manuscript}

\section{Additional time points}

In addition to day 30 , we investigated the effects of cytokine treatment at day 15. At this time point we found no difference in $\mathrm{TH}+$ cells within the substantia nigra when counted using stereological methods (Figure 43). We did however find that GM-CSF exacerbated the effects of 6-OHDA, increasing striatal lesions to an average $21.2 \%$ of area compared to $9.4 \%$ in saline treated animals $(\mathrm{p}<.05)$ (Figure 44$)$.

\section{Microglial activation}

An isolectin B4-FITC conjugate histochemical stain was used on striatal tissue to label microglia, on every $10^{\text {th }}$ section of the striatum between coordinates $1.20 \mathrm{~mm}$ and $-0.70 \mathrm{~mm}$, and every $6^{\text {th }}$ slice of the substantia nigra between the coordinates $-4.80 \mathrm{~mm}$ and $-6.30 \mathrm{~mm}$. Isolectin B4-FITC conjugate $(2.5 \mu \mathrm{g} / \mathrm{mL})$ was dissolved in $10 \mathrm{mM}$ PBS with $2 \%$ Triton-X, which was allowed to incubate at room temperature 
overnight for 18 hours. Isolectin B4 is a marker of microglia that can be used to label varying states of microglial activation.

A qualitative serial scan of striatal tissue sections revealed increased levels of isolectin B4 positive staining in GM-CSF treated rats at the 15-day time, relative to saline injected controls (Figure 45). In contrast, at this time, EPO treatment reduced levels of the microglial marker, compared to saline treatment. However, at the 30-day time, isolectin B4 staining was substantially reduced overall to such a degree compared to earlier interval that no differences were evident between the treatment groups. 


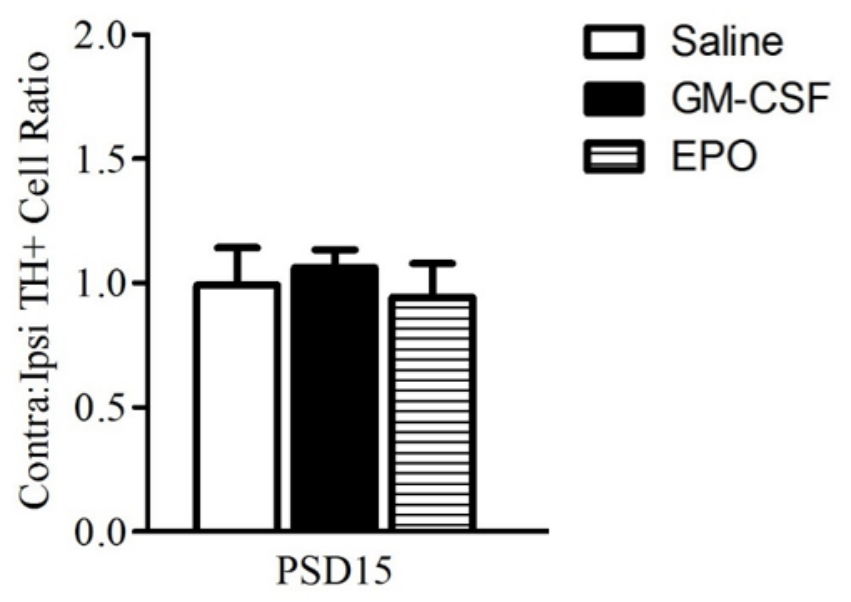

Figure 43 Appendix 1b-Stereological count of the SNc at Day 15

We found no difference in total number of $\mathrm{TH}+$ cells within the substantia nigra between treatment groups 15 days after 6-OHDA administration. 

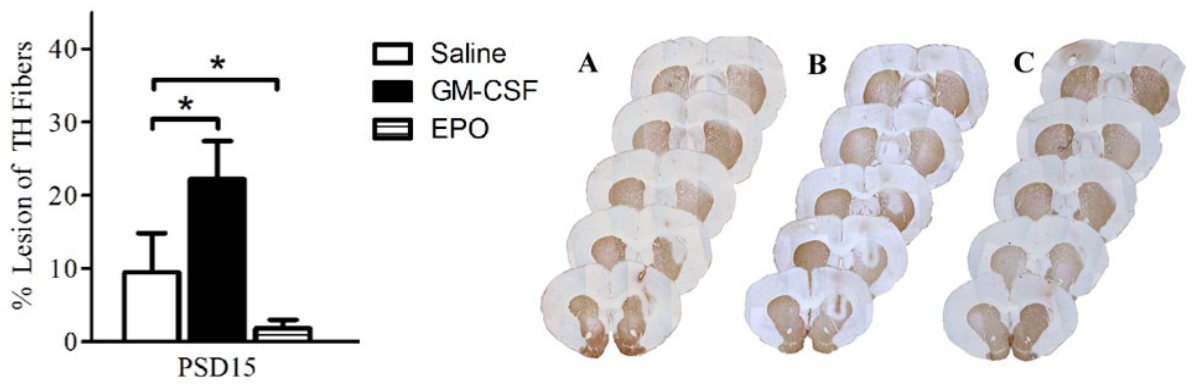

Figure 44 Appendix 1b - Striatal damage at Day 15

Fifteen days after the intra-striatal infusion of 6-OHDA, (A) saline treated animals had a modest striatal lesion. The (B) GM-CSF treated animals displayed an increase in striatal damage, compared to the saline treated animals, while (C) EPO treated animals displayed a reduced lesion compared to saline controls. Data is expressed as mean $\pm 1 \mathrm{SEM}$, $* \mathrm{p}<.05$. 

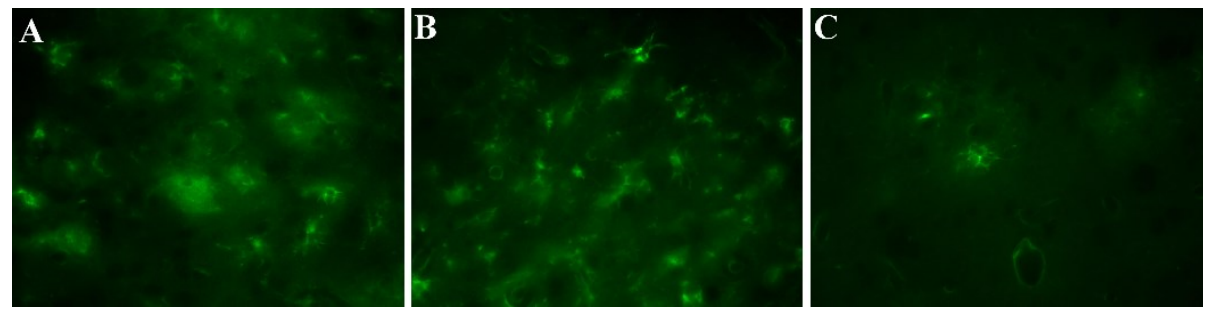

\section{Figure 45 Appendix 1b - Microglial Activation at Day 15}

The GM-CSF treated animals B) displayed elevated isolectin B4-FITC staining, relative to rats that received saline A). Moreover, the morphology of the microglial cells suggested an intermediately activated state. In contrast, EPO treated animals C) showed minimal positive staining for the microglial marker. Similarly, at the 30-day time following 6-OHDA, there were only a few scattered isolectin B4-FITC labelled cells and no observable differences were evident between the treatment groups. 


\section{Discussion on additional findings}

It was surprising that the effects of GM-CSF treatment varied so dramatically depending upon the time of administration. Indeed, the cytokine increased striatal damage at the 15-day time point, but reduced damage 30 days following 6-OHDA. We hypothesize that this differential effect is related to inflammatory state of the microenvironment in which the cytokine acts. In this regard, the phenotype of microglia has been reported to vary between the so called M1 state, wherein they release a variety of pro-inflammatory and prooxidative factors; to the M2 state, which is characterized by the release of anti-inflammatory and protective anti-oxidant factors (Hu et al., 2012). Along these lines, our present analyses revealed that microglia appeared to be activated by GM-CSF at the 15-day but not the 30-day time; whereas EPO reduced microglial staining at both times. Thus, the microglial phenotype/activation state appear to be temporally linked to the overall influence of the cytokine treatment on striatal $\mathrm{TH}+$ fibre density. The isolectin B4 data is also consistent with reports that 6OHDA provokes the activation of microglia in the first two weeks (Walsh et al., 2011), however activation is significantly reduced four weeks after administration of the neurotoxin (Blandini et al., 2008). 
Similarly, elevated microglia reactivity has been reported within the first 14 days, with a return to basal levels within 30 days following traumatic striatal injury (Batchelor et al., 1999) or ischemic stroke (Lehrmann et al., 1997). In effect, GM-CSF might have synergized with pro-death pathways when administered in the context of an inflammatory microenvironment, however may have the opposite effect at later times when most inflammatory cascades have resolved.

These items were omitted from the original manuscript at the reviewers request, as they felt the isolectin histology was not optimal. 


\section{Appendix 1c Development of an early stage model of Parkinson's disease}

\section{Introduction}

Initially the intention of this thesis was to examine the effects of potential therapeutics in a prodromal model - a model where there is sizeable neuropathology however is lacking in any overt behavioural phenotype. As no such model existed we aimed to generate one using low doses of intra-striatal 6-OHDA. Building on our previous findings described in Appendix 1b we wanted to further examine the individual "sick cell" phenotype - where cells had decrease projections (both number and thickness) and increased vacuolization. We believed that these phenotypic differences we observed in Appendix 1 may be as simple as DA neurons that were in direct contact with 6-OHDA versus DA neurons that were not. We believed that perhaps cells whose fibres were near the injection site would display the sick phenotype, as they would be in direct contact with the neurotoxin. Conversely, we believed 
cells that were more indirectly affected by the microenvironment rather than direct toxin administration would appear comparatively healthier. Additionally, we wanted to see if microglia would mobilize and surround neurons in direct contact with the toxin, or if they would simply mobilize to the general SNc region. To test this, we permanently affixed the cannula to the skull of the mice and gave an injection of Fluorogold 1 week prior to the 6-OHDA injection - thereby labelling all the cells within the SNc that had projections within the injection site.

\section{Methods}

Animals

Male CD1 mice aged 6-8 weeks on arrival were used. Animals were individually housed in a standard polypropylene cage and maintained a 12-hour light/dark cycle. Tap water and food (Tekland 2014 Mouse Chow) was provided ad libitum, while room temperature and humidity were maintained at $20^{\circ} \mathrm{C}$ and $50 \%$, respectively. All aspects of this experiment were approved by the Carleton University Committee for Animal Care and adhered to the guidelines outlined by the Canadian Council for the Use and Care of Animals in Research. 


\section{Surgery}

The mice were anaesthetized using isoflurane inhalational anaesthetic $(2-3 \%$ in pure $\mathrm{O} 2)$. Animals were placed in a Kopf instruments stereotaxic frame, then a $3 \mathrm{~mm}$ guide cannula and a single support screw were secured using Dentsply dental acrylic in the right anterior dorsal striatum (cannula at the coordinates $1.75 \mathrm{~mm} \mathrm{M} / \mathrm{L}$, $0.90 \mathrm{~mm} \mathrm{~A} / \mathrm{P}$, and $3.00 \mathrm{~mm} \mathrm{D} / \mathrm{V}$, with respect to bregma, and the support screw $3.00 \mathrm{~mm}$ posterior to the cannula). A single infusion of $2 \%$ Fluorogold solution in $0.9 \%$ sodium chloride was administered at a rate of $0.4 \mu \mathrm{L} / \mathrm{min}$ using a Hamilton $10 \mu \mathrm{L}$ syringe attached to a Harvard Apparatus Pico Plus pump. After the infusion the injector was allowed to rest for 5 minutes before being retracted. The guide cannula was capped with a dummy cap, and animals sutured using absorbable sutures.

\section{Infusion}

Seven days after cannulation mice were anaesthetized again using isoflurane inhalational anaesthetic $(2-3 \%$ in pure $\mathrm{O} 2)$. The dummy 
cap was then removed, followed by an infusion as described above of either $0,2,4,12$, or $20 \mu \mathrm{g}$ of 6-hydroxydopamine hydrochloride (Sigma Aldrich, $\mathrm{H} 4381$ ) in $0.02 \%$ ascorbic acid in $0.9 \%$ sodium chloride solution.

\section{Behaviour}

A time-course of events can be found in Figure 46.

\section{Catwalk XT}

Animals were trained on the Noldus Catwalk XT system for 4 days, wherein they had to complete 3 compliant runs within 5 minutes. A compliant run consists of a run with a duration between $0.5-5$ seconds with no stopping in the middle of the CatWalk system. Animals that were not successful in the 5 minute trial were given a 30 minute break followed by a re-test until successful completion of the training protocol. Animals were tested on the CatWalk before surgery (pre surgical baseline), before administration of 6-OHDA (pre-6OHDA baseline), and every 10 days following 6-OHDA administration for 30 days total. 
Note: due to the staggering of treatment groups and an error in machine calibration, the data from the $20 \mu \mathrm{g}$ of 6-OHDA group has been omitted.

\section{Rota Rod}

Animals were trained on the rota rod for 2 days as previously described (Heuer, et al. 2012). Day 3 was the test day and occurred 30D after administration of the neurotoxin. On the test day mice were tested three times on an accelerating protocol in which the rotation speed was increased from 4 to 88 RPM over 600 seconds. Each time the animal fell of the apparatus would mark the end of one trial. Trials were run in triplicate with a 1 hour break in between each trial. The lowest of the 3 trials was discarded, and the average of the remaining two trials was used as the animals score.

\section{Home-cage locomotion}

Animals home-cage locomotion was recorded using an infrared beam break apparatus (Micromax: Accuscan Instruments, Columbus OH, USA). Locomotor activity was measured for 12 hours during the 8pm-8am light cycle between days 29 and 30 of the experimental time- 
course. Animals were given 4 hours to acclimatize to the room prior to assessment. Locomotor activity was recorded as the total number of beam breaks during the $12 \mathrm{hr}$ test period.

\section{Apomorphine induced rotations}

On Day 30 following the infusion of 6-OHDA, animals received $0.5 \mathrm{mg} / \mathrm{kg}$ subcutaneous injection of Apomorphine hydrochloride hemihydrate $(0.1 \mathrm{mg} / \mathrm{mL}$ in $0.9 \%$ sodium chloride). Animal tracks were recorded using the Noldus Ethovision tracking camera. The number and direction of the rotations were recorded for 20 minutes, following a 5 minute drug incubation period. The number of net contra lateral rotations are reported.

\section{Euthanization}

A subset of animals were euthanized 60 minutes after the injection of apomorphine via rapid decapitation. Of these animals the striatum and substantia nigra were extracted for HPLC and Western Blot

analysis, respectively. Another subset of animals were euthanized on Day 30 via transcardial perfusion with $4 \%$ paraformaldehyde solution 
following an anesthetic overdose of sodium pentobarbital. Tissue was post fixed for $24 \mathrm{hrs}$ in the same solution, followed by washes of increasing concentration of sucrose buffered solution. Tissue was allowed to rest for $72 \mathrm{hrs}$ in a $30 \%$ sucrose solution, then flash frozen and stored at $-80 \mathrm{oC}$ until ready to be process. This tissue was extracted for immunohistochemical analysis.

\section{HPLC - DA, DOPAC, and HVA concentrations}

Levels of DA and its metabolites, DOPAC and HVA, were measured using high performance liquid chromatography as previously described (Anisman \& Zacharko, 1990). The presented data is expressed as a ratio of the contralateral to ipsilateral sides.

\section{Autophagy}

Autophagy was measured 30 days after administration of 6OHDA by quantifying the conversion of LC3-I to LC3-II using Western Blot Analysis. Briefly, $2 \mu \mathrm{g}$ of crude protein extract was separated in a 15\% polyacrylamide gel. Rabbit anti-human MAP1LC3A/B (AbD Serotec; AHP2167) was used at a concentration of 1:16,000 with a goat 
anti-rabbit IgG-HRP (Santa Cruz Biotechnology; SC-2004) secondary being used at 1:5,000. All bands were normalized to $\beta$-actin-HRP (1:20,000; Santa Cruz Biotechnology; SC-47778) and their integrated density values were measured using AlphaEase FC software (v 3.1.2). Values are expressed as a ratio of LC3-II to LC3-I.

\section{Statistics}

One-way ANOVAs were completed as appropriate. Post hoc testing was completed using Fisher's PLSD. 


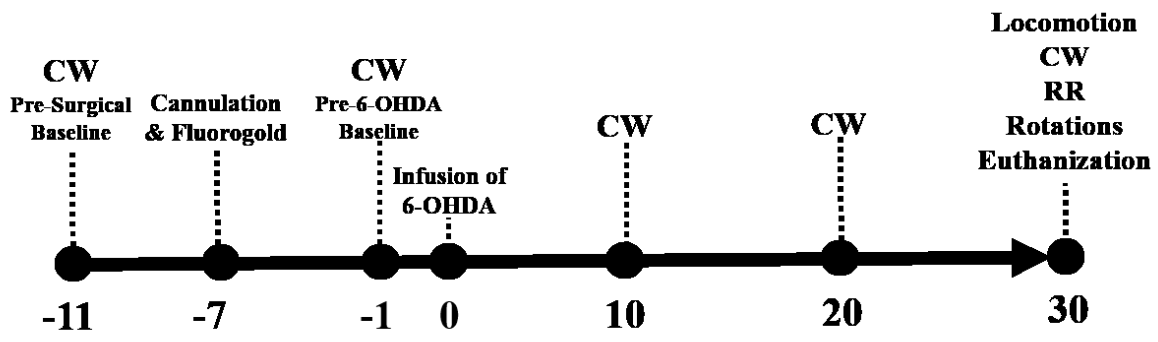

Time

Figure 46 Appendix 1c - Prodromal model time course

This figure represents the schedule and timing of procedures for the development of an early stage model of Parkinson's disease study. 


\section{Results}

\section{Behaviours}

Thirty days following administration of 6-OHDA animals were tested in with various behaviours. Extensive gait analysis was done using the CatWalk XT system. At baseline there was no statistical difference between animals. After 6-OHDA administration, there was a significant reduction in paw area and swing duration for left side paws. No other differences in the animals gait were identified (Table 33). Following a 12-hour dark cycle micromax recording, a one way ANOVA found a significant treatment effect $(\mathrm{F}(4,45)=3.315, \mathrm{p}=$ .0183) (Figure 47). A Fishers PLSD post hoc analysis found the $20 \mu \mathrm{g}$ had significantly more activity compared to all other treatment groups $(\mathrm{p}<.05)$. When tested on the accelerating rota rod a one way ANOVA found a significant treatment effect $(F(4,50)=4.007, p=.0068)$ (Figure 48). Follow up comparisons found that the $20 \mu \mathrm{g}$ group was significantly impaired compared to all other treatment groups $(\mathrm{p}<.05)$. A one-way ANOVA found a significant treatment effect $(\mathrm{F}(4,35)=4.774, \mathrm{p}=$ $.0035)$, when comparing the number of contralateral rotations following an injection of apomorphine (Figure 49). Post hoc comparisons found 
the $20 \mu \mathrm{g}$ group had significantly more rotations compared to the 0,2 , $4 \mu \mathrm{g}$ groups $(\mathrm{p}<.05)$. 


\section{Table 3 Appendix 1c - Summary of Catwalk Data}

\begin{tabular}{|c|c|c|}
\hline Parameter & Time-point & Results \\
\hline All & Baseline & $\begin{array}{l}\text { No significant difference between } \\
\text { animals }\end{array}$ \\
\hline Paw Area & Time-course & Left side has reduced paw area $(p<.05)$ \\
\hline Swing duration & Time-course & $\begin{array}{l}\text { Left side has reduced swing duration }(p< \\
.05)\end{array}$ \\
\hline Swing Speed & Time-course & No significance \\
\hline Stride length & Time-course & No significance \\
\hline RegIndex & Time-course & No significance \\
\hline BOS & Time-course & No significance \\
\hline
\end{tabular}

Area: max contact area of each paw.

Swing duration: the time in which the animals paw is not in contact with the floor.

Swing speed: the speed of the paw while in the air (difference in time and distance between successive paw placements).

Stride length: the distance between successive placements of the same paw.

RegIndex: the number of normal step sequence patterns relative to the total number of paw placements (expressed as a \%).

Base of Support (BOS): the average width between either the front limbs or hind limbs, respectively. 


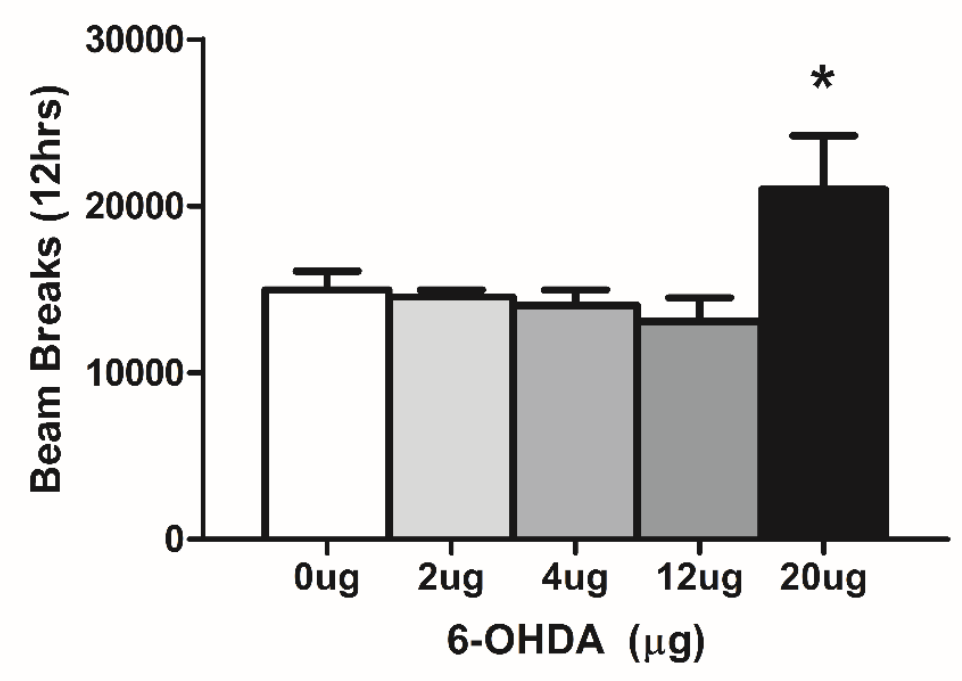

Figure 47 Appendix 1c - Micromax

Total locomotion was recorded overnight during a $12 \mathrm{hr}$ period on day 29/30 of the experiment using the MicroMax IR beam system. A one way ANOVA found a significant treatment effect $(F(4,45)=3.315, p=$ $.0183)$. A Fishers PLSD post hoc analysis finds differences between the $20 \mu \mathrm{g}$ and all other treatment groups $(\mathrm{p}<.05)$. 


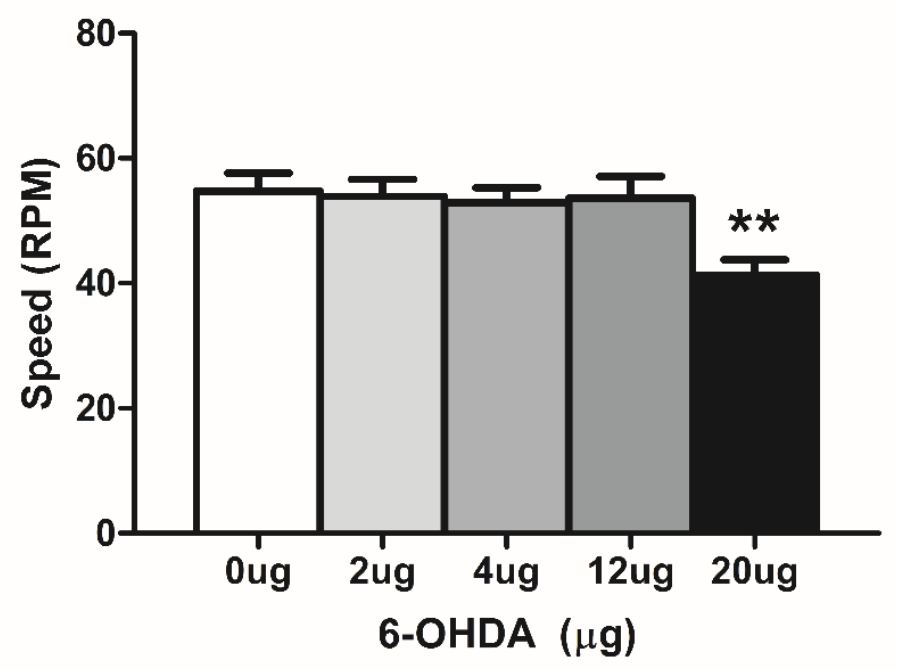

Figure 48 Appendix 1c - Rota Rod

Animals were tested on the accelerating Rota Rod on day 30 of the experiment. A one way ANOVA found a significant treatment effect $(\mathrm{F}(4,50)=4.007, \mathrm{p}=.0068)$. Follow up comparisons found a significant difference between the $20 \mu \mathrm{g}$ group and all other treatment groups $(\mathrm{p}<$ $.05)$. 


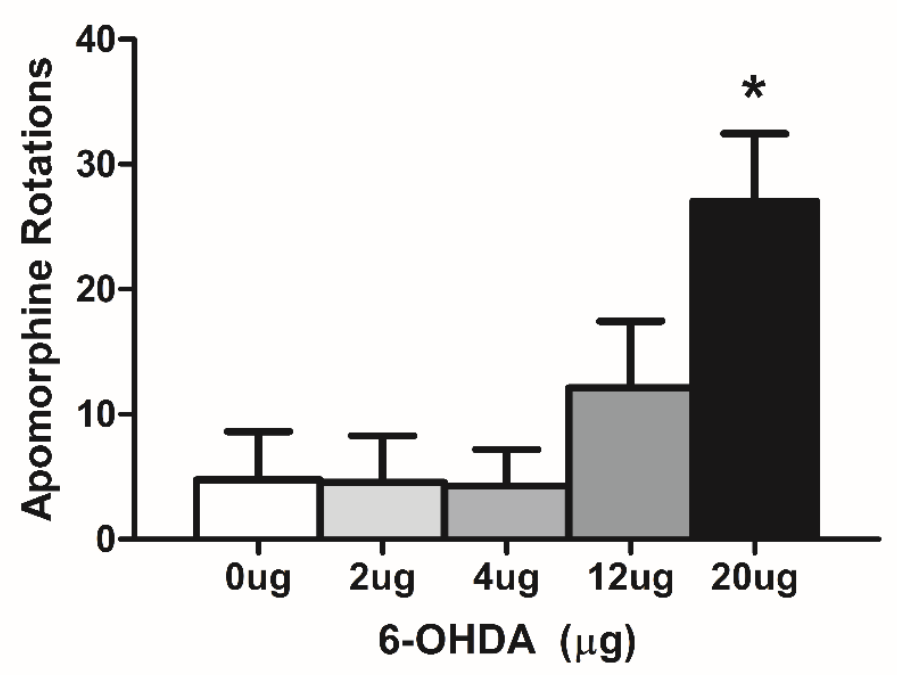

Figure 49 Appendix 1c - Apomorphine induced rotations

Animals were given injections of apomorphine 30 days after administration. The net contra-lateral rotations over a 20 minute period were recorded. A one-way ANOVA found a significant treatment effect $(\mathrm{F}(4,35)=4.774, \mathrm{p}=.0035)$. Post hoc analysis found a significant difference between the $20 \mu \mathrm{g}$ group and the $0,2,4 \mu \mathrm{g}$ groups $(\mathrm{p}<.05)$. 


\section{Molecular}

Striatal tissue was analyzed using HPLC for dopamine and dopamine metabolites. A one way ANOVA found a significant treatment effect $(\mathrm{F}(4,33)=6.391, \mathrm{p}=.0006)$ when comparing striatal dopamine levels (Figure 50). Follow up comparisons found significant decrease in dopamine between the $0 \mu \mathrm{g}$ group and all other treatments $(\mathrm{p}$ $<.05)$. Similarly, there was a significant difference between the $20 \mu \mathrm{g}$ and all other treatments $(\mathrm{p}<.05)$. When comparing the dopamine metabolite DOPAC, a one way ANOVA found a significant treatment effect $(\mathrm{F}(4,33)=4.028, \mathrm{p}=.0091)$ (Figure 51). A post hoc analysis found that the 12 and $20 \mu \mathrm{g}$ groups had significantly less DOPAC compared to sham controls $(\mathrm{p}<.05)$. When comparing HVA levels, a one way ANOVA find no significant treatment effect $(\mathrm{F}(4,33)=1.348$, $\mathrm{p}=.2731)($ Figure 52).

The amount of LC3 I/II, and indicator of autophagic processes, was measured in striatal tissue lysates by western blot. A one way ANOVA found a significant treatment effect $(\mathrm{F}(4,6)=105.237, \mathrm{p}<$ .0001) when comparing between groups (Figure 53). The follow up comparisons found a significant difference that the $0 \mu \mathrm{g}$ group had 
significantly lower LC3 I/II levels compared to all other treatments $(\mathrm{p}<$ $.05)$. Conversely, $20 \mu \mathrm{g}$ treated animals had significantly higher levels of LC3 I/II compared to all other treatment groups $(\mathrm{p}<.05)$. 


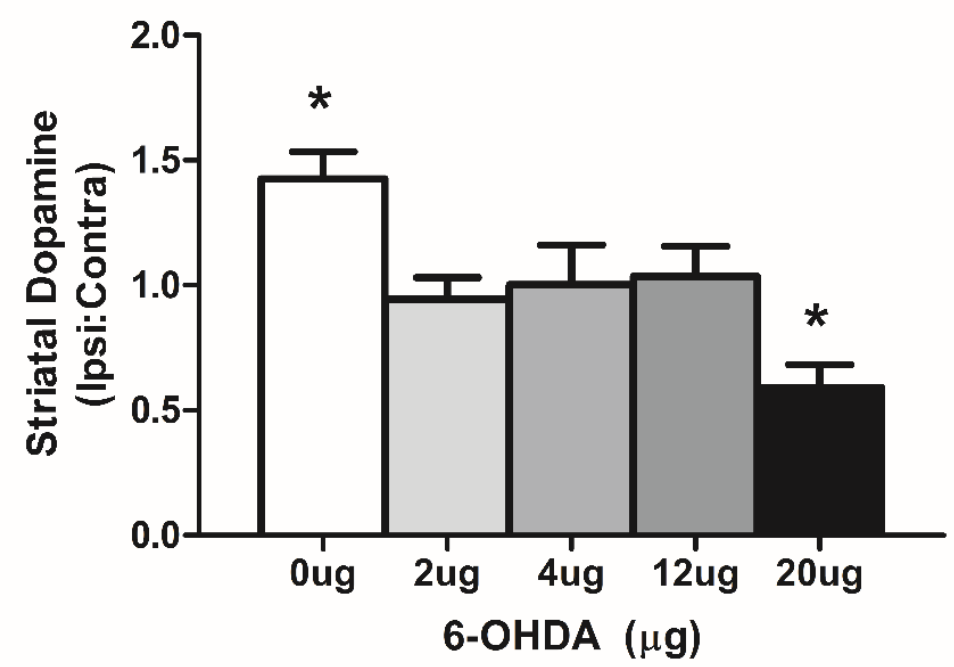

Figure 50 Appendix 1c - HPLC Striatal Dopamine

Striatal dopamine levels at day 30 was measured using HPLC. A one way ANOVA found a significant treatment effect $(\mathrm{F}(4,33)=6.391, \mathrm{p}=$ .0006). Follow up comparisons found that the $0 \mu \mathrm{g}$ group had significantly higher levels of dopamine compared to all other treatments $(\mathrm{p}<.05)$. Similarly, the $20 \mu \mathrm{g}$ group had significantly lower striatal dopamine levels compared to all other treatments $(\mathrm{p}<.05)$. 


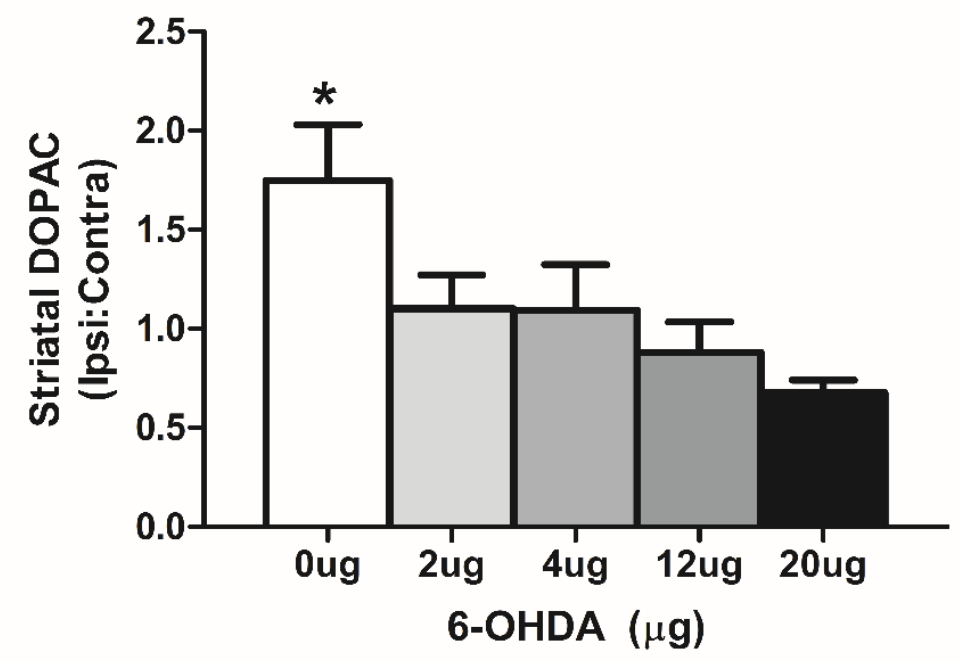

Figure 51 Appendix 1c - HPLC Striatal DOPAC

Striatal DOPAC levels at day 30 was measured using HPLC. A one way ANOVA found a significant treatment effect $(\mathrm{F}(4,33)=4.028, \mathrm{p}=$ .0091). Follow up comparisons found a significant difference between the $0 \mu \mathrm{g}$ group and all other treatment groups $(\mathrm{p}<.05)$. 


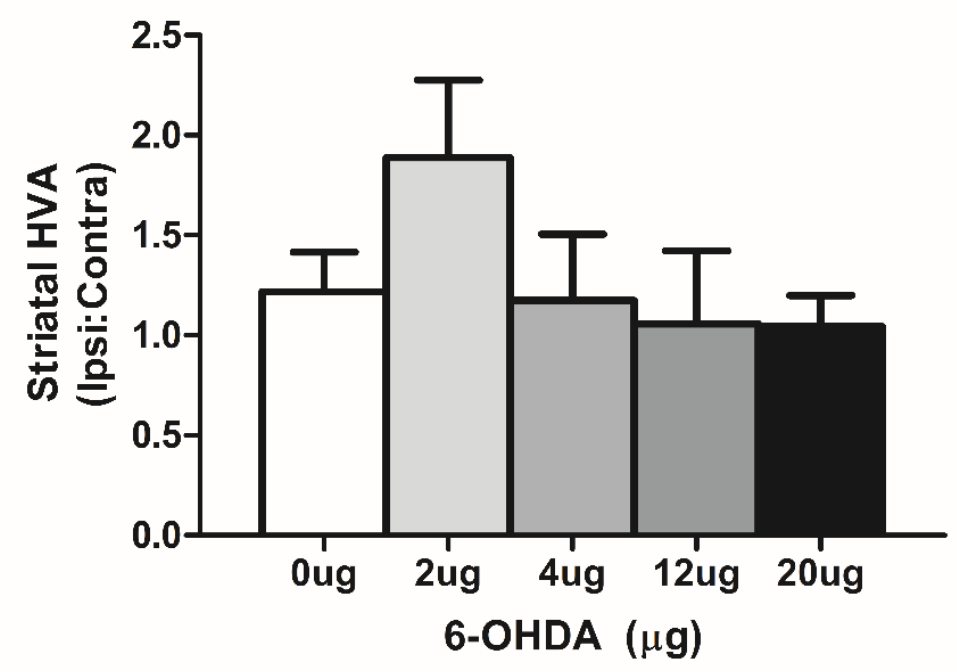

Figure 52 Appendix 1c - HPLC Striatal HVA

Striatal HVA levels at day 30 was measured using HPLC. A one way ANOVA found no significant treatment effect $(\mathrm{F}(4,33)=1.348, \mathrm{p}=$ .2731). 

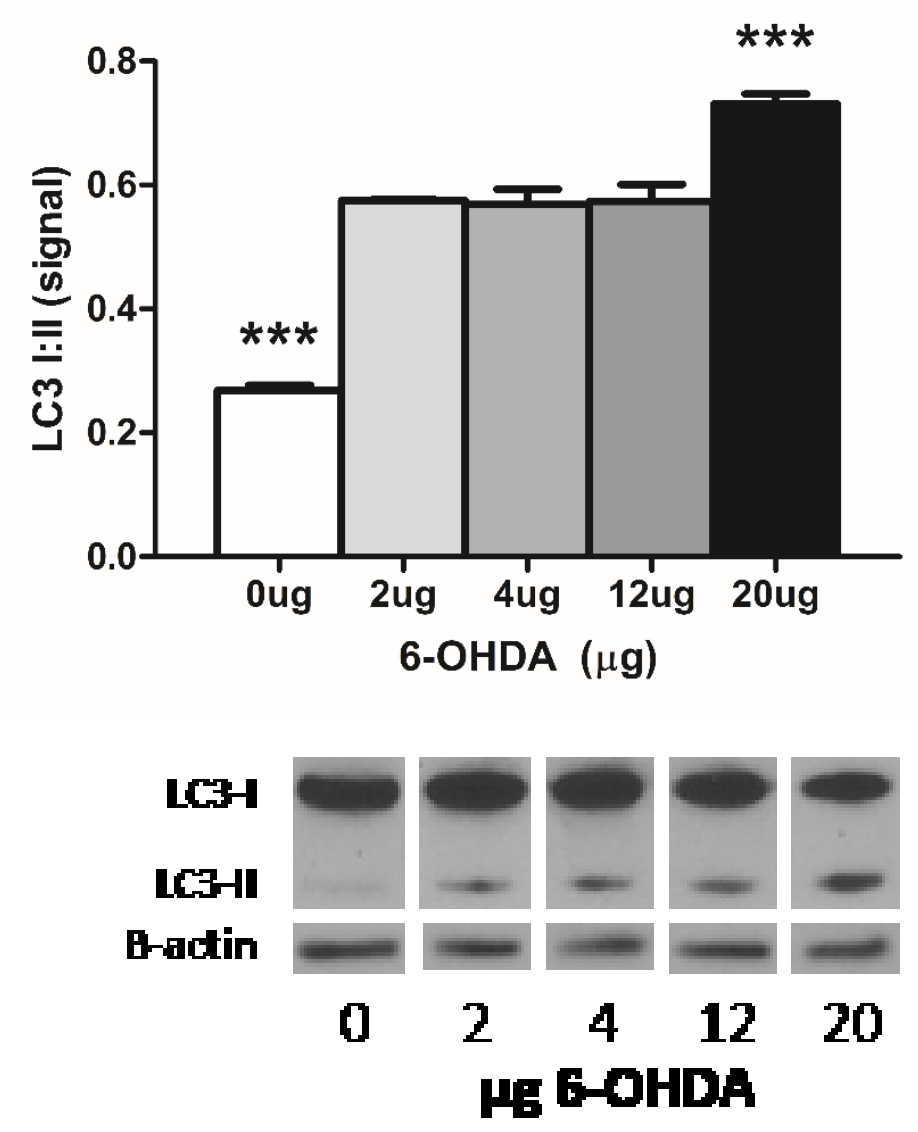

Figure 53 Appendix 1c-LC3 I:II in the striatum

LC3 I:II, a marker of autophagy, was measured in the striatum 30 days after 6-OHDA administration. A one way ANOVA found a significant treatment effect $(\mathrm{F}(4,6)=105.237, \mathrm{p}<.0001)$. Post hoc analysis found that LC3 I:II levels were significantly lower in the $0 \mu \mathrm{g}$ group. Conversely, the $20 \mu \mathrm{g}$ had significantly more LC3 I:II levels compared to all other treatments $(\mathrm{p}<.05)$. 


\section{Discussion}

The intention of this study was to develop an early stage/prodromal model of PD. We also wished to investigate further the "sick" looking cells observed in Appendix 1b, by first labeling the cells with a retrograde tracer. From this data a $20 \mu \mathrm{g}$ dose of 6-OHDA results in mild PD phenotype; minor motor deficits (Figure 47, Figure 48, Figure 49), and minimal nigrostriatal damage (Figure 50Figure 51, Figure 52). However, as we demonstrated in the earlier Model Determination Chapter we know that $20 \mu \mathrm{g}$ 6-OHDA results in a large striatal lesion and extensive substantia nigral cell loss. Exactly why in this case there was such minimal cell loss is unclear. From out post mortem location checks it appeared like the infusions were on target. It is possible that perhaps the first infusion of fluorogold caused some terminal damage, thereby reducing the uptake efficiency on the dopamine neurons and subsequently lowering the ability of 6-OHDA to induce dopaminergic neuron cell death. However, fluorogold is a commonly used retrograde tracer - if that were the case such a phenomenon would be identified in the literature, which it is not. Perhaps the canula created a micro lesion/glial scar after implantation 
damaging nerve terminals. Similarly, it could be possible that the volume injected $(2 \mu 1)$ was too large and the majority of the toxin was removed from the infusion side up the guide cannula by capillary action. Regardless of the reason, this information collected in this experiment is not usable as we have since demonstrated on many different occasions that $20 \mu \mathrm{g}$ of $6-\mathrm{OHDA}$ indeed does not produce a prodromal like state. Furthermore, our research interests evolved, and we became more interested in a clinically relevant model to test our therapeutics. 


\section{Additional Tests}

\section{Odor test}

The loss of olfaction - hyposmia - is being recognized as one of the first symptoms of PD (Factor and Weiner, 2008). Indeed hyposmia is a common symptom associated with other neurodegenerative diseases, including Alzheimer's and dementia with Lewy bodies (Factor and Weiner, 2008).

We tested two methods described in the literature to determine if we could induce an aversive response to a strong odor using unaltered/intact mice (ie. the animals had no surgery). The first method reported by Katzav (2014) involved the use of wintergreen oil - a chemical similar to menthol, however with a much stronger odor. We infused $1 \mathrm{ml}$ of solution into a 1 inch gauze pad with increasing concentrations of the wintergreen oil and recorded the amount of time each animal spent near the odor source. We found that even at $0.01 \%$ wintergreen oil the majority of the animals completely avoided the infused pad (Figure 54). For reference the concentration of wintergreen oil in many common chewing gum flavors is approximately $0.05 \%$. 
We then tested an aversion response by loosely scruffing naïve mice and placing a $1.5 \mathrm{ml}$ tube containing increasing concentrations of menthol near their nose. We expected the mice to pull their head away from the tube as a reactionary response to the strong odor. However, of the 3 mice tested not one exhibited an aversive reaction to the menthol solution, even when presented with $100 \%$ pure concentrate. Importantly we chose these test odors as other olfaction tests either rely on the use of predator odors - which would induce a sizeable cortisol stress response which we wanted to avoid - or the use of hidden foods - which require food deprivation and extensive training.

We were unable to identify a good odorant that produced a graded aversion response and had no more pilot animals to continue testing odors and methods. Unfortunately, we had to abandon this line of investigation, and no such test was implemented in our studies. 


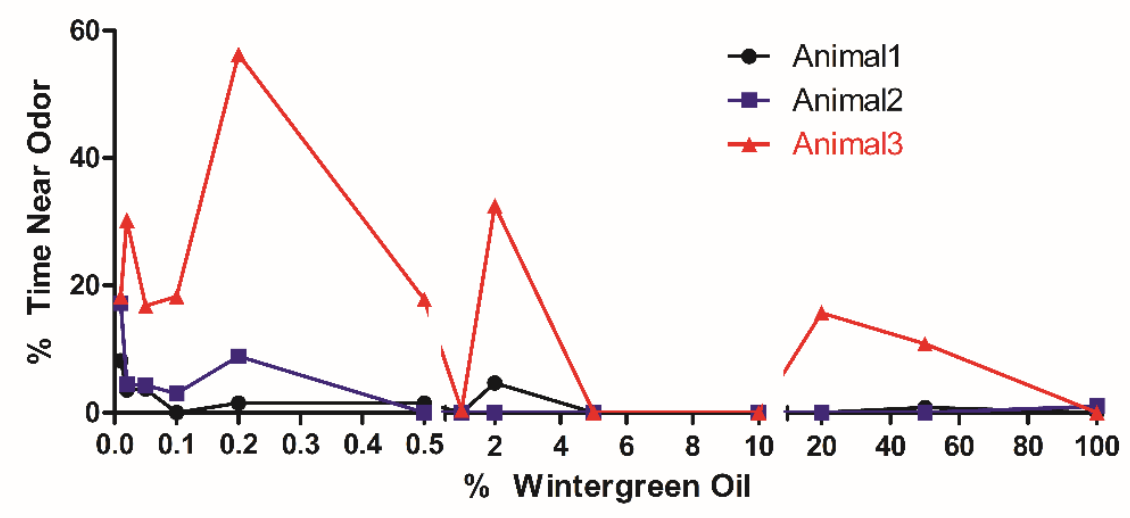

Figure 54 Appendix 1c - Wintergreen Oil Aversion Pilot

We exposed mice to varying concentrations of wintergreen oil in attempts to get a graded aversion response. Unfortunately, of the three mice tested, two of them had immediate aversion responses at $0.01 \%$, while mouse 3 demonstrated no consistent response to the odorants. 


\section{Appendix 1d}

\section{GM-CSF in a late stage model of Parkinson's disease}

\section{Introduction}

We wished to continue the line of research started in Appendix $1 \mathrm{~b}$, further investigating the potential use of cytokines as therapeutics in PD. Ashley Thompson (PhD Candidate) took over the EPO line of questioning while I continued with GM-CSF.

\section{Methods}

\section{$\underline{\text { Animals }}$}

Male CD-1 mice sourced from Charles River (Kingston, Ontario, Canada), aged 6-8 weeks upon delivery were used for all described experiments. Animals were individually housed in techniplast blue line individually ventilated polypropylene cages (approx. dimensions: 
400x200x200mm) with no enrichment other than nestlets. Animals were maintained on a standard $12 \mathrm{~h}$ light/dark cycle. Tap water and chow (Teklad, \#2014) was provided ad libitum, while room temperature and humidity were maintained at $20^{\circ} \mathrm{C}$ and $50 \%$, respectively. All aspects of this experiment were approved by the Carleton University Committee for Animal Care and adhered to the guidelines outlined by the Canadian Council for the Use and Care of Animals in Research.

\section{Surgery}

The mice were anaesthetized using variable flow isoflurane inhalational anesthetic (1.5-5\% in pure O2). Animals were then placed in a Kopf instruments Model 940 stereotaxic frame (Kopf Instruments, Tujunga, CA, USA). An L-shaped PlasticsOne 3280 P cut $3 \mathrm{~mm}$ below the pedestal was inserted into the right striatum at the coordinates $1.00 \mathrm{~mm}$ anterior, $1.75 \mathrm{~mm}$ lateral, and 3.00 ventral relative to bregma and skull surface. A single $2 \mu 1$ infusion of vehicle solution (in $0.9 \%$ saline solution containing $0.02 \%$ ascorbic acid) or 6-hydroxydopamine hydrochloride $(20 \mu \mathrm{g}$, Sigma-Aldrich, H4381) was administered at a rate of $0.4 \mu 1 /$ min using a Harvard apparatus picoplus pump equipped with a $10 \mu 1$ Hamilton syringe. The cannula was retracted after a 5 minute 
incubation period. Bonewax (Stoelting, Wood Dale, IL, USA) was placed over the drill hole and the incision closed using non-absorbable nylon sutures. Animals were given tramadol $(20 \mathrm{mg} / \mathrm{kg}$ at $2.5 \mathrm{mg} / \mathrm{ml}$, Chiron Compounding Pharmacy, Guelph, ON, Canada) twice a day for 3 days, beginning on the day of surgery. Mice were given a 30 day period before any pharmacological manipulation to ensure a maximal 6OHDA induced lesion.

\section{Pudding training}

Starting 3 weeks after the surgery, mice were introduced to our pudding administration regimen. During the 7 day training period mice were given daily access to $0.1 \mathrm{ml}$ chocolate pudding (Loblaws No Name Chocolate Pudding Cups, 20297693001_EA) on a plastic petri dish (Falcon, 35x10mm) starting immediately when the lights turn on in the morning. Day 1 mice were allowed free access for 10 minutes. Days 27 the dish was removed immediately after the mouse consumed the pudding. By the end of the training regimen most mice consume all their pudding within 1 minute. 


\section{Study Design}

Animals were equally divided into one of 5 treatment groups, detailed in Table 4 below.

\section{Drug Administration}

Thirty days following the surgery mice began, mice were given either vehicle or CTEP in a pudding suspension (Axon Medchem, Axon 1972) every $48 \mathrm{hrs}$ for 12 weeks at $2 \mathrm{mg} / \mathrm{kg}$, starting immediately when the lights turn on in the morning. Mice were also given either vehicle of GM-CSF (R\&D systems, \#415-ML) in a $0.9 \%$ saline solution via intraperitoneal injection. GM-CSF was to be given at a dose of $50 \mu \mathrm{g} / \mathrm{kg}$ every 3 weeks for a 12 week period. Following some adverse reactions, the dosing schedule was changed to $50 \mu \mathrm{g} / \mathrm{kg}$ at week 1 and then 6.25 $\mu \mathrm{g} / \mathrm{kg}$ every 6 weeks afterwards.

\section{Behavioural testing}

Prior to each behavioural test, mice were habituated to the testing room for at least a 20 minute period. 


\section{Apomorphine Induced rotations}

Animals were administered apomorphine hydrochloride hemihydrate (sub cutaneous injection, $0.5 \mathrm{mg} / \mathrm{kg}$ at $0.1 \mathrm{mg} / \mathrm{ml}$ in $0.9 \%$ saline, SigmaAldrich, A4393) 30 days following the surgical procedure to confirm parkinsonian phenotype. Any mice that received 6-OHDA and did not demonstrate net contralateral rotations were removed from the study. Briefly, immediately following the injection of apomorphine animals were placed into large open container $(27 \times 48 \times 20 \mathrm{~cm})$ and recorded from above. After a 5 minute drug incubation period, net contralateral rotations were counted over a 20 minute period. For the acute ( 1 week) study mice were challenged with apomorphine on 3 occasions -30 days after surgery, 4 hours following the bolus IP injection of CTEP, and after 1 week of CTEP administration. For the chronic (12 week study) mice were challenged with apomorphine on 2 occasions - 30 days after surgery, and again after the 12 weeks of CTEP administration. For the washout study, mice were challenged on 2 occasion - 30 days after surgery, and again after the drug washout period. 


\section{Accelerating Rota Rod}

Animals were trained on the rota rod (Omnitech Electronics) over a 2 day period. On day one animals were placed on the rota rod apparatus which was maintained at a continual speed of 11RPM. If the animal fell from the apparatus they were immediately returned over a 5 minute period. This process was repeated three times total with an inter-trial interval of 60 minutes. Day two was a repeat of Day 1, however the rota rod was maintained at 22RPM. On the third day animals were tested using an accelerating protocol with a rotation speed increasing from 4RPM to 88 RPM over a 600 second period. Maximum speed achieved before falling off the apparatus was recorded. Animals were tested over 3 trials. The lowest of the 3 trials was discarded and the average of the remaining 2 trials were used as the animals scored. The test day coincided with the last day of the experiment. To reduce test-retest confounds, animals were only tested on the rota rod on the last day of experimentation.

\section{Modified Reversal Morris Watermaze}

This protocol has been adapted from previously used methods by Nunez (Nunez, 2008) and Salmaso (Salmaso et al., 2012). Only mice in the 
chronic administration study were tested in the watermaze. Briefly, our watermaze was located in a dimly lit room. On each wall were unique identifiers for orientation. Mice received 2 days of training, the first of which occurred 2 weeks prior to experiment end, and the second of which occurred 4 days prior to experiment end. Mice were introduced into the pool from the same entry point for each trial. The platform ( $8.25 \mathrm{~cm}$ in diameter submerged $\sim 0.5 \mathrm{~cm}$ under the water surface) remained in a fixed location during the training period. Mice received two sets of 6 training trials, separated by a $1 \mathrm{hr}$ break and an inter-trial interval of 10minutes. The trial ended either when the mouse found the platform or 60 seconds had elapsed. Mice that did not find the platform were directed to platform before being removed from the pool. Immediately following the 12 th trial on training day 2 , mice were tested in the probe trial, where the platform was moved to the opposite quadrant. Time to find the platform was recorded using behavioural tracking software (ANY-maze, Stoelting). This paradigm is a measure of cognitive flexibility. 


\section{Euthanization}

Animals were randomly assigned into one of two groups - histology or western blot. Animals that were assigned to the histology group were deeply anesthetized with an overdose of sodium pentobarbital. Mice were then transcardially perfused using chilled $0.9 \%$ saline followed by 4\% PFA solution in $0.1 \mathrm{M}$ phosphate buffer. Extracted brains were post fixed for $24 \mathrm{hrs}$ in $4 \%$ PFA, followed by sequential washes of $10 \%$ sucrose in $0.1 \mathrm{M}$ phosphate buffer over another $24 \mathrm{hr}$ period. Tissue was then incubated in a $30 \%$ sucrose solution in $0.1 \mathrm{M}$ phosphate buffer for 72hrs. Following this last sucrose wash tissue was flash frozen and stored at $-80^{\circ} \mathrm{C}$. Animals that were assigned to the western blot group were euthanized by rapid decapitation. Extracted brain tissues were immediately micro dissected, placed on dry ice, and stored at $-80^{\circ} \mathrm{C}$.

\section{$\underline{\text { Statistics }}$}

One-way, Two-way, or Repeated-measurement ANOVAs were completed as appropriate. Post hoc testing was completed using Fisher's PLSD. 


\section{Table 4 Appendix 1d-GM-CSF in a late stage model of PD: Study Design}

\begin{tabular}{|c|c|c|c|c|}
\hline & Vehicle & GM-CSF & CTEP & $\begin{array}{l}\text { GM-CSF } \\
+ \text { CTEP }\end{array}$ \\
\hline 6-OHDA & & & & \\
\hline Sham & & & & \\
\hline
\end{tabular}




\section{Results}

Thirty days following the infusion of 6-OHDA animals started receiving either vehicle, CTEP, or GM-CSF over a 12 week drug administration period. Prior to the drug administration phase, all animals were challenged using apomorphine. A one-way ANOVA found a significant treatment effect $(\mathrm{F}(4,45)=7.008, \mathrm{p}=.0002)$ when comparing net contralateral rotations (Figure 55). Post hoc analysis found a significant difference between the Sham treatment group and all other groups $(\mathrm{p}<.05)$. Animals were challenged once again with apomorphine following the 12 week drug administration period. A oneway ANOVA found a significant treatment effect $(F(4,45)=16.693, p$ $<.0001$ ) (Figure 56). Post hoc analysis found that CTEP significantly reduced apomorphine induced rotations, even when given in combination with GM-CSF $(\mathrm{p}<.05)$. However, GM-CSF alone in 6OHDA treated animals was no different to saline controls $(\mathrm{p}<.05)$. When tested on the accelerating rota rod, a one way ANOVA found a significant treatment effect $(\mathrm{F}(4,45)=7.008, \mathrm{p}=.0002)($ Figure 57) Post hoc analysis found no difference between CTEP treated animals and sham controls $(\mathrm{p}<.05)$. Saline, GM-CSF, and GM-CSF+CTEP 
animals all had significantly reduced Rota Rod performance measures compared to sham controls and CTEP treated animals $(\mathrm{p}<.05)$. Animals were then measured in the modified reverse Morris water maze to assess cognitive flexibility. A one way ANOVA found a significant treatment effect $(F(4,45)=5.482, p=.0011)$ when comparing time to reach the platform (Figure 58). Post hoc analysis found that CTEP is able to reverse any 6-OHDA induced deficits compared to sham controls, even if given in combination with GM-CSF ( $p<.05)$. Comparatively, GMCSF treatment alone was not able to reverse 6-OHDA induced deficits compared to saline and sham controls $(\mathrm{p}<.05)$. 


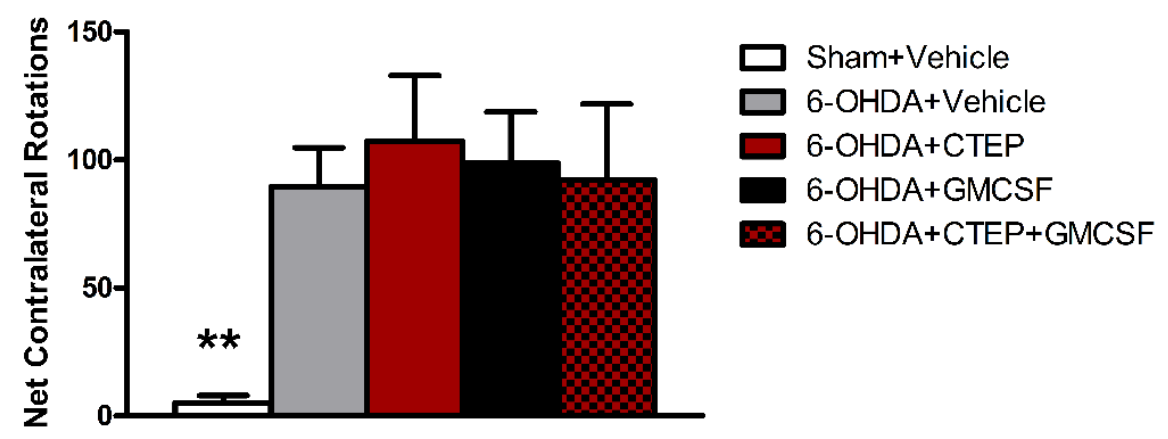

Figure 55 Appendix 1d - Apomorphine Rotations Pre-Treatment

Animals were given injections of apomorphine 30 days after administration. The net contra-lateral rotations over a 20 minute period were recorded. A one-way ANOVA found a significant treatment effect $(\mathrm{F}(4,45)=7.008, \mathrm{p}=.0002)$. Post hoc analysis found a significant difference between the Sham treatment group and all other groups $(\mathrm{p}<$ $.05)$. 


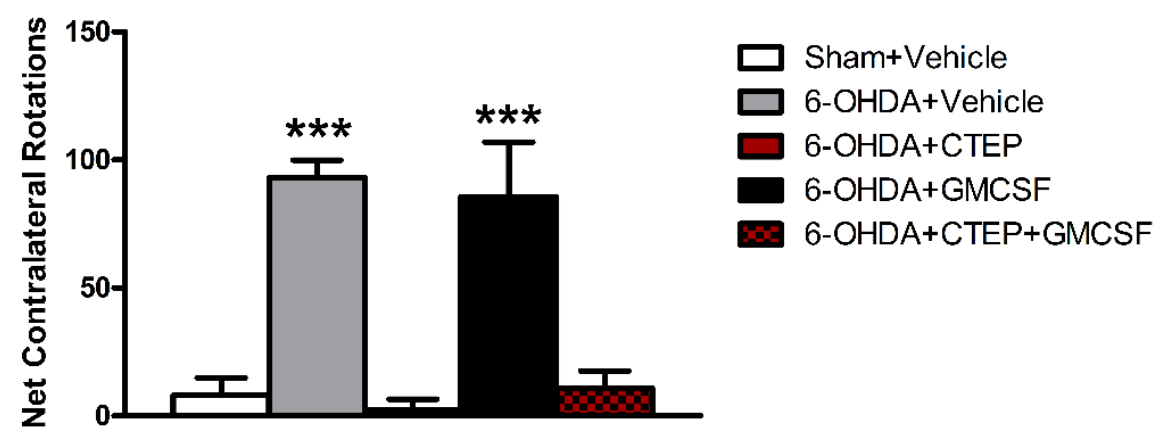

Figure 56 Appendix 1d - Apomorphine Rotations Post Treatment

Animals were given injections of apomorphine 30 days after administration. The net contra-lateral rotations over a 20 minute period were recorded. A one-way ANOVA found a significant treatment effect $(\mathrm{F}(4,45)=16.693, \mathrm{p}<.0001)$. Post hoc analysis found that CTEP significantly reduced apomorphine induced rotation, even when given in combination with GM-CSF $(p<.05)$. However GM-CSF alone was no different to saline controls $(\mathrm{p}<.05)$. 


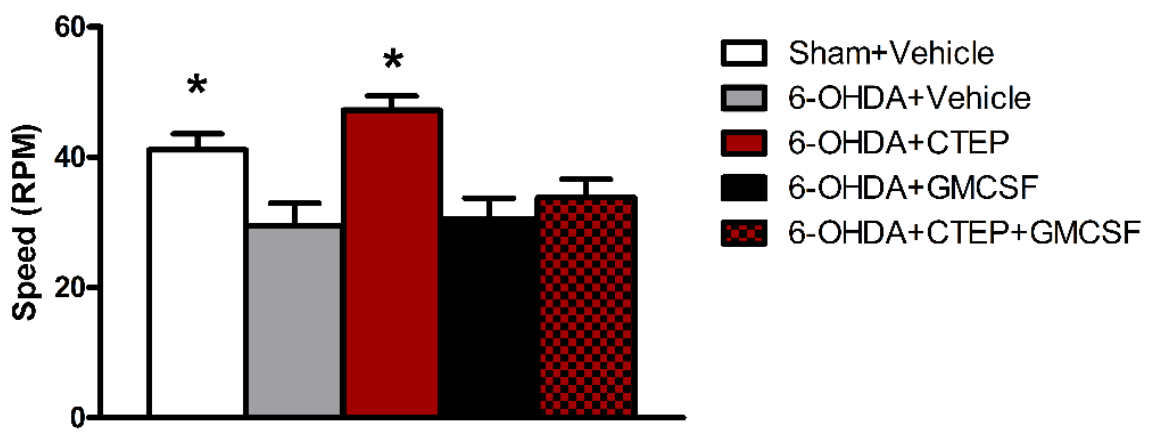

Figure 57 Appendix 1d - Rota Rod

Animals were tested on the accelerating Rota Rod after 12 weeks of drug administration. A one way ANOVA found a significant treatment effect $(\mathrm{F}(4,45)=7.008, \mathrm{p}=.0002)$. Post hoc analysis found no difference between CTEP treated animals and sham controls $(\mathrm{p}<.05)$. Saline, GMCSF, and GM-CSF+CTEP animals all had significantly reduced Rota Rod performance measures compared to sham controls $(\mathrm{p}<.05)$. 


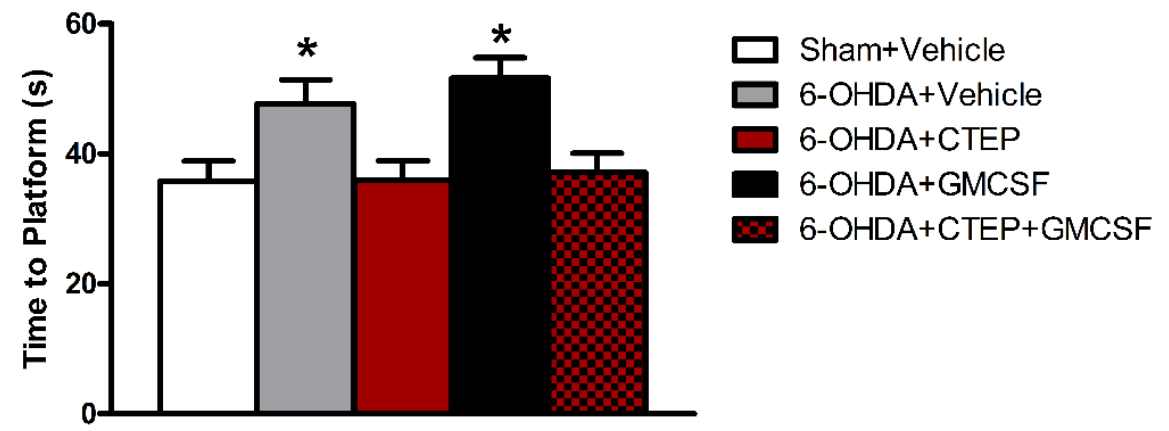

Figure 58 Appendix 1d - Watermaze

At the end of the drug administration period, animals were tested for cognitive flexibility on the reversal Morris watermaze. A one way ANOVA found a significant treatment effect $(\mathrm{F}(4,45)=5.482, \mathrm{p}=$ .0011). Post hoc analysis found that CTEP is able to reverse any 6OHDA induced deficits compared to sham controls, even if given in combination with GM-CSF $(\mathrm{p}<.05)$ comparatively GM-CSF treatment was not able to reverse 6-OHDA induced deficits compared to saline and sham controls $(\mathrm{p}<.05)$. 


\section{Discussion}

Prior to the end of the study, a change in dosing for GM-CSF was required. The dosing schedule was originally scheduled to have $50 \mu \mathrm{g} / \mathrm{kg}$ administered every 3 weeks. This dose had been used previously in the lab, including in earlier work (Appendix 1b). One common symptom associated with GM-CSF injections is general lethargy. Indeed, experimental logs indicate that quite a few of the animals experienced lethargy after the first GM-CSF treatment. Three weeks later a second injection of GM-CSF was given at $50 \mu \mathrm{g} / \mathrm{kg}$. Unexpectedly the animals died soon afterwards. The deaths occurred very quickly, taking only approximately $5-10$ mins between injection and death. The GM-CSF treated animals became quickly lethargic, followed by labored and irregular breathing. Animals also became extremely cold to the touch and their exposed skin turned blue. Postmortem dissection found there was significant abdominal edema. Interestingly animals that were given both GM-CSF and CTEP did experience similar symptoms, however survived the second injection. In total $4 / 4$ mice did not survive the second $50 \mu \mathrm{g} / \mathrm{kg}$ GM-CSF injection. As the animals were run in cohorts, a decision was made to change the 
GM-CSF dosing for the remaining animals. A final dose of $6.25 \mu \mathrm{g} / \mathrm{kg}$ was used and the injections were given less frequently. With these alterations there were no longer any lethal side effects of the cytokine therapy.

It is unclear why the animals died. Based on the rapidity and the observable symptoms prior to their death we hypothesize that the animals were experiencing some form of anaphylactic reaction to the GM-CSF injection. Indeed, the symptoms observed prior to the animals death are commonly seen in allergic reactions. Assuming that indeed what occurred was indeed an allergic reaction to GM-CSF, exactly why the animals were reacted is not clear. Indeed this dosing has been used by our lab (Chris Rudyk, Unpublished) and others (Kosloski et al., 2013) without complications. This suggests that it is not solely the toxicity of GM-CSF that is the problem, rather an interaction with the environmental conditions. Perhaps the inflammatory response to the high dose of 6-OHDA was exacerbated by the GM-CSF injections. Interestingly, when given in combination with CTEP no lethal effects were seen. This suggests that there was nothing inherently wrong with 
the GM-CSF solution itself (ie. no toxic contamination). There is evidence indicating that modulation of mGluR5 with the NAM MPEP has been shown to be effective in models of inflammatory pain (Micheli, 2000), adding further support to the anaphylactic hypothesis. After lowering our dose, we found that GM-CSF is ineffective at treating larger levels of 6-OHDA induced degeneration.

Together the data presented in Appendix 1 underscores the importance in appropriate timing of therapeutics, and heeding caution and consideration to disease state. 


\section{Appendix 2}

\section{Preface}

In addition to therapeutics we were interested in investigating potential biomarkers, something which currently does not exist in PD. Lipids, specifically lipid profiles, have recently gained traction as potential indicators of various diseases. We wanted to investigate if lipids held any potential as biomarkers of PD. To start we wished to simply determine if there were marked differences within the lipid profile within the substantia nigra. Our initial findings have been published and can be found below in Appendix 2a. Although promising, we were restricted in the types of lipids we could measure due to the type of technology available to us. As such we were limited in our ability to continue this line of questioning, and therefore focused our attentions more on therapeutic research. 


\section{Appendix 2}

\section{Major alterations of}

phosphatidylcholine and lysophosphotidylcholine lipids in the substantia nigra using an early stage model of Parkinson's disease

K. Farmer, C.A. Smith, S.P. Hayley, \& J.C. Smith

Presented as published.

International Journal of Molecular Science, 2015

DOI: $10.3390 / \mathrm{ijms} 160818865$

PMID: 26274953

\section{Abstract}

Parkinson's disease (PD) is a progressive neurodegenerative disease affecting the nigrostriatal pathway, where patients do not manifest motor symptoms until $>50 \%$ of neurons are lost. Thus, it is of great 
importance to determine early neuronal changes that may contribute to disease progression. Recent attention has focused on lipids and their role in pro- and anti-apoptotic processes. However, information regarding the lipid alterations in animal models of PD is lacking. In this study, we utilized high performance liquid chromatography electrospray ionization tandem mass spectrometry (HPLC-ESI-MS/MS) and novel HPLC solvent methodology to profile phosphatidylcholines and sphingolipids within the substantia nigra. The ipsilateral substantia nigra pars compacta was collected from rats 21 days after an infusion of 6hydroxydopamine (6-OHDA), or vehicle into the anterior dorsal striatum. We identified 115 lipid species from their mass/charge ratio using the LMAPS Lipid MS Predict Database. Of these, 19 lipid species (from phosphatidylcholine and lysophosphotidylcholine lipid classes) were significantly altered by 6-OHDA, with most being downregulated. The two lipid species that were up-regulated were LPC(16:0) and LPC(18:1), which are important for neuroinflammatory signalling. These findings provide a first step in the characterization of lipid changes in early stages of PD-like pathology and could provide novel targets for early interventions in PD. 


\section{Introduction}

Parkinson's disease (PD) is a progressive neurodegenerative disease which affects the dopaminergic nigrostriatal pathway, a brain region critical in the initiation and control of motor behaviour. The degeneration, which occurs over many decades (Bernheimer et al., 1973) eventually causes severe motor and cognitive deficits (Marié et al., 1999; Klockgether, 2004; Menza et al., 2006). The primary symptoms of PD are muscle tremors and rigidity within the distal limbs, bradykinesia (slowed movements), and general gait disturbances. Typically PD patients do not begin to manifest primary symptoms until $50-80 \%$ of the nigrostriatum has degenerated (Bernheimer et al., 1973), resulting in delayed treatments and an overall poorer prognosis. Therefore, identifying early neuronal changes which characterize the early phase of PD (early stage of disease that precedes the manifestation of full blown primary symptoms) is of great importance. Indeed, identifying biomarkers that could help classify the trajectory of illness 
in PD would greatly aid in the treatment process, as well as provide potential novel targets for the development of future therapeutics.

We have recently utilized a low dose of the specific neurotoxin 6-hydroxydopamine (6-OHDA) to induce a prodromal-like PD state. Specifically, an infusion of 6-OHDA into the dorsal striatum resulted in a modest partial lesion which encompassed $10 \%$ and $15 \%$ of the striatal volume at two and four weeks after toxin administration, respectively (Farmer et al., 2015). We also found that our model resulted in no difference in the number of neurons within the substantia nigra $(\mathrm{SNc})$; however, as would be expected in the early stages of PD, many of the neurons had morphology typically seen in unhealthy cells, specifically increased vacuolization, decreased dendritic projections, and an overall decreased cell volume (Farmer et al., 2015). Additionally, numerous other reports have indicated that pro-death oxidative and mitochondrial stress pathways are likely involved in the early stages of $\mathrm{PD}$, and that pro-inflammatory factors also arise to contribute to primary or secondary pathological processes active in the disease (Perier et al., 2007; Winklhofer and Haass, 2010; Zheng et al., 2010) . At the same time, certain genetic mutations (e.g. LRRK2, Parkin, PINK1) can lead 
to a familial form of PD or increase the vulnerability to a host of environmental insults, such as pesticides and heavy metals (Hagenah et al., 2007; Schweitzer et al., 2007). Surprisingly however, scant evidence exists regarding the potential importance of lipid alterations in PD. This is particularly surprising given that many of the toxicants implicated in PD are highly lipid soluble and in fact, accumulate to a substantial degree in brain lipid membranes (Prasad et al., 2007).

Recent attention has focused on lipids and their role in both cellsignalling pathways that govern survival or neurodegeneration. Lysophosphotidylcholines (lysoPC), for example, have been associated with pro-apoptotic processes by activating various deleterious signalling cascades, such as activation of the Bid $\mathrm{BH} 3$ protein and caspase- 3 (Goonesinghe et al., 2005). LysoPC species have also been shown to cause decreased expression of the anti-apoptotic TNF receptorassociated factor (TRAF) 2 (Qin et al., 2009). Comparatively, sphingolipids (SP) have an interesting dichotomous effect, such that they can act as both a pro-apoptotic and anti-apoptotic second messenger, wherein the difference between the two signalling pathways appears to be dependent on the site of lipid cleavage (Giussani et al., 226 
2014). Both SP and lysoPC lipid species are derived from phosphatidylcholines (PC), a lipid class which is an important mediator in various structural and signalling roles, having previously been implicated in numerous processes, such as cellular growth and survival (Wright et al., 2004). Yet, in many cases it is still unclear as to whether the effects are due to direct signalling by the lipids themselves as second messengers, or due to hyper- or hypoactivity of their associated enzymes (Wright et al., 2004; Giussani et al., 2014).

In the present investigation, we analyzed the lipid profiles evident in the substantia nigra region of rats subjected to our 6-OHDA early stage model of PD. We then compared the relative quantity and structure of PC, lysoPC and SP using HPLC-ESI-MS/MS. Importantly, we employed a novel HPLC solvent methodology to separate and identify the different lipids according to their individual mass to charge $(\mathrm{m} / \mathrm{z})$ ratio. It is our belief that this broad lipidomics profile can be used as a starting point to investigate the role of lipidomic signaling in the etiology and progression of $\mathrm{PD}$, as well as to further expand research directed at identifying detectable biomarkers of the neurodegenerative condition. 


\section{Materials and Methods}

Animals

Six male Sprague Dawley rats (Charles River), weighing between 250-280g on arrival were used in the current experiments. The animals were individually housed in a standard polypropylene cage (27 x 48 x $20 \mathrm{~cm}$ ) and maintained a 12-hour light/dark cycle. Tap water and food (Harlan Rat Chow, New Jersey) was provided ad libitum, while room temperature and humidity were maintained at $20^{\circ} \mathrm{C}$ and $50 \%$, respectively. All aspects of this experiment were approved by the Carleton University Committee for Animal Care and adhered to the guidelines outlined by the Canadian Council for the Use and Care of Animals in Research.

\section{Surgery}

The animals were anaesthetized using variable flow isoflurane inhalational anesthetic (isoflurane volume of $2-3 \%$ in pure $\mathrm{O} 2$ ). Animals 
were then placed in a Kopf instruments Model 940 stereotaxic frame with the incisor bar positioned $3.3 \mathrm{~mm}$ below the incisor line. An Lshaped PlasticsOne 3280P Osmotic Pump Cannula was implanted in the right striatum at the coordinates $1.0 \mathrm{~mm}$ anterior, $3.0 \mathrm{~mm}$ lateral and $5.0 \mathrm{~mm}$ ventral relative to bregma and skull surface. A single infusion of $20 \mu \mathrm{g}$ of 6-hydroxydopamine (Sigma, St. Louis; purchased as a hydrochloride salt, $\mathrm{n}=3$ ) in $0.9 \%$ injectable saline (containing $0.02 \%$ ascorbic acid), or vehicle solution $(\mathrm{n}=3)$, was given at a rate of 1 $\mu \mathrm{L} / \mathrm{min}$ using a Hamilton $25 \mu \mathrm{L}$ syringe with a 22 gauge needle attached to a Harvard Apparatus PicoPlus 11 Pump. The vehicle solution was made fresh daily and the 6-hydroxydopamine hydrochloride salt was mixed immediately before the infusion. After the infusion the cannula remained in place for an additional 5 minutes before being removed to ensure maximal diffusion of the 6-hydroxydopamine solution. BoneWax ${ }^{\circledR}$ was placed over the drill hole in the skull and the incision site was clipped using EZclip ${ }^{\circledR}$ surgical staples. A single application of 2\% lidocaine hydrochloride topical anaesthetic jelly (Xylocaine, AstraZeneca) was applied to the incision site. 


\section{Euthanasia}

Animals were deeply anesthetized with an injection of sodium pentobarbital and subsequently euthanized via transcardial perfusion using a chilled $0.9 \%$ saline solution 21 days after the surgical infusion. The ipsilateral substantia nigra $(\mathrm{SNc})$ was isolated from the extracted brain by dissecting the appropriate coronal section using a Kopf Instruments rat blocker obtaining a $2 \mathrm{~mm}$ slice approximately $-4.3 \mathrm{~mm}$ to $-6.3 \mathrm{~mm}$ with respect to bregma using the Paxinos \& Watson rat atlas (Paxinos and Watson, 1997). The coronal slice was then further dissected manually using a clean razor blade so as to isolate only the region of tissue containing the substantia nigra. The extracted tissue was immediately flash frozen using $100 \%$ ethanol on dry ice. All samples were stored at $-80^{\circ} \mathrm{C}$ until ready for lipid extraction.

\section{Lipid Extraction}

Animals were deeply anesthetized with an injection of sodium pentobarbital and subsequently euthanized via transcardial perfusion using a chilled $0.9 \%$ saline solution 21 days after the surgical infusion. 
The ipsilateral substantia nigra $(\mathrm{SNc})$ was isolated from the extracted brain by dissecting the appropriate coronal section using a Kopf Instruments rat blocker obtaining a $2 \mathrm{~mm}$ slice approximately $-4.3 \mathrm{~mm}$ to $-6.3 \mathrm{~mm}$ with respect to bregma using the Paxinos \& Watson rat atlas [56]. The coronal slice was then further dissected manually using a clean razor blade so as to isolate only the region of tissue containing the substantia nigra. The extracted tissue was immediately flash frozen using $100 \%$ ethanol on dry ice. All samples were stored at $-80^{\circ} \mathrm{C}$ until ready for lipid extraction.

\section{HPLC-ESI-MS/MS}

$15 \mathrm{~cm}$ lengths of $200 \mu \mathrm{m}$ inner diameter fused silica (PolymicroTechnologies, AZ, USA) were dipped in a 3:1 solution of Kasil 1678 potassium silicate and formamide (Promega, WI, USA) to make a column frit on one end. The columns were allowed to dry for 24 hours, were shortened to $10 \mathrm{~cm}$, and subsequently packed with $5 \mathrm{~cm}$ of $5 \mu \mathrm{m}$ ReproSil-Pur C4 stationary phase beads (Dr. Maisch GmbH, Germany) in acetone using a nitrogen pressure vessel. A PicoFrit 
Emitter (New Objective MA, USA) was filled in a similar manner, however only $1 \mathrm{~cm}$ of ReproSil-Pur C4 beads were used.

Test samples were prepared immediately before each run $(1 \mu \mathrm{L}$ of concentrated lipid sample, $5 \mu \mathrm{L}$ of absolute ethanol, and $34 \mu \mathrm{L}$ of deionized water). Samples were placed into a Dionex UltiMate 3000 autosampler (Thermo Fisher Scientific, Waltham, MA) maintained at 4 ${ }^{\circ} \mathrm{C}$ and loaded onto the chromatography column using a Dionex UltiMate 3000 pump (Thermo Fisher Scientific, Waltham, MA, $20 \mu \mathrm{L}$ total volume). All mobile phases were prepared using HPLC grade solvents: (A) $30 \%$ methanol in $10 \mathrm{mM}$ ammonium acetate, (B) isopropanol with $10 \mathrm{mM}$ ammonium acetate and (C) hexane. Each lipid mass analysis was 60 minutes in length and began with a $100 \%$ A mobile phase. Four minutes later solvent B was gradually increased until the mobile phase became $100 \% \mathrm{~B}$ at the 45.5 minute mark where is remained at $100 \% \mathrm{~B}$ for 1 minute. At the 46.6 minute mark solvent $\mathrm{C}$ gradually increased until the mobile phase became $100 \% \mathrm{C}$ where it remained until the end of the run. 
Chromatographically separated lipids were directly analyzed using an AB Sciex 4000 QTRAP ESI-MS/MS Hybrid Triple Quadrupole/Linear Ion Trap (AB Sciex, Framingham, MA). The QTRAP was run in positive ion mode, with the ESI nanospray voltage set at $3 \mathrm{kV}$, curtain gas at 20 and declustering potential at $25 \mathrm{~V}$. A precursor ion scan and an enhanced mass spectrum (EMS) were conducted using a mass range of 250-1500 Da. The precursor ion scan was set to analyze precursors of $\mathrm{m} / \mathrm{z} 184$ with a collision energy of 40.0 $\mathrm{eV}$ and collision cell exit potential of $10 \mathrm{~V}$.

Immediately after each lipid sample was analyzed, a 140 minute hexane wash was run $(90 \% \mathrm{C}$ and $10 \% \mathrm{~B})$. Towards the end of the wash at the 130 minute mark the mobile phase switched to $100 \% \mathrm{~B}$ and before switching to $100 \% \mathrm{~A}$ at 137 minutes. A 40 minute blank was run immediately following the wash cycle to ensure there was no carry over. The mobile phase began with $100 \%$ A before gradually switching to $100 \% \mathrm{~B}$ at starting a 4 minutes and ending at 28 minutes. The mobile phase remained at $100 \% \mathrm{~B}$ for another minute before returning to $100 \%$ A until the end of the run. All samples were run in triplicate. 


\section{Data Anylsis}

Mass spectra were analyzed using Analyst Software version 1.5.1 (AB Sciex). The precursor m/z 184 scan was manually analyzed to generate a list of phosphatidylcholine and sphingolipid m/z-values. The m/z-values (+/-0.2Da) were then entered into MultiQuant 2.1.1 (AB Sciex). All desired peaks were integrated and an output containing a variety of parameters, such as peak area and retention time, was generated. The peak area of each lipid was divided by the peak area of the internal standard C13:0 LPC (m/z 454), and then again by the total weight of the substantia nigra. The triplicate data was averaged for each animal providing a biological $\mathrm{n}$ of 3 per treatment group. LIPID MAPS MS Prediction Tool (Lipidomics Gateway) was then used to determine the classification and to predict the structure of all lipid masses identified in the precursor $\mathrm{m} / \mathrm{z} 184$ scan. Statistical significance between treatment groups for each lipid species was determined using independent-samples t-tests. 


\section{Results}

Our lipidomics profile scan identified 115 different lipid species, of these, $19(16.5 \%)$ were significantly changed in the 6-OHDA treated animals. We found that 17 (89.5\%) of these significantly altered lipids were down-regulated and only $2(10.5 \%)$ were up-regulated. PC species were primarily affected accounting for $63.2 \%$ of all significantly altered lipids (12/19), and were found to be entirely down-regulated (Figure 59). Similarly, we found that lysoPC species were mainly downregulated (Figure 60); however two notable lysoPC lipids were upregulated; $\mathrm{LPC}(16: 0)$ and $\mathrm{LPC}(18: 1)$. The two upregulated lysoPC lipids were also found to be in the highest relative concentrations, with normalized peak area values in the 6-OHDA treated animals being 379 and 294 for LPC(16:0) and (18:1) respectively. Comparatively, the average normalized peak area for all other lysoPC lipids is 1.84 , and 1.90 for PC lipids. Finally, we found that $36.8 \%$ of the significantly altered lipids contained ether-linked acyl chains. A summary of our lipidomic detection findings can be found in Table 5 . 


\section{Table 5 Appendix 2 - Summary table of lipidomic}

findings.

Summary table of lipidomic findings. The class of lipids was determined using the LIPID MAPS MS Prediction Tool.

\begin{tabular}{|c|c|}
\hline & $\mathrm{n}=$ \\
\hline Lipids Identified & 115 \\
\hline Lipids with Significant Changes & 19 \\
\hline Lipids up-regulated in 6-OHDA Animals & 2 \\
\hline Lipids down-regulated in 6-OHDA Animals & 17 \\
\hline Phosphatidylcholines with Significant Changes & 12 \\
\hline Lysophosphatidylcholines with Significant Changes & 7 \\
\hline
\end{tabular}




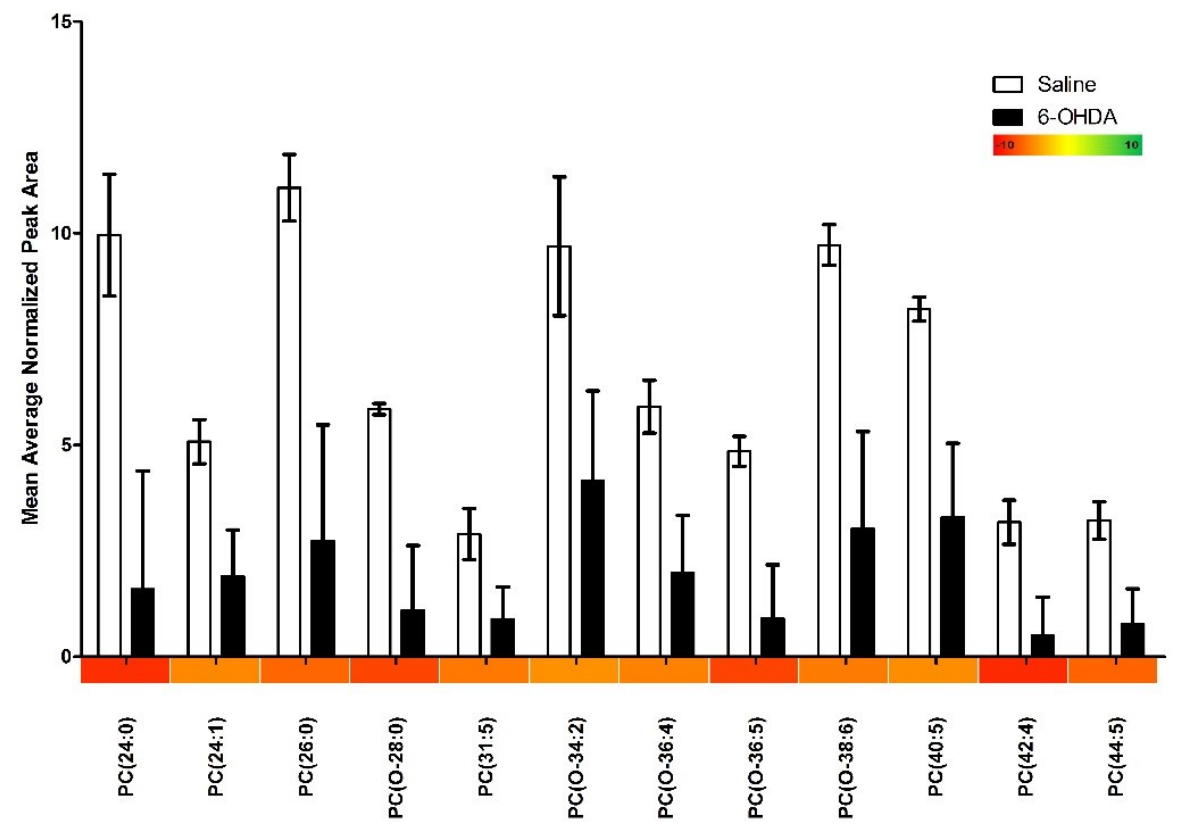

Figure 59 Appendix 2 - Changes in Phosphatidylcholines

The relative abundance of phosphatidylcholine species in the substantia nigra of animals treated with either 6-OHDA $(20 \mu \mathrm{g})$ or saline vehicle into the right anterior dorsal striatum, as determined by HPLC-ESI$\mathrm{MS} / \mathrm{MS}$ measurements. All measurements were run in triplicate and the error bars represent the standard error. Only species displaying significant differences between treatment and control groups are shown. Significance was determined by independent sample t-test. The relative fold change of phosphatidylcholine species is represented along the $\mathrm{X}$ axis in the form of a color heat map. 


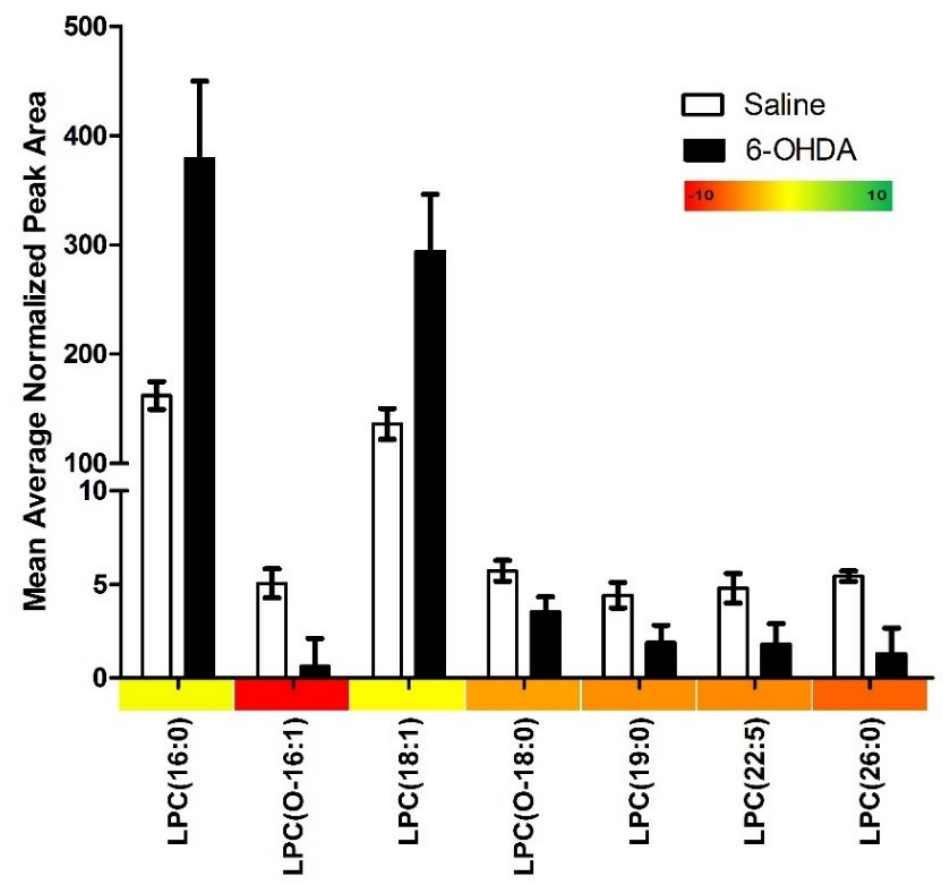

Figure 60 Appendix 2 - Changes in lysophosphatidylcholines

The relative abundance of phosphatidylcholine species in the substantia nigra of animals treated with either 6-OHDA $(20 \mu \mathrm{g})$ or saline vehicle into the right anterior dorsal striatum, as determined by HPLC-ESI$\mathrm{MS} / \mathrm{MS}$ measurements. All measurements were run in triplicate and the error bars represent the standard error. Only species displaying significant differences between treatment and control groups are shown. Significance was determined by independent sample t-test. The relative fold change of phosphatidylcholine species is represented along the $\mathrm{X}$ axis in the form of a color heat map. 


\section{Discussion}

In this study we used a relatively low dose of 6-OHDA to induce a partial nigrostriatal lesion, which we believe may partially model a prodromal-like PD state. We have recently found that this animal model causes modest striatal lesions, coupled with morphological abnormalities of surviving neurons (Farmer et al., 2015). In the present study, we found that PC lipid species were markedly altered in animals treated with 6-OHDA. By far, PC species were the most affected and were generally down-regulated in all cases. Importantly, PC lipids are major components of many cell structures (Wright et al., 2004), including being present with exceptionally high levels in vacuole membranes (Gulshan and Moye-Rowley, 2011). The possibility exists that the decrease of PC lipids in 6-OHDA treated mice reflects some degree of structural rearrangement. In effect, as neurons develop PD like pathology (e.g. decreased dendritic projections, decreased cell volume and cell death) the cell may simply require significantly lower PC lipid concentrations. 
In addition to structural support, PC lipids may also contribute to functional aspects of neuronal processes by influencing various signalling pathways. For instance, previous studies have reported that PC lipids modulate anti-inflammatory signaling (Treede et al., 2007). Moreover, it is known that 6-OHDA can induce microglial reactivity (Marinova-Mutafchieva et al., 2009; Stott and Barker, 2013; Farmer et al., 2015) and elevations of pro-inflammatory cytokines (Choudhury et al., 2011; Goes et al., 2013; Hernandes et al., 2013b). Thus, the decrease in PC lipids currently observed may influence cell survival in the context of 6-OHAD treatment by influencing inflammatory processes.

Although 6-OHDA can provoke pro-apoptotic effects through the protein kinase $\mathrm{C} \delta(\mathrm{PKC} \delta$ ) complex system (Hanrott et al., 2006), this same system can also impart anti-apoptotic consequences through diacylglycerol (DAG) molecules, which are primary PC lipid metabolites (Wright and McMaster, 2002; Argaud et al., 2004). This raises the possibility that the array of lipids modulated by 6-OHDA are likely engaged in a sort of cellular "tug of war" concerning neuronal survival pathways. Another potential source of anti-apoptotic DAG is via sphingomyelin synthase, an enzyme that combines a ceramide and 240 
PC lipid to cause the synthesis of a SP and DAG molecule (Wright et al., 2004). Although our findings did not show any significant changes in SP lipids, we did find trends in which up-regulations of sphingosine and sphinganine approached significance.

Similar to our PC results, we found an overall decrease in lysoPC species among 6-OHDA treated animals. However, there were two notable exceptions, such that LPC (16:0) and (18:1) were greatly increased in 6-OHDA treated animals. LysoPC are lipids synthesized from PCs via the enzyme phospholipase A2 (PLA2). 6-OHDA has been reported to induce PLA2 enzymatic activity within the nigrostriatal tract (Hayakawa et al., 1998; Lee et al., 2010). Interestingly, increased PLA2 activity has been repeatedly associated with apoptotic effects (Jaattela et al., 1995; Kim et al., 2002) including 6-OHDA induced apoptosis (Ahmad et al., 2013). However the role of PLA2 is exceedingly complex, as the enzyme has also been implicated in anti-apoptotic pathways, primarily via the arachidonic acid and peroxisome proliferator-activated receptor (PPAR) signalling pathway (Kreisler et al., 2007). Arachidonic acid and PPAR system activation has also been shown to reduce oxidative stress and normalize mitochondrial 
dysfunction (Kreisler et al., 2007; Ravnsjkjaer et al., 2014); both of which are affected by 6-OHDA (Khan et al., 2010; Asaithambi et al., 2014; Kupsch et al., 2014; Park et al., 2014). Interestingly, both LPC (16:0) and LPC(18:1) have been shown to play major roles in regulating PLA2 enzyme activity by acting as an uncompetitive inhibitor (Cunningham et al., 2008). Alternatively, the accumulation of these LPC species could be related to impairment of other enzymes in the PC re-acylation or de-acylation processes. However, as the literature is lacking on the role of these other enzymes in PD and PD-like pathology, with further enzymology follow up studies required.

It is also possible that the observed increase in LysoPC species (16:0) and (18:1) is unrelated to altered 6-OHDA induced PLA2 enzymatic activity, and instead could be increased to serve a very specific and targeted role by acting as immune signalling mediators. In this regard, it has been shown that LysoPC (16:0) and (18:1) play a major role in inflammatory signaling (Cunningham et al., 2008), including the release of various cytokines (interlukin-1 $\beta$, interlukin-6, chemokine ligand 2 , chemokine ligand 4 , and tumor necrosis factor alpha) (Liu-Wu et al., 1998; Gonçalves et al., 2012). Importantly, the 242 
two LPC species have also have potent chemotactic abilities, being able to induce and recruit macrophages and T-lymphocytes to injured tissue (Quinn et al., 1988; McMurray et al., 1993; Ryborg et al., 1994; Liu-Wu et al., 1998). Given these findings, it is possible that the overall decrease of lysoPC species, as a class of lipids, is related to changes in PLA2 activity, whereas, the dramatic increase in LysoPC (16:0) and (18:1) may arise from their involvement in inflammatory processes induced by 6-OHDA. Finally, it is important to point out that we currently report relative differences between the various species rather than absolute lipid levels. Indeed, we are most interested in lipid profile changes that might be characteristic of early stages PD.

In addition to "typical" $\mathrm{PC}$ and lysoPC species, we identified numerous "atypical" ether linked forms of these species; all of which were down-regulated. In agreement with our current findings, it has also been reported that PD patients had significantly decreased levels of ether-linked lipids (Fabelo et al., 2011). Ether linked lipids are known to generally be platelet activating factors, and have been shown to be heavily involved in the neuroinflammatory responses (Braverman and Moser, 2012). Importantly, drugs that antagonize ether-linked 243 
phospholipids have potential clinical relevance given their reported neuroprotective and anti-tumorigenic effects (Lohmeyer and Bittman, 1994; Dave et al., 2005).

It is of considerable importance to underscore that we currently assessed lipid levels in the SNc, which normally sends projections "downstream" to the striatum to modulate basal ganglia activity. Thus, the observed lipid changes are presumably upstream from the neurotoxin-injected striatum, indicating that they likely stem from retrograde signalling pathways. Indeed, the view is widely held that PD begins with a loss of function and degeneration of the axon terminals in the striatum, which then progresses back to the cell bodies in the SNc (Galvin et al., 1999; van der Putten et al., 2000; Lotharius and Brundin, 2002; Cheng et al., 2010). Further to this point, the intra-striatal 6OHDA model of PD is also known to induce progressive neuronal degeneration over numerous weeks (Sauer and Oertel, 1994; Blandini et al., 2008) and causes increased neuroinflammatory responses (Choudhury et al., 2011; Goes et al., 2013; Hernandes et al., 2013a). Additionally, we used a low dose of 6-OHDA which we have recently reported to cause a modest but significant loss of dopamine (DA) striatal 
terminals, but does not significantly affect the number of neurons within SNc (Farmer et al., 2015). Taken together, these findings suggest that the intra-striatal 6-OHDA model of PD can be an important tool for the investigation of the early stages of $\mathrm{PD}$ as it mimics the human condition in many key aspects.

Mitochondrial dysfunction is an important contributor to PD pathology, and indeed 6-OHDA acts primarily via disruption of mitochondrial processes, resulting in the generation of reactive oxygen species (Kupsch et al., 2014; Segura-Aguilar and Kostrzewa, 2015). It is important to note, however, that our assay results in the extraction of general cellular and not specific mitochondrial lipids per se. Methods to isolate mitochondrial lipids in a manner that renders them amenable to MS analysis are difficult to perform and introduce a great deal of variability.

In the current study, we can only identify the general structure of the various lipid species. Identifying the full fatty acid composition can be achieved with further MS2 analysis using either lithium or acetate adduction methods. However, these methods pose significant 
limitations, as they are effective for standards, but can be problematic when using complex tissue samples (as they become difficult to separate chromatographically). Both adduction methods are also substantially less sensitive than those involving the production of protonated phosphocholine fragment ions, such as the precursor m/z 184 scan in positive ion mode (as outlined in the detailed methods section) was used in the current study.

Besides identifying novel lipids that could be mechanistically involved in $\mathrm{PD}$, the present findings could conceivably have implications for the development of biomarkers. Of course, for a molecule to be a useful biomarker it must be readily detectable in peripheral tissue and/or fluids. In this regard, a recent study detected increases in 9 ceramide class lipids in the plasma of PD patients (Mielke et al., 2013). Moreover, several PC and lysoPC lipid alterations were found in the plasma of patients with Alzheimer's disease (Mapstone et al., 2014). Future studies are required to assess whether 6-OHDA induces peripheral lipid changes that parallel the presently observed brain lipid changes. However, current evidence is promising and the use 
of lipidomics screens as a potential tool for early diagnosis of PD may play an important role in the future.

\section{Conclusions}

We report that 21 days after an intrastriatal infusion of a low dose of 6-OHDA there are significant decreases in PC and lysoPC within the SNc. Two lipids, LysoPC (16:0) and (18:1) stood out in that they greatly upregulated by 6-OHDA. Although speculative, these observed lipid changes may contribute to structural reconfiguration, immune system signalling, and pro- and anti-apoptotic pathways. This broad lipidomic profile is novel and might be used to further investigate the role of specific lipid species in the etiology and progression of PD, as well as providing potential biomarkers for PD progression. Of course, to be ultimately useful in this capacity, future work will be required to determine whether peripheral lipid species change in parallel with the presently observed SNc variations. Finally, it is particularly noteworthy that the series of marked lipid changes occurred within the SNc, which is of course, up-stream of the 6-OHDA injected striatum. Thus, our findings clearly indicate that retrograde signals likely emanated from the 
partially lesioned striatum to modulate lipid composition within the SNc cell soma. 


\section{Appendix 3 Spontaneous re-innervation in the 6-OHDA model of Parkinson's disease}

\section{Introduction}

As the title suggests, this appendix section is dedicated to discussing spontaneous re-innervation in the 6-OHDA model of Parkinson's disease. Herein I discuss a large problem inherent in many murine models. Indeed there is evidence of spontaneous recovery in various models of PD including MPTP (Poli et al., 1992; Mitsumoto et al., 1998) and 6-OHDA (Dravid et al., 1984; Blanchard et al., 2002; Stanic et al., 2003). PD models are not the only ones affected. Recently it was found that a popular triple mutant model of Alzheimer's disease is also affected by spontaneous recovery (Donating Investigator Report to Jackson Labs, Strain: 004807). The original intent of this study was to complete a long term washout following 12 weeks of CTEP 
administration. This experiment was to assess whether the striatal changes imparted by CTEP after extended administration resulted in sustained recovery, or behavioural regression as was described earlier following 1 week of CTEP treatment. Unfortunately, the length of time between administration of 6-OHDA and experimental endpoint was too long, as will become apparent in the following sections.

\title{
Methods
}

\author{
$\underline{\text { Animals }}$ \\ Male CD-1 mice sourced from Charles River (Kingston, Ontario, \\ Canada), aged 6-8 weeks upon delivery were used for all described \\ experiments. Animals were individually housed in techniplast blue line \\ individually ventilated polypropylene cages (approx. dimensions: \\ 400x200x200mm) with no enrichment other than nestlets. Animals were \\ maintained on a standard $12 \mathrm{~h}$ light/dark cycle. Tap water and chow \\ (Teklad, \#2014) was provided ad libitum, while room temperature and \\ humidity were maintained at $20^{\circ} \mathrm{C}$ and $50 \%$, respectively. All aspects of \\ this experiment were approved by the Carleton University Committee
}


for Animal Care and adhered to the guidelines outlined by the Canadian Council for the Use and Care of Animals in Research.

\section{$\underline{\text { Surgery }}$}

The mice were anaesthetized using variable flow isoflurane inhalational anesthetic $(1.5-5 \%$ in pure O2). Animals were then placed in a Kopf instruments Model 940 stereotaxic frame (Kopf Instruments, Tujunga, CA, USA). An L-shaped PlasticsOne $328 \mathrm{OP}$ cut $3 \mathrm{~mm}$ below the pedestal was inserted into the right striatum at the coordinates $1.00 \mathrm{~mm}$ anterior, $1.75 \mathrm{~mm}$ lateral, and 3.00 ventral relative to bregma and skull surface. A single $2 \mu 1$ infusion of vehicle solution (in $0.9 \%$ saline solution containing $0.02 \%$ ascorbic acid) or 6-hydroxydopamine

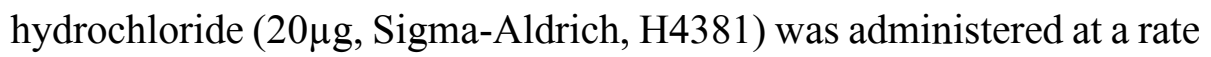
of $0.4 \mu 1 /$ min using a Harvard apparatus picoplus pump equipped with a $10 \mu 1$ Hamilton syringe. The cannula was retracted after a 5 minute incubation period. Bonewax (Stoelting, Wood Dale, IL, USA) was placed over the drill hole and the incision closed using non-absorbable nylon sutures. Animals were given tramadol $(20 \mathrm{mg} / \mathrm{kg}$ at $2.5 \mathrm{mg} / \mathrm{ml}$, Chiron Compounding Pharmacy, Guelph, ON, Canada) twice a day for 3 days, beginning on the day of surgery. Mice were given a 30 day 
period before any pharmacological manipulation to ensure a maximal 6OHDA induced lesion.

\section{Pudding training}

Starting 3 weeks after the surgery, mice were introduced to our pudding administration regimen. During the 7 day training period mice were given daily access to $0.1 \mathrm{ml}$ chocolate pudding (Loblaws No Name Chocolate Pudding Cups, 20297693001_EA) on a plastic petri dish (Falcon, 35x10mm) starting immediately when the lights turn on in the morning. Day 1 mice were allowed free access for 10 minutes. Days 27 the dish was removed immediately after the mouse consumed the pudding. By the end of the training regimen most mice consume all their pudding within 1 minute.

\section{CTEP}

Thirty days following the surgery, mice were given either vehicle or CTEP (Axon Medchem, Axon 1972) every 48hrs for 12 consecutive weeks at $2 \mathrm{mg} / \mathrm{kg}$, starting immediately when the lights turn on in the morning. 


\section{Drug Washout}

Following the 12 weeks of drug administration, animals were given a 4 week drug washout prior to any behavioural testing and Euthanization.

\section{Behavioural testing}

Prior to each behavioural test, mice were habituated to the testing room for at least a 20 minute period.

\section{Apomorphine Induced rotations}

Animals were administered apomorphine hydrochloride hemihydrate (sub cutaneous injection, $0.5 \mathrm{mg} / \mathrm{kg}$ at $0.1 \mathrm{mg} / \mathrm{ml}$ in $0.9 \%$ saline, SigmaAldrich, A4393) 30 days following the surgical procedure to confirm parkinsonian phenotype. Any mice that received 6-OHDA and did not demonstrate net contralateral rotations were removed from the study. Briefly, immediately following the injection of apomorphine animals were placed into large open container $(27 \times 48 \times 20 \mathrm{~cm})$ and recorded from above. After a 5 minute drug incubation period, net contralateral rotations were counted over a 20 minute period. Animals were rechallenged with apomorphine at the end of the drug washout out period. 


\section{Accelerating Rota Rod}

Animals were trained on the rota rod (Omnitech Electronics) over a 2 day period. On day one animals were placed on the rota rod apparatus which was maintained at a continual speed of 11RPM. If the animal fell from the apparatus they were immediately returned over a 5 minute period. This process was repeated three times total with an inter-trial interval of 60 minutes. Day two was a repeat of Day 1, however the rota rod was maintained at 22RPM. On the third day animals were tested using an accelerating protocol with a rotation speed increasing from 4 RPM to 88 RPM over a 600 second period. Maximum speed achieved before falling off the apparatus was recorded. Animals were tested over 3 trials. The lowest of the 3 trials was discarded and the average of the remaining 2 trials were used as the animals scored. The test day coincided with the last day of the experiment. To reduce test-retest confounds, animals were only tested on the rota rod on the last day of experimentation. 


\section{Modified Reversal Morris Watermaze}

This protocol has been adapted from previously used methods by (Nunez, 2008) and (Salmaso et al., 2012). Briefly, our watermaze was located in a dimly lit room. On each wall were unique identifiers for orientation. Mice received 2 days of training, the first of which occurred 2 weeks prior to experiment end, and the second of which occurred 4 days prior to experiment end. Mice were introduced into the pool from the same entry point for each trial. The platform $(8.25 \mathrm{~cm}$ in diameter submerged $\sim 0.5 \mathrm{~cm}$ under the water surface) remained in a fixed location during the training period. Mice received two sets of 6 training trials, separated by a $1 \mathrm{hr}$ break and an inter-trial interval of 10 minutes. The trial ended either when the mouse found the platform or 60 seconds had elapsed. Mice that did not find the platform were directed to platform before being removed from the pool. Immediately following the 12th trial on training day 2 , mice were tested in the probe trial, where the platform was moved to the opposite quadrant. Time to find the platform was recorded using behavioural tracking software (ANY-maze, Stoelting). This test is a measure of cognitive flexibility. 


\section{Euthanization}

Animals were randomly assigned into one of two groups - histology or western blot. Animals that were assigned to the histology group were deeply anesthetized with an overdose of sodium pentobarbital. Mice were then transcardially perfused using chilled $0.9 \%$ saline followed by 4\% PFA solution in $0.1 \mathrm{M}$ phosphate buffer. Extracted brains were post fixed for $24 \mathrm{hrs}$ in $4 \%$ PFA, followed by sequential washes of $10 \%$ sucrose in $0.1 \mathrm{M}$ phosphate buffer over another $24 \mathrm{hr}$ period. Tissue was then incubated in a $30 \%$ sucrose solution in $0.1 \mathrm{M}$ phosphate buffer for 72hrs. Following this last sucrose wash tissue was flash frozen and stored at $-80^{\circ} \mathrm{C}$. Animals that were assigned to the western blot group were euthanized by rapid decapitation. Extracted brain tissues were immediately micro dissected, placed on dry ice, and stored at $-80^{\circ} \mathrm{C}$.

\section{Histology}

Tissue was sectioned at $40 \mu \mathrm{m}$ on a rotary cryostat. Free floating tissue sections were immune-labelled overnight in primary antibodies (mouse anti-TH 1:2000 [Immunostar 22941]). Tissue sections were incubated in biotinylated secondary and tertiary streptavidin HRP solutions for 2hrs each. Using ImageJ software at the site of injection and the two 
adjoining sites, integrated densities were measured as a ratio to its ipsilateral hemisphere signals after background subtraction. $\mathrm{TH}+$ cells amounts of the SNc were assessed in a qualitative manner by a blinded experimenter $(0=$ no noticeable cell loss, $1=$ minor cell loss, $2=$ approx $50 \%$ cell loss, $3=$ extensive cell loss). 


\section{Results}

Thirty days following infusion of 6-OHDA, animals were challenged with apomorphine to confirm parkinsonian phenotype. When comparing net contralateral apomorphine induced rotations a oneway ANOVA found a significant treatment effect $(F(2,27)=43.961, p$ $<$.0001) (Figure 61). Post hoc analysis found that 6-OHDA treated animals rotated significantly more compared to Sham treated animals ( $p$ $<.001)$. Animals were then treated with either CTEP or vehicle for 12 weeks followed by a 4 week drug washout period. After the end of the drug washout, animals were tested for a battery of behaviours. When comparing net contralateral apomorphine induced rotations a one-way ANOVA found no significant treatment effect $(F(2,27)=0.197, p=$ .8225) (Figure 62). When tested on the accelerating rota rod a one way ANOVA also found no significant treatment effect $(F(2,26)=1.598, p$

$=.2216)$ (Figure 63). Similarly, when tested for cognitive flexibility in a modified reversal Morris Watermaze a one way ANOVA found no significant treatment effect $(\mathrm{F}(2,27)=0.969, \mathrm{p}=.3922)($ Figure 64). 
Because of the null results in the behaviours, histology was completed on only a small subset of animals. When comparing the integrated density values of TH labelled striatal sections, a one way ANOVA found no significant treatment effect $(F(2,3)=1.762, p=$ .3119) (Figure 65). When comparing TH labelled nigral sections using a qualitative rating scale, a one-way ANOVA finds a significant difference $(\mathrm{F}(2,3)=21.000, \mathrm{p}=.0172)$ (Figure 66). Post hoc analysis found that both the vehicle and CTEP treated animals where not different from each other, however both had significantly more $\mathrm{TH}+$ cell loss in the SNc compared to sham controls $(\mathrm{p}<.05)$. 


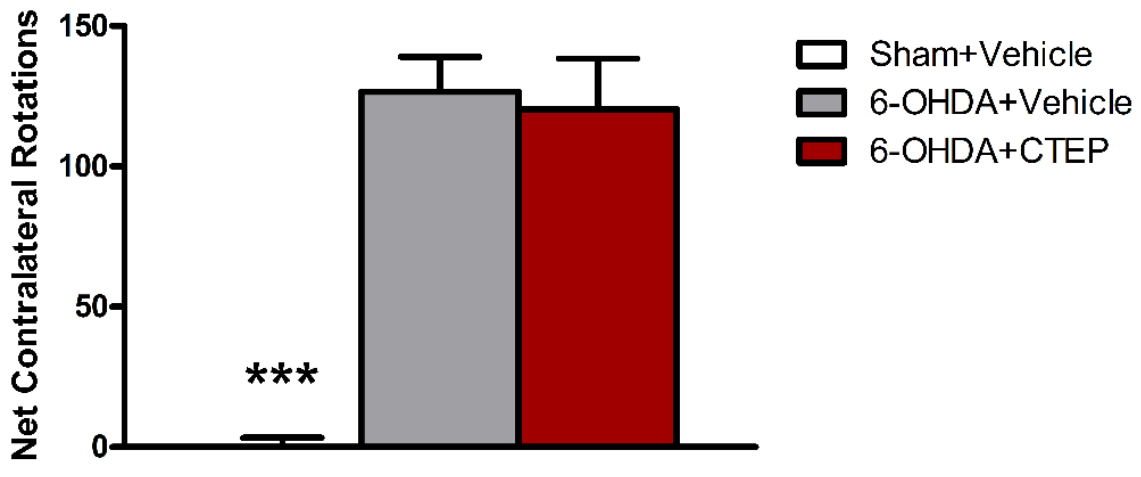

Figure 61 Appendix 3 - Apomorphine Rotations Pre Treatment

Animals were given injections of apomorphine 30 days after administration. The net contra-lateral rotations over a 20 minute period were recorded. A one-way ANOVA found a significant treatment effect $(\mathrm{F}(2,27)=43.961, \mathrm{p}<.0001)$. Post hoc analysis found a significant difference between the Sham treatment group and all other groups $(\mathrm{p}<$ $.001)$. 


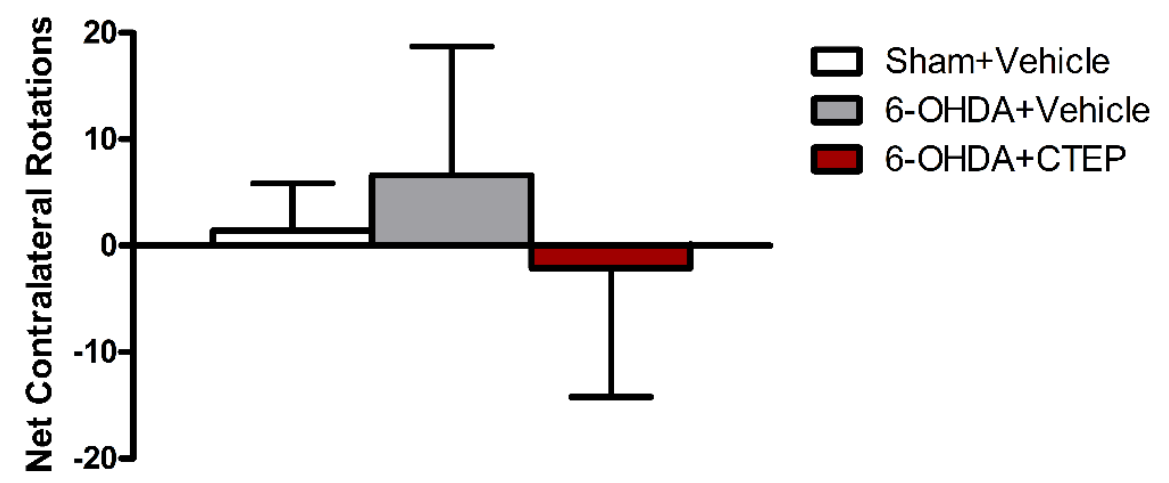

Figure 62 Appendix 3 - Apomorphine Rotations Post Washout

Animals were given injections of apomorphine after 12 weeks of drug administration and 4 weeks of drug washout. The net contra-lateral rotations over a 20 minute period were recorded. A one-way ANOVA found no significant treatment effect $(\mathrm{F}(2,27)=0.197, \mathrm{p}=.8225)$ 


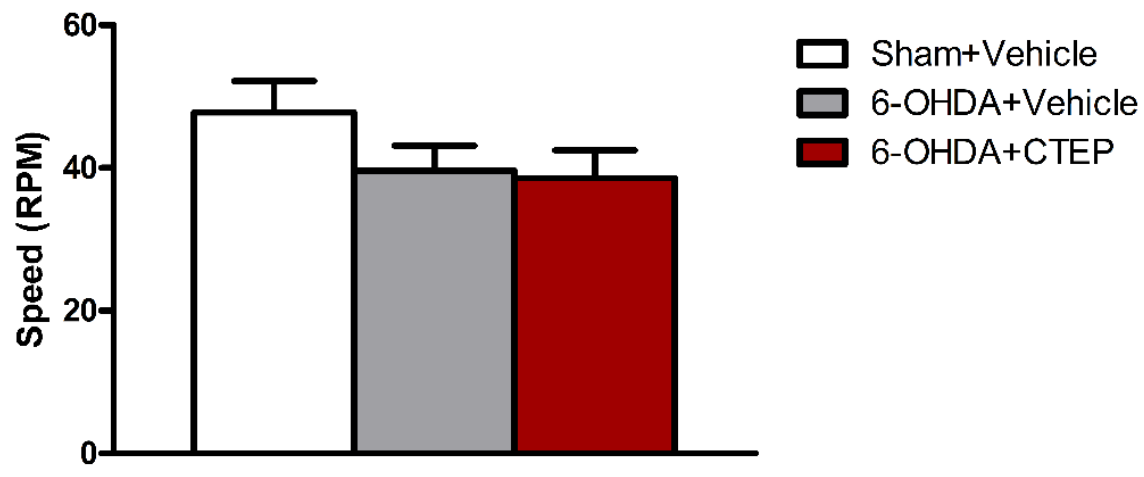

Figure 63 Appendix 3 - Rota Rod

Animals were tested on the accelerating Rota Rod after 12 weeks of drug administration and 4 weeks of drug washout. A one way ANOVA found no significant treatment effect $(\mathrm{F}(2,26)=1.598, \mathrm{p}=.2216)$. 


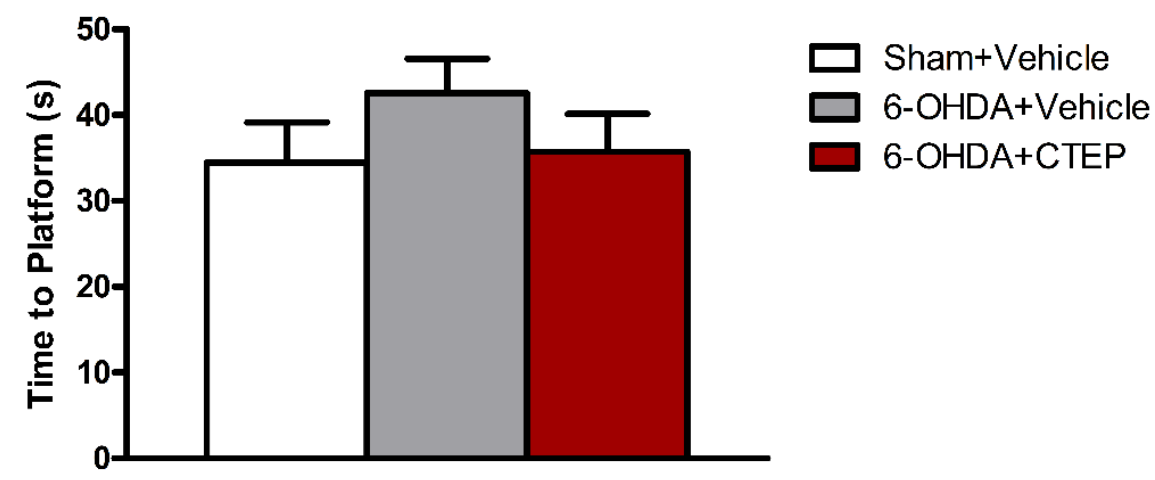

Figure 64 Appendix 3 - Watermaze

Animals were tested for cognitive flexibility on the reversal Morris watermaze after 12 weeks of drug administration and 4 weeks of drug washout. A one way ANOVA found no significant treatment effect $(\mathrm{F}(2,27)=0.969, \mathrm{p}=.3922)$. 

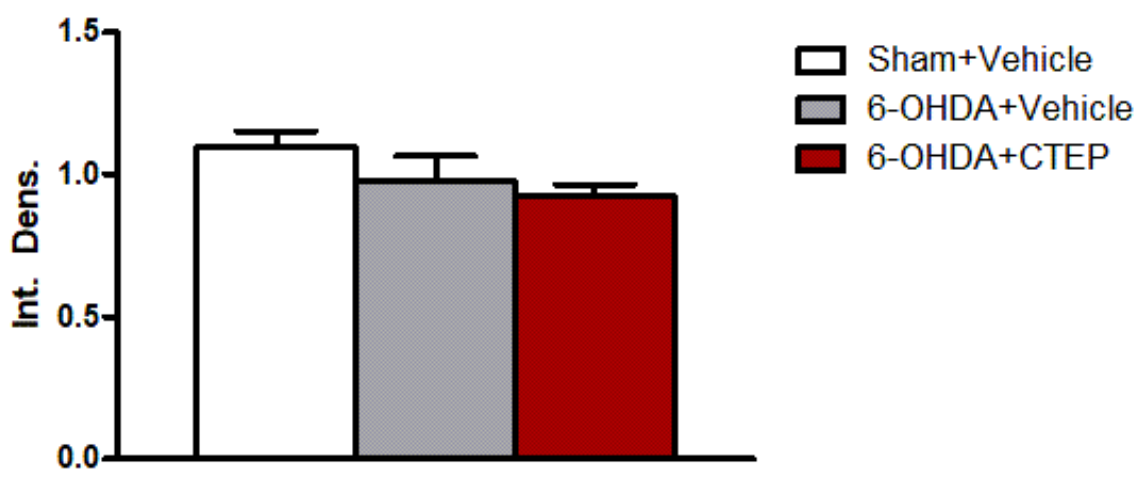

Sham+Vehlcle

6-OHDA+Vehicle

6-OHDA+CTEP
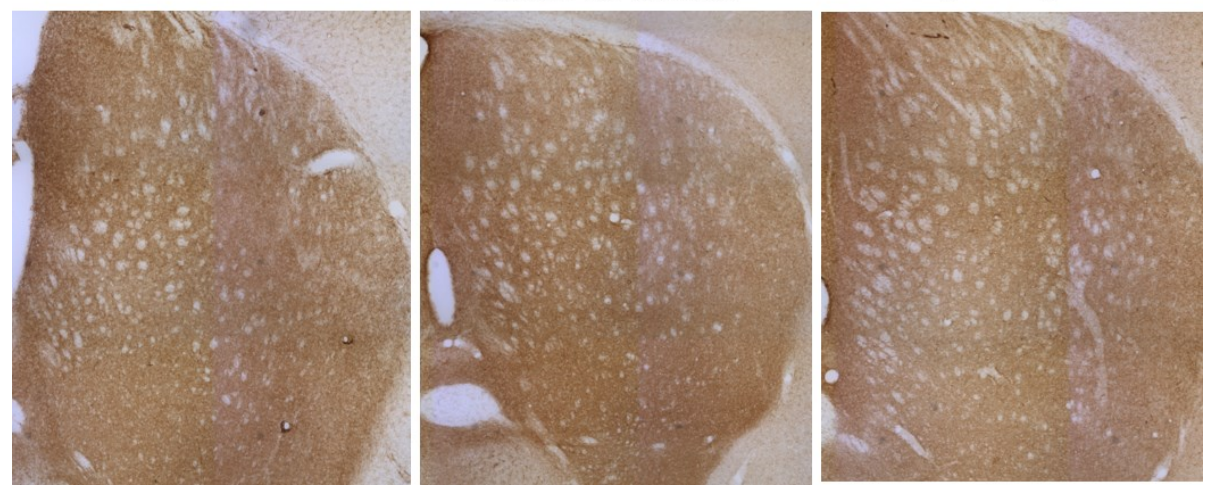

Figure 65 Appendix 3 - Striatal TH Integrated Density

The integrated density of striatal sections immunolabelled with $\mathrm{TH}$ was measured using ImageJ software for a small sample of animals. A one way ANOVA found no significant treatment effect $(\mathrm{F}(2,3)=1.762, \mathrm{p}=$ .3119). 


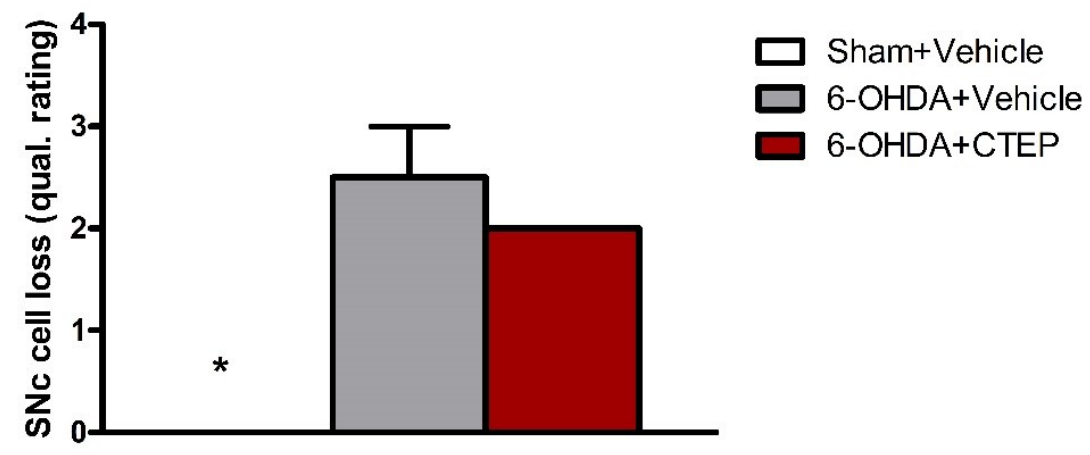

\section{Ipsilateral Contralateral}
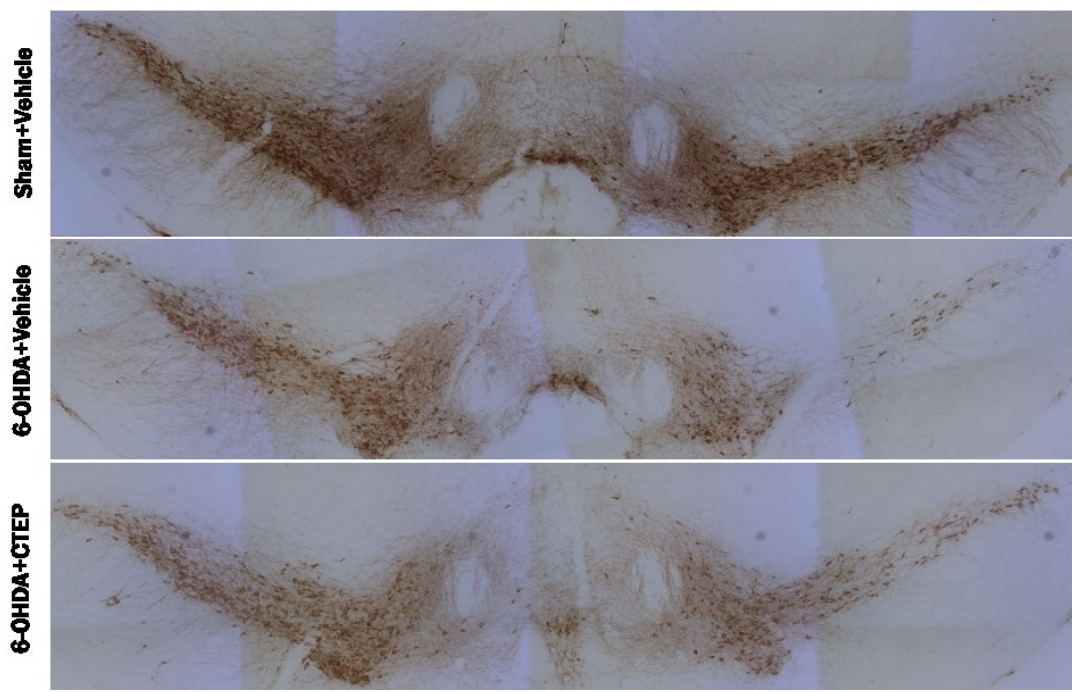

Figure 66 Appendix 3 - SNc cell loss

A TH stain was performed on serial sections of the SNc. A blind qualitative analysis of a small sample of animals comparing ipsilateral and contralateral sides was completed on the stained sections $(0=$ no noticeable cell loss, $1=$ minor cell loss, $2=$ approx. $50 \%$ cell loss, $3=$ extensive cell loss). A one way ANOVA found a significant treatment effect $(F(2,3)=21.000, p=.0172)$. A post hoc analysis found that both the vehicle and CTEP treated animals had significantly more cell loss in the SNc compared to sham controls $(\mathrm{p}<.05)$. 


\section{Discussion}

With this experiment, an unintended outcome was the recapitulation of previous findings that demonstrated spontaneous recovery following 6-OHDA treatments. While spontaneous recovery in mice is usually accompanied by a recovery of striatal dopamine, often the nigra does not recover (Dravid et al., 1984; Blanchard et al., 2002; Stanic et al., 2003). However, some report functional recovery in the absence of dorsal striatal recovery (Rothblat and Schneider, 1994), suggesting there is some form of potential downstream compensatory mechanisms. In all likelihood what is occurring is a mixture of both situations - spontaneous DA recovery with additional downstream GABAergic and glutamatergic compensation.

No model rodent toxin model is perfect or immune to such potential spontaneous recovery. Even the catastrophically quick damage induced by MPTP has been known to be affected by eventual spontaneous recovery (Poli et al., 1992; Mitsumoto et al., 1998). The 6OHDA model is excellent for modelling progressive nigrostriatal loss, capable of producing everything from a small lesion to complete 266 
ablation of the nigral DA system. Additionally, the 6-OHDA model allows for targeted administration that you can control with excellent accuracy and precision compared to other systemic models. While it appears that the inability to carry out longitudinal studies is a limitation of the 6-OHDA model, it by no means negates its overall use as a model of PD. 


\section{Figure Licences}

Figure 1 Traditional signalling cascade of the Group I mGluR receptors

License Number: 4265390437689

License Date: Jan 10, 2018

Licenced Content Publisher: Elsevier

Licensed Content Publication: Pharmacological Research

Licensed Content Title: Metabotropic glutamate receptors and neurodegenerative diseases

Licensed Content Author: Fabiola M. Ribeiro, Luciene B. Vieira, Rita G.W. Pires, Roenick P. Olmo, Stephen S.G. Ferguson

Licensed Content Date: Jan 1, 2017

Type of use: Dissertation/Thesis

Portion: Figure/Table

Original Wiley Figure/Table Number(s): Fig 1C

Requestor: Kyle Farmer

Requestor Location: Carleton University

1125 Colonel By Drive

Ottawa, ON, Canada

K1S 5B6 


\section{References}

Abd-elrahman KS, Hamilton A, Hutchinson SR, Liu F, Russell RC, Ferguson SSG (2017) mGluR5 antagonism increases autophagy and prevents disease progression in the $\mathrm{zQ} 175$ mouse model of Huntington's disease. Sci Signal 10:1-12.

Abe N, Borson SH, Gambello MJ, Wang F, Cavalli V (2010) Mammalian Target of Rapamycin (mTOR) Activation Increases Axonal Growth Capacity of Injured Peripheral Nerves. J Biol Chem 285:28034-28043 Available at: http://www.jbc.org/lookup/doi/10.1074/jbc.M110.125336.

Ahmad AS, Maruyama T, Narumiya S, Doré S (2013) PGE2 EP1 receptor deletion attenuates 6-OHDA-induced Parkinsonism in mice: old switch, new target. Neurotox Res 23:260-266.

Ai Y, Markesbery W, Zhang Z, Grondin R, Elseberry D, Gerhardt GA, Gash DM (2003) Intraputamenal infusion of GDNF in aged rhesus monkeys: Distribution and dopaminergic effects. J Comp Neurol 461:250-261.

Altar CA, Boylan CB, Jackson C, Hershenson S, Miller J, Wiegand SJ, Lindsay RM, Hyman C (1992) Brain-derived neurotrophic factor augments rotational behavior and nigrostriatal dopamine turnover 
in vivo. Proc Natl Acad Sci U S A 89:11347-11351 Available at: http://www.ncbi.nlm.nih.gov/pubmed/1454818.

Alvarez-Fischer D, Henze C, Strenzke C, Westrich J, Ferger B, Höglinger GU, Oertel WH, Hartmann A (2008) Characterization of the striatal 6-OHDA model of Parkinson's disease in wild type and alpha-synuclein-deleted mice. Exp Neurol 210:182-193.

Ambrosi G, Armentero M-TM-T, Levandis G, Bramanti P, Nappi G, Blandini F (2010) Effects of early and delayed treatment with an mGluR5 antagonist on motor impairment, nigrostriatal damage and neuroinflammation in a rodent model of Parkinson's disease. Brain Res Bull 82:29-38 Available at: http://www.ncbi.nlm.nih.gov/pubmed/20100549 [Accessed November 14, 2013].

Argaud L, Prigent A-F, Chalabreysse L, Loufouat J, Lagarde M, Ovize M (2004) Ceramide in the antiapoptotic effect of ischemic preconditioning. Am J Physiol Heart Circ Physiol 286:H246-51.

Asaithambi A, Ay M, Jin H, Gosh A, Anantharam V, Kanthasamy A, Kanthasamy AG (2014) Protein Kinase D1 (PKD1) Phosphorylation Promotes Dopaminergic Neuronal Survival during 6-OHDA-Induced Oxidative Stress. PLoS One 9:e96947 Available at:

http://www.pubmedcentral.nih.gov/articlerender.fcgi?artid=40130 $52 \&$ tool=pmcentrez\&rendertype $=$ abstract $[$ Accessed May 12, $2014]$. 
Austin P, Betts M, Broadstock M, O’Neill M, Mitchell S, Duty S (2010) Symptomatic and neuroprotective effects following activation of nigral group III metabotropic glutamate receptors in rodent models of Parkinson's disease. Br J Pharmacol 160:17411753 Available at: http://doi.wiley.com/10.1111/j.14765381.2010.00820.x.

Bagga V, Dunnett SB, Fricker RA (2015) The 6-OHDA mouse model of Parkinson's disease - Terminal striatal lesions provide a superior measure of neuronal loss and replacement than median forebrain bundle lesions. Behav Brain Res 288:107-117 Available at: http://linkinghub.elsevier.com/retrieve/pii/S0166432815002235.

Barber A, Farmer K, Martin KR, Smith PD (2015) Retinal regeneration mechanisms linked to multiple cancer molecules: A therapeutic conundrum. Prog Retin Eye Res.

Barlow B, Coryslechta D, Richfield E, Thiruchelvam M (2007) The gestational environment and Parkinson's disease: Evidence for neurodevelopmental origins of a neurodegenerative disorder. Reprod Toxicol 23:457-470 Available at: http://linkinghub.elsevier.com/retrieve/pii/S0890623807000263.

Bashkatova VG, Sudakov SK (2012) Role of Metabotropic Glutamate Receptors in the Mechanisms of Experimental Parkinsonism Development. Bull Exp Biol Med 153:655-657 Available at: http://link.springer.com/10.1007/s10517-012-1790-9. 
Batchelor PE, Liberatore GT, Wong JY, Porritt MJ, Frerichs F, Donnan G a, Howells DW (1999) Activated macrophages and microglia induce dopaminergic sprouting in the injured striatum and express brain-derived neurotrophic factor and glial cell linederived neurotrophic factor. J Neurosci 19:1708-1716.

Bennouar K-E, Uberti MA, Melon C, Bacolod MD, Jimenez HN, Cajina M, Kerkerian-Le Goff L, Doller D, Gubellini P (2013) Synergy between 1-DOPA and a novel positive allosteric modulator of metabotropic glutamate receptor 4: Implications for Parkinson's disease treatment and dyskinesia. Neuropharmacology 66:158-169 Available at: http://linkinghub.elsevier.com/retrieve/pii/S0028390812001219.

Bensadoun JC, Mirochnitchenko O, Inouye M, Aebischer P, Zurn AD (1998) Attenuation of 6-OHDA-induced neurotoxicity in glutathione peroxidase transgenic mice. Eur J Neurosci 10:32313236 Available at: http://www.ncbi.nlm.nih.gov/pubmed/9786216.

Berg D et al. (2011) AFQ056 treatment of levodopa-induced dyskinesias: results of 2 randomized controlled trials. Mov Disord 26:1243-1250 Available at: http://www.ncbi.nlm.nih.gov/pubmed/21484867.

Bernasconi E, Favre L, Maillard MH, Bachman D, Pythoud C, Bouzourene H, Croze E, Velichko S, Parkinson J, Michetti P, Velin D (2010) Granulocyte-macrophage colony-stimulating 
factor elicits bone marrow-derived cells that promote efficient colonic mucosal healing. Inflamm Bowel Dis 16:428-441.

Bernheimer H, Birkmayer W, Hornykiewicz O, Jellinger K, Seitelberger F (1973) Brain dopamine and the syndromes of Parkinson and Huntington. Clinical, morphological and neurochemical correlations. J Neurol Sci 20:415-455.

Betts MJ, O'Neill MJ, Duty S (2012) Allosteric modulation of the group III mGlu4 receptor provides functional neuroprotection in the 6-hydroxydopamine rat model of Parkinson's disease. $\mathrm{Br} \mathrm{J}$ Pharmacol 166:2317-2330 Available at: http://doi.wiley.com/10.1111/j.1476-5381.2012.01943.x.

Bezard E, Dovero S, Prunier C, Ravenscroft P, Chalon S, Guilloteau D, Crossman AR, Bioulac B, Brotchie JM, Gross CE (2001) Relationship between the appearance of symptoms and the level of nigrostriatal degeneration in a progressive 1-methyl-4-phenyl1,2,3,6-tetrahydropyridine-lesioned macaque model of Parkinson's disease. J Neurosci 21:6853-6861.

Bezard E, Pioli EY, Li Q, Girard F, Mutel V, Keywood C, Tison F, Rascol O, Poli SM (2014) The mGluR5 negative allosteric modulator dipraglurant reduces dyskinesia in the MPTP macaque model. Mov Disord 29:1074-1079.

Binder DK, Scharfman HE (2004) Brain-derived neurotrophic factor. Growth Factors 22:123-131 Available at: 
http://www.ncbi.nlm.nih.gov/pubmed/15518235.

Black YD, Xiao D, Pellegrino D, Kachroo A, Brownell A-LL, Schwarzschild MA (2010) Protective effect of metabotropic glutamate mGluR5 receptor elimination in a 6-hydroxydopamine model of Parkinson's disease. Neurosci Lett 486:161-165 Available at: http://dx.doi.org/10.1016/j.neulet.2010.09.043.

Blanchard V, Chritin M, Vyas S, Savasta M, Feuerstein C, Agid Y, Javoy-Agid F, Raisman-Vozari R (2002) Long-Term Induction of Tyrosine Hydroxylase Expression: Compensatory Response to Partial Degeneration of the Dopaminergic Nigrostriatal System in the Rat Brain. J Neurochem 64:1669-1679 Available at: http://www.ncbi.nlm.nih.gov/pubmed/7891094 [Accessed November 27, 2017].

Blandini F, Armentero MT, Martignoni E (2008) The 6hydroxydopamine model: news from the past. Parkinsonism Relat Disord 14 Suppl 2:S124-9.

Blandini F, Levandis G, Bazzini E, Nappi G, Armentero M-T (2007) Time-course of nigrostriatal damage, basal ganglia metabolic changes and behavioural alterations following intrastriatal injection of 6-hydroxydopamine in the rat: new clues from an old model. Eur J Neurosci 25:397-405.

Blandini F, Porter R, Greenamyre JT (1996) Glutamate and Parkinson's disease. Ann Neurol 12:73-95. 
Blesa J, Phani S, Jackson-Lewis V, Przedborski S (2012) Classic and New Animal Models of Parkinson's Disease. J Biomed Biotechnol 2012:1-10 Available at: http://www.ncbi.nlm.nih.gov/pubmed/845618.

Bobyn J, Mangano EN, Gandhi A, Nelson E, Moloney K, Clarke M, Hayley S (2012) Viral-toxin interactions and Parkinson's disease: poly I:C priming enhanced the neurodegenerative effects of paraquat. J Neuroinflammation 9:86 Available at: http://www.pubmedcentral.nih.gov/articlerender.fcgi?artid=34647 26\&tool=pmcentrez\&rendertype $=$ abstract $[$ Accessed June 16, $2014]$.

Bond WS, Rex TS (2014) Evidence That Erythropoietin Modulates Neuroinflammation through Differential Action on Neurons, Astrocytes, and Microglia. Front Immunol 5:31-35 Available at: http://journal.frontiersin.org/article/10.3389/fimmu.2014.00523/a bstract [Accessed December 22, 2014].

Bouhy D, Malgrange B, Multon S, Poirrier A-LL, Scholtes F, Schoenen J, Franzen R (2006) Delayed GM-CSF treatment stimulates axonal regeneration and functional recovery in paraplegic rats via an increased BDNF expression by endogenous macrophages. FASEB J 20:1239-1241 Available at: http://www.ncbi.nlm.nih.gov/pubmed/16636109 [Accessed April 29, 2013].

Bourn WM, Chin L, Picchioni AL (1972) Enhancement of audiogenic 275 
seizure by 6-hydroxydopamine. J Pharm Pharmacol 24:913-914 Available at: http://www.ncbi.nlm.nih.gov/pubmed/4405660.

Boyd TD, Bennett SP, Mori T, Governatori N, Runfeldt M, Norden M, Padmanabhan J, Neame P, Wefes I, Sanchez-Ramos J, Arendash GW, Potter H (2010) GM-CSF upregulated in rheumatoid arthritis reverses cognitive impairment and amyloidosis in Alzheimer mice. J Alzheimers Dis 21:507-518 Available at: http://www.ncbi.nlm.nih.gov/pubmed/20555144 [Accessed November 14, 2013].

Braak H, Del Tredici K, Rüb U, de Vos RAI, Jansen Steur ENH, Braak E (2003) Staging of brain pathology related to sporadic Parkinson's disease. Neurobiol Aging 24:197-211 Available at: http://www.ncbi.nlm.nih.gov/pubmed/12498954.

Braverman NE, Moser AB (2012) Functions of plasmalogen lipids in health and disease. Biochim Biophys Acta 1822:1442-1452.

Breese GR, Traylor TD (1971) Depletion of brain noradrenaline and dopamine by 6-hydroxydopamine. Br J Pharmacol 42:88-99 Available at: http://www.ncbi.nlm.nih.gov/pubmed/5580702.

Breit S, Schulz JB, Benabid A-L (2004) Deep brain stimulation. Cell Tissue Res 318:275-288 Available at: http://link.springer.com/10.1007/s00441-004-0936-0.

Brundin P, Isacson O, Gage F, Prochiantz A, Bjorklund A (1986) The rotating 6-hydroxydopamine-lesioned mouse as a model for 
assessing functional effects of neuronal grafting. Brain Res 366:346-349.

Caldwell AB, Cheng Z, Vargas JD, Birnbaum HA, Hoffmann A (2014) Network dynamics determine the autocrine and paracrine signaling functions of TNF. Genes Dev 28:2120-2133 Available at: http://genesdev.cshlp.org/lookup/doi/10.1101/gad.244749.114.

Carriere A, Romeo Y, Acosta-Jaquez HA, Moreau J, Bonneil E, Thibault P, Fingar DC, Roux PP (2011) ERK1/2 Phosphorylate Raptor to Promote Ras-dependent Activation of mTOR Complex 1 (mTORC1). J Biol Chem 286:567-577 Available at: http://www.jbc.org/lookup/doi/10.1074/jbc.M110.159046.

Carvalho AL, Caldeira M V, Santos SD, Duarte CB (2009) Role of the brain-derived neurotrophic factor at glutamatergic synapses. $\mathrm{Br} \mathrm{J}$ Pharmacol 153:S310-S324 Available at: http://doi.wiley.com/10.1038/sj.bjp.0707509.

Carvey PM, Chang Q, Lipton JW, Ling Z (2003) Prenatal exposure to the bacteriotoxin lipopolysaccharide leads to long-term losses of dopamine neurons in offspring: a potential, new model of Parkinson's disease. Front Biosci 8:s826-s837.

Chan H, Paur H, Vernon AC, Zabarsky V, Datla KP, Croucher MJ, Dexter DT (2010) Neuroprotection and Functional Recovery Associated with Decreased Microglial Activation Following Selective Activation of mGluR2/3 Receptors in a Rodent Model 
of Parkinson's Disease. Parkinsons Dis 2010:1-12 Available at: http://www.ncbi.nlm.nih.gov/pubmed/20948891.

Charvin D (2018) mGlu4allosteric modulation for treating Parkinson's disease. Neuropharmacology 135:308-315 Available at: http://www.ncbi.nlm.nih.gov/pubmed/29578036.

Chen L, Liu J, Ali U, Gui ZH, Hou C, Fan LL, Wang Y, Wang T (2011) Chronic, systemic treatment with a metabotropic glutamate receptor 5 antagonist produces anxiolytic-like effects and reverses abnormal firing activity of projection neurons in the basolateral nucleus of the amygdala in rats with bilateral 6OHDA lesions. Brain Res Bull 84:215-223 Available at: http://linkinghub.elsevier.com/retrieve/pii/S0361923011000086.

Chen Y, Nong Y, Goudet C, Hemstapat K, de Paulis T, Pin J-P, Conn PJ (2007) Interaction of Novel Positive Allosteric Modulators of Metabotropic Glutamate Receptor 5 with the Negative Allosteric Antagonist Site Is Required for Potentiation of Receptor Responses. Mol Pharmacol 71:1389-1398 Available at: http://molpharm.aspetjournals.org/cgi/doi/10.1124/mol.106.03242 5.

Cheng H-C, Kim SR, Oo TF, Kareva T, Yarygina O, Rzhetskaya M, Wang C, During M, Talloczy Z, Tanaka K, Komatsu M, Kobayashi K, Okano H, Kholodilov N, Burke RE (2011) Akt suppresses retrograde degeneration of dopaminergic axons by inhibition of macroautophagy. J Neurosci 31:2125-2135 
Available at: http://www.ncbi.nlm.nih.gov/pubmed/21307249.

Cheng H, Ulane C, Burke R (2010) Clinical Progression in Parkinson's

Disease and the Neurobiology of Axons. Ann Neurol 67:715-725.

Chiang GG, Abraham RT (2005) Phosphorylation of Mammalian

Target of Rapamycin (mTOR) at Ser-2448 Is Mediated by p70S6

Kinase. J Biol Chem 280:25485-25490 Available at:

http://www.jbc.org/lookup/doi/10.1074/jbc.M501707200.

Choudhury ME, Sugimoto K, Kubo M, Nagai M, Nomoto M, Takahashi H, Yano H, Tanaka J (2011) A cytokine mixture of GM-CSF and IL-3 that induces a neuroprotective phenotype of microglia leading to amelioration of (6-OHDA)-induced Parkinsonism of rats. Brain Behav 1:26-43.

Chowdhury S, Shepherd JD, Okuno H, Lyford G, Petralia RS, Plath N, Kuhl D, Huganir RL, Worley PF (2006) Arc/Arg3.1 interacts with the endocytic machinery to regulate AMPA receptor trafficking. Neuron 52:445-459 Available at:

http://www.ncbi.nlm.nih.gov/pubmed/17088211.

Chu Y, Morfini GA, Langhamer LB, He Y, Brady ST, Kordower JH (2012) Alterations in axonal transport motor proteins in sporadic and experimental Parkinson's disease. Brain 135:2058-2073 Available at: http://www.ncbi.nlm.nih.gov/pubmed/22719003.

Chuhma N, Mingote S, Moore H, Rayport S (2014) Dopamine Neurons Control Striatal Cholinergic Neurons via Regionally 
Heterogeneous Dopamine and Glutamate Signaling. Neuron 81:901-912 Available at:

http://linkinghub.elsevier.com/retrieve/pii/S0896627313011884.

Consiglia Pacelli, Nicolas Giguère, Marie-Josée Bourque, Martin Lévesque, Ruth S. Slack TL-É (2015) Elevated mitochondrial bioenergetics and axonal arborization size are key contributors to vulnerability of dopamine neurons. Curr Biol:2349-2360.

Corti C, Battaglia G, Molinaro G, Riozzi B, Pittaluga A, Corsi M, Mugnaini M, Nicoletti F, Bruno V (2007) The Use of Knock-Out Mice Unravels Distinct Roles for mGlu2 and mGlu3 Metabotropic Glutamate Receptors in Mechanisms of Neurodegeneration/Neuroprotection. J Neurosci 27:8297-8308 Available at:

http://www.jneurosci.org/cgi/doi/10.1523/JNEUROSCI.188907.2007.

Crowley SD (2014) The Cooperative Roles of Inflammation and Oxidative Stress in the Pathogenesis of Hypertension. Antioxid Redox Signal 20:102-120 Available at: http://online.liebertpub.com/doi/abs/10.1089/ars.2013.5258.

Cunningham LA, Su C (2002) Astrocyte delivery of glial cell linederived neurotrophic factor in a mouse model of Parkinson's disease. Exp Neurol 174:230-242 Available at: http://www.ncbi.nlm.nih.gov/pubmed/11922664. 
Cunningham TJ, Yao L, Lucena A (2008) Product inhibition of secreted phospholipase A2 may explain lysophosphatidylcholines' unexpected therapeutic properties. J Inflamm (Lond) 5:17.

Dale DC, Liles WC, Llewellyn C, Price TH (1998) Effects of granulocyte-macrophage colony-stimulating factor (GM-CSF) on neutrophil kinetics and function in normal human volunteers. Am J Hematol 57:7-15.

Dave KD et al. (2014) Phenotypic characterization of recessive gene knockout rat models of Parkinson's disease. Neurobiol Dis 70:190-203 Available at: http://www.ncbi.nlm.nih.gov/pubmed/24969022.

Dave PC, Billington E, Pan Y-L, Straus SK (2005) Interaction of alamethicin with ether-linked phospholipid bilayers: oriented circular dichroism, 31P solid-state NMR, and differential scanning calorimetry studies. Biophys J 89:2434-2442.

Debeljak N, Solár P, Sytkowski AJ (2014) Erythropoietin and cancer: the unintended consequences of anemia correction. Front Immunol 5:563 Available at: http://www.pubmedcentral.nih.gov/articlerender.fcgi?artid=42275 21\&tool=pmcentrez\&rendertype $=$ abstract $[$ Accessed December $18,2014]$.

Dekundy A, Pietraszek M, Schaefer D, Cenci MA, Danysz W (2006) 
Effects of group I metabotropic glutamate receptors blockade in experimental models of Parkinson's disease. Brain Res Bull 69:318-326 Available at: http://www.ncbi.nlm.nih.gov/pubmed/16564428.

Deumens R, Blokland A, Prickaerts J (2002) Modeling Parkinson's disease in rats: an evaluation of 6-OHDA lesions of the nigrostriatal pathway. Exp Neurol 175:303-317 Available at: http://www.ncbi.nlm.nih.gov/pubmed/12061862.

Dhanushkodi A, Akano EO, Roguski EE, Xue Y, Rao SK, Matta SG, Rex TS, Mcdonald MP (2013) A single intramuscular injection of rAAV-mediated mutant erythropoietin protects against MPTPinduced parkinsonism. Genes, Brain Behav 12:224-233.

Di Maio R, Barrett PJ, Hoffman EK, Barrett CW, Zharikov A, Borah A, Hu X, McCoy J, Chu CT, Burton EA, Hastings TG, Greenamyre JT (2016) a-Synuclein binds to TOM20 and inhibits mitochondrial protein import in Parkinsons disease. Sci Transl Med 8:342ra78-342ra78 Available at: http://stm.sciencemag.org/cgi/doi/10.1126/scitranslmed.aaf3634.

Dravid A, Jaton AL, Enz A, Frei P (1984) Spontaneous recovery from motor asymmetry in adult rats with 6-hydroxydopamine-induced partial lesions of the substantia nigra. Brain Res 311:361-365 Available at: http://www.sciencedirect.com/science/article/pii/0006899384901 01X [Accessed November 27, 2017]. 
Elmer LW, Juncos JL, Singer C, Truong DD, Criswell SR, Parashos S, Felt L, Johnson R, Patni R (2018) Pooled Analyses of Phase III Studies of ADS-5102 (Amantadine) Extended-Release Capsules for Dyskinesia in Parkinson's Disease. CNS Drugs 5102 Available at: http://link.springer.com/10.1007/s40263-018-04984.

Enzler T, Dranoff G (2003) The Cytokine Handbook. Elsevier. Available at: http://www.sciencedirect.com/science/article/pii/B978012689663 3500259 [Accessed May 1, 2015].

Eriksdotter Jönhagen M, Nordberg A, Amberla K, Bäckman L, Ebendal T, Meyerson B, Olson L, Seiger Å, Shigeta M, Theodorsson E, Viitanen M, Winblad B, Wahlund L-O (1998) Intracerebroventricular Infusion of Nerve Growth Factor in Three Patients with Alzheimer's Disease. Dement Geriatr Cogn Disord 9:246-257 Available at: https://www.karger.com/Article/FullText/17069.

Estupinan D, Nathoo S, Okun MS (2013) The Demise of Poskanzer and Schwab's Influenza Theory on the Pathogenesis of Parkinson's Disease. Parkinsons Dis 2013:1-7 Available at: http://www.hindawi.com/journals/pd/2013/167843/.

Fabelo N, Martín V, Santpere G, Marín R, Torrent L, Ferrer I, Díaz M (2011) Severe alterations in lipid composition of frontal cortex lipid rafts from Parkinson's disease and incidental Parkinson's 283 
disease. Mol Med 17:1107-1118.

Factor SA, Weiner WJ (2008) Parkinson's disease diagnosis and clinical management, 2nd ed. New York: Demos Medical Publishing.

Fahn S, Oakes D, Shoulson I, Kieburtz K, Rudolph A, Lang A, Olanow C, Tanner C, Marek K, Parkinson Study Group (2004) Levodopa and the Progression of Parkinson's Disease. N Engl J Med 351:2498-2508 Available at: http://www.nejm.org/doi/abs/10.1056/NEJMoa033447.

Farmer K, Rudyk C, Prowse NA, Hayley S (2015) Hematopoietic cytokines as therapeutic players in early stages Parkinson's disease. Front Aging Neurosci.

Farrer M, Chan P, Chen R, Tan L, Lincoln S, Hernandez D, Forno L, Gwinn-Hardy K, Petrucelli L, Hussey J, Singleton A, Tanner C, Hardy J, Langston JW (2001) Lewy bodies and parkinsonism in families with parkin mutations. Ann Neurol 50:293-300.

Faull RL, Laverty R (1969) Changes in dopamine levels in the corpus striatum following lesions in the substantia nigra. Exp Neurol 23:332-340 Available at:

http://www.ncbi.nlm.nih.gov/pubmed/5767257.

Ferreira DG, Temido-Ferreira M, Miranda HV, Batalha VL, Coelho JE, Szegö ÉM, Marques-Morgado I, Vaz SH, Rhee JS, Schmitz M, Zerr I, Lopes LV., Outeiro TF. (2017) $\alpha$-synuclein interacts 
with PrPC to induce cognitive impairment through mGluR5 and NMDAR2B. Nat Neurosci 20:1569-1579 Available at:

http://www.ncbi.nlm.nih.gov/pubmed/28945221.

Ferrigno A, Vairetti M, Ambrosi G, Rizzo V, Richelmi P, Blandini F, Fuzzati-Armentero M-T (2015) Selective blockade of mGlu5 metabotropic glutamate receptors is protective against hepatic mitochondrial dysfunction in 6-OHDA lesioned Parkinsonian rats. Clin Exp Pharmacol Physiol 42:695-703 Available at: http://doi.wiley.com/10.1111/1440-1681.12410.

Fingar DC, Blenis J (2004) Target of rapamycin (TOR): an integrator of nutrient and growth factor signals and coordinator of cell growth and cell cycle progression. Oncogene 23:3151-3171 Available at: http://www.nature.com/articles/1207542.

Fonnum F (1984) Glutamate: a neurotransmitter in mammalian brain. J Neurochem 42:1-11 Available at: http://doi.wiley.com/10.1111/j.1471-4159.1984.tb09689.x.

Fox CM, Gash DM, Smoot MK, Cass WA (2001) Neuroprotective effects of GDNF against 6-OHDA in young and aged rats. Brain Res 896:56-63.

Frim DM, Uhler TA, Galpern WR, Beal MF, Breakefield XO, Isacson O (1994) Implanted fibroblasts genetically engineered to produce brain-derived neurotrophic factor prevent 1-methyl-4phenylpyridinium toxicity to dopaminergic neurons in the rat. 
Proc Natl Acad Sci U S A 91:5104-5108 Available at:

http://www.ncbi.nlm.nih.gov/pubmed/8197193.

Fuzzati-Armentero M-T, Cerri S, Levandis G, Ambrosi G, Montepeloso E, Antoninetti G, Blandini F, Baqi Y, Müller CE, Volpini R, Costa G, Simola N, Pinna A (2015) Dual target strategy: combining distinct non-dopaminergic treatments reduces neuronal cell loss and synergistically modulates 1-DOPA-induced rotational behavior in a rodent model of Parkinson's disease. J Neurochem 134:740-747 Available at: http://doi.wiley.com/10.1111/jnc.13162.

Galindo MF, Solesio ME, Atienzar-Aroca S, Zamora MJ, Jordán Bueso J (2012) Mitochondrial Dynamics and Mitophagy in the 6Hydroxydopamine Preclinical Model of Parkinson's Disease.

Parkinsons Dis 2012:1-8 Available at: http://www.hindawi.com/journals/pd/2012/131058/.

Galvan A, Wichmann T (2008) Pathophysiology of Parkinsonism. Clin Neurophysiol 119:1459-1474 Available at: http://linkinghub.elsevier.com/retrieve/pii/S1388245708001983.

Galvin JE, Uryu K, Lee VM, Trojanowski JQ (1999) Axon pathology in Parkinson' s disease and Lewy body dementia hippocampus contains alpha-, beta-, and gamma-synuclein. PNAS.

Gash DM, Zhang Z, Gerhardt G (1998) Neuroprotective and neurorestorative properties of GDNF. Ann Neurol 44:S121-S125. 
Gerber U, Gee C, Benquet P (2007) Metabotropic glutamate receptors: intracellular signaling pathways. Curr Opin Pharmacol 7:56-61 Available at: http://linkinghub.elsevier.com/retrieve/pii/S1471489206001706.

Ghosh S, Tergaonkar V, Rothlin C V., Correa RG, Bottero V, Bist P, Verma IM, Hunter T (2006) Essential role of tuberous sclerosis genes TSC1 and TSC2 in NF- $\mathrm{kB}$ activation and cell survival. Cancer Cell 10:215-226 Available at: http://linkinghub.elsevier.com/retrieve/pii/S1535610806002480.

Gill SS, Patel NK, Hotton GR, O'Sullivan K, McCarter R, Bunnage M, Brooks DJ, Svendsen CN, Heywood P (2003) Direct brain infusion of glial cell line-derived neurotrophic factor in Parkinson disease. Nat Med 9:589-595.

Giussani P, Tringali C, Riboni L, Viani P, Venerando B (2014) Sphingolipids: key regulators of apoptosis and pivotal players in cancer drug resistance. Int J Mol Sci 15:4356-4392.

Gladding CM, Fitzjohn SM, Molnar E (2009) Metabotropic Glutamate Receptor-Mediated Long-Term Depression: Molecular Mechanisms. Pharmacol Rev 61:395-412 Available at: http://pharmrev.aspetjournals.org/cgi/doi/10.1124/pr.109.001735.

Goes ATR, Souza LC, Filho CB, Fabbro L Del, De Gomes MG, Boeira SP, Jesse CR (2013) Neuroprotective effects of swimming training in a mouse model of Parkinson's disease induced by 6- 
hydroxydopamine. Neuroscience 256:61-71 Available at: http://www.ncbi.nlm.nih.gov/pubmed/24090962 [Accessed October 15, 2013].

Gökbayrak NS, Piryatinsky I, Gavett RA, Ahmed OJ (2014) Mixed Effects of Deep Brain Stimulation on Depressive Symptomatology in Parkinsonâ $\epsilon^{\mathrm{TM}} \mathrm{s}$ Disease: A Review of Randomized Clinical Trials. Front Neurol 5 Available at: http://journal.frontiersin.org/article/10.3389/fneur.2014.00154/abs tract.

Gokce O, Runne H, Kuhn A, Luthi-Carter R (2009) Short-Term Striatal Gene Expression Responses to Brain-Derived Neurotrophic Factor Are Dependent on MEK and ERK Activation. PLoS One 4:e5292.

Gonçalves I, Edsfeldt A, Ko NY, Grufman H, Berg K, Björkbacka H, Nitulescu M, Persson A, Nilsson M, Prehn C, Adamski J, Nilsson J (2012) Evidence supporting a key role of Lp-PLA2-generated lysophosphatidylcholine in human atherosclerotic plaque inflammation. Arterioscler Thromb Vasc Biol 32:1505-1512.

Goonesinghe A, Mundy ES, Smith M, Khosravi-Far R, Martinou J-C, Esposti MD (2005) Pro-apoptotic Bid induces membrane perturbation by inserting selected lysolipids into the bilayer. Biochem J 387:109-118.

Gorell JM, Johnson CC, Rybicki BA, Peterson EL, Kortsha GX, 
Brown GG, Richardson RJ (1997) Occupational exposures to metals as risk factors for Parkinson's disease. Neurology 48:650658 Available at: http://www.ncbi.nlm.nih.gov/pubmed/9065542 [Accessed December 4, 2017].

Grant LM, Kelm-Nelson CA, Hilby BL, Blue K V, Paul Rajamanickam ES, Pultorak JD, Fleming SM, Ciucci MR (2015) Evidence for early and progressive ultrasonic vocalization and oromotor deficits in a PINK1 gene knockout rat model of Parkinson's disease. J Neurosci Res 93:1713-1727 Available at: http://www.ncbi.nlm.nih.gov/pubmed/26234713.

Greenamyre JT (1986) The role of glutamate in neurotransmission and in neurologic disease. Arch Neurol 43:1058-1063.

Greenamyre JT, Porter RH (1994) Anatomy and physiology of glutamate in the CNS. Neurology 44:S7-S13.

Guimaraes IM, Carvalho TG, Ferguson SS, Pereira GS, Ribeiro FM (2015) The metabotropic glutamate receptor 5 role on motor behavior involves specific neural substrates. Mol Brain 8:24.

Gulshan K, Moye-Rowley WS (2011) Vacuolar import of phosphatidylcholine requires the ATP-binding cassette transporter Ybt1. Traffic 12:1257-1268.

Guzman JN, Sanchez-Padilla J, Chan CS, Surmeier DJ (2009) Robust Pacemaking in Substantia Nigra Dopaminergic Neurons. J Neurosci 29:11011-11019 Available at: 
http://www.jneurosci.org/cgi/doi/10.1523/JNEUROSCI.251909.2009 .

Ha Y, Kim YS, Cho JM, Yoon SH, Park SR, Yoon DH, Kim EY, Park HC (2005) Role of granulocyte-macrophage colony-stimulating factor in preventing apoptosis and improving functional outcome in experimental spinal cord contusion injury. J Neurosurg Spine 2:55-61.

Hagenah JM, König IR, Becker B, Hilker R, Kasten M, Hedrich K, Pramstaller PP, Klein C, Seidel G (2007) Substantia nigra hyperechogenicity correlates with clinical status and number of Parkin mutated alleles. J Neurol 254:1407-1413 Available at: http://www.ncbi.nlm.nih.gov/pubmed/17934880 [Accessed July $11,2014]$.

Hagg T (1998) Neurotrophins prevent death and differentially affect tyrosine hydroxylase of adult rat nigrostriatal neurons in vivo. Exp Neurol 149:183-192 Available at: http://www.ncbi.nlm.nih.gov/pubmed/9454627.

Hakimi M, Selvanantham T, Swinton E, Padmore RF, Tong Y, Kabbach G, Venderova K, Girardin SE, Bulman DE, Scherzer CR, LaVoie MJ, Gris D, Park DS, Angel JB, Shen J, Philpott DJ, Schlossmacher MG (2011) Parkinson's disease-linked LRRK2 is expressed in circulating and tissue immune cells and upregulated following recognition of microbial structures. J Neural Transm 118:795-808 Available at: 
http://www.pubmedcentral.nih.gov/articlerender.fcgi?artid=33766 $51 \&$ tool $=$ pmcentrez\&rendertype $=$ abstract $[$ Accessed January 7, 2014].

Hamilton A, Vasefi M, Tuin C Vander, Mcquaid RJ, Anisman H, Ferguson SSG (2016) Chronic Pharmacological mGluR5 Inhibition Prevents Cognitive Impairment and Reduces Pathogenesis in an Alzheimer Disease Mouse Model. Cell Rep $15: 1-7$.

Han X, Zhou D-B, Xu C-M, Yang Y, Duan M-H, Wang X, Zhang J-P, Zhao Y-Q, Shen T, Wu Y-J (2011) Effect of erythropoietin on proinflammatory factors of human monocytes and its mechanisms. J Exp Hematol / Chinese Assoc Pathophysiol 19:738-743 Available at: http://www.ncbi.nlm.nih.gov/pubmed/21729562.

Hanrott K, Gudmunsen L, O’Neill MJ, Wonnacott S (2006) 6hydroxydopamine-induced apoptosis is mediated via extracellular auto-oxidation and caspase 3-dependent activation of protein kinase Cdelta. J Biol Chem 281:5373-5382.

Hawkes CH, Del Tredici K, Braak H (2007) Parkinson's disease: A dual-hit hypothesis. Neuropathol Appl Neurobiol 33:599-614.

Hay N, Sonenberg N (2004) Upstream and downstream of mTOR. Genes Dev 18:1926-1945 Available at: http://www.genesdev.org/cgi/doi/10.1101/gad.1212704. 
Hayakawa T, Chang MCJ, Bell JM, Seeman R, Rapoport SI, Appel

NM (1998) Fatty acid incorporation depicts brain activity in a rat model of Parkinson's disease. Brain Res 807:177-181.

Henze C, Hartmann A, Lescot T, Hirsch EC, Michel PP (2005)

Proliferation of microglial cells induced by 1-methyl-4-

phenylpyridinium in mesencephalic cultures results from an

astrocyte-dependent mechanism: role of granulocyte macrophage colony-stimulating factor. J Neurochem 95:1069-1077 Available at: http://www.ncbi.nlm.nih.gov/pubmed/16135085 [Accessed June 16, 2014].

Heo HY, Park J-M, Kim C-H, Han BS, Kim K-S, Seol W (2010) LRRK2 enhances oxidative stress-induced neurotoxicity via its kinase activity. Exp Cell Res 316:649-656 Available at: http://www.ncbi.nlm.nih.gov/pubmed/19769964.

Hernandes MS, Café-Mendes CC, Britto LRG (2013a) NADPH oxidase and the degeneration of dopaminergic neurons in parkinsonian mice. Oxid Med Cell Longev 2013:157857.

Hernandes MS, Santos GDR, Café-Mendes CC, Lima LS, Scavone C, Munhoz CD, Britto LRG (2013b) Microglial Cells Are Involved in the Susceptibility of NADPH Oxidase Knockout Mice to 6Hydroxy-Dopamine-Induced Neurodegeneration. PLoS One 8:e75532.

Hirsch EC, Hunot S, Damier P, Faucheux B (1998) Glial cells and 
inflammation in Parkinson's disease: a role in neurodegeneration?

Ann Neurol 44:S115-20 Available at:

http://www.ncbi.nlm.nih.gov/pubmed/9749582.

Hisahara S, Shimohama S (2011) Dopamine Receptors and

Parkinson's Disease. Int J Med Chem 2011:1-16 Available at:

http://www.hindawi.com/journals/ijmc/2011/403039/.

Horstink M, Tolosa E, Bonuccelli U, Deuschl G, Friedman A, Kanovsky P, Larsen JP, Lees A, Oertel W, Poewe W, Rascol O, Sampaio C, European Federation of Neurological Societies, Movement Disorder Society-European Section (2006) Review of the therapeutic management of Parkinson's disease. Report of a joint task force of the European Federation of Neurological Societies and the Movement Disorder Society-European Section. Part I: early (uncomplicated) Parkinson's disease. Eur J Neurol 13:1170-1185 Available at: http://www.ncbi.nlm.nih.gov/pubmed/17038031.

Hou L, Klann E (2004) Activation of the Phosphoinositide 3-KinaseAkt-Mammalian Target of Rapamycin Signaling Pathway Is Required for Metabotropic Glutamate Receptor-Dependent LongTerm Depression. J Neurosci 24:6352-6361 Available at: http://www.jneurosci.org/cgi/doi/10.1523/JNEUROSCI.099504.2004 .

Hsieh M-H, Ho S-C, Yeh K-Y, Pawlak CR, Chang H-M, Ho Y-J, Lai T-J, Wu F-Y (2012) Blockade of metabotropic glutamate 
receptors inhibits cognition and neurodegeneration in an MPTPinduced Parkinson's disease rat model. Pharmacol Biochem Behav 102:64-71 Available at: http://linkinghub.elsevier.com/retrieve/pii/S0091305712000937.

Hu X, Li P, Guo Y, Wang H, Leak RK, Chen S, Gao Y, Chen J (2012) Microglia/macrophage polarization dynamics reveal novel mechanism of injury expansion after focal cerebral ischemia. Stroke 43:3063-3070 Available at: http://www.ncbi.nlm.nih.gov/pubmed/22933588 [Accessed December 21, 2013].

$\mathrm{Hu}$ Y (2015) The necessary role of mTORC1 in central nervous system axon regeneration. Neural Regen Res 10:186 Available at: http://www.nrronline.org/text.asp?2015/10/2/186/152363.

Huang H-Y, Lin S-Z, Kuo J-S, Chen W-F, Wang M-J (2007) G-CSF protects dopaminergic neurons from 6-OHDA-induced toxicity via the ERK pathway. Neurobiol Aging 28:1258-1269 Available at: http://www.ncbi.nlm.nih.gov/pubmed/16839644 [Accessed November 14, 2013].

Huang J, Manning BD (2009) A complex interplay between Akt, TSC2 and the two mTOR complexes: Figure 1. Biochem Soc Trans 37:217-222 Available at: http://biochemsoctrans.org/lookup/doi/10.1042/BST0370217. Hughes AJ, Daniel SE, Kilford L, Lees AJ (1992) Accuracy of clinical 
diagnosis of idiopathic Parkinson's disease: a clinico-pathological study of 100 cases. J Neurol Neurosurg Psychiatry 55:181-184 Available at: http://www.ncbi.nlm.nih.gov/pubmed/1564476.

Hung HC, Lee EH (1996) The mesolimbic dopaminergic pathway is more resistant than the nigrostriatal dopaminergic pathway to MPTP and MPP+ toxicity: role of BDNF gene expression. Brain Res Mol Brain Res 41:14-26 Available at: http://www.ncbi.nlm.nih.gov/pubmed/8883930.

Hunot S, Boissière F, Faucheux B, Brugg B, Mouatt-Prigent A, Agid Y, Hirsch EC (1996) Nitric oxide synthase and neuronal vulnerability in Parkinson's disease. Neuroscience 72:355-363 Available at: http://www.ncbi.nlm.nih.gov/pubmed/8737406.

Iderberg H, Maslava N, Thompson AD, Bubser M, Niswender CM, Hopkins CR, Lindsley CW, Conn PJ, Jones CK, Cenci MA (2015) Pharmacological stimulation of metabotropic glutamate receptor type 4 in a rat model of Parkinson's disease and $1-$ DOPA-induced dyskinesia: Comparison between a positive allosteric modulator and an orthosteric agonist. Neuropharmacology 95:121-129 Available at: http://linkinghub.elsevier.com/retrieve/pii/S0028390815000799.

Iderberg H, Rylander D, Bimpisidis Z, Cenci MA (2013) Modulating mGluR5 and 5-HT1A/1B receptors to treat 1-DOPA-induced dyskinesia: Effects of combined treatment and possible mechanisms of action. Exp Neurol 250:116-124 Available at: 
http://linkinghub.elsevier.com/retrieve/pii/S0014488613002719.

Ikenoue T, Inoki K, Yang Q, Zhou X, Guan K-L (2008) Essential function of TORC2 in PKC and Akt turn motif phosphorylation, maturation and signalling. EMBO J 27:1919-1931 Available at: http://www.ncbi.nlm.nih.gov/pubmed/18566587.

Isaacson LG, Saffran BN, Crutcher KA (1990) Intracerebral NGF infusion induces hyperinnervation of cerebral blood vessels. Neurobiol Aging 11:51-55.

Iwata S-I, Nomoto M, Morioka H, Miyata A (2004) Gene expression profiling in the midbrain of striatal 6-hydroxydopamine-injected mice. Synapse 51:279-286 Available at: http://doi.wiley.com/10.1002/syn.10307.

Jaattela M, Benedict M, Tewari M, Shayman JA, Dixit VM (1995) Bcl-x and Bcl-2 inhibit TN and Fas-induced apoptosis and activation of phospholipase A1 in breast carcinoma cells. Oncogene 10:2297-2305.

Jacinto E, Loewith R, Schmidt A, Lin S, Rüegg MA, Hall A, Hall MN (2004) Mammalian TOR complex 2 controls the actin cytoskeleton and is rapamycin insensitive. Nat Cell Biol 6:11221128 Available at:

http://www.ncbi.nlm.nih.gov/pubmed/15467718.

Jackson-Lewis V, Blesa J, Przedborski S (2012) Animal models of Parkinson's disease. Parkinsonism Relat Disord 18 Suppl 1:S183- 
5 Available at: http://www.ncbi.nlm.nih.gov/pubmed/22166429

[Accessed November 8, 2013].

Jagmag SA, Tripathi N, Shukla SD, Maiti S, Khurana S (2016)

Evaluation of Models of Parkinson's Disease. Front Neurosci 9

Available at:

http://journal.frontiersin.org/article/10.3389/fnins.2015.00503.

Jang H, Boltz D, Sturm-Ramirez K, Shepherd KR, Jiao Y, Webster R, Smeyne RJ (2009) Highly pathogenic H5N1 influenza virus can enter the central nervous system and induce neuroinflammation and neurodegeneration. Proc Natl Acad Sci U S A 106:1406314068 Available at: http://www.ncbi.nlm.nih.gov/pubmed/19667183.

Jayakar SS, Dikshit M (2004) AMPA RECEPTOR REGULATION MECHANISMS: FUTURE TARGET FOR SAFER NEUROPROTECTIVE DRUGS. Int J Neurosci 114:695-734 Available at: http://www.tandfonline.com/doi/full/10.1080/0020745049043045 3.

Jiang X, Yeung RS (2006) Regulation of microtubule-dependent protein transport by the TSC2/mammalian target of rapamycin pathway. Cancer Res 66:5258-5269 Available at: http://www.ncbi.nlm.nih.gov/pubmed/16707451.

Johnson K, Conn PJ, Niswender CM (2009) Glutamate receptors as 
therapeutic targets for Parkinson's disease. CNS Neurol Disord Drug Targets 8:475-491.

Johnston TH, Fox SH, McIldowie MJ, Piggott MJ, Brotchie JM (2010) Reduction of L-DOPA-induced dyskinesia by the selective metabotropic glutamate receptor 5 antagonist 3-[(2-methyl-1,3thiazol-4-yl)ethynyl]pyridine in the 1-methyl-4-phenyl-1,2,3,6tetrahydropyridine-lesioned macaque model of Parkinson's disease. J Pharmacol Exp Ther 333:865-873 Available at: http://jpet.aspetjournals.org/cgi/doi/10.1124/jpet.110.166629.

Jones CK, Engers DW, Thompson AD, Field JR, Blobaum AL, Lindsley SR, Zhou Y, Gogliotti RD, Jadhav S, Zamorano R, Bogenpohl J, Smith Y, Morrison R, Daniels JS, Weaver CD, Conn PJ, Lindsley CW, Niswender CM, Hopkins CR (2011) Discovery, Synthesis, and Structure-Activity Relationship Development of a Series of N -4-(2,5-Dioxopyrrolidin-1yl)phenylpicolinamides (VU0400195, ML182): Characterization of a Novel Positive Allosteric Modulator of the Metabotropic Glutamate Receptor 4. J Med Chem 54:7639-7647 Available at: http://pubs.acs.org/doi/abs/10.1021/jm200956q.

Jung CH, Ro S-H, Cao J, Otto NM, Kim D-H (2010) mTOR regulation of autophagy. FEBS Lett 584:1287-1295 Available at: http://www.ncbi.nlm.nih.gov/pubmed/20083114.

Kasper C (2003) The Cytokine Handbook. Elsevier. Available at: http://www.sciencedirect.com/science/article/pii/B978012689663 298 
3500119 [Accessed April 29, 2015].

Katzav A, Ben-Ziv T, Blank M, Pick CG, Shoenfeld Y, Chapman J (2014) Antibody-specific behavioral effects:

intracerebroventricular injection of antiphospholipid antibodies induces hyperactive behavior while anti-ribosomal-P antibodies induces depression and smell deficits in mice. J Neuroimmunol 272:10-15 Available at:

http://www.ncbi.nlm.nih.gov/pubmed/24837568 [Accessed November 13, 2014].

Kelm-Nelson CA, Yang KM, Ciucci MR (2015) Exercise Effects on Early Vocal Ultrasonic Communication Dysfunction in a PINK1 Knockout Model of Parkinson's Disease. J Parkinsons Dis 5:749763 Available at:

http://www.ncbi.nlm.nih.gov/pubmed/26577653.

Kelsey JE, Mague SD, Pijanowski RS, Harris RC, Kleckner NW, Matthews RT (2004) NMDA receptor antagonists ameliorate the stepping deficits produced by unilateral medial forebrain bundle injections of 6-OHDA in rats. Psychopharmacology (Berl) 175:179-188 Available at: http://www.ncbi.nlm.nih.gov/pubmed/15007533.

Kerner JA, Standaert DG, Penney JB, Young AB, Landwehrmeyer GB (1997) Expression of group one metabotropic glutamate receptor subunit mRNAs in neurochemically identified neurons in the rat neostriatum, neocortex, and hippocampus. Mol Brain Res 
48:259-269 Available at:

http://linkinghub.elsevier.com/retrieve/pii/S0169328X97001022.

Khan MM, Ahmad A, Ishrat T, Khan MB, Hoda MN, Khuwaja G, Raza SS, Khan A, Javed H, Vaibhav K, Islam F (2010)

Resveratrol attenuates 6-hydroxydopamine-induced oxidative damage and dopamine depletion in rat model of Parkinson's disease. Brain Res 1328:139-151.

Kim JK, Choi BH, Park HC, Park SR, Kim YS, Yoon SH, Park HS, Kim EY, Ha Y (2004) Effects of GM-CSF on the neural progenitor cells. Neuroreport 15:2161-2165 Available at: http://content.wkhealth.com/linkback/openurl?sid=WKPTLP:land ingpage $\& a n=00001756-200410050-00003$.

Kim NK, Choi BH, Huang X, Snyder BJ, Bukhari S, Kong T-H, Park H, Park HC, Park SR, Ha Y (2009a) Granulocyte-macrophage colony-stimulating factor promotes survival of dopaminergic neurons in the 1-methyl-4-phenyl-1,2,3,6-tetrahydropyridineinduced murine Parkinson's disease model. Eur J Neurosci 29:891-900 Available at: http://www.ncbi.nlm.nih.gov/pubmed/19245369 [Accessed November 13, 2013].

Kim SJ, Gershov D, Ma X, Brot N, Elkon KB (2002) I-PLA2 Activation during Apoptosis Promotes the Exposure of Membrane Lysophosphatidylcholine Leading to Binding by Natural Immunoglobulin M Antibodies and Complement 
Activation. J Exp Med 196:655-665.

Kim SR, Chen X, Oo TF, Kareva T, Yarygina O, Wang C, During M, Kholodilov N, Burke RE (2011) Dopaminergic pathway reconstruction by Akt/Rheb-induced axon regeneration. Ann Neurol 70:110-120 Available at: http://www.ncbi.nlm.nih.gov/pubmed/21437936.

Kim SR, Kareva T, Yarygina O, Kholodilov N, Burke RE (2009b) AAV Transduction of Dopamine Neurons With Constitutively Active Rheb Protects From Neurodegeneration and Mediates Axon Regrowth. Mol Ther 20:275-286 Available at: http://dx.doi.org/10.1038/mt.2011.213/nature06264.

Kim SR, Kareva T, Yarygina O, Kholodilov N, Burke RE (2012) AAV transduction of dopamine neurons with constitutively active Rheb protects from neurodegeneration and mediates axon regrowth. Mol Ther 20:275-286 Available at: http://www.ncbi.nlm.nih.gov/pubmed/22008911.

Kim WS, Kågedal K, Halliday GM (2014) Alpha-synuclein biology in Lewy body diseases. Alzheimers Res Ther 6:73 Available at: http://alzres.biomedcentral.com/articles/10.1186/s13195-0140073-2.

Kirik D, Rosenblad C, Björklund a (1998) Characterization of behavioral and neurodegenerative changes following partial lesions of the nigrostriatal dopamine system induced by 
intrastriatal 6-hydroxydopamine in the rat. Exp Neurol 152:259-

277 Available at: http://www.ncbi.nlm.nih.gov/pubmed/9710526.

Klockgether T (2004) Parkinson's disease: clinical aspects. Cell Tissue Res 318:115-120.

Klockgether T, Wüllner U, Steinbach JP, Petersen V, Turski L, Löschmann PA (1996) Effects of the antiparkinsonian drug budipine on central neurotransmitter systems. Eur J Pharmacol 301:67-73 Available at: http://www.ncbi.nlm.nih.gov/pubmed/8773448.

Ko WKD, Pioli E, Li Q, McGuire S, Dufour A, Sherer TB, Bezard E, Facheris MF (2014) Combined fenobam and amantadine treatment promotes robust antidyskinetic effects in the 1-methyl4-phenyl-1,2,3,6-tetrahydropyridine (MPTP)-lesioned primate model of Parkinson's disease. Mov Disord 29:772-779 Available at: http://doi.wiley.com/10.1002/mds.25859.

Kohno R, Sawada H, Kawamoto Y, Uemura K, Shibasaki H, Shimohama S (2004) BDNF is induced by wild-type alphasynuclein but not by the two mutants, A30P or A53T, in glioma cell line. Biochem Biophys Res Commun 318:113-118 Available at: http://www.ncbi.nlm.nih.gov/pubmed/15110760.

Kolbeck R, Bartke I, Eberle W, Barde YA (1999) Brain-derived neurotrophic factor levels in the nervous system of wild-type and neurotrophin gene mutant mice. J Neurochem 72:1930-1938 
Available at: http://www.ncbi.nlm.nih.gov/pubmed/10217270.

Kosloski LM, Kosmacek EA, Olson KE, Mosley RL, Gendelman HE (2013) GM-CSF induces neuroprotective and anti-inflammatory responses in 1-methyl-4-phenyl-1,2,3,6-tetrahydropyridine intoxicated mice. J Neuroimmunol 265:1-10 Available at: http://www.ncbi.nlm.nih.gov/pubmed/24210793 [Accessed November 12, 2013].

Kreisler A, Gelé P, Wiart J-F, Lhermitte M, Destée A, Bordet R (2007) Lipid-lowering drugs in the MPTP mouse model of Parkinson's disease: fenofibrate has a neuroprotective effect, whereas bezafibrate and HMG-CoA reductase inhibitors do not. Brain Res 1135:77-84.

Krüger R, Kuhn W, Müller T, Woitalla D, Graeber M, Kösel S, Przuntek H, Epplen JT, Schols L, Riess O (1998) AlaSOPro mutation in the gene encoding $\alpha$-synuclein in Parkinson's disease. Nat Genet 18:106-108 Available at: http://www.nature.com/doifinder/10.1038/ng0298-106.

Kumar R, Agarwal AK, Seth PK (1995) Free radical-generated neurotoxicity of 6-hydroxydopamine. J Neurochem 64:17031707 Available at: http://www.ncbi.nlm.nih.gov/pubmed/7891098.

Kumar V, Fahey PG, Jong Y-JI, Ramanan N, O’Malley KL (2012) Activation of Intracellular Metabotropic Glutamate Receptor 5 in 
Striatal Neurons Leads to Up-regulation of Genes Associated with Sustained Synaptic Transmission Including Arc/Arg3.1 Protein. J Biol Chem 287:5412-5425.

Kupsch A, Schmidt W, Gizatullina Z, Debska-Vielhaber G, Voges J, Striggow F, Panther P, Schwegler H, Heinze H-J, Vielhaber S, Gellerich FN (2014) 6-Hydroxydopamine impairs mitochondrial function in the rat model of Parkinson's disease: respirometric, histological, and behavioral analyses. J Neural Transm 121:12451257 Available at:

http://www.ncbi.nlm.nih.gov/pubmed/24627045 [Accessed March 17, 2014].

Lan A, Chen J, Zhao Y, Chai Z, Hu Y (2016) mTOR Signaling in Parkinson's Disease. NeuroMolecular Med Available at: http://link.springer.com/10.1007/s12017-016-8417-7.

Lang AE et al. (2006) Randomized controlled trial of intraputamenal glial cell line-derived neurotrophic factor infusion in Parkinson disease. Ann Neurol 59:459-466.

Lapchak PA, Beck KD, Araujo DM, Irwin I, Langston JW, Hefti F (1993) Chronic intranigral administration of brain-derived neurotrophic factor produces striatal dopaminergic hypofunction in unlesioned adult rats and fails to attenuate the decline of striatal dopaminergic function following medial forebrain bundle transection. Neuroscience 53:639-650 Available at: http://www.ncbi.nlm.nih.gov/pubmed/8098137. 
Laplante M, Sabatini DM (2009) An Emerging Role of mTOR in Lipid Biosynthesis. Curr Biol 19:R1046-R1052 Available at: http://linkinghub.elsevier.com/retrieve/pii/S0960982209017710.

Lau YS, Hao R, Fung YK, Fu LS, Bishop JF, Pfeiffer RF, Mouradian MM (1998) Modulation of nigrostriatal dopaminergic transmission by antisense oligodeoxynucleotide against brainderived neurotrophic factor. Neurochem Res 23:525-532 Available at: http://www.ncbi.nlm.nih.gov/pubmed/9566587.

Le Poul E, Bolea C, Girard F, Poli S, Charvin D, Campo B, Bortoli J, Bessif A, Luo B, Koser AJ, Hodge LM, Smith KM, DiLella AG, Liverton N, Hess F, Browne SE, Reynolds IJ (2012) A Potent and Selective Metabotropic Glutamate Receptor 4 Positive Allosteric Modulator Improves Movement in Rodent Models of Parkinson's Disease. J Pharmacol Exp Ther 343:167-177 Available at: http://jpet.aspetjournals.org/cgi/doi/10.1124/jpet.112.196063.

Le W, Sayana P, Jankovic J (2013) Animal Models of Parkinson's Disease: A Gateway to Therapeutics? Neurotherapeutics Available at: http://www.ncbi.nlm.nih.gov/pubmed/24158912 [Accessed November 11, 2013].

Lee CS, Sauer H, Bjorklund A (1996) Dopaminergic neuronal degeneration and motor impairments following axon terminal lesion by instrastriatal 6-hydroxydopamine in the rat. Neuroscience 72:641-653 Available at: http://www.ncbi.nlm.nih.gov/pubmed/9157311. 
Lee H-J, Bazinet RP, Rapoport SI, Bhattacharjee AK (2010) Brain arachidonic acid cascade enzymes are upregulated in a rat model of unilateral parkinson disease. Neurochem Res 35:613-619.

Lee S, Margolin K (2011) Cytokines in Cancer Immunotherapy. Cancers (Basel) 3:3856-3893 Available at: http:/www.mdpi.com/2072-6694/3/4/3856/.

Lefloch R, Pouysségur J, Lenormand P (2009) Total ERK1/2 activity regulates cell proliferation. Cell Cycle 8:705-711 Available at: http://www.ncbi.nlm.nih.gov/pubmed/19242111.

Lehrmann E, Christensen T, Zimmer J, Diemer NH, Finsen B (1997) Microglial and macrophage reactions mark progressive changes and define the penumbra in the rat neocortex and striatum after transient middle cerebral artery occlusion. J Comp Neurol $386: 461-476$.

Levivier M, Przedborski S, Bencsics C, Kang UJ (1995) Intrastriatal implantation of fibroblasts genetically engineered to produce brain-derived neurotrophic factor prevents degeneration of dopaminergic neurons in a rat model of Parkinson's disease. J Neurosci 15:7810-7820 Available at: http://www.ncbi.nlm.nih.gov/pubmed/8613721.

Li Y, Inoki K, Guan K-L (2004) Biochemical and functional characterizations of small GTPase Rheb and TSC2 GAP activity. Mol Cell Biol 24:7965-7975 Available at: 
http://www.ncbi.nlm.nih.gov/pubmed/15340059.

Lin E, Cavanaugh JE, Leak RK, Perez RG, Zigmond MJ (2008) Rapid activation of ERK by 6-hydroxydopamine promotes survival of dopaminergic cells. J Neurosci Res 86:108-117 Available at: http://www.ncbi.nlm.nih.gov/pubmed/17847117.

Lindemann L et al. (2015) Pharmacology of basimglurant (RO4917523, RG7090), a unique metabotropic glutamate receptor 5 negative allosteric modulator in clinical development for depression. J Pharmacol Exp Ther 353:213-233.

Lindemann L, Jaeschke G, Michalon A, Vieira E, Honer M, Spooren W, Porter R, Hartung T, Kolczewski S, Buttelmann B, Flament C, Diener C, Fischer C, Gatti S, Prinssen EP, Parrott N, Hoffmann G, Wettstein JG (2011) CTEP: A Novel, Potent, Long-Acting, and Orally Bioavailable Metabotropic Glutamate Receptor 5 Inhibitor. J Pharmacol Exp Ther 339:474-486.

Ling Z, Zhu Y, Tong C wai, Snyder JA, Lipton JW, Carvey PM (2006) Progressive dopamine neuron loss following supra-nigral lipopolysaccharide (LPS) infusion into rats exposed to LPS prenatally. Exp Neurol 199:499-512 Available at: http://linkinghub.elsevier.com/retrieve/pii/S0014488606000185.

Litteljohn D, Hayley S (2012) Cytokines as potential biomarkers for Parkinson's disease: A multiplex approach (Yan Q, ed). Totowa, NJ: Humana Press. Available at: 
http://link.springer.com/10.1007/978-1-62703-071-7.

Litteljohn D, Rudyk C, Dwyer Z, Farmer K, Fortin T, Hayley S (2017) The impact of murine LRRK2 G2019S transgene overexpression on acute responses to inflammatory challenge. Brain Behav Immun.

Liu-Wu Y, Hurt-Camejo E, Wiklund O (1998) Lysophosphatidylcholine induces the production of IL-1beta by human monocytes. Atherosclerosis 137:351-357.

Lohmeyer M, Bittman R (1994) Antitumore ether lipids and alkylphosphocholines. Drugs Future 19:1021-1037.

Lopez S, Bonito-Oliva A, Pallottino S, Acher F, Fisone G (2011) Activation of metabotropic glutamate 4 receptors decreases LDOPA-induced dyskinesia in a mouse model of Parkinson's disease. J Parkinsons Dis 1:339-346 Available at: http://www.ncbi.nlm.nih.gov/pubmed/23939343.

Lopez S, Jouve L, Turle-Lorenzo N, Kerkerian-LeGoff L, Salin P, Amalric M (2012) Antiparkinsonian action of a selective group III mGlu receptor agonist is associated with reversal of subthalamonigral overactivity. Neurobiol Dis 46:69-77 Available at: http://linkinghub.elsevier.com/retrieve/pii/S0969996111004311.

Löschmann PA, Lange KW, Kunow M, Rettig KJ, Jähnig P, Honoré T, Turski L, Wachtel H, Jenner P, Marsden CD (1991) Synergism of 308 
the AMPA-antagonist NBQX and the NMDA-antagonist CPP with L-dopa in models of Parkinson's disease. J Neural Transm Park Dis Dement Sect 3:203-213 Available at: http://www.ncbi.nlm.nih.gov/pubmed/1835581.

Lotharius J, Brundin P (2002) Pathogenesis of Parkinson's disease: dopamine, vesicles and alpha-synuclein. Nat Rev Neurosci 3:932-942 Available at: http://www.ncbi.nlm.nih.gov/pubmed/12461550 [Accessed July $11,2014]$.

Love S, Plaha P, Patel NK, Hotton GR, Brooks DJ, Gill SS (2005) Glial cell line-derived neurotrophic factor induces neuronal sprouting in human brain.

Lundblad M, Picconi B, Lindgren H, Cenci MA (2004) A model of LDOPA-induced dyskinesia in 6-hydroxydopamine lesioned mice: relation to motor and cellular parameters of nigrostriatal function. Neurobiol Dis 16:110-123 Available at: http://www.ncbi.nlm.nih.gov/pubmed/15207268.

Ma Y, Galluzzi L, Zitvogel L, Kroemer G (2013) Autophagy and Cellular Immune Responses. Immunity 39:211-227 Available at: http://linkinghub.elsevier.com/retrieve/pii/S1074761313003257.

Maiese K (2015) mTOR: Driving apoptosis and autophagy for neurocardiac complications of diabetes mellitus. World J Diabetes 6:217 Available at: http://www.wjgnet.com/1948- 
9358/full/v6/i2/217.htm.

Maiese K (2016) Targeting molecules to medicine with mTOR, autophagy and neurodegenerative disorders. Br J Clin Pharmacol 82:1245-1266 Available at: http://doi.wiley.com/10.1111/bcp.12804.

Mangano EN, Hayley S (2009) Inflammatory priming of the substantia nigra influences the impact of later paraquat exposure: Neuroimmune sensitization of neurodegeneration. Neurobiol Aging 30:1361-1378 Available at: http://www.ncbi.nlm.nih.gov/pubmed/18187236 [Accessed November 14, 2013].

Mangano EN, Peters S, Litteljohn D, So R, Bethune C, Bobyn J, Clarke M, Hayley S (2011) Granulocyte macrophage-colony stimulating factor protects against substantia nigra dopaminergic cell loss in an environmental toxin model of Parkinson's disease. Neurobiol Dis 43:99-112 Available at: http://www.ncbi.nlm.nih.gov/pubmed/21377529 [Accessed April 29, 2013].

Manning-Bog AB, McCormack AL, Li J, Uversky VN, Fink AL, Di Monte DA (2002) The herbicide paraquat causes up-regulation and aggregation of alpha-synuclein in mice: paraquat and alphasynuclein. J Biol Chem 277:1641-1644 Available at: http://www.ncbi.nlm.nih.gov/pubmed/11707429. 
Manzoni C, Lewis PA (2017) LRRK2 and Autophagy. Adv Neurobiol 14:89-105 Available at:

http:/www.ncbi.nlm.nih.gov/pubmed/28353280.

Mapstone M, Cheema AK, Fiandaca MS, Zhong X, Mhyre TR, MacArthur LH, Hall WJ, Fisher SG, Peterson DR, Haley JM, Nazar MD, Rich S a, Berlau DJ, Peltz CB, Tan MT, Kawas CH, Federoff HJ (2014) Plasma phospholipids identify antecedent memory impairment in older adults. Nat Med 20:415-418 Available at: http://www.ncbi.nlm.nih.gov/pubmed/24608097 [Accessed July 9, 2014].

Maranis S, Stamatis D, Tsironis C, Konitsiotis S (2012) Investigation of the antidyskinetic site of action of metabotropic and ionotropic glutamate receptor antagonists. Intracerebral infusions in 6hydroxydopamine-lesioned rats with levodopa-induced dyskinesia. Eur J Pharmacol 683:71-77 Available at: http://linkinghub.elsevier.com/retrieve/pii/S0014299912001999.

Marié R, Barré L, Dupuy B, Viader F, Defer G, Baron J (1999) Relationships between striatal dopamine denervation and frontal executive tests in Parkinson's disease. Neurosci Lett 260:77-80.

Marin C, Bonastre M, Aguilar E, Jiménez A (2011) The metabotropic glutamate receptor antagonist 2-methyl-6-(phenylethynyl) pyridine decreases striatal VGlut2 expression in association with an attenuation of L-dopa-induced dyskinesias. Synapse 65:10801086 Available at: http://doi.wiley.com/10.1002/syn.20941. 
Marinova-Mutafchieva L, Sadeghian M, Broom L, Davis JB, Medhurst AD, Dexter DT (2009) Relationship between microglial activation and dopaminergic neuronal loss in the substantia nigra: a time course study in a 6-hydroxydopamine model of Parkinson's disease. J Neurochem 110:966-975 Available at: http://www.ncbi.nlm.nih.gov/pubmed/19549006 [Accessed November 5, 2013].

Martin-Iverson MT, Altar CA (1996) Spontaneous Behaviours of Rats are Differentially Affected by Substantia Nigra Infusions of Brain-derived Neurotrophic Factor and Neurotrophin-3. Eur J Neurosci 8:1696-1706 Available at: http://doi.wiley.com/10.1111/j.1460-9568.1996.tb01313.x.

Martin-Iverson MT, Todd KG, Altar CA (1994) Brain-derived neurotrophic factor and neurotrophin-3 activate striatal dopamine and serotonin metabolism and related behaviors: interactions with amphetamine. J Neurosci 14:1262-1270 Available at: http://www.ncbi.nlm.nih.gov/pubmed/8074747.

Masilamoni GJ, Bogenpohl JW, Alagille D, Delevich K, Tamagnan G, Votaw JR, Wichmann T, Smith Y (2011) Metabotropic glutamate receptor 5 antagonist protects dopaminergic and noradrenergic neurons from degeneration in MPTP-treated monkeys. Brain 134:2057-2073 Available at:

https://academic.oup.com/brain/articlelookup/doi/10.1093/brain/awr137. 
Mattock C, Marmot M, Stern G (1988) Could Parkinson\&\#039;s disease follow intra-uterine influenza?: a speculative hypothesis. J Neurol Neurosurg \&amp;amp; Psychiatry 51:753 LP-756 Available at: http://jnnp.bmj.com/content/51/6/753.abstract.

Mayer C, Grummt I (2006) Ribosome biogenesis and cell growth: mTOR coordinates transcription by all three classes of nuclear RNA polymerases. Oncogene 25:6384-6391 Available at: http://www.nature.com/articles/1209883.

McDonald WM, Richard IH, DeLong MR (2003) Prevalence, etiology, and treatment of depression in Parkinson's disease. Biol Psychiatry 54:363-375.

McLay RN, Banks WA, Kastin AJ (1997) Granulocyte macrophagecolony stimulating factor crosses the blood-testis barrier in mice. Biol Reprod 57:822-826.

McMurray HF, Parthasarathy S, Steinberg D (1993) Oxidatively modified low density lipoprotein is a chemoattractant for human T lymphocytes. J Clin Invest 92:1004-1008.

Mehler-Wex C, Riederer P, Gerlach M (2006) Dopaminergic dysbalance in distinct basal ganglia neurocircuits: implications for the pathophysiology of Parkinson's disease, schizophrenia and attention deficit hyperactivity disorder. Neurotox Res 10:167179.

Mengozzi M, Cervellini I, Villa P, Erbayraktar Z, Gökmen N, Yilmaz 
O, Erbayraktar S, Manohasandra M, Van Hummelen P, Vandenabeele P, Chernajovsky Y, Annenkov A, Ghezzi P (2012) Erythropoietin-induced changes in brain gene expression reveal induction of synaptic plasticity genes in experimental stroke. Proc Natl Acad Sci U S A 109:9617-9622 Available at:

http://dx.doi.org/10.10710.1073/pnas.1200554109.

Menza M, Donkin R, Marin H (2006) Treatment of depression in Parkinson's disease. Curr Psychiatry Rep 8:234-240.

Merelli a, Czornyj L, Lazarowski a (2014) Erythropoietin as a new therapeutic opportunity in brain inflammation and neurodegenrreative diseases. Int J Neurosci:1-11 Available at: http://www.ncbi.nlm.nih.gov/pubmed/25405533 [Accessed January 8, 2015].

Merelli A, Czornyj L, Lazarowski A (2013) Erythropoietin: a neuroprotective agent in cerebral hypoxia, neurodegeneration, and epilepsy. Curr Pharm Des 19:6791-6801.

Merelli A, Czornyj L, Lazarowski A (2015) Erythropoietin as a new therapeutic opportunity in brain inflammation and neurodegenerative diseases. Int J Neurosci:150109023229008 Available at:

http://informahealthcare.com/doi/abs/10.3109/00207454.2014.98 9321.

Michalon A, Sidorov M, Ballard TM, Ozmen L, Spooren W, Wettstein 
JG, Jaeschke G, Bear MF, Lindemann L (2012) Chronic

Pharmacological mGlu5 Inhibition Corrects Fragile X in Adult Mice. Neuron 74:49-56.

Micheli F (2000) Methylphenylethynylpyridine (MPEP) Novartis. Curr Opin Investig Drugs 1:355-359 Available at: http://www.ncbi.nlm.nih.gov/pubmed/11249719.

Mielke MM, Maetzler W, Haughey NJ, Bandaru VVR, Savica R, Deuschle C, Gasser T, Hauser A-K, Gräber-Sultan S, Schleicher E, Berg D, Liepelt-Scarfone I (2013) Plasma ceramide and glucosylceramide metabolism is altered in sporadic Parkinson's disease and associated with cognitive impairment: a pilot study. PLoS One 8:e73094 Available at: http://www.pubmedcentral.nih.gov/articlerender.fcgi? artid=37768 17\&tool=pmcentrez\&rendertype=abstract [Accessed July 11, 2014].

Miller DB, O’Callaghan JP (2015) Biomarkers of Parkinson's disease: Present and future. Metabolism 64:S40-S46 Available at: http://linkinghub.elsevier.com/retrieve/pii/S0026049514003394.

Mitsumoto Y, Watanabe A, Mori A, Koga N (1998) Spontaneous Regeneration of Nigrostriatal Dopaminergic Neurons in MPTPTreated C57BL/6 Mice. Biochem Biophys Res Commun 248:660-663 Available at: http://www.ncbi.nlm.nih.gov/pubmed/9703982 [Accessed November 27, 2017]. 
Mogi M, Togari A, Kondo T, Mizuno Y, Komure O, Kuno S, Ichinose H, Nagatsu T (1999) Brain-derived growth factor and nerve growth factor concentrations are decreased in the substantia nigra in Parkinson's disease. Neurosci Lett 270:45-48.

Moisan F, Spinosi J, Dupupet J-L, Delabre L, Mazurie J-L, Goldberg M, Imbernon E, Tzourio C, Elbaz A (2011) The relation between type of farming and prevalence of Parkinson's disease among agricultural workers in five French districts. Mov Disord 26:271279 Available at: http://www.ncbi.nlm.nih.gov/pubmed/21412834 [Accessed December 4, 2017].

Monaghan DT, Irvine MW, Costa BM, Fang G, Jane DE (2012) Pharmacological modulation of NMDA receptor activity and the advent of negative and positive allosteric modulators. Neurochem Int 61:581-592 Available at: http://linkinghub.elsevier.com/retrieve/pii/S019701861200006X.

Morin N, Grégoire L, Gomez-Mancilla B, Gasparini F, Di Paolo T (2010) Effect of the metabotropic glutamate receptor type 5 antagonists MPEP and MTEP in parkinsonian monkeys. Neuropharmacology 58:981-986 Available at: http://linkinghub.elsevier.com/retrieve/pii/S002839081000002X.

Morin N, Grégoire L, Morissette M, Desrayaud S, Gomez-Mancilla B, Gasparini F, Di Paolo T (2013a) MPEP, an mGlu5 receptor antagonist, reduces the development of 1-DOPA-induced motor complications in de novo parkinsonian monkeys: Biochemical 
correlates. Neuropharmacology 66:355-364 Available at: http://linkinghub.elsevier.com/retrieve/pii/S0028390812003851.

Morin N, Jourdain VA, Morissette M, Grégoire L, Di Paolo T (2014) Long-term treatment with 1-DOPA and an mGlu5 receptor antagonist prevents changes in brain basal ganglia dopamine receptors, their associated signaling proteins and neuropeptides in parkinsonian monkeys. Neuropharmacology 79:688-706 Available at: http://linkinghub.elsevier.com/retrieve/pii/S0028390814000215.

Morin N, Morissette M, Grégoire L, Di T (2016) mGlu5 , Dopamine D 2 and Adenosine A 2A Receptors in L-DOPA-induced Dyskinesias. :481-493.

Morin N, Morissette M, Grégoire L, Gomez-Mancilla B, Gasparini F, Di Paolo T (2013b) Chronic treatment with MPEP, an mGlu5 receptor antagonist, normalizes basal ganglia glutamate neurotransmission in 1-DOPA-treated parkinsonian monkeys. Neuropharmacology 73:216-231 Available at: http://linkinghub.elsevier.com/retrieve/pii/S0028390813002426.

Morita M, Gravel S-P, Chénard V, Sikström K, Zheng L, Alain T, Gandin V, Avizonis D, Arguello M, Zakaria C, McLaughlan S, Nouet Y, Pause A, Pollak M, Gottlieb E, Larsson O, St-Pierre J, Topisirovic I, Sonenberg N (2013) mTORC1 Controls Mitochondrial Activity and Biogenesis through 4E-BP-Dependent Translational Regulation. Cell Metab 18:698-711 Available at: 
http://linkinghub.elsevier.com/retrieve/pii/S1550413113004130.

Moult PR, Corrêa SAL, Collingridge GL, Fitzjohn SM, Bashir ZI (2008) Co-activation of p38 mitogen-activated protein kinase and protein tyrosine phosphatase underlies metabotropic glutamate receptor-dependent long-term depression. J Physiol 586:24992510 Available at:

http://www.ncbi.nlm.nih.gov/pubmed/18356198.

Murer MG, Yan Q, Raisman-Vozari R (2001) Brain-derived neurotrophic factor in the control human brain, and in Alzheimer's disease and Parkinson's disease. Prog Neurobiol 63:71-124.

Nagatsu T, Mogi M, Ichinose H, Togari A (2000) Cytokines in Parkinson's disease. J Neural Transm Suppl:143-151 Available at: http://www.ncbi.nlm.nih.gov/pubmed/11128604.

Nakagawa M, Inokuchi M, Takagi Y, Kato K, Sugita H, Otsuki S, Kojima K, Uetake H, Sugihara K (2014) ErythropoietinProducing Hepatocellular A1 is an Independent Prognostic Factor for Gastric Cancer. Ann Surg Oncol Available at: http://www.ncbi.nlm.nih.gov/pubmed/25391265 [Accessed January 8, 2015].

Nakagawa T, Suga S, Kawase T, Toda M (2006) Intracarotid injection of granulocyte-macrophage colony-stimulating factor induces neuroprotection in a rat transient middle cerebral artery occlusion 
model. Brain Res 1089:179-185.

Nicoletti F, Bockaert J, Collingridge GL, Conn PJ, Ferraguti F, Schoepp DD, Wroblewski JT, Pin JP (2011) Metabotropic glutamate receptors: From the workbench to the bedside. Neuropharmacology 60:1017-1041 Available at: http://linkinghub.elsevier.com/retrieve/pii/S0028390810002923.

Nishimura F, Yoshikawa M, Kanda S, Nonaka M, Yokota H, Shiroi A, Nakase H, Hirabayashi H, Ouji Y, Birumachi J-I, Ishizaka S, Sakaki T (2003) Potential use of embryonic stem cells for the treatment of mouse parkinsonian models: improved behavior by transplantation of in vitro differentiated dopaminergic neurons from embryonic stem cells. Stem Cells 21:171-180 Available at: http://www.ncbi.nlm.nih.gov/pubmed/12634413.

Nunez J (2008) Morris Water Maze Experiment. J Vis Exp.

O'Brien JA (2004) A Novel Selective Allosteric Modulator Potentiates the Activity of Native Metabotropic Glutamate Receptor Subtype 5 in Rat Forebrain. J Pharmacol Exp Ther 309:568-577 Available at: http://jpet.aspetjournals.org/cgi/doi/10.1124/jpet.103.061747.

Okun M, Fernandez H, Grosset D, Grosset K (2009) Parkinson's disease, 1st ed. London: CBC Press.

Osaka H, Wang Y-L, Takada K, Takizawa S, Setsuie R, Li H, Sato Y, Nishikawa K, Sun Y-J, Sakurai M, Harada T, Hara Y, Kimura I, Chiba S, Namikawa K, Kiyama H, Noda M, Aoki S, Wada K 
(2003) Ubiquitin carboxy-terminal hydrolase L1 binds to and stabilizes monoubiquitin in neuron. Hum Mol Genet 12:19451958 Available at:

http://www.ncbi.nlm.nih.gov/pubmed/12913066.

Osterweil EK, Krueger DD, Reinhold K, Bear MF (2010) Hypersensitivity to mGluR5 and ERK1/2 Leads to Excessive Protein Synthesis in the Hippocampus of a Mouse Model of Fragile X Syndrome. J Neurosci 30:15616-15627 Available at: http://www.jneurosci.org/cgi/doi/10.1523/JNEUROSCI.388810.2010 .

Parajuli B, Sonobe Y, Kawanokuchi J, Doi Y, Noda M, Takeuchi H, Mizuno T, Suzumura A (2012) GM-CSF increases LPS-induced production of proinflammatory mediators via upregulation of TLR4 and CD14 in murine microglia. J Neuroinflammation 9:268 Available at:

http://www.pubmedcentral.nih.gov/articlerender.fcgi?artid=35659 88\&tool=pmcentrez\&rendertype $=$ abstract [Accessed January 6, 2014].

Park SY, Kim DY, Kang J-K, Park G, Choi Y-W (2014) Involvement of activation of the Nrf2/ARE pathway in protection against 6OHDA-induced SH-SY5Y cell death by $\alpha$-iso-cubebenol. Neurotoxicology:1-9 Available at: http://www.ncbi.nlm.nih.gov/pubmed/24997245 [Accessed July 7, 2014]. 
Parkinson Study Group (2000) Pramipexole vs Levodopa as Initial Treatment for Parkinson Disease. JAMA 284:1931 Available at: http://jama.jamanetwork.com/article.aspx?doi=10.1001/jama.284. 15.1931 .

Paxinos G, Watson C (1997) The Rat Brain in Stereotaxic Coordinates, 3rd ed.

Peebles CL, Yoo J, Thwin MT, Palop JJ, Noebels JL, Finkbeiner S (2010) Arc regulates spine morphology and maintains network stability in vivo. Proc Natl Acad Sci U S A 107:18173-18178 Available at: http://www.ncbi.nlm.nih.gov/pubmed/20921410.

Pellicena P, Schulman H (2014) CaMKII inhibitors: from research tools to therapeutic agents. Front Pharmacol 5:1-10 Available at: http://journal.frontiersin.org/article/10.3389/fphar.2014.00021/abs tract.

Perier C, Bové J, Wu D-C, Dehay B, Choi D-K, Jackson-Lewis V, Rathke-Hartlieb S, Bouillet P, Strasser A, Schulz JB, Przedborski S, Vila M (2007) Two molecular pathways initiate mitochondriadependent dopaminergic neurodegeneration in experimental Parkinson's disease. Proc Natl Acad Sci U S A 104:8161-8166 Available at: http://www.pubmedcentral.nih.gov/articlerender.fcgi?artid=18765 $88 \&$ tool $=$ pmcentrez\&rendertype $=$ abstract.

Pezet S, McMahon SB (2006) Neurotrophins: mediators and 
modulators of pain. Annu Rev Neurosci 29:507-538.

Pilakka-Kanthikeel S, Atluri VSR, Sagar V, Nair M (2013) Targeted Brain Derived Neurotropic Factors (BDNF) Delivery across the Blood-Brain Barrier for Neuro-Protection Using Magnetic Nano Carriers: An In-Vitro Study. PLoS One 8.

Poli A, Gandolfi O, Lucchi R, Barnabei O (1992) Spontaneous recovery of MPTP-damaged catecholamine systems in goldfish brain areas. Brain Res 585:128-134 Available at: http://www.sciencedirect.com/science/article/pii/0006899392911 97M?via\%3Dihub [Accessed November 27, 2017].

Polymeropoulos MH et al. (1997) Mutation in the alpha-synuclein gene identified in families with Parkinson's disease. Science 276:2045-2047 Available at:

http://www.ncbi.nlm.nih.gov/pubmed/9197268.

Pop AS, Gomez-Mancilla B, Neri G, Willemsen R, Gasparini F (2014) Fragile X syndrome: a preclinical review on metabotropic glutamate receptor 5 (mGluR5) antagonists and drug development. Psychopharmacology (Berl) 231:1217-1226 Available at: http://www.ncbi.nlm.nih.gov/pubmed/24232444.

Poskanzer D, Schwab R (1961) Studies in the epidemiology of Parkinson's disease predicting its disappearance as a major clinical entity by 1980. Trans Am Neurol Assoc 86:234-235 Available at: http://www.ncbi.nlm.nih.gov/pubmed/14488169. 
Prasad K, Winnik B, Thiruchelvam MJ, Buckley B, Mirochnitchenko O, Richfield EK (2007) Prolonged toxicokinetics and toxicodynamics of paraquat in mouse brain. Environ Health Perspect 115:1448-1453 Available at:

http://www.pubmedcentral.nih.gov/articlerender.fcgi?artid=20226 43\&tool=pmcentrez\&rendertype $=$ abstract [Accessed July 11, 2014].

Price DL, Rockenstein E, Ubhi K, Phung V, MacLean-Lewis N, Askay D, Cartier A, Spencer B, Patrick C, Desplats P, Ellisman MH, Masliah E (2010) Alterations in mGluR5 Expression and Signaling in Lewy Body Disease and in Transgenic Models of Alpha-Synucleinopathy - Implications for Excitotoxicity Okazawa H, ed. PLoS One 5:e14020.

Purgert CA, Izumi Y, Jong Y-JI, Kumar V, Zorumski CF, O’Malley KL (2014) Intracellular mGluR5 Can Mediate Synaptic Plasticity in the Hippocampus. J Neurosci 34:4589-4598.

Qi C, Xu M, Gan J, Yang X, Wu N, Song L, Yuan W, Liu Z (2014) Erythropoietin improves neurobehavior by reducing dopaminergic neuron loss in a 6-hydroxydopamine-induced rat model. Int J Mol Med 34:440-450 Available at: http://www.ncbi.nlm.nih.gov/pubmed/24939444 [Accessed June 19, 2014].

Qin Z, Zhu H, Hu Y (2009) Effects of lysophosphatidylcholine on beta-amyloid-induced neuronal apoptosis. Acta Pharmacol Sin 
$30: 388-395$.

Quinn MT, Parthasarathy S, Steinberg D (1988)

Lysophosphatidylcholine: a chemotactic factor for human

monocytes and its potential role in atherogenesis. Proc Natl Acad

Sci U S A 85:2805-2809.

Quiroz JA, Tamburri P, Deptula D, Banken L, Beyer U, Rabbia M, Parkar N, Fontoura P, Santarelli L (2016) Efficacy and Safety of Basimglurant as Adjunctive Therapy for Major Depression.

JAMA Psychiatry 73:675 Available at:

http://archpsyc.jamanetwork.com/article.aspx?doi=10.1001/jamap sychiatry.2016.0838.

Ravnsjkjaer K, Frigerio F, Boegesen M, Nielsen T, Maechler P, Mandrup S (2014) PPAR-gamma is a fatty acid sensor that enhances mitochondrial oxidation in insulin-secreting cells and protects against fatty acid-induced dysfunction. J lipid Res $51: 1370-1379$.

Reilmann R, Rouzade-Dominguez M-L, Saft C, Süssmuth SD, Priller J, Rosser A, Rickards H, Schöls L, Pezous N, Gasparini F, Johns D, Landwehrmeyer GB, Gomez-Mancilla B (2015) A randomized, placebo-controlled trial of AFQ056 for the treatment of chorea in Huntington's disease. Mov Disord 30:427-431 Available at: http://www.ncbi.nlm.nih.gov/pubmed/25689146.

Rial Verde EM, Lee-Osbourne J, Worley PF, Malinow R, Cline HT 
(2006) Increased expression of the immediate-early gene arc/arg3.1 reduces AMPA receptor-mediated synaptic transmission. Neuron 52:461-474 Available at: http://www.ncbi.nlm.nih.gov/pubmed/17088212.

Ribeiro FM, Vieira LB, Pires RGW, Olmo RP, Ferguson SSG (2017) Metabotropic glutamate receptors and neurodegenerative diseases. Pharmacol Res 115:179-191.

Robinson S, Krentz L, Moore C, Meshul CK (2001) Blockade of NMDA receptors by MK-801 reverses the changes in striatal glutamate immunolabeling in 6-OHDA-lesioned rats. Synapse 42:54-61 Available at: http://doi.wiley.com/10.1002/syn.1099.

Rodriguez-Pallares J, Parga JA, Muñoz A, Rey P, Guerra MJ, Labandeira-Garcia JL (2007) Mechanism of 6-hydroxydopamine neurotoxicity: the role of NADPH oxidase and microglial activation in 6-hydroxydopamine-induced degeneration of dopaminergic neurons. J Neurochem 103:145-156 Available at: http://www.ncbi.nlm.nih.gov/pubmed/17573824.

Rodriguez AL (2005) A close structural analog of MPEP acts as a neutral allosteric site ligand on metabotropic glutamate receptor subtype 5 and blocks the effects of multiple allosteric modulators. Mol Pharmacol Available at: http://molpharm.aspetjournals.org/cgi/doi/10.1124/mol.105.01613 9. 
Roskoski R (2012) ERK1/2 MAP kinases: Structure, function, and regulation. Pharmacol Res 66:105-143 Available at: http://linkinghub.elsevier.com/retrieve/pii/S1043661812000977.

Rosner M, Siegel N, Valli A, Fuchs C, Hengstschläger M (2010) mTOR phosphorylated at S2448 binds to raptor and rictor. Amino Acids 38:223-228 Available at: http://www.ncbi.nlm.nih.gov/pubmed/19145465.

Rothblat DS, Schneider JS (1994) Spontaneous functional recovery from parkinsonism is not due to reinnervation of the dorsal striatum by residual dopaminergic neurons. Brain Res Bull 34:309-312 Available at: http://www.ncbi.nlm.nih.gov/pubmed/7914468 [Accessed November 27, 2017].

Roux PP, Topisirovic I (2012) Regulation of mRNA Translation by Signaling Pathways. Cold Spring Harb Perspect Biol 4:a012252a012252 Available at: http://cshperspectives.cshlp.org/lookup/doi/10.1101/cshperspect.a 012252.

Rudyk CA, McNeill J, Prowse N, Dwyer Z, Farmer K, Litteljohn D, Caldwell W, Hayley S (2017) Age and chronicity of administration dramatically influenced the impact of low dose paraquat exposure on behavior and hypothalamic-pituitaryadrenal activity. Front Aging Neurosci 9. 
Ryborg a. K, Deleuran B, Thestrup-Pedersen K, Kragballe K (1994) Lysophosphatidylcholine: a chemoattractant to human T lymphocytes. Arch Dermatol Res 286:462-465.

Rylander D, Iderberg H, Li Q, Dekundy A, Zhang J, Li H, Baishen R, Danysz W, Bezard E, Cenci MA (2010) A mGluR5 antagonist under clinical development improves L-DOPA-induced dyskinesia in parkinsonian rats and monkeys. Neurobiol Dis 39:352-361 Available at: http://linkinghub.elsevier.com/retrieve/pii/S096999611000152X.

Rylander D, Recchia A, Mela F, Dekundy A, Danysz W, Cenci MA (2009) Pharmacological Modulation of Glutamate Transmission in a Rat Model of L-DOPA-Induced Dyskinesia: Effects on Motor Behavior and Striatal Nuclear Signaling. J Pharmacol Exp Ther 330:227-235 Available at: http://jpet.aspetjournals.org/cgi/doi/10.1124/jpet.108.150425.

Sachs C, Jonsson G (1975) Mechanisms of action of 6hydroxydopamine. Biochem Pharmacol 24:1-8 Available at: http://linkinghub.elsevier.com/retrieve/pii/0006295275903044.

Salehi Z, Mashayekhi F (2009) Brain-derived neurotrophic factor concentrations in the cerebrospinal fluid of patients with Parkinson's disease. J Clin Neurosci 16:90-93.

Salmaso N, Silbereis J, Komitova M, Mitchell P, Chapman K, Ment LR, Schwartz ML, Vaccarino FM (2012) Environmental 
enrichment increases the GFAP+ stem cell pool and reverses hypoxia-induced cognitive deficits in juvenile mice. J Neurosci 32:8930-8939.

Sarbassov DD, Ali SM, Sengupta S, Sheen J-H, Hsu PP, Bagley AF, Markhard AL, Sabatini DM (2006) Prolonged rapamycin treatment inhibits mTORC2 assembly and Akt/PKB. Mol Cell 22:159-168 Available at: http://www.ncbi.nlm.nih.gov/pubmed/16603397.

Sargin D, Friedrichs H, El-Kordi A, Ehrenreich H (2010) Erythropoietin as neuroprotective and neuroregenerative treatment strategy: comprehensive overview of 12 years of preclinical and clinical research. Best Pract Res Clin Anaesthesiol 24:573-594 Available at: http://www.ncbi.nlm.nih.gov/pubmed/21619868 [Accessed November 14, 2013].

Sasi M, Vignoli B, Canossa M, Blum R (2017) Neurobiology of local and intercellular BDNF signaling. Pflugers Arch 469:593-610 Available at: http://www.ncbi.nlm.nih.gov/pubmed/28280960.

Sauer H, Oertel WH (1994) Progressive degeneration of nigrostriatal dopamine neurons following intrastriatal terminal lesions with 6hydroxydopamine: a combined retrograde tracing and immunocytochemical study in the rat. Neuroscience 59:401-415 Available at: http://www.ncbi.nlm.nih.gov/pubmed/7516500. 
Schäbitz W-R, Krüger C, Pitzer C, Weber D, Laage R, Gassler N, Aronowski J, Mier W, Kirsch F, Dittgen T, Bach A, Sommer C, Schneider A (2008) A neuroprotective function for the hematopoietic protein granulocyte-macrophage colony stimulating factor (GM-CSF). J Cereb Blood Flow Metab 28:2943 Available at: http://www.ncbi.nlm.nih.gov/pubmed/17457367 [Accessed November 26, 2013].

Scharf SH, Jaeschke G, Wettstein JG, Lindemann L (2015) Metabotropic glutamate receptor 5 as drug target for Fragile X syndrome. Curr Opin Pharmacol 20:124-134 Available at: http://linkinghub.elsevier.com/retrieve/pii/S1471489214001581.

Schober A (2004) Classic toxin-induced animal models of Parkinson's disease: 6-OHDA and MPTP. Cell Tissue Res 318:215-224 Available at: http://www.ncbi.nlm.nih.gov/pubmed/15503155.

Schwab RS, Doshay LJ, Garland H, Bradshaw P, Garvey E, Crawford B (1956) Shift to Older Age Distribution in Parkinsonism: A Report on 1,000 Patients Covering the Past Decade from Three Centers. Neurology 6:783-783 Available at: http://www.neurology.org/cgi/doi/10.1212/WNL.6.11.783.

Schweitzer KJ, Brüssel T, Leitner P, Krüger R, Bauer P, Woitalla D, Tomiuk J, Gasser T, Berg D (2007) Transcranial ultrasound in different monogenetic subtypes of Parkinson's disease. J Neurol 254:613-616 Available at: http://www.ncbi.nlm.nih.gov/pubmed/17415511 [Accessed July 329 
$11,2014]$.

Segura-Aguilar J, Kostrzewa RM (2015) Neurotoxin Mechanisms and Processes Relevant to Parkinson's Disease: An Update. Neurotox Res Available at: http://www.ncbi.nlm.nih.gov/pubmed/25631236.

Shen RY, Altar CA, Chiodo LA (1994) Brain-derived neurotrophic factor increases the electrical activity of pars compacta dopamine neurons in vivo. Proc Natl Acad Sci U S A 91:8920-8924 Available at: http://www.ncbi.nlm.nih.gov/pubmed/8090745.

Sherer T, Betarbet R, Greenamyre J (2001) Pathogenesis of Parkinson's disease. Curr Opin Investig drugs 2:654-662.

Sherer TB, Kim JH, Betarbet R, Greenamyre JT (2003) Subcutaneous rotenone exposure causes highly selective dopaminergic degeneration and alpha-synuclein aggregation. Exp Neurol 179:9-16 Available at:

http://www.ncbi.nlm.nih.gov/pubmed/12504863.

Shults CW, Kimber T, Altar CA (1995) BDNF attenuates the effects of intrastriatal injection of 6-hydroxydopamine. Neuroreport 6:1109-1112 Available at: http://www.ncbi.nlm.nih.gov/pubmed/7662888.

Sofic E, Riederer P, Heinsen H, Beckmann H, Reynolds GP, Hebenstreit G, Youdim MB (1988) Increased iron (III) and total iron content in post mortem substantia nigra of parkinsonian 
brain. J Neural Transm 74:199-205 Available at:

http://www.ncbi.nlm.nih.gov/pubmed/3210014 [Accessed

December 4, 2017].

Soto-Otero R, Méndez-Álvarez E, Hermida-Ameijeiras Á, Muñoz-

Patiño AM, Labandeira-Garcia JL (2002) Autoxidation and

Neurotoxicity of 6-Hydroxydopamine in the Presence of Some

Antioxidants. J Neurochem 74:1605-1612 Available at:

http://doi.wiley.com/10.1046/j.1471-4159.2000.0741605.x.

Spillantini MG, Schmidt ML, Lee VM-Y, Trojanowski JQ, Jakes R, Goedert M (1997) Alpha-synuclein in Lewy bodies. Nature 388:839-840 Available at:

http://www.nature.com/doifinder/10.1038/42166.

Spooren WPJ., Gasparini F, Bergmann R, Kuhn R (2000) Effects of the prototypical mGlu5 receptor antagonist 2-methyl-6(phenylethynyl)-pyridine on rotarod, locomotor activity and rotational responses in unilateral 6-OHDA-lesioned rats. Eur J Pharmacol 406:403-410 Available at: http://linkinghub.elsevier.com/retrieve/pii/S001429990000697X.

Stanic D, Finkelstein DI, Bourke DW, Drago J, Horne MK (2003) Timecourse of striatal re-innervation following lesions of dopaminergic SNpc neurons of the rat. Eur J Neurosci 18:11751188 Available at: http://doi.wiley.com/10.1046/j.14609568.2003.02800.x [Accessed November 27, 2017]. 
Steece-Collier K, Chambers LK, Jaw-Tsai SS, Menniti FS, Greenamyre JT (2000) Antiparkinsonian actions of CP-101,606, an antagonist of NR2B subunit-containing N-methyl-d-aspartate receptors. Exp Neurol 163:239-243 Available at: http://www.ncbi.nlm.nih.gov/pubmed/10785463.

Stocchi F, Rascol O, Destee A, Hattori N, Hauser RA, Lang AE, Poewe W, Stacy M, Tolosa E, Gao H, Nagel J, Merschhemke M, Graf A, Kenney C, Trenkwalder C (2013) AFQ056 in Parkinson patients with levodopa-induced dyskinesia: 13-week, randomized, dose-finding study. Mov Disord 28:1838-1846.

Stott SRW, Barker R a (2013) Time course of dopamine neuron loss and glial response in the 6-OHDA striatal mouse model of Parkinson's disease. Eur J Neurosci 39:1-15 Available at: http://www.ncbi.nlm.nih.gov/pubmed/24372914 [Accessed January 6, 2014].

Sulzer D (2007) Multiple hit hypotheses for dopamine neuron loss in Parkinson's disease. Trends Neurosci 30:244-250.

Sun X-R, Chen L, Chen W-F, Xue Y, Yung W-H (2013) Electrophysiological and behavioral effects of group III metabotropic glutamate receptors on pallidal neurons in normal and parkinsonian rats. Synapse 67:831-838 Available at: http://doi.wiley.com/10.1002/syn.21694.

Switon K, Kotulska K, Janusz-Kaminska A, Zmorzynska J, Jaworski J 
(2016) Molecular neurobiology of mTOR. Neuroscience 341:112-153.

Tagliaferro P, Burke RE (2016) Retrograde Axonal Degeneration in Parkinson Disease. J Parkinsons Dis 6:1-15 Available at: http://www.medra.org/servlet/aliasResolver?alias=iospress\&doi= 10.3233/JPD-150769.

Tanik SA, Schultheiss CE, Volpicelli-Daley LA, Brunden KR, Lee VMY (2013) Lewy body-like $\alpha$-synuclein aggregates resist degradation and impair macroautophagy. J Biol Chem 288:15194-15210 Available at:

http://www.ncbi.nlm.nih.gov/pubmed/23532841.

Tazangi PE, Moosavi SMS, Shabani M, Haghani M (2014) Erythropoietin improves synaptic plasticity and memory deficits by decrease of the neurotransmitter release probability in the rat model of Alzheimer's disease. Pharmacol Biochem Behav Available at: http://www.ncbi.nlm.nih.gov/pubmed/25553822 [Accessed January 8, 2015].

Testa CM, Standaert DG, Landwehrmeyer GB, Penney JB, Young AB (1995) Differential expression of mGluR5 metabotropic glutamate receptor mRNA by rat striatal neurons. J Comp Neurol 354:241-252.

Testa CM, Standaert DG, Young AB, Penney JB (1994) Metabotropic glutamate receptor mRNA expression in the basal ganglia of the 
rat. J Neurosci 14:3005-3018 Available at:

http://www.ncbi.nlm.nih.gov/pubmed/8182455.

Thiele SL, Warre R, Nash JE (2012) Development of a Unilaterallylesioned 6-OHDA Mouse Model of Parkinson's Disease. J Vis Exp.

Thomas B, Beal MF (2007) Parkinson's disease. Hum Mol Genet 16:R183-R194 Available at:

https://academic.oup.com/hmg/articlelookup/doi/10.1093/hmg/ddm159.

Thomas GM, Huganir RL (2004) MAPK cascade signalling and synaptic plasticity. Nat Rev Neurosci 5:173-183 Available at: http://www.ncbi.nlm.nih.gov/pubmed/14976517.

Tieu K (2011) A Guide to Neurotoxic Animal Models of Parkinson's Disease. Cold Spring Harb Perspect Med 1:a009316-a009316 Available at:

http://perspectivesinmedicine.cshlp.org/lookup/doi/10.1101/cshpe rspect.a009316.

Tison F, Keywood C, Wakefield M, Durif F, Corvol J-C, Eggert K, Lew M, Isaacson S, Bezard E, Poli S-M, Goetz CG, Trenkwalder C, Rascol O (2016) A Phase 2A Trial of the Novel mGluR5Negative Allosteric Modulator Dipraglurant for LevodopaInduced Dyskinesia in Parkinson's Disease. Mov Disord 00:1-8.

Toulouse A, Sullivan AM (2008) Progress in Parkinson's disease334 
Where do we stand? Prog Neurobiol 85:376-392 Available at: http://linkinghub.elsevier.com/retrieve/pii/S0301008208000567.

Treede I, Braun A, Sparla R, Kühnel M, Giese T, Turner JR, Anes E, Kulaksiz H, Füllekrug J, Stremmel W, Griffiths G, Ehehalt R (2007) Anti-inflammatory effects of phosphatidylcholine. J Biol Chem 282:27155-27164.

Trenkwalder C, Stocchi F, Poewe W, Dronamraju N, Kenney C, Shah A, von Raison F, Graf A (2016) Mavoglurant in Parkinson's patients with $<$ scp $>1</$ scp $>$-Dopa-induced dyskinesias: Two randomized phase 2 studies. Mov Disord 31:1054-1058.

Tsukahara T, Takeda M, Shimohama S, Ohara O, Hashimoto N (1995) Effects of brain-derived neurotrophic factor on 1-methyl-4phenyl-1,2,3,6-tetrahydropyridine-induced parkinsonism in monkeys. Neurosurgery 37:733-9; discussion 739-41 Available at: http://www.ncbi.nlm.nih.gov/pubmed/8559303.

Ungerstedt U (1968) 6-Hydroxy-dopamine induced degeneration of central monoamine neurons. Eur J Pharmacol 5:107-110 Available at: http://www.ncbi.nlm.nih.gov/pubmed/5718510.

Ungerstedt U (1971) Adipsia and aphagia after 6-hydroxydopamine induced degeneration of the nigro-striatal dopamine system. Acta Physiol Scand Suppl 367:95-122 Available at: http://www.ncbi.nlm.nih.gov/pubmed/4332694.

van der Putten H, Wiederhold KH, Probst a, Barbieri S, Mistl C, 335 
Danner S, Kauffmann S, Hofele K, Spooren WP, Ruegg M a, Lin S, Caroni P, Sommer B, Tolnay M, Bilbe G (2000)

Neuropathology in mice expressing human alpha-synuclein. J

Neurosci 20:6021-6029 Available at:

http://www.ncbi.nlm.nih.gov/pubmed/10934251.

Vernon AC, Zbarsky V, Datla KP, Croucher MJ, Dexter DT (2007)

Subtype selective antagonism of substantia nigra pars compacta

Group I metabotropic glutamate receptors protects the nigrostriatal system against 6-hydroxydopamine toxicity in vivo. J Neurochem 103:1075-1091 Available at: http://doi.wiley.com/10.1111/j.1471-4159.2007.04860.x.

Vest RS, O’Leary H, Coultrap SJ, Kindy MS, Bayer KU (2010) Effective post-insult neuroprotection by a novel $\mathrm{Ca}(2+) /$ calmodulin-dependent protein kinase II (CaMKII) inhibitor. J Biol Chem 285:20675-20682 Available at: http://www.ncbi.nlm.nih.gov/pubmed/20424167.

Villeneuve LM, Purnell PR, Boska MD, Fox HS (2016) Early Expression of Parkinson's Disease-Related Mitochondrial Abnormalities in PINK1 Knockout Rats. Mol Neurobiol 53:171186 Available at: http://www.ncbi.nlm.nih.gov/pubmed/25421206.

Vlajinac H, Dzoljic E, Maksimovic J, Marinkovic J, Sipetic S, Kostic V (2013) Infections as a risk factor for Parkinson's disease: a case-control study. Int J Neurosci 123:329-332 Available at: 
http://www.ncbi.nlm.nih.gov/pubmed/23270425.

Volk LJ, Daly CA, Huber KM (2006) Differential Roles for Group 1 mGluR Subtypes in Induction and Expression of Chemically Induced Hippocampal Long-Term Depression. J Neurophysiol 95:2427-2438 Available at: http://www.physiology.org/doi/10.1152/jn.00383.2005.

Volpicelli-Daley LA, Luk KC, Patel TP, Tanik SA, Riddle DM, Stieber A, Meaney DF, Trojanowski JQ, Lee VM-Y (2011) Exogenous $\alpha$-Synuclein Fibrils Induce Lewy Body Pathology Leading to Synaptic Dysfunction and Neuron Death. Neuron 72:57-71 Available at: http://linkinghub.elsevier.com/retrieve/pii/S0896627311008440.

Vranesic I, Ofner S, Flor PJ, Bilbe G, Bouhelal R, Enz A, Desrayaud S, McAllister K, Kuhn R, Gasparini F (2014) AFQ056/mavoglurant, a novel clinically effective mGluR5 antagonist: Identification, SAR and pharmacological characterization. Bioorg Med Chem 22:5790-5803 Available at: http://linkinghub.elsevier.com/retrieve/pii/S0968089614006798.

Wachtel H, Kunow M, Löschmann PA (1992) NBQX (6-nitrosulfamoyl-benzo-quinoxaline-dione) and CPP (3-carboxypiperazin-propyl phosphonic acid) potentiate dopamine agonist induced rotations in substantia nigra lesioned rats. Neurosci Lett 142:179-182 Available at: http://www.ncbi.nlm.nih.gov/pubmed/1280793. 
Wadley AJ, Veldhuijzen van Zanten JJCS, Aldred S (2013) The interactions of oxidative stress and inflammation with vascular dysfunction in ageing: the vascular health triad. Age (Omaha) 35:705-718 Available at: http://link.springer.com/10.1007/s11357-012-9402-1.

Wakabayashi K, Tanji K, Mori F, Takahashi H (2007) The Lewy body in Parkinson's disease: Molecules implicated in the formation and degradation of $\alpha$-synuclein aggregates. Neuropathology 27:494506 Available at: http://doi.wiley.com/10.1111/j.14401789.2007.00803.x.

Walsh S, Finn DP, Dowd E (2011) Time-course of nigrostriatal neurodegeneration and neuroinflammation in the 6hydroxydopamine-induced axonal and terminal lesion models of Parkinson's disease in the rat. Neuroscience 175:251-261 Available at: http://www.ncbi.nlm.nih.gov/pubmed/21145947 [Accessed November 5, 2013].

Wang H, Zhang Q, Wen Q, Zheng Y, Lazarovici P, Philip L, Jiang H, Lin J, Zheng W (2012) Proline-rich Akt substrate of 40kDa (PRAS40): a novel downstream target of PI3k/Akt signaling pathway. Cell Signal 24:17-24 Available at: http://www.ncbi.nlm.nih.gov/pubmed/21906675.

Williams LR (1991) Hypophagia is induced by intracerebroventricular administration of nerve growth factor. Exp Neurol 113:31-37 Available at: http://www.ncbi.nlm.nih.gov/pubmed/2044677. 
Winkler J, Ramirez GA, Kuhn HG, Peterson DA, Day-Lollini PA, Stewart GR, Tuszynski MH, Gage FH, Thal LJ (1997) Reversible Schwann cell hyperplasia and sprouting of sensory and sympathetic neurites after intraventricular administration of nerve growth factor. Ann Neurol 41:82-93.

Winklhofer KF, Haass C (2010) Mitochondrial dysfunction in Parkinson's disease. Biochim Biophys Acta 1802:29-44 Available at: http://www.ncbi.nlm.nih.gov/pubmed/19733240 [Accessed July 11, 2014].

Wright MM, Howe AG, Zaremberg V (2004) Cell membranes and apoptosis : role of cardiolipin , phosphatidylcholine , and anticancer lipid. Biochem Cell Biol 26:18-26.

Wright MM, McMaster CR (2002) Phospholipid synthesis, diacylglycerol compartmentation, and apoptosis. Biol Res $35: 223-229$.

Xue Y-QQ, Zhao L-RR, Guo W-PP, Duan W-MM (2007) Intrastriatal administration of erythropoietin protects dopaminergic neurons and improves neurobehavioral outcome in a rat model of Parkinson's disease. Neuroscience 146:1245-1258 Available at: http://www.ncbi.nlm.nih.gov/pubmed/17363174 [Accessed November 12, 2013].

Yahr MD, Duvoisin RC, Schear MJ, Barrett RE, Hoehn MM (1969) Treatment of Parkinsonism With Levodopa. Arch Neurol 21:343- 
354 Available at:

http://archneur.jamanetwork.com/article.aspx?articleid=569217.

Yamaguchi T, Wang HL, Morales M (2013) Glutamate neurons in the substantia nigra compacta and retrorubral field. Eur J Neurosci $38: 3602-3610$.

Yamasaki T, Fujinaga M, Kawamura K, Furutsuka K, Nengaki N, Shimoda Y, Shiomi S, Takei M, Hashimoto H, Yui J, Wakizaka H, Hatori A, Xie L, Kumata K, Zhang M-R (2016) Dynamic Changes in Striatal mGluR1 But Not mGluR5 during Pathological Progression of Parkinson's Disease in Human Alpha-Synuclein A53T Transgenic Rats: A Multi-PET Imaging Study. J Neurosci 36:375-384 Available at:

http://www.jneurosci.org/cgi/doi/10.1523/JNEUROSCI.228915.2016.

Yang L, Miao L, Liang F, Huang H, Teng X, Li S, Nuriddinov J, Selzer ME, Hu Y (2014) The mTORC1 effectors S6K1 and 4EBP play different roles in CNS axon regeneration. Nat Commun 5:5416 Available at:

http://www.nature.com/doifinder/10.1038/ncomms6416.

Zhang Y, Granholm A-C, Huh K, Shan L, Diaz-Ruiz O, Malik N, Olson L, Hoffer BJ, Lupica CR, Hoffman AF, Backman CM (2012) PTEN deletion enhances survival, neurite outgrowth and function of dopamine neuron grafts to MitoPark mice. Brain 135:2736-2749 Available at: 
https://academic.oup.com/brain/article-

lookup/doi/10.1093/brain/aws196.

Zheng B et al. (2010) PGC-1 $\alpha$, a potential therapeutic target for early intervention in Parkinson's disease. Sci Transl Med 2:52ra73

Available at:

http://www.pubmedcentral.nih.gov/articlerender.fcgi?artid=31299

86\&tool=pmcentrez\&rendertype $=$ abstract [Accessed July 11, 2014]. 